\title{
Health benefits and social determinants of the Mediterranean diet at a time of economic crisis : results from the moli-sani study
}

Citation for published version (APA):

Bonaccio, M. (2015). Health benefits and social determinants of the Mediterranean diet at a time of economic crisis : results from the moli-sani study. [Doctoral Thesis, Maastricht University]. Maastricht University. https://doi.org/10.26481/dis.20150318mb

Document status and date:

Published: 01/01/2015

DOI:

10.26481/dis.20150318mb

Document Version:

Publisher's PDF, also known as Version of record

Please check the document version of this publication:

- A submitted manuscript is the version of the article upon submission and before peer-review. There can be important differences between the submitted version and the official published version of record.

People interested in the research are advised to contact the author for the final version of the publication, or visit the DOI to the publisher's website.

- The final author version and the galley proof are versions of the publication after peer review.

- The final published version features the final layout of the paper including the volume, issue and page numbers.

Link to publication

\footnotetext{
General rights rights.

- You may freely distribute the URL identifying the publication in the public portal. please follow below link for the End User Agreement:

www.umlib.nl/taverne-license

Take down policy

If you believe that this document breaches copyright please contact us at:

repository@maastrichtuniversity.nl

providing details and we will investigate your claim.
}

Copyright and moral rights for the publications made accessible in the public portal are retained by the authors and/or other copyright owners and it is a condition of accessing publications that users recognise and abide by the legal requirements associated with these

- Users may download and print one copy of any publication from the public portal for the purpose of private study or research.

- You may not further distribute the material or use it for any profit-making activity or commercial gain

If the publication is distributed under the terms of Article 25fa of the Dutch Copyright Act, indicated by the "Taverne" license above, 
HEALTH BENEFITS AND SOCIAL DETERMINANTS OF THE MEDITERRANEAN DIET AT A TIME OF ECONOMIC CRISIS:

RESULTS FROM THE MOLI-SANI STUDY 
(C) Marialaura Bonaccio, Maastricht 2015

Thesis Maastricht University

ISBN: 978-88-8460-152-0

Layout: Marialaura Bonaccio

Cover: Oriano Cincindella

Printed by: Tipolitografia Fotolampo srl - Campobasso - Italy

All rights reserved. No part of this thesis may be reproduced, stored in a retrieval system or transmitted in any form or by any means, without the permission in writing from the author, or, when appropriate, of the publishers of the publications. 


\section{PROEFSCHRIFT}

ter verkrijging van de graad van doctor

aan de Universiteit Maastricht

op gezag van de Rector Magnificus,

Prof. dr. L.L.G. Soete

volgens het besluit van het College van Decanen,

in het openbaar te verdedigen op

Woensdag 18 Maart 2015 om 16.00 uur

door

Marialaura Bonaccio 


\section{Promotoren:}

Prof. dr. Hugo ten Cate

Prof. dr. Giovanni de Gaetano, Pozzilli, Italy

Prof. dr. Licia Iacoviello, Pozzilli, Italy

Beoordelings commissie:

Prof. dr. Harry Crijns (Chairman)

Prof. dr. Ilja Arts

Prof. dr. Salvatore Panico (Napoli, Italy)

Dr. Leon Schurgers

Prof. dr. Jos Vermylen (Leuven, Belgium)

The research described in this thesis was partially supported by the Italian Ministry of University and Research (MIUR- Programma Triennale di Ricerca, Decreto 1588), the Associazione Cuore Sano Onlus, Campobasso, Italy, and the Instrumentation Laboratory, Milan, Italy.

The printing of this thesis was generously provided by "Pasta La Molisana", Campobasso, Italy, in the frame of an initiative to support Young Molise Talents. 
To my family and friends and all thinkers 



\section{Chapter 1}

pag.

General Introduction

\section{Chapter 2}

The Mediterranean Diet: the reasons for a success

\section{Chapter 3}

Adherence to a Mediterranean diet is associated with a better health-related quality of life: a possible role of high dietary antioxidant content

\section{Chapter 4}

Adherence to the Mediterranean diet is associated with lower platelet and leukocyte counts: results from the Moli-sani study

\section{Chapter 5}

Adherence to the traditional Mediterranean diet and mortality in subjects with diabetes.

Prospective results from the Moli-sani study

\section{Chapter 6}

Low income is associated with poor adherence to a

Mediterranean diet and a higher prevalence of obesity:

cross-sectional results from the Moli-sani study

\section{Chapter 7}

Mass media information and adherence to Mediterranean diet: results from the Moli-sani study

\section{Chapter 8}

Nutrition knowledge is associated with higher adherence

to Mediterranean diet and lower prevalence of obesity.

Results from the Moli-sani study 
Decline of the Mediterranean diet at a time of economic crisis.

Results from the Moli-sani study

Chapter 10

Cardiovascular risk and events and country income stratum

Chapter 11

General Discussion

Chapter 12

Valorization

Chapter 13

General conclusions

Chapter 14

Summary

Chapter 15

Curriculum vitae

Chapter 16

Candidate's Publications

Chapter 17

Acknowledgements 
The traditional Mediterranean diet is an eating pattern typical of the Mediterranean basin. This dietary model was originally defined by the American scientist Ancel Keys who initially described the eating behaviors of the Mediterranean people living in Southern Italy; Keys was impressed by the observation that these people used to consume some specific foods more frequently compared to his fellows in US or those living in the Northern countries of Europe (1).

Likewise, he noted that Italian coronary heart disease death rates for middle-aged men in 1960 were less than half those for US men; moreover, Mediterranean population samples had lower levels of serum cholesterol, a major risk factor for atherosclerotic disease (2). These observations led him to establish a link between dietary behaviors and risk of coronary heart disease and his future investigations would have been mainly addressed to seek for evidence on this relationship.

The key-feature of the Mediterranean diet is a wide consumption of fruit and vegetables, non-refined grains, cereals, nuts, legumes, fish, olive oil as main fat source and moderate wine consumption preferably during main meals (3). Conversely, this pattern has low intake of dairy products and meat. Traditional lifestyle of Mediterranean people was also characterized by moderate to intense physical exercise mainly performed during the working hours that, in that time, were mostly of agricultural type.

Since Keys' first investigations, a large body of evidence has been linking the Mediterranean diet to reduced risk of major chronic diseases, such as cardiovascular and cerebrovascular disease, tumors and also neurodegenerative disease $(4,5)$.

Nevertheless, late evidence has been suggesting that Mediterranean societies are rapidly withdrawing from this eating pattern orienting their food choices toward products typical of the Western diet pattern, an unhealthy eating behavior characterized by high intake of refined grains, animal fats, sugars, processed meat but poor in legumes, cereals, fruits and vegetables.

The reasons people keep on shifting from healthy to unhealthy dietary habits remain open to several interpretations. Social changes appear to have consistently contributed to radical reversal in dietary habits in European Mediterranean societies even though developing countries are somewhat turning into westernized diets as well. Among possible causes, the increasing cost of many key-foods of the Mediterranean diet is likely to have led people to give up this eating pattern in favor of less expensive products which allow to save money but are definitively unhealthy (6). 
Previous evidence suggested that diet quality follows a socioeconomic gradient showing a linear relationship between food cost, eating patterns and obesity (7). Additionally, there is reason to believe that the economic downturn is going to produce unfavorable effects on the dietary lifestyle of people, especially among the less wealthy groups.

This thesis has a double purpose:

1. Firstly, it will examine whether the Mediterranean diet does still provide any health benefits within different population groups.

2. Secondly, it will address the socioeconomic and cultural determinants of the adherence to this eating behavior by further accounting for any likely effect of the economic crisis.

\section{Addressing the beneficial effects of the Mediterranean diet in the XXI century} The review here presented (Chapter 2) provides a summary of the beneficial effects of the Mediterranean diet on different health outcomes. As argued in a recent commentary by Jeremiah Stamler, one of the pioneer researchers of the Mediterranean diet and trusty collaborator of Ancel Keys, several things have changed since the original definition of this eating pattern and many problematic nutritional aspects emerged (2). The high grain product intake almost exclusively from white flour (not whole grain flour), the high total energy and salt intake are just some examples of how modern dietary habits shifted from the traditional dietary pattern. Despite this, the Mediterranean diet of the XXI century is still able to provide health advantages as reportedly documented by numerous investigations.

In agreement with this hypothesis, the following three papers presented in this thesis (Chapters 3,4,5) offer an overview of the findings from the MOLI-SANI study that clearly establish a relationship between adherence to this pattern and health benefits on selected outcomes of interest.

MOLI-SANI is a population-based cohort study which randomly recruited about 25,000 citizens of Molise, a region placed between Central and Southern Italy with the main purpose of investigating the genetic and environmental risk factors in the onset of major chronic diseases.

An original paper using data from the MOLI-SANI study (Chapter 3) investigated the association of adherence to the Mediterranean diet with quality of life as measured by the 36-Item Short Form Health Survey questionnaire.

In addition, the work discussed the likely account of both dietary antioxidant and fiber content for the observed relationship. Together with other psychological or psychiatric disorders such as depression or anxiety, mental health is one of the lately 
featured topics on which scientific research has been questioning during the very last years due to its reported association with mortality and other health outcomes.

One of the most likely explanations for the benefits offered by the Mediterranean diet relies in the anti-inflammatory action of some of its key-foods. Fruit and vegetables, olive oil and red wine are plenty of antioxidant compounds which have been ascribed a pivotal role in lowering subclinical inflammation.

Chapter 4 presents the original findings regarding the adherence to a Mediterranean diet and the resulting lower platelet and white blood cell counts, two recognized markers of low-grade inflammation, a condition strictly linked to incidence of both cardiovascular and cancer events.

As well as in the previous work, in this paper too the role of some key-components of the Mediterranean pyramid in explaining the observed association is discussed in detail.

Since the favorable effect of the Mediterranean diet has been reported also in high risk groups, attention has been paid to the impact of the Mediterranean diet against mortality within a population sample of subjects with diabetes further addressing the contribution of single foods in the generation of the observed association (Chapter 5).

\section{Socioeconomic and cultural determinants of the adherence to a Mediterranean diet}

Chapter 6 introduces the second part of the thesis that is mainly focused on the socioeconomic and cultural determinants of adherence to the Mediterranean diet or conversely the shifting from it.

Here the socioeconomic gradient in adherence to the Mediterranean diet within the MOLI-SANI cohort is discussed. As well as the whole country, this Southern area of Italy is not characterized by huge socioeconomic gaps, at least not comparable to those reported in other European countries. Thus the purpose was to test whether socioeconomic inequities may emerge also in a pretty homogenous socioeconomic environment.

Disparities in material resources are not the only mechanism by which inequities occur and consolidate within the social tissue.

Chapters 7 and 8 report findings from a subsample of the MOLI-SANI study who underwent an additional investigation aimed to assess both the exposure to mass media information and the health-related knowledge with a specific focus on nutrition. Both studies integrate a recent interest of research which is making efforts to identify novel factors other than material resources to explain the health gradient across population groups (8).

Of notice, the impact of mass media information exposure was investigated per se, 
not just as a mere indicator of physical inactivity, on several parameters of cardiovascular risk with particular regard to dietary habits.

Nutrition knowledge and exposure to mass media represent two main behaviorconditioning agents and their understanding is likely to be crucial to define new and more effective public health policies for the prevention of major chronic disease.

There is reason to believe that social disparities in health may become more evident in a period of economic instability. By focusing on the quick changes occurred in the dietary habits of people in a very short period of time, data from the MOLI-SANI study were used to seek for an association between the fall of the adherence to the Mediterranean diet and of material/cultural resources, in two different periods of time, before (2005-2006) and after (2007-2010) the economic downturn became manifested (Chapter 9). Chapter 10 briefly comments the results of a recently published international study reporting that although the Interheart risk score poses high-income countries at greater cardiovascular risk, the rates of major events and death are substantially higher in low-income countries (9).

Moreover, this thesis aims at considering the latest indications showing alarming trends in the adherence to this pattern.

Indeed, recent investigations have highlighted a dramatic fall of the Mediterranean diet within Mediterranean societies which seem definitely oriented towards lifestyles more typical of the Western diet pattern (10). Data from Italy (11) and Spain (12) revealed a gradual drop out of this eating pattern that is poorly followed by the youngest groups but is losing ground also within the elderly individuals (11). At the same time, the widespread conversion to Westernized behaviors has been linked to increased rates of obesity once characterizing the Anglo-Saxon populations and now widely diffused also in the Mediterranean areas.

Data here included (Chapter 9) show the degree of adherence to the Mediterranean diet within the MOLI-SANI population. Noticeably, the number of subjects eating "mediterraneanly" rapidly decreased year after year; in 2005, more than $30 \%$ of individuals used to refer of a Mediterranean diet behavior whereas this percentage was surprisingly halved just 4 years later.

\section{LIST OF PAPERS INCLUDED IN THIS THESIS}

1.Bonaccio M, Iacoviello L, de Gaetano G, On Behalf Of The Moli-Sani Investigators. The Mediterranean diet: The reasons for a success. Thromb Res. 2012;129:401-4. (Chapter 2) 
2.Bonaccio M, Di Castelnuovo A, Bonanni A, Costanzo S, De Lucia F, Zito F, Pounis G, Donati MB, de Gaetano G, Iacoviello L. Adherence to a Mediterranean diet is associated with a better health-related quality of life: a possible role of high dietary antioxidant content. BMJ Open. 2013 Aug 13;3(8). (Chapter 3)

3.Bonaccio M, Di Castelnuovo A, De Curtis A, Costanzo S, Persichillo M, Donati $\mathrm{MB}$, Cerletti C, Iacoviello L and de Gaetano G. Adherence to the Mediterranean diet is associated with lower platelet and leukocyte counts: results from the Molisani study. Blood. 2014;123:3037-44. (Chapter 4)

4.Bonaccio M, Di Castelnuovo A, Costanzo S, Persichillo M, De Curtis A, Donati $\mathrm{MB}$, de Gaetano $\mathrm{G}$ and Iacoviello L. Adherence to the traditional Mediterranean diet and mortality in subjects with diabetes. Prospective results from the MOLISANI study. Eur J Prev Cardiol. 2015 Feb 3. pii: 2047487315569409. [Epub ahead of print] (Chapter 5)

5.Bonaccio M, Bonanni AE, Di Castelnuovo A, De Lucia F, Donati MB, de Gaetano $\mathrm{G}$ and Iacoviello L, on behalf of the Moli-sani Project Investigators. Low income is associated with poor adherence to a Mediterranean diet and a higher prevalence of obesity: cross-sectional results from the Moli-sani study. BMJ Open 2012;2:e001685. (Chapter 6)

6.Bonaccio M, Di Castelnuovo A, Costanzo S, De Lucia F, Olivieri M, Donati MB, de Gaetano G, Iacoviello L, Bonanni A. Mass media information and adherence to Mediterranean diet: results from the Moli-sani study. Int J Public Health. 2012;57:589-97. (Chapter 7)

7.Bonaccio M, Di Castelnuovo A, Costanzo S, De Lucia F, Olivieri M, Donati MB, de Gaetano G, Iacoviello L, Bonanni A. Nutrition knowledge is associated with higher adherence to Mediterranean diet and lower prevalence of obesity. Results from the Moli-sani study. Appetite. 2013;68:139-46. (Chapter 8)

8.Bonaccio M, Di Castelnuovo A, Bonanni A, Costanzo S, De Lucia F, Persichillo M, Zito F, Donati MB, de Gaetano G, Iacoviello L, on behalf of the Moli-sani project Investigators. Decline of the Mediterranean diet at a time of economic crisis. Results from the Moli-sani study. Nutrition Metabolism and Cardiovascular disease. 2014;24:853-60. (Chapter 9)

9.Bonaccio M, Di Castelnuovo A, Iacoviello L. Cardiovascular risk and events and country income stratum. N Engl J Med. 2015;372:288-9. (Chapter 10) 
1. Keys A. Seven countries. A multivariate analysis of death, coronary heart disease. Cambridge, MA: Harvard University Press, 1980.

2. Stamler J. Toward a modern Mediterranean diet for the 21st century. Nutr Metab Cardiovasc Dis. 2013;23:1159-62.

3. Willett WC, Sacks F, Trichopoulou A, Drescher G, Ferro-Luzzi A, Helsing E, Trichopoulos D. Mediterranean diet pyramid: a cultural model for healthy eating. Am J Clin Nutr. 1995;61(6 Suppl):1402S-1406S.

4. Trichopoulou A, Costacou T, Bamia C, Trichopoulos D. Adherence to a Mediterranean diet and survival in a Greek population. N Engl J Med. 2003;348:2599-608.

5. Sofi F, Abbate R, Gensini GF, Casini A. Accruing evidence on benefits of adherence to the Mediterranean diet on health: an updated systematic review and meta-analysis. Am J Clin Nutr. 2010;92:1189-96.

6. Lopez CN, Martinez-Gonzalez MA, Sanchez-Villegas A, Alonso A, Pimenta AM, Bes-Rastrollo M. Costs of Mediterranean and western dietary patterns in a Spanish cohort and their relationship with prospective weight change. J Epidemiol Community Health. 2009;63:920-7.

7. Drewnowski A. Obesity, diets, and social inequalities. Nutr Rev. 2009;67 Suppl 1:S36-9.

8. Skalická V, van Lenthe F, Bambra C, Krokstad S, Mackenbach J. Material, psychosocial, behavioural and biomedical factors in the explanation of relative socio-economic inequalities in mortality: evidence from the HUNT study. Int J Epidemiol. 2009;38:1272-84.

9. Yusuf S, Rangarajan S, Teo K et al. Cardiovascular risk and events in 17 low-, middle-, and high-income countries. N Engl J Med. 2014;371:818-27.

10.Grosso G, Marventano S, Giorgianni G, Raciti T, Galvano F, Mistretta A. Mediterranean diet adherence rates in Sicily, southern Italy. Public Health Nutr. 2014;17:2001-9.

11. di Giuseppe R, Bonanni A, Olivieri M, Di Castelnuovo A, Donati MB, de Gaetano G, Cerletti C, Iacoviello L. Adherence to Mediterranean diet and anthropometric and metabolic parameters in an observational study in the 'Alto Molise' region: the MOLI-SAL project. Nutr Metab Cardiovasc Dis. 2008;18:415-21.

12. León-Muñoz LM, Guallar-Castillón P, Graciani A, et al. Adherence to the Mediterranean diet pattern has declined in Spanish adults.J Nutr. 2012;142:184350 . 


\section{CHAPTER 2.}

\section{Mini Review}

The Mediterranean diet: The reasons for a success

Marialaura Bonaccio, Licia Iacoviello, Giovanni de Gaetano, on behalf of the Molisani Investigators

Thromb Res. $2012 ; 129: 401-4$. 
Mini Review

\title{
The Mediterranean diet: The reasons for a success
}

\author{
Marialaura Bonaccio, Licia Iacoviello, Giovanni de Gaetano *, on behalf of the Moli-sani Investigators ${ }^{1}$ \\ Laboratori di Ricerca, Fondazione di Ricerca e Cura "Giovanni Paolo II", Largo Gemelli, 1, 86100 Campobasso, Italy
}

\section{A R T I C L E I N F O}

\section{Article history:}

Received 20 September 2011

Received in revised form 20 September 2011

Accepted 18 October 2011

Available online 17 November 2011

\section{Keywords:}

Mediterranean diet

Western diet

Cardiovascular risk

Alcohol

Socio-economic status

Income

\begin{abstract}
A B S T R A C T
There is a substantial body of evidence linking Mediterranean Diet to cardiovascular risk reduction and prevention of the major chronic diseases. Nevertheless Mediterranean societies are rapidly withdrawing from this eating pattern orienting their food choices toward products typical of the Western diet pattern, which is rich in refined grains, animal fats, sugars, processed meat but are quite poor in legumes, cereals, fruits and vegetables. The reasons people keep on shifting from healthy to unhealthy dietary habits remain open to several interpretations. Social changes appear to have consistently contributed to radical reversal in dietary habits in European Mediterranean societies even though developing Countries are somewhat turning into westernized diets as well.

Among possible causes, increasing prices of some of the major food items of Mediterranean pyramid seem to have led people to give up this eating pattern in favor of less expensive products which allow to save money but are definitively unhealthy.

Many studies suggest that diet quality follows a socio-economic gradient highlighting how disadvantaged people present higher rates of obesity, diabetes, cardiovascular disease and some types of cancer. Recent studies have shown a linear relationship between food cost and adherence to eating patterns and obesity. In addition to financial crisis, during the last decades the Mediterranean Diet has been put on the spot because of its alcohol -in- moderation component. Does it make any sense to blame a whole philosophy, which turned out to have beneficial effects on human health, just because, in some Countries, there is a misuse of alcoholic beverages?
\end{abstract}

(c) 2011 Elsevier Ltd. All rights reserved.

\section{Contents}

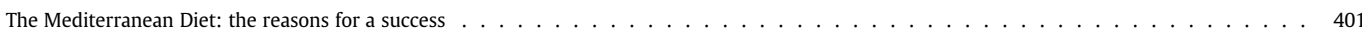

The Mediterranean paradox and the Finnish "miracle" . . . . . . . . . . . . . . . . . . . . . . . . . . . . . . . . . . . . . . . . . . . . . . . . .

Good for heart, good for mind . . . . . . . . . . . . . . . . . . . . . . . . . . . . . . . 402

Why do people keep Mediet at distance? $\quad \ldots \ldots \ldots$

Highest prices, lowest adherence . . . . . . . . . . . . . . . . . . . . . . . . . . . . . 403

The controversy on alcohol: an attack to the heart of Mediet? . . . . . . . . . . . . . . . . . . . . . . . . . . 403

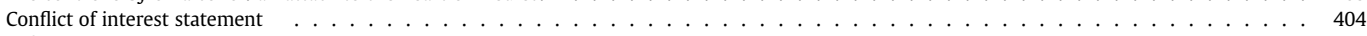

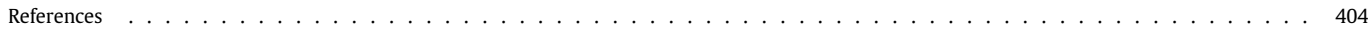

\section{The Mediterranean Diet: the reasons for a success}

"The Mediterranean diet is a set of traditional practices, knowledge and skills passed on from generation to generation and providing a sense of

Abbreviations: Mediet, Mediterranean Diet.

* Corresponding author at: Laboratori di Ricerca, Fondazione di Ricerca e Cura "Giovanni Paolo II", Largo Gemelli, 1, 86100 CAMPOBASSO, Italy. Tel.: + 390874312 280; fax: + 39 0874312710 .

E-mail address: gdegaetano@rm.unicatt.it (G. de Gaetano).

1 The list of the Moli-sani Investigators is reported in ref [30].

0049-3848/\$ - see front matter ( 2011 Elsevier Ltd. All rights reserved. doi:10.1016/j.thromres.2011.10.018 belonging and continuity to the concerned communities". This is the first reason cited by the UNESCO for recognizing the Mediterranean Diet (Mediet) as an Intangible Cultural Heritage [1]. This international organization has officially ratified what science has been continuously demonstrating during the last decades.

From what we read in the 5-point motivation, it appears quite clear that the UNESCO does not consider Mediet just as a mere collection of some selected foods but ascribes to the eating habits typical of the Mediterranean basin a cultural-promotion role deserving a special mention as plainly stated in the description of Mediet: "The Mediterranean diet encompasses more than just food. It promotes social interaction, since 
communal meals are the cornerstone of social customs and festive events. It has given rise to a considerable body of knowledge, songs, maxims, tales and legends. The system is rooted in respect for the territory and biodiversity, and ensures the conservation and development of traditional activities and crafts linked to fishing and farming in the Mediterranean communities of which Soria in Spain, Koroni in Greece, Cilento in Italy and Chefchaouen in Morocco are examples. Women play a particularly vital role in the transmission of expertise, as well as knowledge of rituals, traditional gestures and celebrations, and the safeguarding of techniques".

Living in a Mediterranean way means having healthy food but also adopting a specific way of life made of conviviality, traditions and daily moderate physical exercise. It is probably the reason for a success began 60 years ago when Ancel Keys thought to have found the elixir of life in the Cilento region, in Southern Italy.

Yet he would never have expected that one day the Mediterranean world would have decided to get rid of it.

Despite all, the intuition of the American physiologist turned out to be tremendous in learning that food has a great role in determining people's health status. His first investigations on the role of "bad" cholesterol on atherosclerosis and other cardiovascular diseases opened the way to a huge and prolific field of research corroborating what philosophers have always suspected. In some way, we really are what we eat. And our health is deeply linked to what we have for breakfast, lunch and dinner every single day.

More recently the torch passed to Antonia and Dimitri Trichopoulos, the Greek researchers who learnt very well the lesson from Keys' teachings. This searching couple started from Greece to carefully study the relationship between eating habits and health. Their first observations came from the wards of a hospital, similarly to what Keys did when he stayed in Naples with the US Army. Later on, one of their papers appeared in the New England Journal of Medicine establishing an impressive relationship between a standardized score to set the adherence to the traditional Mediet and the reduction of cardiovascular disease risk. The latter was accompanied by a comparable reduction of both cancer and total mortality [2].

Indeed science's interest in the effect of food on health has impressively grown in recent years as witnessed by the huge scientific production on this specific field of medicine.

\section{The Mediterranean paradox and the Finnish "miracle"}

The heart-saving diet is disappearing from the tables of the Countries in which it was born while is being adopted, at least in part, by populations not actually confident with it. What happened in Finland some decades ago is representative of the bizarre destiny of Mediet.

It was back in 1972, the name of the "mission" was the North Karelia Project [3]. Within 25 years the land of lakes has managed to completely change its face. The taskforce assigned to improve Finns' health by reducing the major risk factors did every effort to uproot the bad lifestyle of citizens. Smoking, of course, was the first major target to be bombed. If in the early 70's, 52 percent of middle-aged Finnish men used to smoke, in 1997 "only" 31 percent used to. The most surprising results came however from the eating habits changes.

Fruits and vegetables were unknown for the greatest part of the population, on the tables a slice of bread with butter was literally indefeasible for over 90 percent of Finns. Today, less than 7 percent of people have bread and butter, and their cholesterol levels decreased by an average of 17 percent. Blood pressure is constantly monitored and there has been a positive increase in the level of physical activity.

In the mid-'90s, the annual mortality from heart disease in middle-aged Finnish men (under 65) was reduced by 73 percent compared to the period 1967-71 and the most significant lowering of the rate of mortality occurred from the very start of the program, remaining constant until the mid-80s.
Over the last decade, the decline of cardiovascular mortality is set to eight percent a year. Since cardiovascular disease is the leading cause of death, total mortality also gained benefit, reducing by 45 percent and significantly expanding the life expectancy of Finns, seven years longer for men, six for women. The "restyling" of Finnish lifestyle in the wake of the Mediterranean lesson really gained good results, saving lives and nation's coffers

On the other side, while northern European Countries were appreciating the benefits of healthy living models, in Italy things were dramatically falling over. Two studies [4,5], conducted in Molise, a southern region of the Country, revealed a threatening scenario in the eating habits of people (Table 1 ). Despite being a region with a strong Mediterranean tradition, the percentage of people following Mediet in 2009 is far lower than the lowest of Nicotera and Pollica populations reported in the Sixties indicating a worrying change in the eating habits of Mediterranean areas.

\section{Good for heart, good for mind}

Lifestyle and eating habits are the major modifiable factors people can manage to keep at bay chronic disease. Increasing the levels of physical activity and introducing healthy food in everyday meals turned out to yield good results for health.

There is a substantial body of evidence linking Mediet to cardiovascular risk reduction and prevention of the major chronic diseases [6]. A recent meta-analysis by researchers in Florence took into account a large number of subjects and studies showing a significant and consistent protection provided by adherence to the Mediet in relation to the onset of major chronic degenerative diseases [7]. (Table 2)

More recently the team of EPICOR study has set a new score to evaluate adherence to Mediet in Italian population by using a list of foods typical of Italian meals and they did find that highest adherence was significantly linked to a reduction in the risk of stroke [8]. The score sounds as a sort of "Italian reply" to that elaborated by the Trichopoulos for the Greek population [2].

However both scores used an a priori approach: basically, they elaborated a method and then sought to see whether the observed eating habits could be encompassed in what they established before. Our team used a different approach to evaluate whether adherence could be somehow related to different cardiovascular profile and consequent cardiovascular risk reduction in a general Italian population by adopting an a posteriori technique [9]. We found that a Mediterranean diet-like aggregation of foods was positively associated with a significant reduction of cardiovascular risk intended also as lowering of some markers of inflammation, such as C-reactive protein. It is interesting to note that in the healthy cluster there were also some unhealthy foods suggesting that eating habits need to be considered as a whole rather than a mere list of foods.

Different studies have also compared Mediet to other diets, to see which could offer the best protection against the major noncommunicable chronic diseases.

Table 1

Score of adherence to Mediterranean Diet in the Moli-sani cohort in respect to similar scores measured in two previous studies (Nicotera and Pollica). For details, see ref 4 acores 5 and

\begin{tabular}{llll}
\hline & 25th percentile & 50th percentile & 75th percentile \\
\hline MEN & & & \\
NICOTERA 1960 & 5.4 & 7.5 & 10.8 \\
POLLICA 1967 & 3.2 & 6.0 & 8.4 \\
MOLI-SANI 2009 & $\mathbf{2 . 5}$ & $\mathbf{3 . 6}$ & $\mathbf{5 . 4}$ \\
WOMEN & & - & - \\
NICOTERA 1960 & - & 6.0 & 10.0 \\
POLLICA 1967 & 3.3 & $\mathbf{2 . 6}$ & $\mathbf{3 . 6}$ \\
MOLI-SANI 2009 & $\mathbf{2 . 0}$ & &
\end{tabular}


Table 2

Relative risks and $95 \%$ Confidence intervals of mortality from different causes or incidence of neurodegenerative diseases. Data derived from ref. 7 .

\begin{tabular}{ll}
\hline & RR 95\% CI \\
\hline Total mortality & $0.92(0.90-0.94)$ \\
Cardiovascular Mortality & $0.90(0.87-0.93)$ \\
Cancer Mortality & $0.94(0.92-0.96)$ \\
Neurodegenerative Disease Incidence & $0.87(0.81-0.94)$ \\
\hline
\end{tabular}

In a recent meta-analysis the Mediterranean eating pattern turned out to be more effective than low-fat diets in inducing important long-term changes in cardiovascular risk factors and inflammatory markers [10]. Further evidence comes from the study conducted by the Epicor team in Italy which showed that women in the highest quartile of vegetables and olive oil consumption resulted to have lower incidence of coronary heart disease compared to those in the lowest quartile [11].

As already noted by the Trichopoulos [2], it is not just the cardiovascular field to benefit from the Mediet which resulted to have relevant healthy effects also in relation to overall mortality, as lately confirmed by the EPIC investigation conducted in the Spanish cohort of the largest study on eating habits and tumors ever conducted in Europe [12]. According to researchers, olive oil-rich Mediet was related to a significant reduction in all-cause mortality and reduced risk of mortality from cardiovascular disease in over 40,000 participants.

Good news came also for mental wellness. In addition to many studies supporting the beneficial effects of Mediet on the incidence of neurodegenerative disease as Parkinson's and Alzheimers's diseases [7, 13], a group of Spanish researchers found that higher adherence to Mediet is positively associated with better health-related quality of life [14]. According to these Authors, the study is one of the few highlighting the effects of whole eating patterns on health since other studies have mainly focused on the effects on quality of life of single dietary components.

\section{Why do people keep Mediet at a distance?}

At first sight, there is no reason to follow other diets than the Mediterranean one. Nutrition experts, epidemiologists and researchers in general are strongly convinced that the Mediterranean paradigm is absolutely good for health, as reported by scientific literature.

Nevertheless Southern European Countries are rapidly withdrawing from this eating pattern orienting their food choices toward products typical of the Western diet pattern, which is rich in refined grains, animal fats, sugars, processed meat but are quite poor in legumes, cereals, fruits and vegetables. The reason people keep on shifting from healthy to unhealthy dietary habits remains open to several interpretations. Social changes appear to have consistently contributed to radical reversal in dietary habits in Mediterranean societies even though developing Countries are somewhat turning into westernized diets as well.

Increasing prices of some of the major food items of Mediet pyramid seem to have led people to give up this eating pattern in favor of less expensive products which allow to save money but are definitively unhealthy.

Many studies suggest that diet quality follows a socio-economic gradient highlighting how disadvantaged people present higher rates of obesity, diabetes, cardiovascular disease and some types of cancer.

A recent review by Darmon et al. [15] concluded that higher-quality diets are mainly consumed by better educated and more affluent people while lower socio-economic groups tend to have lower quality diets thus exposing them to higher risk to develop diet-related diseases. Similar conclusions were reached by other investigations [16], all concluding that low socio-economic status groups end with having poorer diets. Preliminary data from the Moli-sani Project [5] show that adherence to
Mediet-like patterns is strongly associated with socio-economic status, in particular lower adherence with lower-income, indicating that socio-economic status and in particular income currently represent a crucial issue for dietary choices of Italian people [17]

Socio-economic status has been proven to affect also the cardiovascular risk profile of youths as showed by the NANHES investigation [18] Independent of race/ethnicity, 6 to 17-year-olds from low-income families showed higher prevalence of obesity, central obesity, sedentary behavior and tobacco exposure.

\section{Highest prices, lowest adherence}

Recent studies have shown a linear relationship between food cost and adherence to eating patterns and obesity. According to results emerged from a cohort of Spanish university graduates, subjects reporting highest scores for the Western dietary pattern spent less money on their daily food costs compared to those having highest adherence to Mediterranean dietary pattern [19]. These Authors stress that the expensive cost of Mediet could represent an economic barrier to be considered when counseling people about following a healthy diet since cost may be a prohibitive factor.

Moreover some experts suggest that there is a strong linkage between energy density and energy cost. In other words, energydense foods composed of refined grains, added sugars, or fats may represent the lowest-cost option to the consumer, suggesting that the association between poverty and obesity may be partially mediated by the low cost of energy-dense foods and may be reinforced by the high palatability of sugar and fat [20]. The Seattle Obesity Study showed that diet cost significantly mediated the pathway between income and diet quality measures suggesting that the social gradient in diet quality finds solid explanation in diet cost [21].

Thus the main issue to be addressed seems to be not only to provide further evidence to the beneficial effects of Mediet on health but also to think through new strategies in order to create better conditions for people to return to the Mediterranean way of living. Greater attention should be paid to youth eating behaviors which are definitively worrying as often warned by the World Health Organization reports on this issue. Overweight and obesity rates among children are impressively growing especially in the Mediterranean basin countries, for example in Southern Italy where we found the highest proportion of overweight or obese children in Europe [22].

Increasing obesity rates due to poorer eating habits and the relative increased risk to be affected by chronic diseases in the years to come appears to be a business for everyone and not only for health experts. The return to Mediterranean way is a complex problem calling to action all the actors of the social life of Mediterranean Countries. Lowering the prices of some basic products such as fruits and vegetables and improving their availability in the most citizens-attended places should represent the first step to reclaim a healthy living model.

\section{The controversy on alcohol: an attack to the heart of Mediet?}

In the summer of 2009 an interesting study was published in the British Medical Journal with a nice catchy title: Anatomy of health effect of Mediterranean diet [23]. The purpose of the research was to rank the components of the dietary pyramid and to investigate their relative role in relation to overall mortality. In this way, the study brought to public attention that the principal component of the Mediet score as a predictor of lower mortality was moderate consumption of ethanol, followed by low consumption of meat and meat products, and high consumption of vegetables, fruits and nuts, olive oil, and legumes. In the meantime, another highly reputed journal came up with a strong critique against any kind of alcohol consumption, regardless of amounts and ways of drinking [24]. According to The Lancet, alcohol banning would heal all wounds of our society. Given 
this confused scientific scenario, what are common people supposed to do? Drinking in moderation or banning alcohol from their lives?

Maybe it would be useful not to isolate a single element from the whole. The Mediet is a whole of healthy and less healthy foods put together in a pyramid. This eating pattern allows to eat everything provided that people respect amounts and frequency of eating. Deleting or banning foods from the pyramid may yield a destabilizing effect on the healthy benefits of the Mediet. It is like we would not remind that, together with a healthy diet, people should be encouraged to practice some physical exercise. The Mediet indeed is not just a way of eating specific food typical of the Mediterranean basin. It's more a kind of philosophy teaching us what to have for meal, of course, but also how to drink - during meals and without exceeding - and why we have to practice exercise. Whether one would remove just one component of this ancient philosophy the whole mechanism might collapse. The equilibrium is the strength of Mediet as well as every single part of a car is useful to make it move on the road. Moderate consumption of alcohol is absolutely an untouchable component of the pyramid and has been proven to be effective not only in primary [25-27] but also in secondary cardiovascular risk prevention [28,29].

\section{Conflict of interest statement}

No conflict of interest to declare.

\section{References}

[1] http://www.unesco.org/culture/ich/en/RL/00394.

[2] Trichopoulou A, Costacou T, Bamia C, Trichopoulos D. Adherence to a Mediterranean diet and survival in a Greek population. N Engl J Med 2003;26(348):2599-608.

[3] Puska P. Successful prevention of non-communicable diseases: 25 year experiences with North Karelia Project in Finland. Public Health Med 2002;4:5-7.

[4] di Giuseppe R, Bonanni A, Olivieri M, Di Castelnuovo A, Donati MB, de Gaetano G, et al. Adherence to Mediterranean diet and anthropometric and metabolic parameters in an observational study in the 'Alto Molise' region: the MOLI-SAL project. Nutr Metab Cardiovasc Dis 2008:18:415-21.

[5] Iacoviello L, Bonanni A, Costanzo S, De Curtis A, Di Castelnuovo A, Olivieri M, et al. The Moli-sani Project, a randomized, prospective cohort study in the Molise region in Italy; design, rationale and objectives. Italian J Public Health 2007;4:110-8.

[6] Willett WC, Sacks F, Trichopoulou A, Drescher G, Ferro-Luzzi A, Helsing E, et al. Mediterranean diet pyramid: a cultural model for healthy eating. Am J Clin Nutr 1995;61(6 Suppl):1402S-6S.

[7] Sofi F, Abbate R, Gensini GF, Casini A. Accruing evidence on benefits of adherence to the Mediterranean diet on health: an updated systematic review and metaanalysis. Am J Clin Nutr 2010:92:1189-96.

[8] Agnoli C, Krogh V, Grioni S, Sieri S, Palli D, Masala G, et al. A priori-defined dietary patterns are associated with reduced risk of stroke in a large Italian cohort. J Nutr 2011:141:1552-8

[9] Centritto F, Iacoviello L, di Giuseppe R, De Curtis A, Costanzo S, Zito F, et al. Moli-sani Investigators. Dietary patterns, cardiovascular risk factors and C-reactive protein in a healthy Italian population. Nutr Metab Cardiovasc Dis 2009;19:697-706.
[10] Nordmann AJ, Suter-Zimmermann K, Bucher HC, Shai I, Tuttle KR, Estruch R, et al Meta-analysis comparing mediterranean to low-fat diets for modification of cardiovascular risk factors. Am J Med 2011;124:841-51 e2.

[11] Bendinelli B, Masala G, Saieva C, Salvini S, Calonico C, Sacerdote C, et al. Fruit, vegetables, and olive oil and risk of coronary heart disease in Italian women: the EPICOR Study. Am J Clin Nutr 2011:93:275-83.

[12] Buckland G, Agudo A, Travier N, María Huerta J, Cirera L, Tormo MJ, et al. Adherence to the Mediterranean diet reduces mortality in the Spanish cohort of the European to the Mediterranean diet reduces mortality in the Spanish cohort of the European
Prospective Investigation into Cancer and Nutrition (EPIC-Spain). Br J Nutr 2011;17:1-11.

[13] Sofi F, Cesari F, Abbate R, Gensini GF, Casini A. Adherence to Mediterranean diet and health status: meta-analysis. BMJ 2008;337:a1344, doi:10.1136/bmj.a1344.

[14] Henríquez Sánchez P, Ruano C, de Irala J, Ruiz-Canela M, Martínez-González MA Sánchez-Villegas A. Adherence to the Mediterranean diet and quality of life in the SUN Project. Eur J Clin Nutr 2011 Aug 17.

[15] Darmon N, Drewnowski A. Does social class predict diet quality? Am J Clin Nutr 2008;87:1107-17.

[16] Shahar D, Shai I, Vardi H, Shahar A, Fraser D. Diet and eating habits in high and low socioeconomic groups. Nutrition 2005;21:559-66.

[17] Bonaccio M, Bonanni A, Di Castelnuovo A, De Lucia F, Donati MB, de Gaetano C et al. Mediterranean diet in a time of crisis: lower income, lower adherence. Eur J Nutr 2011;50:489-98.

[18] Ali MK, Bullard KM, Beckles GL, Stevens MR, Barker L, Narayan KM, et al. Household Income and Cardiovascular Disease Risks in U.S. Children and Young Adults: Analyses from NHANES 1999-2008. Diabetes Care 2011;34:1998-2004.

[19] Lopez CN, Martinez-Gonzalez MA, Sanchez-Villegas A, Alonso A, Pimenta AM, BesRastrollo M. Costs of Mediterranean and western dietary patterns in a Spanish cohort and their relationship with prospective weight change. J Epidemiol Community Health 2009:63:920-7.

[20] Drewnowski A, Specter SE. Poverty and obesity: the role of energy density and energy costs. Am J Clin Nutr 2004;79:6-16.

[21] Aggarwal A, Monsivais P, Cook AJ, Drewnowski A. Does diet cost mediate the relation between socioeconomic position and diet quality? Eur J Clin Nutr 2011:65: $1059-66$

[22] Ahrens W, Bammann K, Siani A, Buchecker K, De Henauw S, Iacoviello L, et al. IDEFICS Consortium. The IDEFICS cohort: design, characteristics and participation in the baseline survey. Int J Obes (Lond) 2011 Apr;35(Suppl 1):S3-S15.

[23] Trichopoulou A, Bamia C, Trichopoulos D. Anatomy of health effects of Mediterranean diet: Greek EPIC prospective cohort study. BMJ 2009;23 338:b2337.

[24] Beaglehole R, Bonita R. Alcohol: a global health priority. Lancet Vol 2009;373: 2173-4.

[25] Di Castelnuovo A, Rotondo S, Iacoviello L, Donati MB, De Gaetano G. Meta-analysis of wine and beer consumption in relation to vascular risk. Circulation 2002:105: 2836-44.

[26] Di Castelnuovo A, Costanzo S, Bagnardi V, Donati MB, lacoviello L, de Gaetano C. Alcohol dosing and total mortality in men and women: an updated metaanalysis of 34 prospective studies. Arch Intern Med 2006;166:2437-45.

[27] Costanzo S, Di Castelnuovo A, Dontai MB, Iacoviello L, de Gaetano G. Wine, beer or spirit drinking in relation to fatal and non-fatal3 cardiovascular events: a meta-analysis. Eur J Epidemiol 2011, doi:10.1007/s10654-011-9631-0.

[28] Costanzo S, Di Castelnuovo A, Donati MB, Iacoviello L, de Gaetano G. Alcohol consumption and mortality in patients with cardiovascular disease: a meta-analysis. J Am Coll Cardiol 2010;55:1339-47.

[29] Costanzo S, Di Castelnuovo A, Donati MB, lacoviello L, de Gaetano G. Cardiovascular and overall mortality risk in relation to alcohol consumption in patients with cardiovascular disease. Circulation 2010;121:1951-9.

[30] di Giuseppe R, Arcari A, Serafini M, Di Castelnuovo A, Zito F, De Curtis A, et al. Total dietary antioxidant capacity and lung function in an Italian population: a favorable role in premenopausal/never smoker women. Eur J Clin Nutr 2011 Aug 31. 



\section{CHAPTER 3.}

Adherence to a Mediterranean diet is associated with a better health-related quality of life: a possible role of high dietary antioxidant content

Bonaccio M, Di Castelnuovo A, Bonanni A, Costanzo S, De Lucia F, Zito F, Pounis G, Donati MB, de Gaetano G, Iacoviello L, on behalf of the Moli-sani project Investigators

BMJ Open. 2013 Aug 13;3(8). 


\title{
$\mathrm{BMJ} \quad$ Adherence to a Mediterranean diet is OPen associated with a better health-related quality of life: a possible role of high dietary antioxidant content
}

\author{
Marialaura Bonaccio, ${ }^{1,2}$ Augusto Di Castelnuovo, ${ }^{1}$ Americo Bonanni, ${ }^{1,3}$ \\ Simona Costanzo, ${ }^{1}$ Francesca De Lucia, ${ }^{1}$ George Pounis, ${ }^{1}$ Francesco Zito, ${ }^{1}$ \\ Maria Benedetta Donati, ${ }^{2}$ Giovanni de Gaetano, ${ }^{2}$ Licia lacoviello, ${ }^{2,4}$ \\ on behalf of the Moli-sani project Investigators*
}

To cite: Bonaccio $\mathrm{M}, \mathrm{Di}$ Castelnuovo A, Bonanni A, et al. Adherence to a Mediterranean diet is associated with a better health-related quality of life: possible role of high dietary antioxidant content. BMJ Open 2013:3:e003003. doi:10.1136/bmjopen-2013003003

- Prepublication history and additional material for this paper is available online. To view these files please visit the journal online (http://dx.doi.org/10.1136/ bmjopen-2013-003003)

*The Moli-sani Project Investigators are listed in the online supplementary appendix.

Received 5 April 2013 Revised 20 June 2013 Accepted 2 July 2013

For numbered affiliations see end of article.

Correspondence to Dr Licia lacoviello; licia.iacoviello@moli-sani.org

\section{ABSTRACT}

Objectives: Mediterranean diet (MD) is associated with a reduced risk of major chronic disease. Healthrelated quality of life (HRQL) is a valid predictor of mortality. The aim of this study is to investigate the association between MD and $\mathrm{HRQL}$ and to examine the possible role of dietary antioxidants, fibre content and/or fatty acid components.

Design: Cross-sectional study on a sample of Italian participants enrolled in the Moli-sani Project, a population-based cohort study. Food intake was recorded by the Italian European prospective investigation into cancer and nutrition study food frequency questionnaire. Adherence to MD was appraised by a Greek Mediterranean diet score (MDS), an Italian Mediterranean diet index (IMI) and by principal component analysis (PCA). HRQL was assessed by the 36 -Item Short Form Health Survey.

Setting: Molise region, Italy.

Participants: 16937 participants of 24325 Italian citizens (age $\geq 35$ ).

Main outcomes: Dietary patterns and HRQL.

Results: Mental health was associated consistently and positively with MDS, IMI and an 'Olive oil and vegetable' pattern (PCA1), but negatively with an 'Eggs and sweets' pattern (PCA3). Physical health was associated positively with MDS and PCA1, but negatively with a 'Meat and pasta' pattern. Subjects with the highest MD adherence had $42 \%$ (MDS), $34 \%$ (IMI) or $59 \%$ (PCA1) statistically significant multivariable odds of being in the uppermost level of mental health, as compared with subjects in the lowest category. The associations disappeared after further adjustment for either total food antioxidant content or dietary fibre, while they were not modified by the inclusion of either monounsaturated or polyunsaturated fatty acids. Individuals in the highest PCA1 or PCA3 had significantly higher odds of being in the top level of physical health.

Conclusions: Adherence to an MD pattern is associated with better HRQL. The association is stronger with mental health than with physical health. Dietary total antioxidant and fibre content independently explain this relationship.

\section{ARTICLE SUMMARY}

Article focus

- Investigating the association between adherence to the Mediterranean diet and health-related quality of life.

- Examining the possible role of dietary antioxidants, fibre intake and/or fatty acid components in explaining the association.

Key messages

- Mental health is positively associated with a Mediterranean diet and inversely linked to 'Eggs and sweets' dietary pattern.

- The association is mainly accounted for not only by the total antioxidant content but also by the fibre dietary content.

- Fatty acid compounds do not explain the association between the Mediterranean diet and health-related quality of life.

Strengths and limitations of this study

- This study is apparently the first to provide a likely account of dietary antioxidants for the direct association between the Mediterranean diet and health-related quality of life.

- For the first time, this topic was addressed by using simultaneously two a priori Mediterranean scores and the a posteriori dietary patterns obtained by principal component analysis.

- The present study was cross-sectional and shares all the limitations of this study type; in particular, the inference of possible causality is unwarranted.

\section{INTRODUCTION}

Mediterranean diet (MD) is a healthy eating pattern associated with reduced risk for cardiovascular and neurodegenerative diseases and some types of cancer. ${ }^{1-3}$ This dietary pattern is characterised by the wide consumption of plant foods, cereals, legumes, fish and olive oil as the main source of fat 
and moderate red wine consumption. The biological mechanisms for chronic disease prevention associated with an MD pattern are high amounts of antioxidants, polyphenols and other compounds such as monounsaturated and polyunsaturated fatty acids. ${ }^{4-7}$

Health-related quality of life (HRQL) refers to an individual's subjective evaluation of his own health and wellbeing $^{8}$ and has rapidly become an important issue in modern times since Western societies are facing a process of increasing population ageing. Self-perceived health status is closely associated with cardiovascular disease (CVD) and all-cause mortality and many studies have found that self-health ratings are important predictors of mortality for persons with cardiovascular disease,,${ }^{9} 10$ middle-aged subjects ${ }^{11}$ and young adults. ${ }^{12}$ So far, few studies have investigated the relationship between MD and self-rated health in a general population.

Recent evidence suggests a positive correlation between adherence to MD and HRQL, ${ }^{13} 14$ not only in adults but also in adolescents. ${ }^{15}$ Further evidence has recently supported the relationship between MD and depression showing a potential protective role of MD against the onset of depressive symptoms. ${ }^{16} 17$

One of the most accredited hypotheses is that MD is positively associated with better overall health status and reduced risk of major chronic diseases because of its high content of different beneficial compounds, such as antioxidants, largely present in leafy vegetables, fruits, olive oil and red wine, monounsaturated (olive oil) or polyunsaturated fatty acids (mainly from fish and nuts ${ }^{5}$ ), dietary fibre $^{18} 19$ or low glycaemic index. ${ }^{20}$

Our study aimed to test the association of MD and other dietary patterns with mental and physical health to examine the possible role played by total food antioxidant content (FAC), dietary fibre and monounsaturated or polyunsaturated fatty acids.

\section{SUBJECTS AND METHODS \\ Study population}

The study population consisted of participants in the Moli-sani Project, a population-based cohort study which randomly recruited 24325 citizens of Molise, a region placed between central and southern Italy. Between March 2005 and April 2010, men and women aged $\geq 35$ years were randomly recruited from subjects included in the city-hall registries of Molise. ${ }^{21}$ Exclusion criteria were pregnancy, disturbances in understanding/ willing processes, ongoing poly traumas or coma and refusal to sign the informed consent form.

After exclusion of subjects reporting a personal history of cardiovascular disease (angina, myocardial infarction, heart failure, revascularisation procedures and stroke; $5.7 \%$ ), cancer $(3.1 \%)$ or of those for whom there was no information available on health-related quality of life $(21.7 \%)$ or dietary habits $(3.9 \%), 16937$ subjects were finally included in the analysis presented.
Comparison between the sample considered in the present study $(\mathrm{n}=16937)$ and excluded participants $(n=7388)$ showed a substantial homogeneity for sex $(\mathrm{p}=0.23)$. The sample included was younger $(53 \pm 10.8 \mathrm{vs}$ $62 \pm 12.1 ; \quad \mathrm{p}<0.0001)$ and had higher social status $(p<0.0001)$, income $(p<0.0001)$ or education $(p<0.0001)$. Potential selection biases could emerge since the 36-Item Short Form Health Survey (SF-36) questionnaire was selfadministered and the topic not easily understandable by the elderly or people with a lower educational level or social status. In addition, we excluded subjects with CVD or cancer, which are medical conditions related to ageing and somehow to social status.

\section{Dietary information}

The validated Italian European prospective investigation into cancer and nutrition study food frequency questionnaire was used to evaluate food intake. ${ }^{22} 23$ The questionnaire, computerised with tailor-made software, allowed participants to be interviewed in an interactive way, by including illustrations of sample dishes of definite size or by reference to standard portion size. To simplify interpretation of data and to minimise within-person variations in intakes of individual foods, 188 food items were classified into 45 predefined food groups on the basis of similar nutrient characteristics or culinary usage (see online supplementary appendix 2 ).

Moderate alcohol intake was defined as regularly drinking no more than one drink a day by women, and no more than two drinks a day for men.

Food consumption patterns were generated by using Principal Components Analysis (PCA) conducted on the correlation matrix of 45 food groups. ${ }^{24}$ Three main factors emerged, in agreement with previous findings in the same population. ${ }^{24}$ The pattern identified as 'Olive Oil and Vegetables' was characterised by high positive loadings for olive oil, vegetables, legumes, soups, fruits and fish. The pattern named 'Meat and pasta' was characterised by high positive loadings for pasta, cooked tomatoes, red meat, animal fats and alcoholic beverages, and negative loadings of breakfast cereals and yogurt. The 'Eggs and Sweets' pattern was characterised by high positive loadings for eggs, margarines, processed meat and sugar and sweets.

We evaluated the adherence to MD by using the MD score (MDS) elaborated by Trichopoulou et al. ${ }^{25}$ Scoring was based on the intake of the following nine items: vegetables, legumes, fruit and nuts, dairy products, cereals, meat and meat products, fish, alcohol, and the ratio of monounsaturated:saturated fat. For most items, consumption above the study median received 1 point; all other intakes received 0 point. For dairy products, meat and meat products, consumption below the median received 1 point. Medians are gender specific. For ethanol, men who consumed $10-50 \mathrm{~g} /$ day and women who consumed $5-25 \mathrm{~g} /$ day received 1 point; otherwise, the score was 0 . The possible scores ranged between 0 and 9 , the latter reflecting the maximal adherence. 
We also used a newly proposed Italian Mediterranean diet index (IMI) whose score is based on the intake of 11 items: high intake of six typical Mediterranean foods (pasta; typical Mediterranean vegetables such as raw tomatoes, leafy vegetables, onion and garlic, salad, and fruiting vegetables; fruit; legumes; olive oil and fish); low intake of four non-Mediterranean foods (soft drinks, butter, red meat and potatoes) and alcohol consumption. If consumption of typical Mediterranean foods was in the third tertile of the distribution, the person received 1 point; all other intakes received 0 point. If consumption of non-Mediterranean foods was in the first tertile of the distribution, the person received 1 point. Ethanol received 1 point for intake up to $12 \mathrm{~g}$ /day; abstainers and persons who consumed $>12 \mathrm{~g} /$ day received 0 points. Possible scores ranged from 0 to $11 .{ }^{26}$ Such an Italian index was conceived to better capture healthy eating including foods more typically consumed in Italy.

The total FAC score was used to measure the antioxidant content from the diet. Its construction was already described in a previous study conducted within the Moli-sani cohort. ${ }^{27}$ Briefly, the content in the antioxidant vitamins and phytochemicals of each food group was derived by using the food composition tables from the Istituto Nazionale di Ricerca per gli Alimenti e la Nutrizione and the US Department of Agriculture (USDA). Healthy foods, according to an MD pattern, were categorised into either high or low antioxidant content. The total FAC score was constructed for a comparative evaluation of the consumption of these two groups. Coffee, chocolate and wine were not included in the analysis, despite their high antioxidant content, as their healthiness is reportedly limited to only moderate consumption. As the main antioxidant components of a healthy diet, the following items were considered: selenium, vitamins $\mathrm{C}, \mathrm{A}$ and $\mathrm{E}$, tocopherol- $\beta$, tocopherol- $\gamma$ and tocopherol- $\delta$, carotene $-\beta$ and carotene- $\alpha$, lycopene, lutein and all types of flavonoids.

Monounsaturated, saturated or polyunsaturated fatty acids and fibre intake from diet was expressed as $\mathrm{g} /$ day.

\section{Data collection}

Body mass index (BMI) was calculated as $\mathrm{kg} / \mathrm{m}^{2}$. Waist circumferences was measured according to the National Institute of Health, Heart, Lung and Blood guidelines. ${ }^{28}$ Blood pressure was measured by an automatic device (OMRON-HEM-705CP) three times on the non-dominant arm, with the patient lying down for about $5 \mathrm{~min}$. Hypertension was defined as systolic $\mathrm{BP} \geq 140 \mathrm{~mm} \mathrm{Hg}$ and/or diastolic $\mathrm{BP} \geq 90 \mathrm{~mm} \mathrm{Hg}$ or by the use of pharmacological treatment. ${ }^{29}$ Diabetes was defined if the glucose level was $\geq 126 \mathrm{mg} / \mathrm{dL}$ or by the use of pharmacological treatment. Hypercholesterolaemia status was defined if the total cholesterol level was $\geq 240 \mathrm{mg} / \mathrm{dL}$ or by the use of antihyperlipidemic treatment.

Physical activity was assessed by a structured questionnaire (24 questions on working and leisure time and sport participation) and expressed as daily energy expenditure in metabolic equivalent task-hours (MET/ day). Serum lipids and glucose were assayed by enzymatic reaction methods using an automatic analyser (ILab 350, Instrumentation Laboratory (IL), Milan, Italy). Low-density lipoprotein cholesterol was calculated according to Friedewald.

\section{Health-related quality of life and socioeconomic variables}

Health-related quality of life was assessed by using the validated Italian version of the self-administered SF-36. ${ }^{30}$ SF-36 is a general health scale that is widely used and thoroughly validated ${ }^{31}$ and has been translated into a number of languages, including Italian.

The questionnaire contains 36 items measuring eight multi-item parameters of health status covering the following domains: physical functioning, role limitations because of physical health problems, bodily pain, general health perceptions, vitality, social functioning, role limitations due to emotional problems and mental health. The first four domains deal with physical aspects and generate the physical component score, while the next four reflect psychological features and generate the mental component score.

For each domain, z-scores are calculated, summed and the sum transformed to a scale from 0 (the worst possible condition) to 100 (the best possible condition). ${ }^{32}$

In our study, the two scores expressed as continuous variables were then ranked into quartiles to allow specific statistical analyses.

Income categories were considered as low $(\leq 10000$ euros/year), low-medium (>10 000 $\leq 20000$ euros/year), medium-high $(>20000 \leq 40000$ euros/year $)$ and high (>40 000 euros/year).

Socioeconomic status (SES) was expressed as a score based on five variables: dwelling ownership and ratio between the number of rooms and the number of livingin family members (rooms per person), currently and at childhood, and the availability of hot water at home at childhood. The five components were dichotomised according to the median value, and a score of one was attributed to the category supposed to be a marker of higher social status in comparison with the opposite category: thus, we assigned a score of 1 to people living in a house with living-in family members/room density $>0.6$, dwelling ownership or with availability of hot water and a score of 0 to people with living-in family members/room density $\leq 0.6$, no dwelling ownership or with unavailability of hot water. The total SES score did not include either income or education.

Education level was divided into two categories: $\leq 8$ years of studies $(0$ point $)$ and $>8$ years of studies $(1$ point)

\section{STATISTICS}

Values for continuous variables are means \pm standard deviation (SD). Analysis of variance for continuous or categorical variables was applied to test the associations in table 1. Multivariable linear regression analysis was 
used for testing the association between mental and physical health scores (considered as continuous dependent variables) with adherence to MDS or dietary patterns (considered as continuous independent variables); linear regression coefficients were multiplied by $\mathrm{SD}$ of the independent variable. In this way, regression coefficients represent the variation in mental or physical scores for a $1 \mathrm{SD}$ change in independent variables, thus allowing comparability of the strength of the association among MDS, IMI or dietary patterns with mental and physical health.

By using multivariable logistic regression analysis, odds ratios (ORs) with a corresponding $95 \%$ confidence intervals (CI) were calculated to quantify the association of mental and physical health scores with adherence to dietary patterns. Four categories of adherence to MD were considered, ranging from low (0-2 points for MDS and IMI) to high ( $>6$ points for MDS and $\geq 5$ points for IMI) adherence. Dietary patterns from PCA were ranked into quartiles. For logistic regression analysis, the first quartiles of mental $(n=4234)$ or physical health were opposed to the fourth quartile $(n=4234)$. Participants with intermediate values (second and third quartiles) were excluded from this analysis to focus on the two extreme categories of self-reported health.

For linear and logistic regression analysis, potential confounders included as covariates in the models were age, sex, BMI, total energy intake, total physical activity, education, income, total socioeconomic status, smoking, diabetes, hypertension and hypercholesterolaemia. A specific category was created either for income or SES in order to account for missing values in the multivariable analyses.

Further adjustments for FAC, dietary fibre and fatty acids components were considered for testing their role in explaining the association among mental and physical health and MD markers.

The data analysis was generated using SAS/STAT software, V.9.1.3 of the SAS System for Windows 2009. SAS Institute Inc and SAS are registered trademarks of SAS Institute Inc, Cary, North Carolina, USA.

\section{RESULTS}

Table 1 shows the characteristics of the whole population according to the quartiles of mental and physical component scores. People in the uppermost quartile of selfreported mental health were $62.5 \%$ men and showed higher levels of leisure-time physical activity and a higher prevalence of high and medium-high income. Subjects in the highest quartile of physical health were younger, had lower BMI, as well as higher education, income and socioeconomic status. In addition, they had a lower prevalence of obesity, hypertension, diabetes and hypercholesterolaemia.

In table 2, the association between dietary scores and mental and physical health (considered as continuous variables) is reported.
Self-reported mental health was positively associated with greater adherence to MD in the fully adjusted model ( $\beta=0.33 ; 0.36 ; 0.50$ for MDS, IMI and olive oil and vegetables pattern, respectively) but inversely related to unhealthy dietary patterns $(\beta=-0.33$ for the eggs and sweets dietary pattern). The same positive association with MD was found for self-reported physical health, although the $\beta$-coefficients were lower than those observed for mental health $(\beta=0.15 ; 0.08$; 0.15 for MDS, IMI and olive oil and vegetables pattern). Conversely, physical health was negatively associated with the meat and pasta dietary pattern $(\beta=-0.11)$.

Multivariable logistic regression analysis showed that the odds of being in the uppermost level of mental health clearly increased according to the categories of adherence to the Mediterranean-like eating patterns (table 3). People in the highest category of adherence had either $42 \%$ (MDS) or $34 \%$ (IMI) or $59 \%$ (olive oil and vegetables pattern) statistically significant higher probabilities to be in the uppermost category of mental health than those in the lowest group of MD adherence (table 3).

Logistic regression analysis showed different results regarding physical health (table 4). Higher MD adherence was not associated with increased odds of being in the highest physical health category. Modest results were found only for the olive oil and vegetable pattern for which ORs of $22 \%$ for the highest category of adherence were detected $(p$ value $=0.01)$. However, physical health was inversely correlated with adherence to the meat and pasta pattern, (table 4) but positively correlated with the eggs and sweets pattern; in the latter case, people in the low-medium or highest quartile had $24 \%$ more chances to be in the top category of the physical component score (table 4).

MDS was positively associated with all four domains of mental health $(\beta=0.49$ and standard error $(\mathrm{SE}) \pm 0.06$; $\beta=0.25$ and $\mathrm{SE} \pm 0.06 ; \beta=0.16$ and $\mathrm{SE} \pm 0.06 ; \beta=0.38$ and $\mathrm{SE}$ \pm 0.07 ; for vitality, social functioning, role limitation and mental health, respectively, $\mathrm{p}<0.05$ for all) and with three out of four domains of physical health $(\beta=0.34$ and $\mathrm{SE} \pm 0.06 ; \beta=0.18$ and $\mathrm{SE} \pm 0.06 ; \beta=0.16$ and $\mathrm{SE} \pm 0.05$; for physical functioning, role limitation due to physical functioning and general health, respectively, $\mathrm{p}<0.05$ for all), whereas no association was found for bodily pain $(\beta=-0.01$ and $\mathrm{SE} \pm 0.03 ; \mathrm{p}=0.73$ ).

All the associations described above remained unchanged after further exclusion of subjects with diabetes $(n=1240 ; p<0.003$ for all $)$

\section{Dietary antioxidant, fibre intake and fatty acids content}

In this study, we also tested the possible accounting of key dietary components of MD for the association between dietary scores and mental or physical health.

The FAC score was positively associated with MDS $(\beta=0.27, \mathrm{SE} \pm 0.01 ; \mathrm{P}$ for trend $<0.0001 ; \beta=0.36, \mathrm{SE} \pm 0.01 ; \mathrm{P}$ for trend $<0.0001 ; \beta=0.20, \mathrm{SE} \pm 0.003 ; \mathrm{P}$ for trend $<0.0001$ for MDS, IMI and olive oil and vegetables pattern,

Bonaccio M, Di Castelnuovo A, Bonanni A, et al. BMJ Open 2013;3:e003003. doi:10.1136/bmjopen-2013-003003 


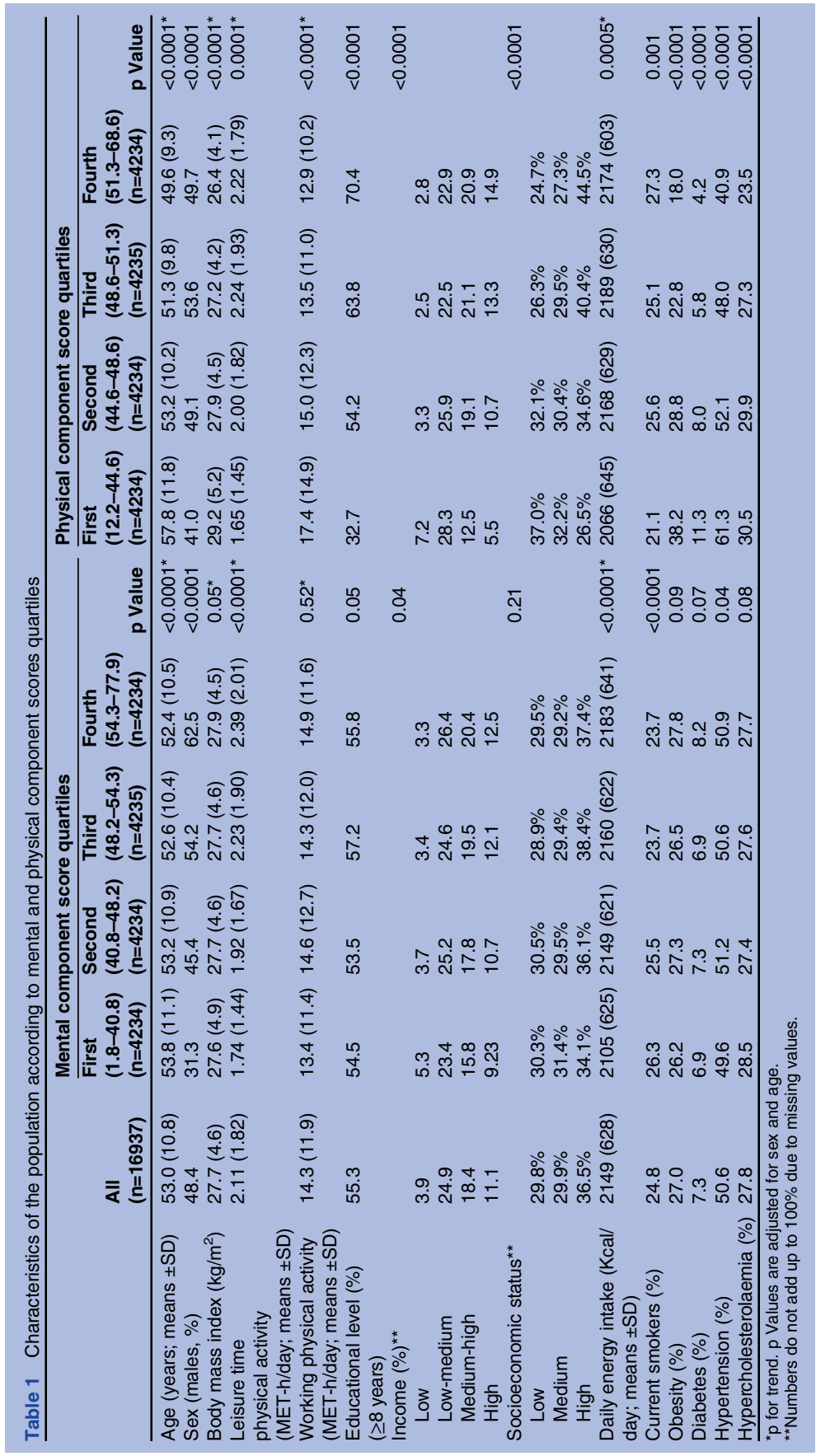

Bonaccio M, Di Castelnuovo A, Bonanni A, et al. BMJ Open 2013;3:e003003. doi:10.1136/bmjopen-2013-003003 


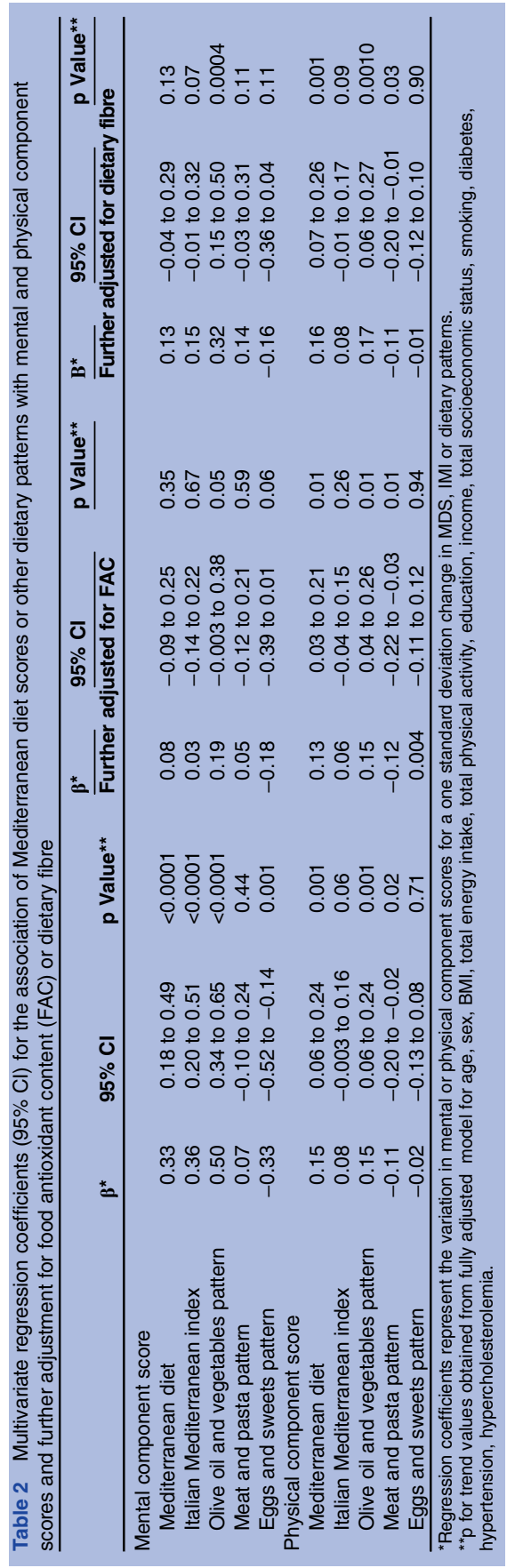

respectively) but inversely associated with the eggs and sweets pattern $(\beta=-0.04, \mathrm{SE} \pm 0.04 ; \mathrm{P}$ for trend $<0.0001)$, while no significant relationship was observed with the meat and pasta pattern $(\beta=0.005, \mathrm{SE} \pm 0.003$; $\mathrm{P}$ for trend= $0.16)$. The FAC score was significantly related to mental health $(\beta=0.25, \mathrm{SE} \pm 0.04$; $\mathrm{P}$ for trend $<0.0001)$ but not to physical health ( $\beta=0.002, \mathrm{SE} \pm 0.02$; $\mathrm{P}$ for trend $=0.92$ ).

The inclusion of the FAC score in the multivariable models abolished the association between MD and mental health (table 2). Similar results were obtained when the FAC score was included in the logistic regression analysis model (table 3 ).

Monounsaturated fatty acids were positively associated with mental health $(\beta=0.04, \mathrm{SE} \pm 0.01 ; \mathrm{P}$ for trend= $0.0004)$ but not with physical health $(\beta=0.01, \mathrm{SE} \pm 0.01$; $\mathrm{P}$ for trend $=0.06$ ).

Polyunsaturated fatty acids were positively associated with MDS ( $\beta=0.10 \mathrm{SE} \pm 0.01 ; p$ for trend $<0.0001 ; \beta=0.20$, $\mathrm{SE} \pm 0.01 ; \mathrm{p}$ for trend $<0.0001 ; \beta=0.22, \mathrm{SE} \pm 0.004 ; \mathrm{p}$ for trend $<0.0001$ for MDS, IMI and olive oil and vegetables pattern, respectively) and the eggs and sweets pattern $(\beta=0.19, \mathrm{SE} \pm 0.003$; $p$ for trend $<0.0001)$ but inversely linked to the meat and pasta pattern $(\beta=-0.07, \mathrm{SE} \pm 0.004$; $p$ for trend $<0.0001)$. No relationship was found between polyunsaturated fatty acids and mental $(\beta=0.03, \mathrm{SE} \pm 0.05$; $\mathrm{P}$ for trend $=0.52)$ or physical health $(\beta=-0.05, \mathrm{SE} \pm 0.03 ; \mathrm{P}$ for trend $=0.08$ ).

Saturated fatty acids were positively associated with physical health $(\beta=0.02, \mathrm{SE} \pm 0.01$; $\mathrm{P}$ for trend=0.006) but not with mental health $(\beta=-0.01, \mathrm{SE} \pm 0.01 ; \mathrm{P}$ for trend $=0.21)$.

The inclusion of dietary monounsaturated or saturated fatty acids in the multivariable model did not modify the association between MD and mental $(p<0.0001)$ or physical health $(p<0.05$ for all; for the IMI adjusted for monounsaturated fatty acids $p$ value $=0.17$ ) (see online supplementary table). Similarly, no changes were recorded after including dietary polyunsaturated fatty acids in the multivariate models ( $\mathrm{p}<0.05$ for both mental and physical health) (see online supplementary table).

Dietary fibre consumption was positively associated with MDS $(\beta=0.15, \mathrm{SE} \pm 0.002)$, IMI $(\beta=0.16, \mathrm{SE} \pm 0.002)$ and the olive oil and vegetable pattern $(\beta=0.08$, SE \pm 0.001 ; $p$ values $<0.0001$ for all) but inversely associated with the meat and pasta $(\beta=-0.02, \mathrm{SE} \pm 0.001 ; \mathrm{p}<0.0001)$ and the eggs and sweets patterns $(\beta=-0.04, \mathrm{SE} \pm 0.001$; $\mathrm{p}<0.0001)$. Fibre content was also positively associated with either mental health or less consistently with the physical component score $(\beta=0.11, \mathrm{SE} \pm 0.01, \mathrm{p}<0.0001$; $\beta=0.02, \mathrm{SE} \pm 0.01, \mathrm{p}=0.01$ respectively).

Once the fibre content from the diet was included in the fully adjusted model, the association between the Mediterranean-like eating patterns and mental health was clearly reduced for both MDS $(\beta=0.33, p<0.0001$ decreased to $\beta=0.13, p=0.13)$ and IMI $(\beta=0.36, p<0.0001$ decreased to $\beta=0.15, p=0.07$ ), suggesting indeed a role of dietary fibres for the explanation of the observed association between either MDS or IMI and better mental 


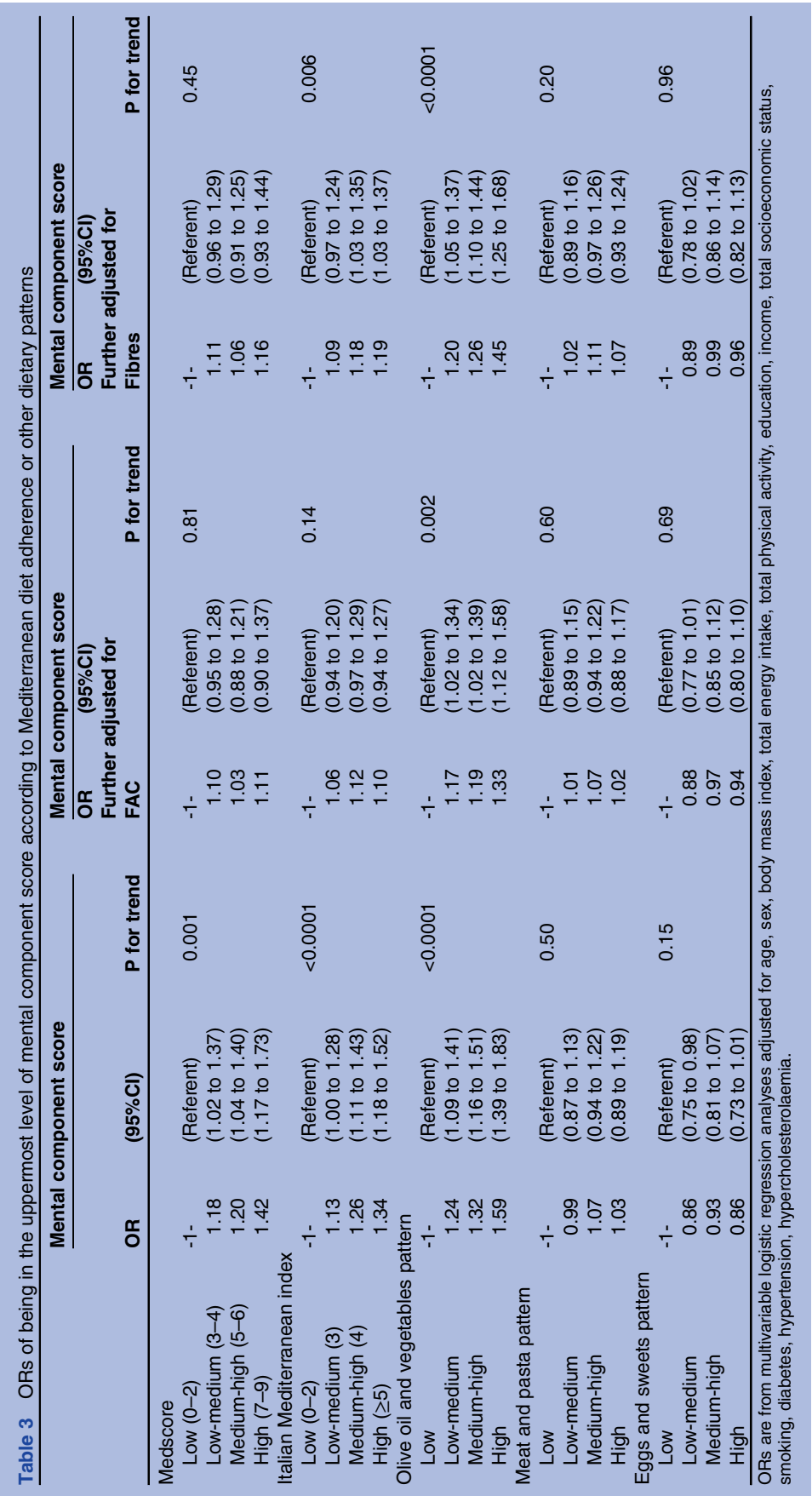

Bonaccio M, Di Castelnuovo A, Bonanni A, et al. BMJ Open 2013;3:e003003. doi:10.1136/bmjopen-2013-003003 


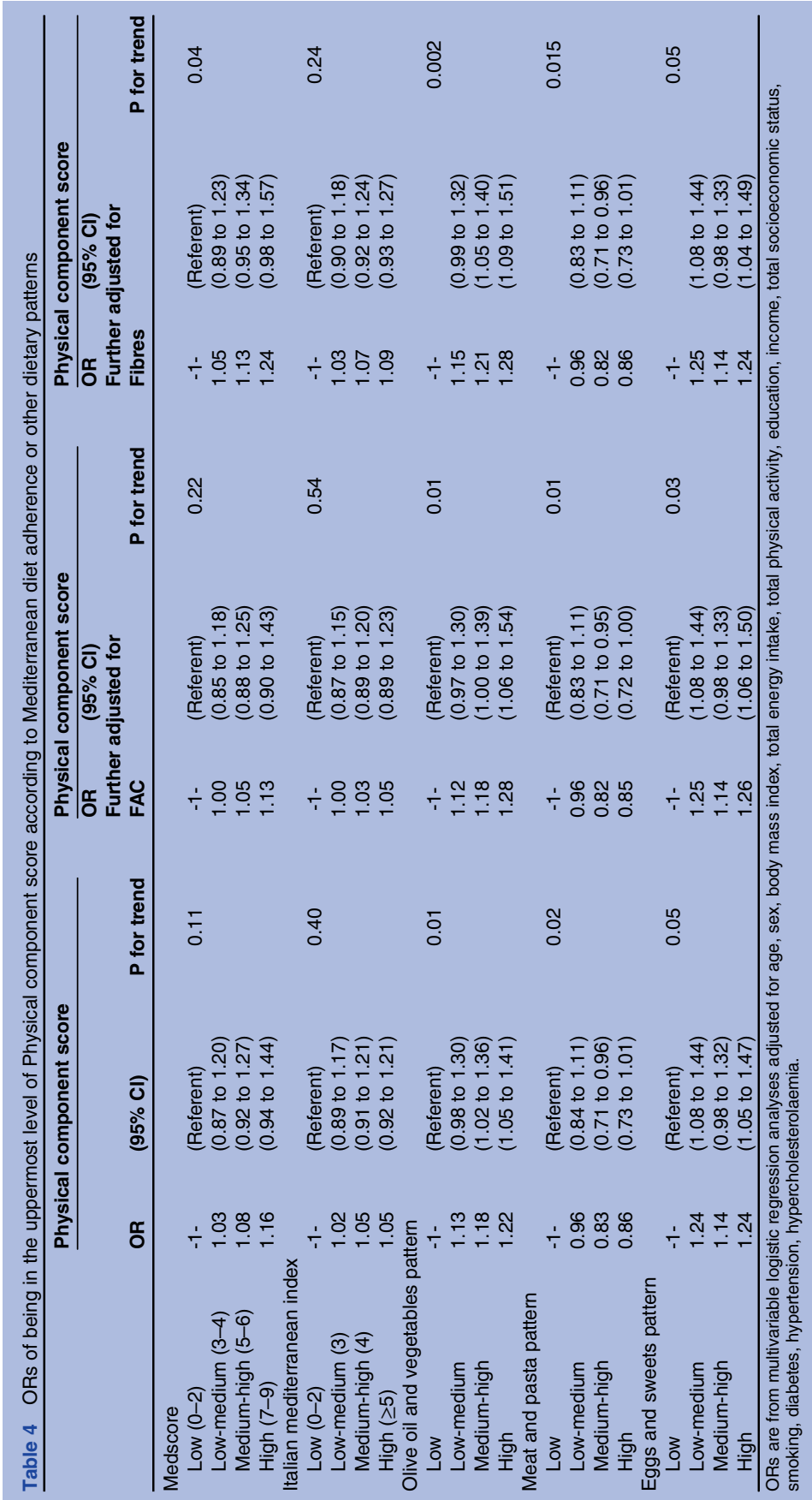

Bonaccio M, Di Castelnuovo A, Bonanni A, et al. BMJ Open 2013;3:e003003. doi:10.1136/bmjopen-2013-003003 
health (table 2). No significant changes were detected regarding the physical component score after adjustment for dietary fibre (table 2). The logistic regression analysis provided similar results (tables 3 and 4 ).

We performed further analysis by gender (data not shown) but failed to find any statistically significant sexrelated difference in the association of mental or physical scores with MDS or other dietary patterns as measured by specific terms of interaction (all $\mathrm{p}>0.05$ ).

\section{DISCUSSION}

In this large sample of a general adult population from the Moli-sani study, health-related quality of life was associated with high adherence to Mediterranean-like eating patterns and inversely related to unhealthy dietary habits. Our data agree with previous findings within Mediterranean cohorts, ${ }^{13}{ }^{14}$ although in our study the relationship with MD was more consistent for mental health than for physical health.

Adherence to an MD pattern has been positively associated with reduced risk for a wide range of chronic diseases, including neurodegenerative disorders ${ }^{1}$ and cognitive decline, ${ }^{33} 34$ which are closely linked to selfperceived mental health. ${ }^{35} 36$ The beneficial effects of MD have been ascribed to its essential components. Antioxidants and polyphenols, largely present in Mediterranean foods, such as plant foods, fruits, olive oil and red wine, have anti-inflammatory properties and have been proven to exert a positive role against cardiovascular diseases and cancer. ${ }^{57}$ Also, monounsaturated fatty acids intake, whose major source is represented by olive oil, was found to be associated with a reduced prevalence of risk factors for major chronic disease, ${ }^{38} 39$ while a higher consumption of dietary fibre has beneficial effects on CVD risk reduction and is linked to a lower risk of fatal ischaemic heart disease. ${ }^{18} 19$ In the wake of this evidence, we sought to examine whether the positive relationship between higher adherence to MD patterns and highest HRQL could be accounted for by the total antioxidant content from diet or by other compounds such as monounsaturated or polyunsaturated fatty acids or dietary fibre.

Higher intake of dietary antioxidants accounted for the positive association between MD adherence and higher mental status. In contrast, the levels of dietary antioxidants were not associated with physical health and thus failed to explain the observed relationship between higher adherence to MD and better self-reported physical status. Dietary fibre too was positively linked to improved mental health and apparently contributed to the explanation of the positive association with MD, however in a less consistent way as compared to the antioxidant intake. As observed with the antioxidant content, dietary fibre did not account for the association between physical health and higher adherence to MD.

Conversely, neither monounsaturated nor polyunsaturated fatty acids from the diet modified the association between MD and better quality of life. However, data on polyunsaturated fatty acids should be taken with precaution since we could not distinguish the two major classes of polyunsaturated fatty acids (omega-3 or omega-6), which have opposing biological activities.

Previous studies have investigated the role of the basic components of MD on quality of life finding direct associations between some of its components and HRQL, such as fish consumption with mental health ${ }^{40}$ or with self-reported physical health. ${ }^{41}$ Inverse associations were also found between fruit and vegetables consumption and incidence of depressive symptoms. ${ }^{42}$ Yet this kind of approach fails to consider diet as a whole, most likely ending up with underestimating the interactions among several foods and nutrients. ${ }^{43}$ In addition to using a more traditional tool to evaluate adherence to healthy habits by using two a priori scores, our study approached overall diet as a combination of healthy and less healthy foods with an a posteriori approach which is able to catch the real dietary habits of people and accounts for correlations between score components.

Although previous studies had provided interesting data on the direct association between MD and better HRQL, ${ }^{14}$ so far they have not approached the issue of possible mediators that are able to explain the observed beneficial effects of MD on self-reported health status.

Positive associations between antioxidants intake and cognitive function have been largely documented ${ }^{44} 45$ and were mainly ascribed to the preventing and retarding activity of antioxidants on the uncontrolled production of free radicals that might yield beneficial effects on the frontal/subcortical brain systems, with likely enhancement of cognitive functions. ${ }^{34}$ Oxidative stress has been implicated in the pathophysiology of many neuropsychiatric disorders ${ }^{46}$ such as schizophrenia, bipolar disorder ${ }^{47}$ and major depression. ${ }^{48}$

Recent epidemiological studies have confirmed a correlation between diet and depression, pointing out the importance of a diet rich in antioxidants and other essential compounds typical of MD in reducing the risk of depression, which may explain the positive effects in improving mental health. ${ }^{49}$

Regarding physical health, there are several biological and physiological mechanisms that could explain the beneficial effects of an MD pattern, such as reduced vascular inflammation and coagulation markers or improved endothelial function, ${ }^{50} 51$ all factors that are likely to be related to a better physical health status. However, in our study, neither the antioxidant content of diet nor the dietary fibre intake affected the association between Mediterranean scores and physical health. Improved physical health was also associated with higher adherence to the eggs and sweets pattern, characterised by high positive loadings of eggs, margarines, processed meat and sugar and sweets. Despite being rich in less healthy foods, this pattern has a high content of polyunsaturated fatty acids that could explain the positive relationship with improved physical health, as 
suggested by previous studies. ${ }^{40}$ However, the inclusion of polyunsaturated fatty acids in the model did not change this association. In the absence of any valid food compound that is able to explain the association between physical health and the eggs and sweets pattern, we cannot exclude a possible reverse-causality effect. Indeed, this eating pattern is mainly adopted by young people who are more confident about their physical health and thus could be more likely to disregard healthy dietary patterns. Although the data were age adjusted, a possible residual confounding due to age and other unmeasured age-related conditions cannot be excluded.

\section{Strengths of this study}

This work represents one of the few studies investigating the relationship between dietary patterns and health-related quality of life in a Mediterranean country. An additional strength is the large sample used for testing the association. In addition, it is apparently the first study to provide a likely account of antioxidants from the diet for the direct association between MD and HRQL to address this topic by using simultaneously two a priori Mediterranean scores and the a posteriori dietary patterns obtained by principal component analysis.

\section{Limitations of this study}

The present study was cross-sectional and shares all the limitations of this study type; in particular, inference of possible causality is unwarranted since it is not possible to determine whether the diet promotes well-being or whether those who 'feel better' choose to eat a healthy diet. However, in order to account for this reverse causality bias, we excluded from the analysis subjects with previous cancer, cardiovascular disease or diabetes as these conditions may have led to changes in their diet. We measured the overall intake of antioxidants by using a score based on estimates of micronutrient intake from the food frequency questionnaire. We recognise that this approach is challenging and that our indirect antioxidant intake assessment could have lower accuracy than direct measurements, which conversely are more difficult to perform in a very large sample size population.

Caution is needed in extending the results presented here to larger contexts since the data were collected in a region located between central and southern Italy, Mediterranean by tradition and culture. ${ }^{17}$ Yet, the main characteristics of our sample are comparable to those of the Italian Cardiovascular Epidemiological Observatory, ${ }^{52}$ a large survey including random samples of the general population all over Italy; therefore, our sample could be considered to be representative at least of the Italian population.

\section{CONCLUSIONS}

Our data indicate a positive association between adherence to the Mediterranean diet and self-reported mental and physical health status. The association is more obvious for mental than for physical health.

This large cross-sectional study has offered interesting indications on this issue by highlighting a major role played by dietary antioxidants in explaining the relationship between MD and HRQL. This is important in order to advance the hypothesis on the cause-effect relationship between MD and HRQL and to plan new strategies for the prevention of mental health decline in the general population.

\section{Author affiliations}

${ }^{1}$ Laboratory of Genetic and Environmental Epidemiology, Laboratori di Ricerca, Fondazione di Ricerca e Cura "Giovanni Paolo II", Università Cattolica del Sacro Cuore, Campobasso, Italy

${ }^{2}$ Department of Epidemiology and Prevention, IRCCS Istituto Neurologico Mediterraneo Neuromed, Pozzilli, Isernia, Italy

${ }^{3}$ Epicomed Research Srl, Campobasso, Italy

${ }^{4}$ Laboratory of Cardiovascular and Neurovascular Epidemiology, Casa di Cura Montevergine, Mercogliano, Avellino, Italy

Contributors $\mathrm{MB}, \mathrm{ADC}$ and $\mathrm{LI}$ designed the research; $\mathrm{FDL}, \mathrm{MB}, \mathrm{SC}$ and FZ managed the data collection; $\mathrm{MB}$ and $\mathrm{ADC}$ analysed the data; $\mathrm{MB}$ and $\mathrm{AB}$ wrote the paper; GP developed the FAC score; MBD, GdG and LI originally inspired the research, obtained the financial support and critically reviewed the manuscript. All authors have read and approved the final version of the manuscript.

Funding The enrolment phase of the Moli-sani Project was supported by research grants from the Pfizer Foundation (Rome, Italy), Instrumentation Laboratory (IL, Milan, Italy) and the Italian Ministry of University and Research (MIUR, Rome, Italy)-Programma Triennale di Ricerca, Decreto no.1588. The Pfizer Foundation, IL and MIUR had no role in the study design, collection, analysis, and interpretation of data; in the writing of the report; and in the decision to submit the article for publication.

\section{Competing interests None.}

Ethics approval The Moli-sani study was approved by the Ethics Committee of the Catholic University of Rome. Participants signed the informed consent before taking part in the study.

Provenance and peer review Not commissioned; externally peer reviewed.

Data sharing statement № additional data are available.

Open Access This is an Open Access article distributed in accordance with the Creative Commons Attribution Non Commercial (CC BY-NC 3.0) license, which permits others to distribute, remix, adapt, build upon this work noncommercially, and license their derivative works on different terms, provided the original work is properly cited and the use is non-commercial. See: http:// creativecommons.org/licenses/by-nc/3.0/

\section{REFERENCES}

1. Sofi F, Abbate R, Gensini GF, et al. Accruing evidence on benefits of adherence to the Mediterranean diet on health: an updated systematic review and meta-analysis. Am J Clin Nutr 2010:92:1189-96.

2. Trichopoulou A, Bamia C, Trichopoulos D. Anatomy of health effects of Mediterranean diet: Greek EPIC prospective cohort study. BMJ 2009;338:b2337

3. Bonaccio M, lacoviello L, de Gaetano G, On Behalf Of The Moli-Sani Investigators. The Mediterranean diet: the reasons for a success. Thromb Res 2012;129:401-4.

4. Trichopoulou A, Vasilopoulou E, Lagiou A. Mediterranean diet and coronary heart disease: are antioxidants critical? Nutr Rev 1999;57:253-5.

5. Giacosa A, Barale R, Bavaresco $L$, et al. Cancer prevention in Europe: the Mediterranean diet as a protective choice. Eur $\mathrm{J}$ Cancer Prev 2013;22:90-5.

6. Mattioli AV, Miloro C, Pennella S, et al. Adherence to Mediterranean diet and intake of antioxidants influence spontaneous conversion of atrial fibrillation. Nutr Metab Cardiovasc Dis 2013;23:115-21. 
7. Karr JE, Alexander JE, Winningham RG. Omega-3 polyunsaturated fatty acids and cognition throughout the lifespan: a review. Nutr Neurosci 2011;14:216-25.

8. Baumann C, Erpelding ML, Perret-Guillaume C, et al. Health-related quality of life in French adolescents and adults: norms for the DUKE Health Profile. BMC Public Health 2011:11:401.

9. Bardage C, Isacson D, Pedersen NL. Self-rated health as a predictor of mortality among persons with cardiovascular disease in Sweden. Scand J Public Health 2001;29:13-22.

10. Idler EL, Benyamini $Y$. Self-rated health and mortality: a review of twenty-seven community studies. J Health Soc Behav 1997;38:21-7.

11. Heidrich J, Liese AD, Löwel $\mathrm{H}$, et al. Self-rated health and its relation to all-cause and cardiovascular mortality in southern Germany. Results from the MONICA Augsburg cohort study 1984-1995. Ann Epidemiol 2002;12:338-45.

12. Nery Guimarães JM, Chor D, Werneck GL, et al. Association between self-rated health and mortality: 10 years follow-up to the Pró-Saúde cohort study. BMC Public Health 2012;12:676.

13. Sánchez PH, Ruano C, de Irala J, et al. Adherence to the Mediterranean diet and quality of life in the SUN Project. Eur J Clin Nutr 2012;66:360-8.

14. Muñoz MA, Fíto M, Marrugat $\mathrm{J}$, et al. Adherence to the Mediterranean diet is associated with better mental and physical health. Br J Nutr 2009;101:1821-7.

15. Costarelli V, Koretsi E, Georgitsogianni E. Health-related quality of life of Greek adolescents: the role of the Mediterranean diet. Qual Life Res 2013;22:951-6.

16. Sánchez-Villegas A, Delgado-Rodríguez M, Alonso A, et al. Association of the Mediterranean dietary pattern with the incidence of depression: the Seguimiento Universidad de Navarra/University of Navarra follow-up (SUN) cohort. Arch Gen Psychiatry 2009;66:1090-8.

17. Rienks J, Dobson AJ, Mishra GD. Mediterranean dietary pattern and prevalence and incidence of depressive symptoms in mid-aged women: results from a large community-based prospective study. Eur J Clin Nutr 2013;67:75-8.

18. Satija A, Hu FB. Cardiovascular benefits of dietary fiber. Curr Atheroscler Rep 2012;14:505-14.

19. Crowe FL, Key TJ, Appleby PN, et al. Dietary fibre intake and ischaemic heart disease mortality: the European Prospective Investigation into Cancer and Nutrition-Heart study. Eur J Clin Nutr 2012;66:950-6.

20. Ajala O, English P, Pinkney J. Systematic review and meta-analysis of different dietary approaches to the management of type 2 diabetes. Am J Clin Nutr 2013;97:505-16.

21. Bonaccio M, Bonanni AE, Di Castelnuovo $A$, et al. Low income is associated with poor adherence to a Mediterranean diet and a higher prevalence of obesity: cross-sectional results from the Moli-sani study. BMJ Open 2012;2:e001685. doi:10.1136/bmjopen2012-001685

22. Pala V, Sieri S, Palli D, et al. Diet in the Italian EPIC cohorts: presentation of data and methodological issues. Tumori 2003;89:594-607.

23. Pisani P, Faggiano F, Krogh V, et al. Relative validity and reproducibility of a food frequency dietary questionnaire for use in the Italian EPIC centres. Int J Epidemiol 1997;26(Suppl 1):S152-60.

24. Centritto $F$, lacoviello L, di Giuseppe R, et al. Dietary patterns, cardiovascular risk factors and C-reactive protein in a healthy Italian population. Nutr Metab Cardiovasc Dis 2009;19:697-706.

25. Trichopoulou A, Costacou T, Bamia C, et al. Adherence to a Mediterranean diet and survival in a Greek population. $N$ Engl J Med 2003;348:2599-60819.

26. Agnoli C, Krogh V, Grioni S, et al. A priori-defined dietary patterns are associated with reduced risk of stroke in a large Italian cohort. $J$ Nutr 2011;141:1552-8.

27. Pounis G, Costanzo S, di Giuseppe R, et al. Consumption of healthy foods at different content of antioxidant vitamins and phytochemicals and metabolic risk factors for cardiovascular disease in men and women of the Moli-sani study. Eur J Clin Nutr 2013;67:207-13.

28. Janssen I, Katzmarzyk PT, Ross R. Body mass index, waist circumference, and health risk: evidence in support of current National Institutes of Health guidelines. Arch Intern Med 2002;162:2074-9.

29. Guidelines Committee. 2003 European Society of HypertensionEuropean Society of Cardiology guidelines for the management of arterial hypertension. J Hypertens 2003;21:1011-53.
30. Apolone G, Mosconi P. The Italian SF-36 Health Survey: translation, validation and norming. J Clin Epidemiol 1998;51:1025-36.

31. Ware JE Jr, Gandek B. Overview of the SF-36 Health Survey and the International Quality of Life Assessment (IQOLA) Project. J Clin Epidemiol 1998;51:903-12.

32. Ware J, Sherbourne CD. The MOS 36-item short form health survey (SF-36). 1. Conceptual frame-work and item selection. Med Care 1992;30:473-81.

33. Naqvi AZ, Harty B, Mukamal KJ, et al. Monounsaturated, trans, and saturated fatty acids and cognitive decline in women. J Am Geriatr Soc 2011;59:837-43.

34. Panza F, Solfrizzi V, Colacicco AM, et al. Mediterranean diet and cognitive decline. Public Health Nutr 2004;7:959-63.

35. Visser M, Verbaan D, van Rooden S, et al. A longitudinal evaluation of health-related quality of life of patients with Parkinson's disease. Value Health 2009;12:392-6.

36. Karlsen $\mathrm{KH}$, Tandberg E, Arsland D, et al. Health related quality of life in Parkinson's disease: a prospective longitudinal study. $J$ Neurol Neurosurg Psychiatry 2000;69:584-9.

37. Scoditti E, Calabriso N, Massaro M, et al. Mediterranean diet polyphenols reduce inflammatory angiogenesis through MMP-9 and COX-2 inhibition in human vascular endothelial cells: a potentially protective mechanism in atherosclerotic vascular disease and cancer. Arch Biochem Biophys 2012;527:81-9.

38. Brehm BJ, Lattin BL, Summer SS, et al. One-year comparison of a high-monounsaturated fat diet with a high-carbohydrate diet in type 2 diabetes. Diabetes Care 2009;32:215-20.

39. Baum SJ, Kris-Etherton PM, Willett WC, et al. Fatty acids in cardiovascular health and disease: a comprehensive update. J Clin Lipidol 2012;6:216-34.

40. Silvers KM, Scott KM. Fish consumption and self-reported physical and mental health status. Public Health Nutr 2002;5:427-31.

41. Schiepers OJ, de Groot RH, Jolles J, et al. Fish consumption, no fatty acid status, is related to quality of life in a healthy population. Prostaglandins Leukot Essent Fatty Acids 2010;83:31-5.

42. Liu C, Xie B, Chou CP, et al. Perceived stress, depression and food consumption frequency in the college students of China Seven Cities. Physiol Behav 2007:92:748-54.

43. Hu FB. Dietary pattern analysis: a new direction in nutritional epidemiology. Curr Opin Lipidol 2002;13:3-9.

44. Jama JW, Launer LJ, Witteman JC, Dietary antioxidants and cognitive function in a population-based sample of older persons. The Rotterdam study. Am J Epidemiol 1996;144 275-80.

45. Valls-Pedret C, Lamuela-Raventós RM, Medina-Remón A, et al. Polyphenol-rich foods in the Mediterranean diet are associated with better cognitive function in elderly subjects at high cardiovascular better cognitive function in elderly subjects
risk. J Alzheimers Dis 2012;29:773-82.

46. $\mathrm{Ng} \mathrm{F}$, Berk M, Dean O, et al. Oxidative stress in psychiatric disorders: evidence base and therapeutic implications. Int $J$ Neuropsychopharmacol 2008;11:851-76.

47. Andreazza AC, Kauer-Sant'anna M, Frey BN, et al. Oxidative stress markers in bipolar disorder: a meta-analysis. J Affect Disord 2008;111:135-44.

48. Maes M, De Vos N, Pioli R, et al. Lower serum vitamin $\mathrm{E}$ concentrations in major depression. Another marker of lowered antioxidant defenses in that illness. J Affect Disord 2000;58: 241-6.

49. Jacka FN, Mykletun A, Berk M. Moving towards a population health approach to the primary prevention of common mental disorders. BMC Med 2012;10:149.

50. Esposito K, Marfella R, Ciotola M, et al. Effect of a Mediterranean-style diet on endothelial dysfunction and markers of vascular inflammation in the metabolic syndrome: a randomized trial. JAMA 2004;292:1440-6.

51. Chrysohoou C, Panagiotakos DB, Pitsavos C, et al. Adherence to the Mediterranean diet attenuates inflammation and coagulation process in healthy adults: the ATTICA study. J Am Coll Cardiol 2004;44:152-8.

52. Giampaoli S, Rielli R, Dematté L, et al. The Italian observatory of cardiovascular risk: the CUORE project experience [abstract]. Circulation 2009;119:139. 


\section{CHAPTER 4.}

Adherence to the Mediterranean diet is associated with lower platelet and leukocyte counts: results from the Moli-sani study

Bonaccio M, Di Castelnuovo A, De Curtis A, Costanzo S, Persichillo M, Donati MB, Cerletti C, Iacoviello L and de Gaetano G.

Blood. 2014;123:3037-44. 


\section{Regular Article}

\section{PLATELETS AND THROMBOPOIESIS}

\section{Adherence to the Mediterranean diet is associated with lower platelet and leukocyte counts: results from the Moli-sani study}

Marialaura Bonaccio, Augusto Di Castelnuovo, Amalia De Curtis, Simona Costanzo, Mariarosaria Persichillo, Maria Benedetta Donati, Chiara Cerletti, Licia lacoviello, and Giovanni de Gaetano, on behalf of the Moli-sani Project Investigators

Department of Epidemiology and Prevention, Istituto Di Ricovero e Cura a Carattere Scientifico Istituto Neurologico Mediterraneo Neuromed, Pozzilli, Italy

\section{Key Points}

- Adherence to a Mediterranean diet is associated with reduced platelet and leukocyte counts.

- The observed associations are partially explained by the high dietary fiber and antioxidant content of the Mediterranean diet.
Platelet (PLT) and white blood cell (WBC) counts are 2 markers of inflammation and have been linked to the risk for cerebrovascular and coronary heart disease. A Mediterranean diet (MD) has been associated with reduced inflammation and mortality for major chronic diseases. We aimed at evaluating the association between the MD and both PLT and WBC counts. This cross-sectional analysis in a population-based cohort study included 14586 healthy Italian citizens enrolled within the Moli-sani study. Adherence to MD was appraised by either the MD Score (MDS) or the Italian Mediterranean Index (IMI). PLT and WBC counts were both inversely related to MD adherence (MDS: $P<.0001$ and $P=.008$, respectively). As compared with those with poorer $M D$ adherence, subjects with greater adherence had both reduced odds of being in the highest PLT-count group (MDS: odds ratio $=0.50 ; 95 \%$ confidence interval, $0.31-0.80$ ) and increased odds of being in the lowest WBC-count group (IMI: odds ratio $=1.41 ; 95 \%$ confidence interval, 1.07-1.86). The association between WBC count and MDS disappeared when further adjusted for PLT count, whereas the association between PLT count and the MD was not affected by adjustment for WBCs. Food antioxidant and dietary
fiber content modified the inverse association between MDS and WBC count and partially accounted for the association with PLTs. fiber content modified the invers

\section{Introduction}

Increased platelet (PLT) and white blood cell (WBC) counts have been recognized as possible markers of inflammation and of greater risk for cerebrovascular and coronary heart disease. In particular, PLT count has been associated with vascular ${ }^{1}$ and nonvascular death, including cancer. ${ }^{2}$ WBC count is a predictor of fatal and nonfatal ischemic vascular disease independent of other traditional cardiovascular disease (CVD) risk factors ${ }^{3,4}$ and is a broadly used marker of systemic inflammation. ${ }^{5}$

Conversely, a Mediterranean dietary pattern has been associated with a reduction of low-grade inflammation ${ }^{6}$ and mortality for cardiovascular and neurodegenerative diseases. ${ }^{7}$ The Mediterranean diet (MD) is an eating pattern typical of the Mediterranean basin and is characterized by a wide consumption of plant foods, cereals, legumes, fish, olive oil as main fat source, and moderate (red) wine consumption. ${ }^{8}$

The beneficial effects of MD on health have been ascribed to its high content of antioxidants, fiber, monounsaturated fatty acids, and polyunsaturated fatty acids (PUFAs). ${ }^{9}$ In particular, antioxidants and polyphenols have been shown to exert a positive role against ischemic vascular disease mainly due to their anti-inflammatory properties. ${ }^{10,11}$

Dietary fiber intake was associated with benefits for cardiovascular health and reduced risk of cardiovascular mortality ${ }^{12,13}$;

Submitted November 29, 2013; accepted March 5, 2014. Prepublished online as Blood First Edition paper, March 31, 2014; DOI 10.1182/blood-2013-12541672. similarly, a balanced ratio of $\omega-6$ and $\omega-3$ essential fatty acids has been suggested to yield favorable effects on cardio- and cerebrovascular health. ${ }^{14}$

The aim of this study was to investigate the association of an MD with PLT or WBC count based on the hypothesis that a diet rich in healthy compounds could favorably influence these 2 cellular biomarkers of low-grade inflammation in subjects without any overt chronic or hematologic disease.

Because adherence to the MD and both cell counts appeared to be inversely associated, we tested the possible role played by food antioxidant content (FAC), dietary fiber, and PUFA intake in accounting for the observed relationship.

\section{Methods}

\section{Study population}

A population-based cohort of 24325 citizens of Molise, a region located between central and southern Italy, were enrolled in the Moli-sani study. Between March 2005 and April 2010, men and women aged $\geq 35$ years were randomly recruited from subjects included in the city-hall registries of Molise. ${ }^{15}$ marked "advertisement" in accordance with 18 USC section 1734.

C 2014 by The American Society of Hematology 
For the present study, individuals from the Moli-sani cohort reporting a baseline a personal history of CVD (angina, myocardial infarction, heart failure, revascularization procedures, and stroke; $6.0 \%$ ) or cancer $(3.1 \%)$, those for whom there were no data on PLT $(2.7 \%)$ or WBC count $(2.7 \%)$, those for whom there were unreliable dietary or medical questionnaires $(3.9 \%$ and $1 \%$ ) or missing values for the FAC score (3.5\%), those reporting hepatiti $\mathrm{B}$ or $\mathrm{C}$ or an hematologic disease $(2.9 \%$ and $2.2 \%$, respectively), and in dividuals recruited at the site of Termoli (due to a different cell counter used $\mathrm{n}=5107 ; 21.0 \%$ ) were excluded from the analysis. The final sample comprised 14586 individuals. Comparison between the whole Moli-sani cohor $(n=24325)$ and the analyzed sample showed homogeneity for sex, PLT and WBC counts, and both the dietary scores (all $P$ values $>.05$ ), but the sample was slightly younger $(54.2 \pm 11.5$ vs $55.8 \pm 12 ; P<.0001)$.

The Moli-sani study was approved by the ethics committee of the Catholic University of Rome. All participants signed an informed consent before taking part in the study. This study was conducted in accordance with the Declaration of Helsinki.

\section{Dietary information}

Food intake was determined by the validated Italian version of the "European project investigation into cancer and nutrition" food frequency questionnaire. ${ }^{16,17}$ The questionnaire, computerized with tailor-made software, allowed us to interview participants in an interactive way, including illustrations of sample dishes of definite sizes or by reference to standard portion sizes. To simplify the interpretation of data and to minimize withinperson variations in intake of individual foods, 188 food items were classified into 45 predefined food groups on the basis of similar nutrient characteristics or culinary usage.

We evaluated the adherence to the MD by using the MD Score (MDS) developed by Trichopoulou et al. ${ }^{18}$ Briefly, scoring was calculated in a population free from CVD, cancer, or diabetes and was based on the intake of the following 9 items: vegetables, legumes, fruits and nuts, dairy products, cereals, meat and meat products, fish, alcohol, and monounsaturated to saturated fat ratio. For most items, consumption above the study median received 1 point; all other intakes received 0 points. For dairy products, meat and meat products, consumption below the median received 1 point. Medians were gender specific. For ethanol, men who consumed 10 to $50 \mathrm{~g} /$ day an women who consumed 5 to $25 \mathrm{~g} /$ day received 1 point; otherwise, the score wa 0 . The possible scores ranged between 0 and 9 , the latter reflecting the maximal adherence to the MD.

In addition, we used the Italian Mediterranean Index (IMI), recently proposed to better capture some healthy foods more typically consumed in Italy, such as pasta. ${ }^{19}$ This IMI score was based on the intake of 11 items: high intake of 6 typical Mediterranean foods (pasta; typical Mediterranean veg etables such as raw tomatoes, leafy vegetables, onion and garlic, salad, and fruiting vegetables; fruit; legumes; olive oil; and fish), low intake of 4 nonMediterranean foods (soft drinks, butter, red meat, and potatoes), and alcoho consumption. If consumption of typical Mediterranean foods was in the thir tertile of the distribution, the person received 1 point; all other intakes received 0 points. If consumption of non-Mediterranean foods was in the first tertile of the distribution, the person received 1 point. Ethanol received 1 point for intake up to $12 \mathrm{~g} /$ day; abstainers and persons who consumed $>12 \mathrm{~g} /$ day received 0 points. Possible scores ranged from 0 to 11 , the latter reflecting the maximal adherence to MD. ${ }^{19}$

Total FAC score was used to measure the antioxidant content of the diet, as previously described. ${ }^{20}$ Briefly, the content in antioxidant vitamin and phytochemicals of each food group was derived by using the food composition tables from the Italian Istituto Nazionale di Ricerca per gl Alimenti e la Nutrizione and the US Department of Agriculture. Healthy foods, according to a Mediterranean eating pattern, were categorized into either high or low antioxidant content. The total FAC score was constructed for a comparative evaluation of the consumption of these 2 groups. Coffee, chocolate, and wine were not included in the analysis, despite thei high antioxidant content, because their healthiness is reportedly limited to moderate consumption only. ${ }^{21}$ The following items were considered a main antioxidant components of a healthy diet: selenium; vitamins $\mathrm{C}, \mathrm{A}$ and $\mathrm{E}$; tocopherol- $\beta,-\gamma$, and $-\delta$; carotene- $\beta$ and $-\alpha$; lycopene; lutein; and all types of flavonoids. PUFAs (evaluated as linoleic acid $\omega-6$ to linolenic acid $\omega-3$ ratio) or fiber intake from the diet was expressed as $\mathrm{g} /$ day.

\section{Anthropometric measurements and assessment of risk factors}

Body mass index (BMI) was calculated as $\mathrm{kg} / \mathrm{m}^{2}$. Waist circumferences were measured according to the National Heart, Lung and Blood Institute guidelines. ${ }^{22}$ High blood pressure (BP) was defined as systolic BP $\geq 140 \mathrm{~mm}$ $\mathrm{Hg}$ and/or diastolic $\mathrm{BP} \geq 90 \mathrm{~mm} \mathrm{Hg}$ or by pharmacologic treatment. ${ }^{23}$ Hyperglycemia was defined if glucose level was $\geq 126 \mathrm{mg} / \mathrm{dL}$ or by pharmacologic treatment. Hypercholesterolemia was defined if total cholesterol level was $\geq 240$ or by antihyperlipidemic treatment. C-reactive protein (CRP) was measured by a high-sensitivity assay (IL-Coagulation Systems ACL9000; IL, Milan, Italy).

Subjects were classified as nonsmokers, ex-smokers (having smoked cigarettes in the past and having quitted for at least 1 year), and current smokers. ${ }^{24}$

Physical activity was assessed by a structured questionnaire ( 24 questions on working and leisure time and sport participation) and expressed as daily energy expenditure in metabolic equivalent task-hours.

Global individual cardiovascular risk was calculated applying the risk equations of the CUORE project. ${ }^{25}$ It predicts the 10 -year risk of vascular fatal or nonfatal events. For men, the cardiovascular risk was categorized as low $(<3 \%)$, medium $(\geq 3 \%$ and $<20 \%)$, or high $(\geq 20 \%)$ risk. For women, low CVD risk was set at $<3 \%$, whereas the high-risk group was defined as CVD risk $\geq 3 \%$.

\section{PLT and WBC count ranges}

Low or high PLT count were set according to age/sex-specific cutoffs. ${ }^{26}$ Low PLT count (thrombocytopenia) was defined as PLT $<156 \times 10^{9} / \mathrm{L}$ or $<140 \times 10^{9} / \mathrm{L}$ for women aged $<64$ years or $>64$ years, respectively; for men, low PLT count was defined as PLT $<141 \times 10^{9} / \mathrm{L}$ or $<122 \times 10^{9} / \mathrm{L}$ for men aged $<64$ years or $>64$ years, respectively.

Cutoffs for high PLT count (thrombocytosis) were set when PLT $>405 \times 10^{9} / \mathrm{L}$ or $>379 \times 10^{9} / \mathrm{L}$ for women aged $<64$ years or $>64$ years, respectively; high PLT count was defined as PLT $>362 \times 10^{9} / \mathrm{L}$ or $>350 \times 10^{9} / \mathrm{L}$ for men aged $<64$ or $>64$ years, respectively.

High (leukocytosis) or low (leukopenia) WBC categories were defined as $\mathrm{WBC}<4 \times 10^{9} / \mathrm{L}$ or $\mathrm{WBC}>10 \times 10^{9} / \mathrm{L}$, respectively. ${ }^{27}$

Neutrophil granulocytes to lymphocyte ratio (NLR) was also evaluated as a marker of inflammation. ${ }^{28}$

\section{Statistical analyses}

Values for continuous variables are means \pm standard deviation (SD) Analysis of variance for continuous or categorical variables was applied to test the associations in Tables 1 and 2. Multivariable linear regression analysis was used for testing the association between PLT or WBC count with adherence to the MDS, FAC, or dietary fiber (considered as continuous independent variables; Table 3).

By using multivariable logistic regression analysis, odds ratios (ORs) with corresponding 95\% confidence intervals (CIs) were calculated to quantify the association between PLT or WBC categories and adherence to a Mediterranean eating pattern (Tables 4 and 5). Four categories of adherence to MD were considered, ranging from low (0-2 points, both for MDS and IMI) to high ( $\geq 7$ points for MDS and $\geq 6$ points for IMI) adherence. An ordinal logistic regression model was used to test whether the impact of diet could be influenced by the cutoff used in defining PLT or WBC groups. Because the assumption of proportional odds failed $(P<.0001$ for each test), we analyzed data by using 2 logistic regression models: one contrasting high PLT (WBC) vs the other group containing normal and low PLT (WBC), and the other contrasting low PLT (WBC) vs the other group containing normal and high PLT (WBC). FAC and dietary fiber were considered either as continuous variables or quartiles.

Both for linear and logistic regression analysis, covariates included in the models were age, sex, BMI, total energy intake, total physical activity, education, smoking, hyperglycemia, high BP, hypercholesterolemia, and 
Table 1. Characteristics of the population sample by PLT or WBC count categories

\begin{tabular}{|c|c|c|c|c|c|c|c|c|c|}
\hline \multirow[b]{2}{*}{ Characteristics } & \multicolumn{5}{|c|}{ PLT count } & \multicolumn{4}{|c|}{ WBC count } \\
\hline & $\begin{array}{c}\text { All } \\
(n=14586)\end{array}$ & $\begin{array}{c}\text { Low } \\
(\mathrm{n}=272,1.9 \%)\end{array}$ & $\begin{array}{c}\text { Normal } \\
(n=13946,95.6 \%)\end{array}$ & $\begin{array}{c}\text { High } \\
(n=368,2.5 \%)\end{array}$ & $P^{*}$ & $\begin{array}{c}\text { Low } \\
(\mathrm{n}=554,3.8 \%)\end{array}$ & $\begin{array}{c}\text { Normal } \\
(n=13690,93.9 \%)\end{array}$ & $\begin{array}{c}\text { High } \\
(\mathrm{n}=342,2.3 \%)\end{array}$ & $P^{\star}$ \\
\hline Age $(y ;$ mean $\pm S D)$ & $54.2(11.5)$ & $55.5(11.7)$ & $54.2(11.5)$ & $53.2(11.8)$ & .020 & $55.2(11.9)$ & $54.2(11.5)$ & $52.9(11.0)$ & .003 \\
\hline Sex (men; n, \%) & $6975(47.8)$ & $135(49.6)$ & $6670(47.8)$ & $170(46.2)$ & .70 & $139(25.1)$ & $6627(48.4)$ & $209(61.1)$ & $<.0001$ \\
\hline $\begin{array}{l}\text { Body mass index } \\
\text { (mean } \pm \text { SD) }\end{array}$ & $27.9(4.7)$ & $28.1(4.7)$ & $27.9(4.7)$ & $27.5(4.9)$ & .15 & $26.0(4.1)$ & $28.0(4.7)$ & $28.6(5.3)$ & $<.0001$ \\
\hline $\begin{array}{r}\text { Physical activity } \\
(\text { mean } \pm \text { SD) }\end{array}$ & $43.1(9.0)$ & $43.9(10.8)$ & $43.1(9.0)$ & $43.3(9.5)$ & .35 & $43.5(9.2)$ & $43.1(9.0)$ & $43.2(9.1)$ & .04 \\
\hline Smokers (n, \%) & $3507(24.0)$ & $68(25.0)$ & 3335 (23.9) & $104(28.3)$ & .07 & $58(10.5)$ & $3232(23.6)$ & $217(63.4)$ & $<.0001$ \\
\hline $\begin{array}{l}\text { Education (secondary } \\
\text { or higher; } \mathrm{n}, \% \text { ) }\end{array}$ & $7212(49.4)$ & $114(41.9)$ & 6919 (49.6) & $179(48.6)$ & .07 & $285(51.4)$ & $6762(49.4)$ & $165(48.2)$ & .08 \\
\hline Hypertension (n, \%) & $8069(55.3)$ & $144(52.9)$ & $7726(55.4)$ & $199(54.0)$ & .41 & $248(44.8)$ & $7630(55.7)$ & $191(55.8)$ & $<.0001$ \\
\hline Diabetes (n, \%) & $1196(8.2)$ & $28(10.3)$ & $1144(8.2)$ & $24(6.5)$ & .24 & $18(3.2)$ & $1128(8.3)$ & $50(14.6)$ & $<.0001$ \\
\hline $\begin{array}{l}\text { Hypercholesterolemia } \\
(\mathrm{n}, \%)\end{array}$ & $4746(32.5)$ & $50(18.4)$ & 4578 (32.8) & $118(32.1)$ & $<.0001$ & $164(29.6)$ & $4464(32.6)$ & $118(34.5)$ & .02 \\
\hline CRP levels (mg/dL) $\dagger$ & $1.42(0.73-2.60)$ & $1.43(0.61-2.35)$ & $1.41(0.73-2.59)$ & $1.65(0.76-3.38)$ & .0004 & $0.87(0.48-1.78)$ & $1.43(0.74-2.59)$ & $2.64(0.37-5.14)$ & $<.0001$ \\
\hline
\end{tabular}

CRP. Further adjustments for FAC and dietary fiber were considered for testing the role of these compounds in explaining the association among PLTs, WBCs, and the MD.

Data were analyzed using SAS/STAT software version 9.1 .3 of the SAS System for Windows 2009 (SAS Institute, Cary, NC).

\section{Results}

Table 1 shows the main characteristics of the population sample according to levels of PLT or WBC count. Subjects in the high-PLT

Table 2. Mean PLT and WBC count according to categories of adherence to the MD and FAC or dietary fiber quartiles

\begin{tabular}{|c|c|c|c|}
\hline & n (\%) & PLT count & WBC count \\
\hline \multicolumn{4}{|l|}{ MDS } \\
\hline Low (0-2) & $1866(12.8)$ & $252.8(64.2)$ & $6.31(1.60)$ \\
\hline Low-medium (3-4) & 5789 (39.7) & $250.3(63.3)$ & $6.24(1.66)$ \\
\hline Medium-high (5-6) & 5381 (36.9) & $249.4(61.5)$ & $6.20(1.57)$ \\
\hline High (7-9) & $1550(10.6)$ & $246.0(57.2)$ & $6.17(2.19)$ \\
\hline$P$ for trend & & .0013 & .004 \\
\hline \multicolumn{4}{|l|}{ IMI } \\
\hline Low (0-2) & $3128(21.4)$ & $252.0(62.4)$ & $6.33(1.72)$ \\
\hline Low-medium (3) & 2995 (20.5) & $252.1(64.4)$ & $6.31(1.97)$ \\
\hline Medium-high (4-5) & $5584(38.3)$ & $249.0(62.0)$ & $6.18(1.55)$ \\
\hline High $(\geq 6)$ & 2879 (19.7) & $246.8(59.9)$ & $6.15(1.56)$ \\
\hline$P$ for trend & & .0001 & $<.0001$ \\
\hline \multicolumn{4}{|l|}{ FAC quartiles } \\
\hline First & 3619 (24.8) & $250.1(63.5)$ & $6.31(1.74)$ \\
\hline Second & $3674(25.1)$ & $250.8(64.1)$ & $6.24(1.55)$ \\
\hline Third & 3631 (24.9) & $251.3(60.6)$ & $6.21(1.56)$ \\
\hline Fourth & $3662(25.1)$ & $247.1(60.5)$ & $6.15(1.86)$ \\
\hline$P$ for trend & & 054 & $<.0001$ \\
\hline \multicolumn{4}{|l|}{ Dietary fiber quartiles } \\
\hline First & $3646(25.0)$ & $250.6(64.6)$ & $6.35(1.65)$ \\
\hline Second & $3647(25.0)$ & $251.3(62.1)$ & $6.21(1.64)$ \\
\hline Third & $3647(25.0)$ & $249.6(62.0)$ & $6.18(1.57)$ \\
\hline Fourth & $3646(25.0)$ & $247.9(59.9)$ & $6.19(1.86)$ \\
\hline$P$ for trend & & .03 & $<.0001$ \\
\hline
\end{tabular}

PLT and WBC counts are reported as mean ( \pm SD). Means and $P$ for trend value adjusted for age and sex. All the analyses with PLTs were further controlled for hematocrit. group were younger and reported higher prevalence of hypercholesterolemia and increased CRP levels compared with those in the normal- or low-PLT count categories. Individuals in the high-WBC category were mainly men, younger, smokers, had a higher BMI and higher CRP levels, and showed a higher prevalence of high BP, hyperglycemia, and hypercholesterolemia (all $P<.05$ ). Both blood cell counts decreased by increasing levels of adherence to a Mediterranean eating pattern (Table 2). Mean PLT or WBC count slightly decreased according to quartiles of $\mathrm{FAC}(P=.05$ and $P<.0001$ for PLTs and WBCs, respectively; Table 2$)$ and to quartiles of dietary fiber intake $(P=.03$ and $P<.0001$ for PLT and WBC count, respectively; Table 2 ). PLT count was inversely associated either with MDS or IMI scores (multivariable adjusted model 2: $\beta=-1.21$; $P<.0001$ and $\beta=-1.28 ; P<.0001$, respectively; Table 3). Similarly, WBC count was inversely linked to an $\mathrm{MD}(\beta=-0.022$ and $P=.008 ; \beta=-0.039$ and $P<.0001$ for MDS and IMI, respectively; Table 3). Further adjustment for CRP did modify the association for PLT or WBC count (multivariable model 3; Table 3).

Additional adjustments for FAC and fiber intake simultaneously were performed in order to account for the observed associations between PLTs or WBCs and adherence to the MD. The inclusion of these 2 dietary components in the multivariable models slightly reduced the association between PLT count and adherence to the MD (Table 3), whereas the relationship between WBC and MDS was reduced and no longer statistically significant (Table 3 ). On the contrary, further adjustment for the $\omega-6$ (linoleic acid) to $\omega-3$ (linolenic acid) fatty acids ratio did not modify the association of adherence to the MD with either PLT or WBC count (data not shown).

Multivariable logistic regression analysis was used to test the odds of being in the low or high category of WBC or PLT counts according to levels of adherence to a Mediterranean dietary pattern. Subjects with very high adherence to the MD reported significantly lower odds of being in the high-PLT count group compared with individuals with poor adherence $(\mathrm{OR}=0.50 ; 95 \% \mathrm{CI}$ : $0.31-0.80$ and $\mathrm{OR}=0.73$; 95\% CI: $0.52-1.02$ for MDS and IMI, respectively; Table 4). The associations were partially explained by FAC and dietary fiber intake when the MD was measured according to IMI, because the strength of the association was reduced or was no longer statistically significant.

No significant relationship was found for the odds of being in the low-PLT-count groups (Table 4). 
Table 3. Multivariable linear regression analysis regarding the association between PLT or WBC and the MD and further adjusted for FAC or dietary fiber intake

\begin{tabular}{|c|c|c|c|c|c|c|c|c|}
\hline \multirow[b]{2}{*}{ Count } & \multicolumn{2}{|l|}{ Model 1} & \multicolumn{2}{|l|}{ Model 2} & \multicolumn{2}{|l|}{ Model 3} & \multicolumn{2}{|l|}{ Model 4} \\
\hline & $\beta(95 \% \mathrm{Cl})$ & $P$ & $\beta(95 \% \mathrm{Cl})$ & $P$ & $\beta(95 \% \mathrm{Cl})$ & $P$ & $\beta(95 \% \mathrm{Cl})$ & $P$ \\
\hline \multicolumn{9}{|l|}{ PLT } \\
\hline MDS & $-1.05(-1.65$ to -0.46$)$ & .0005 & $-1.21(-1.81$ to -0.61$)$ & $<.0001$ & $-1.15(-1.75$ to -0.55$)$ & .0002 & $-0.85(-1.56$ to -0.135 & .020 \\
\hline IMI & $-1.21-1.76$ to -0.66 ) & $<.0001$ & $-1.28(-1.83$ to -0.72$)$ & $<.0001$ & $-1.18(-1.73$ to -0.62$)$ & $<.0001$ & $-0.98(-1.66$ to -0.29$)$ & .005 \\
\hline \multicolumn{9}{|l|}{ WBC } \\
\hline MDS & $-0.024(-0.04$ to -0.01$)$ & .004 & $-0.022(-0.04$ to -0.01$)$ & .008 & $-0.019(-0.03$ to -0.003$)$ & .019 & $-0.009(-0.03$ to 0.01$)$ & .33 \\
\hline IMI & $-0.042(-0.06$ to -0.03$)$ & $<.0001$ & $-0.039(-0.05$ to -0.02$)$ & $<.0001$ & $-0.033(-0.05$ to -0.02$)$ & $<.0001$ & $-0.033(-0.05$ to -0.01$)$ & .0003 \\
\hline
\end{tabular}

Model 1 included age and sex. Model 2 included age, sex, energy intake, BMI, physical activity, smoking, education, hypertension, diabetes, and hypercholesterolemia. Model 3 as in model 2 but further adjusted for CRP. Model 4 as in model 3 but further adjusted for FAC and dietary fiber intake. All the analyses with PLTs were furthe controlled for hematocrit.

Concerning WBC count, a very good adherence to the MD was significantly associated with an increased chance of being in the bottom-WBC-count group (Table 5), and the association was fairly well explained by FAC and dietary fiber intake, especially when adherence to the MD was measured by the MDS (Table 5). No significant relationship was found regarding the association between adherence to the MD and the high-WBC group.

NLR was significantly and inversely associated with adherence to the MD $(P=.0010$ and $P<.0001$ for MDS and IMI, respectively). In addition, leukocyte subgroup analysis showed an inverse relationship between adherence to the MD and either granulocyte $(P=.17$ and $P=.0001$ for MDS and IMI respectively) or monocyte $(P<.0001$ for both MDS and IMI, respectively) count and a positive link with lymphocyte count $(P<.0001$ for both dietary scores).

The association between WBC count and MDS (but not IMI) disappeared when further adjusted for PLT count $(\beta=-0.009$ and $P=.22$ in the multivariate model including CRP). On the contrary, the association between PLT count and both dietary scores remained significant after further controlling for WBC count.
Mean PLT count increased with increasing predicted CVD risk in men (low CVD risk: 236.5 \pm 54.7, medium CVD risk: $239.6 \pm 57.1$, and high CVD risk: $247.1 \pm 58.7 ; P$ for trend $=.027$ in multivariable analysis of variance). In women, we failed to observe any difference in PLT count within the predicted CVD risk groups.

Regarding WBC, we found a clear association with estimated CVD risk in men (low CVD risk: $5.88 \pm 1.43$, medium CVD risk: $6.79 \pm 2.02$, and high CVD risk: $7.59 \pm 1.71 ; P$ for trend $<.0001$ in multivariable analysis of variance). The same trend was observed for women $(P<.0001)$

\section{Discussion}

Recent studies have suggested a significant relationship between the type of diet and chronic low-grade inflammation, an underlying pathophysiological mechanism linking behavioral factors and oxidative stress to the risk of developing chronic disease. ${ }^{29}$ Increased WBC

Table 4. Odds of being in the high- or low-PLT category according to adherence to the MD and further adjusted for FAC or dietary fiber intake

\begin{tabular}{|c|c|c|c|c|c|c|}
\hline \multirow[b]{2}{*}{ Category } & \multicolumn{2}{|c|}{ Model 2} & \multicolumn{2}{|c|}{ Model 3} & \multicolumn{2}{|c|}{ Model 4} \\
\hline & ORs $(95 \% \mathrm{Cl})$ & $P^{*}$ & ORs $(95 \% \mathrm{Cl})$ & $P^{*}$ & ORs $(95 \% \mathrm{Cl})$ & $P^{*}$ \\
\hline \multicolumn{7}{|l|}{ High PLT $(n=368)$} \\
\hline MDS & & .0013 & & .0016 & & .056 \\
\hline Low (0-2) & 1 & & 1 & & 1 & \\
\hline Low-medium (3-4) & $0.79(0.59-1.07)$ & & $0.80(0.59-1.09)$ & & $0.85(0.62-1.16)$ & \\
\hline Medium-high (5-6) & $0.67(0.49-0.92)$ & & $0.68(0.50-0.94)$ & & $0.67(0.54-1.09)$ & \\
\hline High $(7-9)$ & $0.50(0.31-0.80)$ & & $0.50(0.31-0.81)$ & & $0.60(0.36-1.02)$ & \\
\hline IMI & & .048 & & .064 & & .64 \\
\hline Low (0-2) & 1 & & 1 & & 1 & \\
\hline Low-medium (3) & $0.99(0.74-1.36)$ & & $1.01(0.74-1.37)$ & & $1.05(0.77-1.43)$ & \\
\hline Medium-high (4-5) & $0.87(0.66-1.14)$ & & $0.88(0.67-1.16)$ & & $0.97(0.72-1.31)$ & \\
\hline High $(\geq 6)$ & $0.73(0.52-1.02)$ & & $0.74(0.53-1.04)$ & & $0.92(0.61-1.36)$ & \\
\hline \multicolumn{7}{|l|}{ Low PLT $(n=272)$} \\
\hline MDS & & .31 & & .33 & & .84 \\
\hline Low $(0-2)$ & 1 & & 1 & & 1 & \\
\hline Low-medium (3-4) & $0.98(0.66-1.45)$ & & $0.97(0.65-1.44)$ & & $0.92(0.61-1.38)$ & \\
\hline Medium-high (5-6) & $1.03(0.69-1.55)$ & & $1.02(0.69-1.53)$ & & $0.91(0.59-1.41)$ & \\
\hline High (7-9) & $1.28(0.78-2.10)$ & & $1.27(0.78-2.08)$ & & $1.08(0.62-1.87)$ & \\
\hline IMI & & .09 & & .10 & & .39 \\
\hline Low (0-2) & 1 & & 1 & & 1 & \\
\hline Low-medium (3) & $1.27(0.86-1.87)$ & & $1.25(0.85-1.85)$ & & $1.22(0.82-1.81)$ & \\
\hline Medium-high (4-5) & $1.24(0.88-1.77)$ & & $1.23(0.87-1.75)$ & & $1.15(0.79-1.68)$ & \\
\hline High $(\geq 6)$ & $1.45(0.98-2.14)$ & & $1.43(0.96-2.11)$ & & $1.27(0.80-2.01)$ & \\
\hline
\end{tabular}

Model 2 included age, sex, energy intake, BMI, physical activity, smoking, education, hypertension, diabetes, and hypercholesterolemia. Model 3 as in model 2 but further adjusted for CRP. Model 4 as in model 3 but further adjusted for FAC and dietary fiber intake.

${ }^{\star} P$ for trend value. 
Table 5. Odds of being in the high- or low-WBC category according to adherence to the MD and further adjusted for FAC or dietary fiber intake

\begin{tabular}{|c|c|c|c|c|c|c|}
\hline \multirow[b]{2}{*}{ Category } & \multicolumn{2}{|c|}{ Model 2} & \multicolumn{2}{|c|}{ Model 3} & \multicolumn{2}{|c|}{ Model 4} \\
\hline & ORs $(95 \% \mathrm{Cl})$ & $P^{*}$ & ORs $(95 \% \mathrm{Cl})$ & $P^{*}$ & ORs $(95 \% \mathrm{Cl})$ & $\boldsymbol{P}^{\star}$ \\
\hline \multicolumn{7}{|l|}{ High WBC $(n=342)$} \\
\hline MDS & & .18 & & .26 & & .70 \\
\hline Low (0-2) & 1 & & 1 & & 1 & \\
\hline Low-medium (3-4) & $1.05(0.74-1.48)$ & & $1.05(0.74-1.50)$ & & $1.10(0.77-1.57)$ & \\
\hline Medium-high (5-6) & $0.90(0.63-1.29)$ & & $0.94(0.66-1.35)$ & & $1.03(0.69-1.52)$ & \\
\hline High (7-9) & $0.80(0.49-1.30)$ & & $0.80(0.49-1.31)$ & & $0.92(0.53-1.59)$ & \\
\hline IMI & & .12 & & .24 & & .69 \\
\hline Low $(0-2)$ & 1 & & 1 & & 1 & \\
\hline Low-medium (3) & $0.87(0.63-1.19)$ & & $0.92(0.67-1.27)$ & & $0.95(0.69-1.32)$ & \\
\hline Medium-high (4-5) & $0.62(0.47-0.84)$ & & $0.64(0.48-0.87)$ & & $0.70(0.51-0.96)$ & \\
\hline High $(\geq 6)$ & $0.92(0.67-1.27)$ & & $0.99(0.71-1.38)$ & & $1.18(0.79-1.75)$ & \\
\hline \multicolumn{7}{|l|}{ Low WBC $(n=554)$} \\
\hline MDS & & .078 & & .093 & & .40 \\
\hline Low (0-2) & 1 & & 1 & & 1 & \\
\hline Low-medium (3-4) & $1.24(0.92-1.66)$ & & $1.21(0.90-1.63)$ & & $1.17(0.87-1.58)$ & \\
\hline Medium-high (5-6) & $1.41(1.05-1.90)$ & & $1.37(1.02-1.86)$ & & $1.28(0.92-1.76)$ & \\
\hline High (7-9) & $1.26(0.85-1.85)$ & & $1.25(0.84-1.84)$ & & $1.11(0.72-1.71)$ & \\
\hline IMI & & .0031 & & .0052 & & .031 \\
\hline Low $(0-2)$ & 1 & & 1 & & 1 & \\
\hline Low-medium (3) & $1.01(0.76-1.35)$ & & $1.00(0.75-1.34)$ & & $1.00(0.74-1.33)$ & \\
\hline Medium-high (4-5) & $1.30(1.01-1.66)$ & & $1.27(0.99-1.63)$ & & $1.25(0.96-1.63)$ & \\
\hline High $(\geq 6)$ & $1.41(1.07-1.86)$ & & $1.39(1.05-1.83)$ & & $1.34(0.97-1.87)$ & \\
\hline
\end{tabular}

Model 2 included age, sex, energy intake, BMI, physical activity, smoking, education, hypertension, diabetes, and hypercholesterolemia. Model 3 as in model 2 but further adjusted for CRP. Model 4 as in model 3 but further adjusted for FAC and dietary fiber intake.

${ }^{\star} P$ for trend value.

count is widely recognized as a reliable biomarker of inflammation, ${ }^{30}$ but PLTs have also been ascribed a role in inflammation due to the production and release of prostaglandins and other substances causing either vasodilation or vasoconstriction. ${ }^{31-34}$

Our study has investigated whether the adherence to a Mediterranean-style diet is a determinant of PLT or WBC count in a healthy population. Our results show that a greater adherence to the MD, measured by 2 widely used dietary scores, is significantly associated with a reduction in either PLT or WBC count in multivariable models controlling for many possible confounders. In addition to lifestyle covariates, we considered a model further adjusted for CRP. The latter had been previously shown to be inversely associated with a Mediterranean eating pattern ${ }^{35}$ and directly linked both to PLT and WBC count. ${ }^{27}$ The link between PLTs and inflammation is also supported by our present findings due to the direct association of PLTs with CRP, which is a recognized inflammatory marker. Yet, we exclude a role of CRP in explaining the association of PLT or WBC with a Mediterranean-type diet because further controlling for CRP had a minimal impact on our findings.

Similarly, thrombopoietin levels are reportedly increased in inflammatory conditions, and this may result in higher PLT counts. ${ }^{36}$ Lack of data on thrombopoietin levels in our population sample did not allow us to test for the possible involvement of this protein in the association between PLT count and an MD.

The present study refers to subjects apparently free from clinically overt chronic disease and major hematologic pathologies. Thus, the differences observed among categories of PLT or WBC counts indicate substantial changes within normal ranges of variability in a healthy general population. Individuals with high adherence to the MD reported significantly reduced odds of being in the high-PLT group compared with those with poor adherence and an indicative trend in being in the lowest PLT group. Similarly, top adherence to MD was linked to increased odds of being in the bottom WBC count group.
WBC subgroup analyses revealed an inverse link between greater adherence to the MD and neutrophils or monocytes, which are the first WBCs involved in the inflammatory response. ${ }^{37}$ Conversely, a positive association was found with lymphocytes, which are involved in the immune reactivity and have been previously shown to be positively stimulated by some dietary components such as antioxidants. ${ }^{38}$ Moreover, we found an inverse association between the MD and the NLR ratio, which has been recently reported as a prognostic marker for $\mathrm{CVD},{ }^{28}$ further confirming that this dietary pattern is mainly effective on the inflammatory components of WBCs. The relationship between WBC count and adherence to the MD (when measured by the MDS score) disappeared after further controlling for PLT, suggesting the important assumption that the observed associations between a Mediterranean eating pattern and WBC and PLT counts are mainly ascribed to the PLT count.

These observations suggest a positive effect of a Mediterranean eating pattern in reducing either PLT or WBC count and possibly the extent of chronic low-grade inflammation. These are in agreement with an intervention study in healthy Swedish subjects reporting that a supplementation of a kind of MD significantly reduced the number of both PLTs and WBCs. ${ }^{39}$

Further analyses were performed to find possible variables accounting for the observed associations. In particular, we tested the role played by FAC, dietary fiber, and PUFA intake as possible mediators. Both dietary antioxidant content and fiber partially accounted for the association between adherence to the MD and WBC count, suggesting a role of these healthy food components in the effect of the MD. Antioxidants and fiber intake also reduced the strength of the association between PLTs and the MD, leading to similar conclusions. The ratio of dietary $\omega-6$ (linoleic acid) to $\omega-3$ fatty acids (linolenic acid), whose lower value is more desirable in reducing the risk of major chronic diseases, ${ }^{40}$ was significantly associated with WBCs but did not account for the relationship between WBCs and the MD. 
The biological plausibility of the observed associations may rely on the close relationship between oxidative stress and chronic low-grade inflammation (eg, increased CRP, WBCs, and PLTs). ${ }^{41}$ Considering that the high antioxidant and fiber content of the MD has been linked to reduced oxidative stress, ${ }^{42,43}$ it is likely that the antioxidant properties of the MD may contribute to the reduction of subclinical inflammation by reducing the number of leukocytes and PLTs. It has been suggested that the change in the hematology pattern (lower inflammatory blood cells count) observed after supplementation of a Mediterranean-type diet to Swedish subjects could be linked both to a lower inflammatory activity or to a consistent decrease in vasoregulation and in the vascular endothelial growth factor concentrations requiring reduced endothelial cell repair processes. ${ }^{43}$ Previous studies have shown that antioxidants are related to the inhibition of vascular endothelial growth factor release $^{44}$ and to lower inflammation. ${ }^{11}$ This may explain a possible role of these healthy compounds in modulating the number of PLTs or WBCs in response to a low-grade chronic inflammatory status.

However, the dietary antioxidant and fiber content only partially explains the observed associations. This leads us to confirm the assumption that the diet as a whole, not a single food item, is responsible for the beneficial health outcomes reportedly documented. ${ }^{45}$

To our knowledge, this is the first large epidemiological study considering the traditional MD as a possible determinant of the number of PLT and WBC in an adult healthy population.

The observed association between higher adherence to a Mediterranean pattern and lower PLT or WBC counts suggests that this dietary pattern may contribute to reducing 2 important cellular biomarkers of chronic low-grade inflammation. This beneficial effect can be partly ascribed to its pivotal components as food antioxidants and dietary fiber.

\section{Limitations of this study}

A major limitation of this study is its cross-sectional nature, which cannot allow the inference of possible causality. Caution is also needed in extending the results presented here to larger population contexts, because data were collected in a region located between central and southern Italy, Mediterranean by tradition and culture. ${ }^{15}$ Yet, the main characteristics of our population sample are comparable to those of the Italian Cardiovascular Epidemiological Observatory ${ }^{46}$ : our sample could therefore be considered representative at least of the Italian population. ${ }^{26}$

The possibility of residual confounding cannot be entirely excluded, although our analyses have been adjusted for a very large panel of potential confounders.

In addition, we cannot estimate the real improvement, in terms of reduction of the risk of major clinical outcomes, that could be ascribed to the relatively small difference in mean PLTs observed between the lowest and highest group of adherence to the MD.

However, we should recognize that, at least in men, the changes in PLT count within the low and high categories of adherence to an MD are comparable to those observed within the low and high predicted cardiovascular risk groups.

Because the MD has been related to reduced mortality, ${ }^{7}$ we might speculate that this effect could be partly explained by the association of this dietary pattern with lower PLT and WBC counts. However, we cannot exclude that lower PLTs and leukocytes could simply be a marker of adherence to the MD without any clinical significance.
Future studies should estimate to what extent a reduced number of PLTs and/or WBCs, within normal-range values, may contribute to the lowered risk of thrombosis (PLT)- or inflammation (WBC)related diseases that is associated with high adherence to the MD. ${ }^{7}$

\section{Acknowledgments}

The enrollment phase of the Moli-sani Project was performed at the Research Laboratories, Catholic University, Campobasso, Italy. The Moli-sani research group would like to thank Drs Vittorio Krogh and Sabina Sieri from Istituto Nazionale dei Tumori, Milan, Italy, for their contribution to dietary-questionnaire analysis and interpretation.

The enrollment phase of the Moli-sani Project was supported by unrestricted research grants from Pfizer Foundation (Rome, Italy) and the Italian Ministry of University and Research (Rome, Italy), Programma Triennale di Ricerca, Decreto no. 1588. The Pfizer Foundation and the Italian Ministry of University and Research had no role in study design, collection, analysis, and interpretation of data; in the writing of the report; and in the decision to submit the article for publication. All authors were and are independent of funders.

\section{Authorship}

Contribution: M.B. and C.C. designed the research; S.C., A. De Curtis, and M.P. managed data collection; M.B. and A. Di Castelnuovo analyzed the data; M.B. wrote the paper; L.I., M.B.D., and G.d.G. originally inspired the research, obtained the financial support, and critically reviewed the manuscript; and all authors had full access to all of the data in the study and take responsibility for the integrity of the data and the accuracy of the data analysis.

Conflict-of-interest disclosure: The authors declare no competing financial interests.

Correspondence: Licia Iacoviello, Laboratory of Molecular and Nutritional Epidemiology, Department of Epidemiology and Prevention, IRCCS Istituto Neurologico Mediterraneo Neuromed, Via dell'Elettronica, 86077 Pozzilli (Isernia), Italy; e-mail: licia. iacoviello@neuromed.it.

\section{Appendix}

The Moli-sani Project Investigators are - Steering Committee: Licia Iacoviello (Chairperson) (Neuromed, Pozzilli, Italy), Maria Benedetta Donati and Giovanni de Gaetano (Neuromed, Pozzilli, Italy), and Simona Giampaoli (Istituto Superiore di Sanità, Roma, Italy). Safety and Data Monitoring Committee: Jos Vermylen (Chairman) (Catholic Univesity, Leuven, Belgio), Ignacio De Paula Carrasco (Accademia Pontificia Pro Vita, Roma, Italy), Fabrizio Oleari (Istituto Superiore di Sanità, Roma, Italy), and Antonio Spagnuolo (Catholic University, Roma, Italy). Event Adjudicating Committee: Deodato Assanelli (Brescia, Italy), Vincenzo Centritto (Campobasso, Italy), Paola Muti (Hamilton, ON, Canada), Holger Schünemann (Hamilton, ON, Canada), Pasquale Spagnuolo and Dante Staniscia (Termoli, Italy). Scientific and Organizing Secretariat: Francesco Zito (Coordinator), Americo Bonanni, Chiara 
Cerletti, Amalia De Curtis, Augusto Di Castelnuovo, Licia Iacoviello, Roberto Lorenzet, Antonio Mascioli, Marco Olivieri and Domenico Rotilio. Data management and analysis: Augusto Di Castelnuovo (Coordinator), Marialaura Bonaccio, Simona Costanzo and Francesco Gianfagna. Informatics: Marco Olivieri (Coordinator), Maurizio Giacci, Antonella Padulo and Dario Petraroia Biobank and biochemical analyses: Amalia De Curtis (Coordinator), Federico Marracino, Maria Spinelli, Christian Silvestri. Communication and Press Office: Americo Bonanni (Coordinator), Marialaura Bonaccio and Francesca De Lucia. Moli-family Project: Francesco Gianfagna and Branislav Vohnout. Recruitment staff: Franco Zito (General Coordinator); Secretariat: Mariarosaria Persichillo (Coordinator), Angelita Verna, Maura Di Lillo, and Irene Di Stefano; Blood sample: Agostino Pannichella, Antonio Rinaldo Vizzarri,
Branislav Vohnout, and Agnieszka Pampuch; Spirometry: Antonella Arcari (Coordinator), Daniela Barbato, Francesca Bracone, Simona Costanzo, Carmine Di Giorgio, Sara Magnacca, Simona Panebianco, Antonello Chiovitti, Federico Marracino, Sergio Caccamo, and Vanesa Caruso; Electrocardiograms: Livia Rago (Coordinator), Daniela Cugino, Francesco Zito, Francesco Gianfagna, Alessandra Ferri, Concetta Castaldi, Marcella Mignogna and Tomasz Guszcz; Questionnaires: Romina di Giuseppe (Coordinator), Paola Barisciano, Lorena Buonaccorsi, Floriana Centritto, Antonella Cutrone, Francesca De Lucia, Francesca Fanelli, Iolanda Santimone, Anna Sciarretta, Maura Di Lillo, Isabella Sorella, Irene Di Stefano, Emanuela Plescia, Alessandra Molinaro and Christiana Cavone Call Center: Giovanna Galuppo, Maura Di Lillo, Concetta Castaldi, Dolores D'Angelo and Rosanna Ramacciato.

\section{References}

1. Thaulow E, Erikssen J, Sandvik L, Stormorken $\mathrm{H}$, Cohn PF. Blood platelet count and function are related to total and cardiovascular death in apparently healthy men. Circulation. 1991;84 (2):613-617.

2. van der Bom JG, Heckbert SR, Lumley T, et al. Platelet count and the risk for thrombosis and death in the elderly. J Thromb Haemost. 2009; 7(3):399-405

3. Campbell PJ, MacLean C, Beer PA, et al. Correlation of blood counts with vascular complications in essential thrombocythemia analysis of the prospective PT1 cohort. Blood. 2012;120(7):1409-1411.

4. Coller BS. Leukocytosis and ischemic vascular disease morbidity and mortality: is it time to intervene? Arterioscler Thromb Vasc Biol. 2005; 25(4):658-670.

5. Rienstra M, Sun JX, Magnani JW, et al. White blood cell count and risk of incident atrial fibrillation (from the Framingham Heart Study) Am J Cardiol. 2012;109(4):533-537.

6. Centritto F, lacoviello L, di Giuseppe R, et al; Moli-sani Investigators. Dietary patterns, cardiovascular risk factors and C-reactive protein in a healthy Italian population. Nutr Metab Cardiovasc Dis. 2009;19(10):697-706.

7. Sofi F, Abbate R, Gensini GF, Casini A. Accruing evidence on benefits of adherence to the Mediterranean diet on health: an updated systematic review and meta-analysis. Am J Clin Nutr. 2010;92(5):1189-1196.

8. Bonaccio M, lacoviello L, de Gaetano G; MoliSani Investigators. The Mediterranean diet: the reasons for a success. Thromb Res. 2012;129(3): 401-404.

9. Bonaccio M, Di Castelnuovo A, Bonanni A, et al. Adherence to a Mediterranean diet is associated with a better health-related quality of life: a possible role of high dietary antioxidant content. BMJ Open. 2013;3(8):e003003.

10. Giacosa A, Barale R, Bavaresco L, et al. Cancer prevention in Europe: the Mediterranean diet as a protective choice. Eur J Cancer Prev. 2013; 22(1):90-95.

11. Scoditti E, Calabriso N, Massaro M, et al. Mediterranean diet polyphenols reduce inflammatory angiogenesis through MMP-9 and inflammatory angiogenesis through MMP-9 and COX-2 inhibition in human vascular endothe
cells: a potentially protective mechanism in cells: a potentially protective mechanism in
atherosclerotic vascular disease and cancer. Arch Biochem Biophys. 2012;527(2):81-89.

12. Satija A, Hu FB. Cardiovascular benefits of dietary fiber. Curr Atheroscler Rep. 2012;14(6):505-514.

13. Crowe FL, Key TJ, Appleby PN, et al. Dietary fibre intake and ischaemic heart disease mortality: the European Prospective Investigation into Cancer and Nutrition-Heart study. Eur J Clin Nutr. 2012; 66(8):950-956

14. Chowdhury R, Stevens S, Gorman D, et al. Association between fish consumption, long chain omega 3 fatty acids, and risk of cerebrovascula disease: systematic review and meta-analysis. BMJ. 2012;345:e6698.

15. lacoviello $L$, Bonanni $A$, Costanzo $S$, et al. The Moli-sani Project, a randomized, prospective cohort study in the Molise region in Italy: design rationale and objectives. Int J Public Health. 2007; 4(2):110-118.

16. Pala V, Sieri S, Palli D, et al. Diet in the Italian EPIC cohorts: presentation of data and methodological issues. Tumori. 2003;89(6): 594-607.

17. Pisani $P$, Faggiano $F$, Krogh $V$, Palli $D$, Vineis $P$, Berrino $F$. Relative validity and reproducibility of a food frequency dietary questionnaire for use in the Italian EPIC centres. Int J Epidemiol. 1997; 26(suppl 1):S152-S160.

18. Trichopoulou A, Costacou T, Bamia C Trichopoulos D. Adherence to a Mediterranean diet and survival in a Greek population. $N$ Engl Med. 2003;348(26):2599-2608.

19. Agnoli C, Krogh V, Grioni S, et al. A priori-defined dietary patterns are associated with reduced risk of stroke in a large Italian cohort. J Nutr. 2011; 141(8):1552-1558.

20. Pounis G, Costanzo S, di Giuseppe R, et al. Consumption of healthy foods at different conten of antioxidant vitamins and phytochemicals and of antioxidant vitamins and phytochemicals and metabolic risk factors for cardiovascular disease
in men and women of the Moli-sani study. Eur $J$ in men and women of the Moli-sa
Clin Nutr. 2013;67(2):207-213.

21. Di Castelnuovo A, di Giuseppe R, lacoviello L, de Gaetano G. Consumption of cocoa, tea and coffee and risk of cardiovascular disease. Eur $J$ Intern Med. 2012;23(1):15-25.

22. Janssen I, Katzmarzyk PT, Ross R. Body mass index, waist circumference, and health risk: evidence in support of current National Institutes of Health guidelines. Arch Intern Med. 2002; 162(18):2074-2079.

23. European Society of Hypertension-European Society of Cardiology Guidelines Committee. 2003 European Society of HypertensionEuropean Society of Cardiology guidelines for the management of arterial hypertension the management of arterial hyperten
$J$ Hypertens. 2003;21(6):1011-1053.

24. Nuorti JP, Butler JC, Farley MM, et al; Active Bacterial Core Surveillance Team. Cigarette smoking and invasive pneumococcal disease. N Engl J Med. 2000;342(10):681-689.

25. Ferrario M, Chiodini $P$, Chambless LE, et al; CUORE Project Research Group. Prediction of coronary events in a low incidence population.
Assessing accuracy of the CUORE Cohort Stud prediction equation. Int J Epidemiol. 2005;34(2): 413-421.

26. Biino G, Santimone I, Minelli C, et al. Age- and sex-related variations in platelet count in Italy: a proposal of reference ranges based on 40987 subjects' data. PLOS ONE. 2013;8(1):e54289.

27. Santimone I, Di Castelnuovo A, De Curtis A, et al; MOLI-SANI Project Investigators. White blood cell count, sex and age are major determinants of heterogeneity of platelet indices in an adult general population: results from the MOLI-SAN project. Haematologica. 2011;96(8):1180-1188.

28. Bhat T, Teli S, Rijal J, et al. Neutrophil to lymphocyte ratio and cardiovascular diseases: a review. Expert Rev Cardiovasc Ther. 2013 11(1):55-59.

29. Barbaresko J, Koch M, Schulze MB, Nöthlings $U$. Dietary pattern analysis and biomarkers of lowgrade inflammation: a systematic literature review. Nutr Rev. 2013;71(8):511-527.

30. Danesh J, Whincup P, Walker M, et al. Low grade inflammation and coronary heart disease: prospective study and updated meta-analyses. BMJ. 2000;321(7255):199-204.

31. Weyrich AS, Lindemann S, Zimmerman GA. The evolving role of platelets in inflammation. $J$ Thromb Haemost. 2003;1(9):1897-1905.

32. de Gaetano G, Cerletti C, Nanni-Costa MP, Poggi A. The blood platelet as an inflammatory cell. Eur Respir J Suppl. 1989;6:441s-445s.

33. Gresele P, Falcinelli E, Loffredo F, et al. Platelets release matrix metalloproteinase-2 in the coronary circulation of patients with acute coronary syndromes: possible role in sustained platelet activation. Eur Heart J. 2011;32(3): 316-325.

34. Rondina MT, Weyrich AS, Zimmerman GA. Platelets as cellular effectors of inflammation in vascular diseases. Circ Res. 2013;112(11): 1506-1519.

35. Chrysohoou C, Panagiotakos DB, Pitsavos C Das UN, Stefanadis C. Adherence to the Mediterranean diet attenuates inflammation and coagulation process in healthy adults: The ATTICA Study. J Am Coll Cardiol. 2004;44(1): 152-158.

36. Ceresa IF, Noris P, Ambaglio C, Pecci A, Balduin $\mathrm{CL}$. Thrombopoietin is not uniquely responsible for thrombocytosis in inflammatory disorders. Platelets. 2007;18(8):579-582.

37. Swirski FK, Robbins CS. Neutrophils usher monocytes into sites of inflammation. Circ Res. 2013;112(5):744-745.

38. Chew BP, Park JS. Carotenoid action on the immune response. J Nutr. 2004;134(1): 257S-261S. 
39. Ambring A, Johansson M, Axelsen M, Gan L, Strandvik B, Friberg P. Mediterranean-inspired diet lowers the ratio of serum phospholipid $n-6$ to $n-3$ fatty acids, the number of leukocytes and platelets, and vascular endothelial growth factor in healthy subjects. Am J Clin Nutr. 2006;83(3): 575-581.

40. Simopoulos AP. The importance of the omega-6/ omega-3 fatty acid ratio in cardiovascular disease and other chronic diseases. Exp Biol Med (Maywood). 2008;233(6):674-688 [Maywood]
41. Singh U, Devaraj S, Jialal I. Vitamin E, oxidative stress, and inflammation. Annu Rev Nutr. 2005; 25:151-174.

42. Dai J, Jones DP, Goldberg J, et al. Association between adherence to the Mediterranean diet and oxidative stress. Am J Clin Nutr. 2008;88(5): 1364-1370.

43. Ambring $A$, Friberg $P$, Axelsen $M$, et al. Effects of a Mediterranean-inspired diet on blood lipids. a Mediterranean-inspired diet on blood lipids,
vascular function and oxidative stress in health vascular function and oxidative stress in healthy
subjects. Clin Sci (Lond). 2004;106(5):519-525.
44. Schindler R, Mentlein R. Flavonoids and vitamin E reduce the release of the angiogenic peptide vascular endothelial growth factor from human tumor cells. J Nutr. 2006;136(6):1477-1482.

45. Hu FB. Dietary pattern analysis: a new direction in nutritional epidemiology. Curr Opin Lipidol. 2002; 13(1):3-9.

46. Giampaoli S, Rielli R, Dematté $L$, et al. The Italian observatory of cardiovascular risk: the CUORE project experience [abstract]. Circulation. 2009; project experic
119:e349. 



\section{CHAPTER 5.}

Adherence to the traditional Mediterranean diet and mortality in subjects with diabetes. Prospective results from the Moli-sani study

Bonaccio M, Di Castelnuovo A, Costanzo S, Persichillo M, De Curtis A, Donati MB, de Gaetano $\mathrm{G}$ andIacoviello L, on behalf of the Moli-sani project investigators.

Eur J Prev Cardiol. 2015 Feb 3. pii: 2047487315569409. [Epub ahead of print] 


\title{
Adherence to the traditional Mediterranean diet and mortality in subjects with diabetes. Prospective results from the MOLI-SANI study
}

\author{
European Journal of Preventive \\ Cardiology \\ $0(00) 1-8$ \\ (C) The European Society of \\ Cardiology 2015 \\ Reprints and permissions: \\ sagepub.co.uk/journalsPermissions.nav \\ DOI: $10.1177 / 2047487315569409$ \\ ejpc.sagepub.com \\ (SAGE
}

\author{
Marialaura Bonaccio, Augusto Di Castelnuovo, \\ Simona Costanzo, Mariarosaria Persichillo, Amalia De Curtis, \\ Maria Benedetta Donati, Giovanni de Gaetano and \\ Licia lacoviello on behalf of the MOLI-SANI study \\ Investigators
}

\begin{abstract}
Background: Adherence to the Mediterranean diet is associated with lower mortality in a general population but limited evidence exists on the effect of a Mediterranean diet on mortality in subjects with diabetes. We aim to examine the association between the Mediterranean diet and mortality in diabetic individuals.

Design: Prospective cohort study on 1995 type 2 diabetic subjects recruited within the MOLI-SANI study.

Methods: Food intake was recorded by the European Project Investigation into Cancer and Nutrition food frequency questionnaire. Adherence to the Mediterranean diet was appraised by the Greek Mediterranean diet score. Hazard ratios were calculated using multivariable Cox-proportional hazard models.

Results: During follow-up (median 4.0 years), 109 all-cause including 51 cardiovascular deaths occurred. A 2-unit increase in Mediterranean diet score was associated with $37 \%$ (19\%-51\%) lower overall mortality. Data remained unchanged when restricted to those being on a hypoglycaemic diet or on antidiabetic drug treatment. A similar reduction was observed when cardiovascular mortality only was considered (hazard ratio $=0.66 ; 0.46-0.95$ ). A Mediterranean diet-like pattern, originated from principal factor analysis, indicated a reduced risk of overall death (hazard ratio $=0.8 \mathrm{I}$; 0.62-1.07). The effect of Mediterranean diet score was mainly contributed by moderate alcohol drinking (14.7\% in the reduction of the effect), high intake of cereals $(12.2 \%)$, vegetables $(5.8 \%)$ and reduced consumption of dairy and meat products (13.4\% and $3.4 \%$ respectively).

Conclusions: The traditional Mediterranean diet was associated with reduced risk of both total and cardiovascular mortality in diabetic subjects, independently of the severity of the disease. Major contributions were offered by moderate alcohol intake, high consumption of cereals, fruits and nuts and reduced intake of dairy and meat products.
\end{abstract}

\section{Keywords}

Mediterranean diet, diabetes, overall mortality, cardiovascular mortality

Received 12 July 2014; accepted 6 January 2015

\section{Introduction}

The traditional Mediterranean diet (MD) is an eating pattern typical of the Mediterranean basin and is characterized by a wide consumption of vegetables, cereals, legumes, fish, nuts, olive oil as the main fat source and moderate wine consumption. ${ }^{1,2}$ This diet was reportedly linked to lower risk or mortality for major chronic
Department of Epidemiology and Prevention, IRCCS Istituto Neurologico Mediterraneo NEUROMED, Italy

For a list of MOLI-SANI Study Investigators, see Appendix I in Supplementary Material.

Corresponding author:

Marialaura Bonaccio, Laboratory of Molecular and Nutritional Epidemiology, Department of Epidemiology and Prevention, IRCCS Istituto Neurologico Mediterraneo NEUROMED Via dell'Elettronica, 86077 Pozzilli (Isernia), Italy.

Email: marialaura.bonaccio@neuromed.it 
diseases both in the general population ${ }^{3}$ and in cardiovascular patients, in observational ${ }^{4}$ or intervention studies; ${ }^{5}$ additionally, the MD has been associated with reduced incidence of diabetes. ${ }^{6,7}$ Diabetes affects about 400 million people worldwide ${ }^{8}$ and is estimated to become the seventh leading cause of death in $2030 .^{9}$ Together with other healthy behaviours, following a healthy diet has been assigned a pivotal role in reducing the risk of diabetes. ${ }^{10,11}$ Health advantages of the MD in the prevention of diabetes have been documented in longitudinal $^{6}$ and intervention studies, ${ }^{12}$ but the actual contribution of the traditional MD in reducing mortality in diabetic patients has been poorly explored. So far, there are no controlled clinical trials that specifically established the effect of MD in reducing cardiovascular events or mortality in subjects with diabetes ${ }^{13}$ with the exception of two studies that included a representative diabetic sub-population. ${ }^{14,15}$ Similarly, little is known from observational studies except for one investigation showing that migrants to Australia from Mediterranean countries have lower mortality than do native-born and that frequent consumption of traditional Mediterranean foods is associated with reduced cardiovascular mortality. ${ }^{16,17}$ Scarce evidence exists on the relationship of dietary patterns obtained from a posteriori approaches and mortality ${ }^{16,18}$ in diabetics. Conversely, single food approaches are more numerous and basically addressed advantages of some specific dietary components in reducing the risk of diabetes. ${ }^{19}$

In the present study we examined whether greater adherence to the traditional MD in a Mediterranean region, such as Southern Italy, can be of help in reducing overall and/or cardiovascular mortality in subjects with diabetes. This study also investigated the contribution of single dietary components to the overall effect of the MD in relation to mortality.

\section{Methods}

\section{Study population}

The MOLI-SANI study is a population-based cohort study recruiting 24,325 citizens (men and women aged $\geq 35$ years) of the Molise region, an area placed between Central and Southern Italy, between March 2005 and April 2010, with the purpose of investigating genetic and environmental risk factors in the onset of cardiovascular, cerebrovascular and tumour diseases. ${ }^{20}$

For the present study, subjects with diabetes at time of enrolment were selected for the analysis. Diabetes was defined as use of antidiabetic treatment or blood glucose $\geq 126 \mathrm{mg} / \mathrm{dl}$. Individuals reporting at baseline a personal history of cancer $(3.3 \%)$, those with unreliable dietary or medical questionnaires $(3.9 \%$ and $1 \%$, respectively), subjects lost at follow-up $(n=44)$ or subjects with type 1 diabetes $(n=38)$ were not included in the analysis. The final sample was 1995 patients with type 2 diabetes. Mortality was recorded until December 2011. Overall and cause-specific mortality was assessed by the Italian mortality registry (ReNCaM registry) validated by Italian death certificates (ISTAT form) and coded according to the International Classification of Diseases (ICD-9). A critical evaluation of the diagnosis was performed by analysing hospital medical records for hospital deaths and for other deceased patients if hospitalized during the follow-up.

Cardiovascular deaths were identified when the underlying cause of death had an ICD-9 code of $390-459$ or $745-747$.

The MOLI-SANI study was approved by the Ethics Committee of the Catholic University of Rome, Italy. All participants signed an informed consent.

\section{Dietary information}

Food intake during the year before enrolment was ascertained by the validated Italian version of the 'European project investigation into cancer and nutrition' (EPIC) food frequency questionnaire, ${ }^{21,22}$ which includes 188 food items, later classified into 45 predefined food groups on the basis of similar nutrient characteristics or culinary usage (see Appendix 2 in Supplementary Material).

We evaluated the adherence to the traditional MD by using the Mediterranean Diet Score developed by Trichopoulou et al. $^{23}$ Scoring was calculated in the MOLI-SANI population free from cardiovascular disease, cancer or diabetes and was based on the intake of the following nine items: vegetables; legumes; fruit and nuts; dairy products; cereals; meat and meat products; fish; alcohol; monounsaturated:saturated fats ratio. For most items, consumption above the study median received 1 point; all other intakes received 0 points. For dairy products, meat and meat products, consumption below the median received 1 point. Medians were gender specific. For ethanol, men who consumed $10-50 \mathrm{~g} /$ day and women who consumed 5-25 g/ day received 1 point; otherwise, the score was 0 . The possible scores ranged between 0 and 9, the latter reflecting the maximal adherence to MD.

Food consumption was also evaluated by using principal factor analysis (PFA) conducted on the correlation matrix of 45 food groups. ${ }^{24}$

\section{Anthropometric measurements and assessment of risk factors}

Body mass index was calculated as $\mathrm{kg} / \mathrm{m}^{2}$ and then categorized into three levels as normal $(\leq 25)$, overweight $(>25$ and $<30)$ or obese $(\geq 30)$. Hypertension was 
Table I. Characteristics of the diabetic population at baseline according to adherence to the Mediterranean diet as measured by MD score.

\begin{tabular}{|c|c|c|c|c|c|}
\hline & \multirow[b]{2}{*}{ Whole sample $n=1995$} & \multicolumn{3}{|c|}{ Adherence to the Mediterranean diet (MD score) } & \multirow[b]{2}{*}{$P$ value $^{\mathrm{a}}$} \\
\hline & & Poor $(0-3) n=600$ & Average $(4-5) n=880$ & High $(\geq 6) n=515$ & \\
\hline Age & $62.6(10.2)$ & $63.1(10.3)$ & $62.5(10.1)$ & $62.3(10.3)$ & 0.51 \\
\hline Sex (men) & $1319(66.1)$ & $355(59.2)$ & $601(68.3)$ & $363(70.5)$ & $<.0001$ \\
\hline Education & & & & & 0.048 \\
\hline Secondary school or lower & $1313(65.8)$ & $420(70.0)$ & $578(65.7)$ & $315(61.2)$ & \\
\hline High school or higher & $682(34.2)$ & $180(30.0)$ & $302(34.3)$ & $200(38.8)$ & \\
\hline BMI & & & & & 0.25 \\
\hline Normal $(<25)$ & $210(10.6)$ & $55(9.2)$ & $107(12.2)$ & $48(9.4)$ & \\
\hline Overweight $(\geq 25<30)$ & $749(37.6)$ & $233(38.9)$ & $320(36.4)$ & $196(38.2)$ & \\
\hline Obese $(\geq 30)$ & $1032(51.8)$ & $311(51.9)$ & $452(51.4)$ & $269(52.4)$ & \\
\hline Smoking & & & & & 0.13 \\
\hline Never smokers & 851 (42.7) & $298(49.7)$ & $349(39.7)$ & $204(39.6)$ & \\
\hline Smokers & $384(19.3)$ & $94(15.7)$ & $184(20.9)$ & $106(20.6)$ & \\
\hline Former smokers & $760(38.1)$ & $208(34.7)$ & 347 (39.4) & $205(39.8)$ & \\
\hline Leisure-time physical activity & & & & & $<.0001$ \\
\hline Below median & $1005(50.4)$ & $353(58.8)$ & $426(48.4)$ & $226(43.9)$ & \\
\hline Above median & $990(49.6)$ & $247(4 I .2)$ & $454(51.6)$ & $289(56.1)$ & \\
\hline Hypertension & I 654 (82.9) & $498(83.0)$ & $733(83.3)$ & $423(82.1)$ & 0.61 \\
\hline Hypercholesterolemia & $821(4 I .2)$ & $233(38.8)$ & $374(42.5)$ & $214(4 \mid .6)$ & 0.21 \\
\hline Cardiovascular disease & $258(12.9)$ & $83(13.8)$ & $110(12.5)$ & $65(12.6)$ & 0.25 \\
\hline Blood glucose (mg/dl) & $154.7(45)$ & I53.I (42.2) & I55.5 (46.3) & $155.0(45.9)$ & 0.67 \\
\hline Years from diagnosis of diabetes & & & & & 0.0041 \\
\hline$<5$ & $363(18.2)$ & $89(14.8)$ & $167(19.0)$ & $107(20.8)$ & \\
\hline $5<11.5$ & $312(15.6)$ & $92(15.3)$ & $135(15.3)$ & $85(16.5)$ & \\
\hline$>11.5$ & $332(16.6)$ & $129(21.5)$ & $127(14.4)$ & $76(14.8)$ & \\
\hline Unknown & $988(49.5)$ & $290(48.3)$ & $45 I(51.3)$ & $247(48.0)$ & \\
\hline Energy intake (Kcal/day) & 1971 (636) & $1788(58 \mid)$ & $1996(665)$ & $2143(591)$ & $<.0001$ \\
\hline Hypoglycaemic diet & 761 (38.2) & $233(38.8)$ & $324(36.8)$ & $204(39.6)$ & 0.73 \\
\hline Drug treatment for diabetes & $998(50.0)$ & $313(52.2)$ & $428(48.6)$ & 257 (49.9) & 0.59 \\
\hline Statin use & 361 (18.1) & $102(17.0)$ & $166(18.9)$ & $93(18.1)$ & 0.46 \\
\hline
\end{tabular}

${ }^{\text {a }} P$ value adjusted for sex and age.

Continuous variables (age, blood glucose and energy intake) are expressed as means $\pm \mathrm{SD}$. Categorical variables are expressed as numbers and percentages.

defined as systolic blood pressure (BP) $\geq 140 \mathrm{~mm} \mathrm{Hg}$ and/or diastolic $\mathrm{BP} \geq 90 \mathrm{~mm} \mathrm{Hg}$ or by pharmacological treatment. Hypercholesterolaemia was defined if total cholesterol level was $\geq 240 \mathrm{mg} / \mathrm{dl}$ or by hypolipidaemic treatment. Subjects were classified as never-smokers, current smokers or ex-smokers (quitting from at least 1 year).

Leisure-time physical activity (LTPA) was assessed by a structured questionnaire (questions on sport participation, walking and gardening) and expressed as daily energy expenditure in metabolic equivalent taskhours $(\mathrm{MET} / \mathrm{d})$. LTPA was considered as below $(\leq 2.25)$ or above $(>2.25)$ the median. Educational level was divided into secondary school or lower and high school or higher.

\section{Statistical analyses}

Baseline characteristics were presented as numbers and percentages, or mean values and standard deviation. Analysis of variance for continuous or categorical variables was applied to test the associations in Tables 1 and 2.

Hazard ratios (HR) for overall and cardiovascular/ cerebrovascular mortality for diabetics were calculated using the Cox proportional hazard model with corresponding $95 \%$ confidence intervals $(95 \% \mathrm{CI})$ adjusted 
Table 2. Overall and cardiovascular/cerebrovascular mortality risk in diabetic subjects according to Mediterranean diet (MD).

\begin{tabular}{|c|c|c|c|c|c|}
\hline \multirow[b]{2}{*}{ Number (\%) } & \multicolumn{3}{|c|}{ Adherence to the Mediterranean diet (MD score) } & \multirow[b]{3}{*}{$P$ for trend } & \multirow[b]{3}{*}{$\begin{array}{l}2 \text { point increase in } \\
M D \text { adherence }\end{array}$} \\
\hline & $\begin{array}{l}\text { Poor }(0-3) \\
600(30.1)\end{array}$ & $\begin{array}{l}\text { Average (4-5) } \\
880(44.1)\end{array}$ & $\begin{array}{l}\text { High }(\geq 6) \\
515(25.8)\end{array}$ & & \\
\hline & \multicolumn{3}{|c|}{ Overall mortality $(n=109)$} & & \\
\hline $\begin{array}{l}\text { Number of } \\
\text { deaths (\%) }\end{array}$ & $39(6.5)$ & $51(5.8)$ & $19(3.7)$ & & \\
\hline $\begin{array}{l}\text { Age/adjusted } \\
\text { HR (95\% Cl) }\end{array}$ & $-1-$ & $0.72(0.47-1.10)$ & $0.4 I(0.24-0.72)$ & 0.0015 & $0.62(0.49-0.79)$ \\
\hline \multirow{2}{*}{$\begin{array}{l}\text { Multivariable } \\
\qquad \text { HR }(95 \% \mathrm{Cl})^{\mathrm{a}}\end{array}$} & $-1-$ & $0.71(0.46-1.10)$ & $0.41(0.23-0.73)$ & 0.0023 & $0.63(0.49-0.81)$ \\
\hline & \multicolumn{3}{|c|}{ CVD mortality $(n=5 \mathrm{I})$} & & \\
\hline $\begin{array}{l}\text { Number of } \\
\text { CVD deaths }\end{array}$ & $17(2.8)$ & $24(2.7)$ & $10(1.9)$ & & \\
\hline $\begin{array}{l}\text { Age/adjusted } \\
\text { HR (95\% Cl) }\end{array}$ & $-1-$ & $0.71(0.38-1.35)$ & $0.45(0.21-1.00)$ & 0.048 & $0.67(0.47-0.95)$ \\
\hline $\begin{array}{l}\text { Multivariable } \\
\qquad \text { HR }(95 \% \mathrm{Cl})^{\mathrm{a}}\end{array}$ & $-1-$ & $0.71(0.37-1.38)$ & $0.43(0.19-0.99)$ & 0.046 & $0.66(0.46-0.95)$ \\
\hline
\end{tabular}

CVD: Cardiovascular deaths were identified when the underlying cause of death had an ICD-9 code of 390 -459 or 745-747.

${ }^{a} \mathrm{Hazard}$ ratios (HR) with $95 \%$ confidence intervals $(\mathrm{Cl})$ obtained from a model including age, sex, education, total energy intake, leisure-time physical activity, smoking, years from diagnosis of diabetes, blood glucose and hypercholesterolaemia.

for age, sex, education, energy intake, LTPA, smoking, years from diagnosis of diabetes, blood glucose levels and hypercholesterolaemia.

The MD score was used either as continuous variable (2point increase) or categorized into three levels ranging from poor ( $0-3$ points), average ( $4-5)$ to high $(\geq 6)$ adherence.

PFA was used to identify dietary patterns. ${ }^{23}$ In determining the number of factors to retain in PFA, we used the criteria of an eigenvalue $>1.0$, the scree test and the interpretability of the final solution. ${ }^{24}$ The factors were transformed by the orthogonal varimax rotation, which maintains uncorrelated factors, giving them a simpler structure and a greater interpretability. ${ }^{24}$ The correlations between each extracted factor and foods are called factor loadings; as a rule of thumb, we characterized the factors using the foods with an absolute factor loading greater than 0.15. Each subject received, for each pattern, a factor score, calculated by summing observed intake of the 45 food groups, each weighted by factor loadings. ${ }^{24}$ Analysis for PFA dietary patterns was performed by including the patterns in the model, either as continuous variables or tertiles. This a posteriori approach offers a further evaluation of the eating behaviours and differs from the a priori approach primarily because it is able to catch the real dietary habits of people and accounts for correlations between score components.

The relative importance of each food included in the MD score on mortality was assessed by following the method previously described by Trichopoulou et al. ${ }^{25}$ Briefly, we removed one component at a time alternately from the original score - thus reducing the 10 levels score ( $0-9$ points) to nine levels scores - and estimating the nine mortality ratios associated with a 2-unit increment in the score minus vegetables, score minus legumes score minus fruits and nuts, score minus cereals, score minus fish and seafood, score minus lipid ratio, score minus meat and meat products, score minus dairy products, and score minus ethanol. To assure comparability, we multiplied the logarithm of the estimated nine mortality ratios by $9 / 10$ before exponentiating them (Table 3 ).

The data analysis was generated using SAS/STAT software, Version 9.1.3 of the SAS System for Windows $(2009$. SAS Institute Inc. and SAS are registered trademarks of SAS Institute Inc., Cary, NC, USA.

\section{Results}

During a median of 4.0 years of follow-up (range: 0.14 6.8 years; 8250 person-years), 109 all-cause including 51 cardiovascular/cerebrovascular deaths occurred among 1995 subjects with prevalent diabetes at recruitment.

\section{Association of an a priori score and mortality}

Table 1 shows the main characteristics of the sample at baseline according to adherence to the MD. Subjects with the greatest adherence were mainly men, practised more 
Table 3. Risk of death associated with 2-point increase in the Mediterranean diet score and after alternate subtraction of each of its dietary components.

\begin{tabular}{llcc}
\hline & Risk of death $(95 \% \mathrm{Cl})$ & $P$ value & Reduction in the total effect $(\%)$ \\
\hline Mediterranean diet score (2-point increase) & $0.632(0.494-0.803)$ & 0.0003 & - \\
Food item & $0.686(0.541-0.87 I)$ & 0.0020 & -14.7 \\
Moderate alcohol consumption & $0.681(0.539-0.862)$ & 0.0014 & -13.4 \\
Dairy products (low intake) & $0.677(0.540-0.847)$ & 0.0007 & -12.2 \\
Cereals & $0.653(0.507-0.841)$ & 0.0010 & -5.8 \\
Monounsaturated/saturated ratio & $0.653(0.512-0.834)$ & 0.0006 & -5.8 \\
Vegetables & $0.651(0.519-0.817)$ & 0.0002 & -5.2 \\
Fruits and nuts & $0.650(0.508-0.833)$ & 0.0006 & -5.0 \\
Fish & $0.645(0.510-0.814)$ & 0.0002 & -3.4 \\
Meat and meat products (low intake) & $0.600(0.469-0.767)$ & $<.0001$ & 8.7 \\
Legumes & & & \\
\hline
\end{tabular}

$\mathrm{Cl}$ : confidence intervals.

Hazard ratios from the model controlled for age, sex, education, total energy intake, total physical activity, smoking, years from diagnosis of diabetes, blood glucose and hypercholesterolemia.

physical exercise during leisure time, were more recently diagnosed with diabetes and had a higher energy intake.

Table 2 shows overall and cardiovascular/cerebrovascular mortality ratios according to a 2-unit increment in the MD score and to three categories of adherence to the MD. Increased adherence to the MD by 2 points was associated with $37 \%(95 \%$ CI 19\%-51\%) reduced all-cause mortality rate, in multivariable analysis. Cardiovascular/cerebrovascular deaths were also reduced by $34 \%(5 \%-54 \%)$.

Both all-cause and cardiovascular/cerebrovascular mortality follows a linear trend of reduction according to categories of increasing adherence to a MD diet (Table 2). The inverse relationship between adherence to the MD and risk of overall mortality remained unchanged when analyses were restricted to subjects on drug treatment for diabetes $(n=998 ; \mathrm{HR}=0.60 ; 95 \%$ CI $0.43-0.82$ for a 2-unit increment in MD score) or when further controlled for being on a hypoglycaemic diet $(n=761$; $\mathrm{HR}=0.63 ; 0.49-0.80)$ or statin use $(n=361 ; \mathrm{HR}=0.63$; 95\% CI 0.49-0.81).

\section{Association of an a posteriori score and mortality}

The association between dietary habits and mortality among subjects with diabetes was also tested by a posteriori PFA. Three main factors emerged, in agreement with previous findings in the same population. ${ }^{23}$ The three factors accounted for $13.5 \%$ of the total variation in original food groups. The pattern identified as 'olive oil and vegetables' was characterized by high positive loadings for olive oil, vegetables, legumes, soups, fruits and fish. The pattern named 'pasta and meat' was characterized by high positive loadings for pasta, cooked tomatoes, red meat, animal fats and alcoholic beverages and negative loadings of breakfast cereals and yogurt. The 'eggs and sweets' pattern was characterized by high positive loadings for eggs, margarines, processed meat and sugar and sweets.

Adherence to the so-called 'olive oil and vegetables' pattern was associated with a reduction of $21 \%$ (for 1 SD increase) of all-cause mortality in the age and sex adjusted model $(\mathrm{HR}=0.79 ; 0.63-0.99)$, but the relationship was marginally lost in the fully controlled model $(\mathrm{HR}=0.81 ; 0.62-1.07)$. Consistently, adherence to the 'eggs and sweets' pattern was linked to an increased risk of death, although not significant $(\mathrm{HR}=1.34 ; 0.98-1.83)$. The 'pasta and meat' pattern was not associated with reduced risk of mortality $(\mathrm{HR}=0.96 ; 0.69-1.32)$.

\section{Contribution to the association of single food items}

Table 3 shows the modification of the overall mortality ratio when each of the nine components of the score was alternatively removed. In this way we were able to calculate the relative contribution to the effect of the MD for each dietary component. The greatest contribution derived from moderate alcohol drinking $(14.7 \%$ reduction of the effect, after elimination of this component from the score), high intake of cereals, monounsaturated fatty acids, vegetables and fruits and nuts $(12.2 \%, 5.8 \%$, $5.8 \%$ and $5.2 \%$ respectively) and reduced consumption of dairy and meat products $(13.4 \%$, and $3.4 \%$, respectively).

Besides MD, the main variables independently associated with higher risk of death, in a multivariable model, were sex (men), age, smoking habit and poor LTPA (see Table 1 in Supplementary Material).

\section{Discussion}

Our results from a large community sample indicate that adherence to a traditional MD was strongly 
associated with reduced overall and cardiovascular/ cerebrovascular mortality among subjects with prevalent diabetes.

Our findings confirm and expand the scarce literature on the topic. ${ }^{16,17,26}$ Studies conducted within an Australian cohort that included one-quarter of migrants born in Mediterranean countries, suggested that consumption of foods from the Mediterranean pattern may reduce cardiovascular mortality (CVD) and may be most beneficial for diabetics. ${ }^{16,17}$ However, one of these studies ${ }^{16}$ did not specifically assess the effect of a MD. Another study conducted in an Asian population suggested that a fish- and vegetable-rich diet had a favourable effect for diabetics but also in this case a traditional MD was not tested; ${ }^{18}$ more importantly, in that study the accounting of alcohol was not considered, despite being a fundamental component of the traditional Mediterranean pattern. ${ }^{1,25}$

We used both an 'a priori' and 'a posteriori' approach to characterize dietary habits. In the first case, a higher adherence to a traditional MD was significantly associated with reduced mortality whereas the a posteriori approach revealed a similar although not statistically significant trend. Besides the fact that they represent two different methodological approaches likely providing different results, the two scores included alcohol intake in a different way: the $a$ poster $i$ ori approach considered it as a continuous variable without any regard of the nonlinear association of alcohol with health outcomes; conversely, the a priori approach considered moderate alcohol intake as the optimal condition as highlighted by scientific evidence. ${ }^{27}$ Since in our study moderate alcohol intake resulted to be the major contributor to the effect of the MD, this dissimilar methodological approach may have generated the slightly different findings that we observed.

There is some good evidence in the literature on the benefits deriving from low-carbohydrate diets, ascribed to their role in promoting glycaemic ${ }^{28}$ and body weight control $^{29}$ or from a larger consumption of quality fats $^{30,31}$ or dietary fibres. ${ }^{32,33} \mathrm{We}$ investigated the relative importance of individual components of the MD in generating the inverse association of increased adherence to this diet and overall mortality. As dietary intake is a multiple exposure variable and people eat foods with complex combinations of nutrients, this approach is likely to have a greater potential for studying the association of diet with health outcomes.

The greatest contribution to the preventive effect of a MD on overall mortality mainly derived from moderate alcohol consumption, high intake of cereals, vegetables, fruits and nuts, a larger use of monounsaturated fatty acids rather than saturated fats and reduced consumption of dairy and meat products. The positive contribution of moderate alcohol has already been highlighted by findings indicating a beneficial role in not only lowering the risk of diabetes but also the incidence of heart disease in persons with diabetes. ${ }^{34,35}$ Consistently, health advantages from cereal fibre intake in lowering incident diabetes have been widely emphasized. ${ }^{32}$

Similarly, our findings on the positive impact of reduced meat and meat products on mortality are in accordance with previous evidence linking high intake of these foods to increased risk of the illness. ${ }^{36}$ Conversely, studies on the consumption of dairy products are inconclusive and sparse, although some beneficial effect was detected for fermented dairy product intake. $^{37}$

As suggested by a recent meta-analysis, interventions targeting pre-diabetes did not provide any significant reduction in all-cause or cardiovascular mortality. ${ }^{38}$ However, none of the dietary interventions tested the effects of a Mediterranean-like eating pattern; on the contrary, they were basically aimed at lowering fat, alcohol and sugar intake and to increase fibre and vegetable consumption.

Intervention studies have already shown that MDinspired diets might improve the cardiovascular profile among diabetic patients ${ }^{39}$ but data on cardiovascular mortality (major cause of death for people suffering from diabetes) are lacking.

As far as we know, our study is the first to formally show an inverse and significant association between adherence to a traditional MD and cardiovascular/cerebrovascular mortality among diabetic patients.

\section{Strengths and limitations of this study}

The strengths of this study, which derives from a large population-based cohort, are represented by its prospective nature and by the comprehensive dietary approach based on two different measurements, both considering the quality of diet as a complex interaction of foods and nutrients and not as mere sum of them. By using either the a priori or the a posteriori methods we offered a more reliable picture of the diet quality of our population.

In addition, the further control of all analyses by blood glucose level and time of diagnosis of diabetes should ensure that the observed effect is independent of duration and severity of diabetes.

A major limitation of the present study is the relatively small number of recorded deaths, especially when cardiovascular deaths were considered, but this did not prevent statistically significant differences to be consistently observed. On the other hand, our sample size and number of events are comparable to those reported by other studies in this field. ${ }^{4,17}$ 
Finally, we acknowledge that dietary scores may not represent an extremely precise evaluation of the dietary habits of a given population; moreover, not all mortality events could be adequately validated.

\section{Conclusions}

The present investigation provides an original insight on the contribution of MD and its components in reducing mortality in diabetic patients. During the last decades the shift toward Western-type diets has characterized the Southern European countries too, traditionally linked to the Mediterranean eating model, and its adverse effects are mainly witnessed by increased rates of obesity and metabolic disorders, such as diabetes. ${ }^{40}$ Accruing evidence on the benefits of this traditional eating pattern may be of help, not only in lowering the incidence of diabetes but also in improving survival in those affected by this widespread disease with consequent gains in terms of cost/effectiveness of public health strategies.

\section{Acknowledgements}

The MOLI-SANI research group thanks Drs Vittorio Krogh and Sabina Sieri (Istituto Nazionale dei Tumori, Milan, Italy) for their contribution to dietary questionnaire analysis and interpretation, the Associazione Cuore Sano Onlus (Campobasso) for its financial support and the Azienda Sanitaria Regionale del Molise (ASReM, Campobasso, Italy), the Offices of vital statistics of the Molise region and the Molise Dati Spa (Campobasso, Italy) for their collaboration and support provided during the follow-up activities. Part of the work was presented at the Europrevent 2014 meeting held in Amsterdam on 8-10 May 2014 in the Epidemiology poster session (best poster award of the prevention and epidemiology session).

\section{Funding}

The enrolment phase of the MOLI-SANI study was performed at the Research Laboratories, Catholic University, Campobasso, Italy and was supported by unrestricted research grants from Pfizer Foundation (Rome, Italy) and the Italian Ministry of University and Research (MIUR, Rome, Italy) - Programma Triennale di Ricerca, Decreto no.1588. The funders had no role in study design, collection, analysis and interpretation of data, in the writing of the report and in the decision to submit the article for publication. All authors were and are independent from funders.

\section{Conflict of interest}

None declared.

\section{References}

1. Willett WC, Sacks F, Trichopoulou A, et al. Mediterranean diet pyramid: a cultural model for healthy eating. Am J Clin Nutr 1995; 61(6 Suppl.): 1402S-1406S.
2. Bonaccio M, Iacoviello L and de Gaetano G. The Mediterranean diet: the reasons for a success. Thromb Res 2012; 129: 401-404.

3. Sofi F, Abbate R, Gensini GF, et al. Accruing evidence on benefits of adherence to the Mediterranean diet on health: an updated systematic review and meta-analysis. Am J Clin Nutr 2010; 92: 1189-1196.

4. Trichopoulou A, Bamia C and Trichopoulos D. Mediterranean diet and survival among patients with coronary heart disease in Greece. Arch Intern Med 2005; 165: 929-935.

5. de Lorgeril M, Salen P, Martin JL, et al. Mediterranean dietary pattern in a randomized trial: prolonged survival and possible reduced cancer rate. Arch Intern Med 1998; 158: 1181-1187.

6. Esposito K, Maiorino MI, Ceriello A, et al. Prevention and control of type 2 diabetes by Mediterranean diet: a systematic review. Diabetes Res Clin Pract 2010; 89: 97-102.

7. Rossi M, Turati F, Lagiou P, et al. Mediterranean diet and glycaemic load in relation to incidence of type 2 diabetes: results from the Greek cohort of the population-based European Prospective Investigation into Cancer and Nutrition (EPIC). Diabetologia 2013; 56: 2405-2413.

8. Danaei G, Finucane MM, Lu Y, et al. National, regional, and global trends in fasting plasma glucose and diabetes prevalence since 1980: systematic analysis of health examination surveys and epidemiological studies with 370 country-years and 2.7 million participants. Lancet 2011; 378: $31-40$.

9. World Health Organization. Global status report on noncommunicable diseases 2010. Geneva: World Health Organization, 2011.

10. Franz MJ, Bantle JP, Beebe CA, et al. Nutrition principles and recommendations in diabetes. Diabetes Care 2004; 27(Suppl. 1): S36-S46.

11. Brunner EJ, Mosdøl A, Witte DR, et al. Dietary patterns and 15-y risks of major coronary events, diabetes, and mortality. Am J Clin Nutr 2008; 87: 1414-1421.

12. Salas-Salvadó J, Bulló M, Babio N, et al. Reduction in the incidence of type 2 diabetes with the Mediterranean diet: results of the PREDIMED-Reus nutrition intervention randomized trial. Diabetes Care 2011; 34: 14-19.

13. Esposito $\mathrm{H}$ and Giugliano D. Mediterranean diet and type 2 diabetes. Diabetes Metab Res Rev 2014; 30(Suppl. 1): 34-40.

14. Barzi F, Woodward M, Marfisi RM, et al. Mediterranean diet and all-causes mortality after myocardial infarction: results from the GISSI Prevenzione trial. Eur J Clin Nutr 2003; 57: 604-611.

15. Estruch R, Ros R, Salas-Salvadó J, et al. Primary prevention of cardiovascular disease with a Mediterranean diet. N Engl J Med 2013; 368: 1279-1290.

16. Harriss LR, English DR, Powles J, et al. Dietary patterns and cardiovascular mortality in the Melbourne Collaborative Cohort Study. Am J Clin Nutr 2007; 86: 221-229.

17. Hodge AM, English DR, Itsiopoulos C, et al. Does a Mediterranean diet reduce the mortality risk associated 
with diabetes: evidence from the Melbourne Collaborative Cohort Study. Nutr Metab Cardiovasc Dis 2011; 21: 733-739.

18. Iimuro S, Yoshimura $\mathrm{Y}$, Umegaki $\mathrm{H}$, et al. Japanese Elderly Diabetes Intervention Trial Study Group. Dietary pattern and mortality in Japanese elderly patients with type 2 diabetes mellitus: does a vegetable- and fishrich diet improve mortality? An explanatory study. Geriatr Gerontol Int 2012; 12 Suppl. 1: 59-67.

19. Cho SS, Qi L, Fahey GC Jr, et al. Consumption of cereal fiber, mixtures of whole grains and bran, and whole grains and risk reduction in type 2 diabetes, obesity, and cardiovascular disease. Am J Clin Nutr 2013; 98 : 594-619.

20. Iacoviello L, Bonanni A, Costanzo S, et al. The MOLISANI Project, a randomized, prospective cohort study in the Molise region in Italy; design, rationale and objectives. Italian J Public Health 2007; 4: 110-118.

21. Pala V, Sieri S, Palli D, et al. Diet in the Italian EPIC cohorts: presentation of data and methodological issues. Tumori 2003; 89: 594-607.

22. Pisani P, Faggiano F, Krogh V, et al. Relative validity and reproducibility of a food frequency dietary questionnaire for use in the Italian EPIC centres. Int $J$ Epidemiol 1997; 26(Suppl. 1): S152-S160.

23. Trichopoulou A, Costacou T, Bamia C, et al. Adherence to a Mediterranean diet and survival in a Greek population. $N$ Engl J Med 2003; 348: 2599-2608.

24. Centritto F, Iacoviello L, di Giuseppe R, et al. Dietary patterns, cardiovascular risk factors and $\mathrm{C}$-reactive protein in a healthy Italian population. Nutr Metab Cardiovasc Dis 2009; 19: 697-706.

25. Trichopoulou A, Bamia C and Trichopoulos D. Anatomy of health effects of Mediterranean diet: Greek EPIC prospective cohort study. BMJ 2009; 338: b2337.

26. Salas-Salvadó J, Bulló515 (25.8) M, Estruch R, et al. Prevention of diabetes with mediterranean diets: A subgroup analysis of a randomized trial. Ann Intern $\mathrm{Med}$ 2014; 160: 1-10.

27. Di Castelnuovo A, Costanzo S, Bagnardi V, et al. Alcohol dosing and total mortality in men and women: an updated meta-analysis of 34 prospective studies. Arch Intern Med 2006; 166: 2437-2445.

28. Dyason PA. A review of low and reduced carbohydrate diets and weight loss in type 2 diabetes. J Hum Nutr Diet 2008; 21: 530-538.
29. Nielsen JV and Joensson E. Low-carbohydrate diet in type 2 diabetes. Stable improvement of bodyweight and glycemic control during 22 months follow-up. Nutr Metab (Lond) 2006; 3: 22-26.

30. Hu FB, Manson JE, Stampfer MJ, et al. Diet, lifestyle, and the risk of type 2 diabetes mellitus in women. $N$ Engl J Med 2001; 345: 790-797.

31. Riserus U, Willett WC and Hu FB. Dietary fats and prevention of type 2 diabetes. Prog Lipid Res 2009; 48: 44-51.

32. Montonen J, Knekt P, Järvinen R, et al. Whole-grain and fiber intake and the incidence of type 2 diabetes. $A m J$ Clin Nutr 2003; 77: 622-629.

33. Venn BJ and Mann JI. Cereal grains, legumes and diabetes. Eur J Clin Nutr 2004; 58: 1443-1461.

34. Howard AA, Arnsten JH and Gourevitch MN. Effect of alcohol consumption on diabetes mellitus: a systematic review. Ann Intern Med 2004; 140: 211-219.

35. Koppes LL, Dekker JM, Hendriks HF, et al. Moderate alcohol consumption lowers the risk of type 2 diabetes: a meta-analysis of prospective observational studies. Diabetes Care 2005; 28: 719-725.

36. Micha R, Wallace SK and Mozaffarian D. Red and processed meat consumption and risk of incident coronary heart disease, stroke, and diabetes mellitus: a systematic review and meta-analysis. Circulation 2010; 121: 2271-2283.

37. Sluijs I, Forouhi NG, Beulens JW, et al. The amount and type of dairy product intake and incident type 2 diabetes: results from the EPIC-InterAct Study. Am J Clin Nutr 2012; 96: 382-390.

38. Hopper I, Billah B, Skiba M, et al. Prevention of diabetes and reduction in major cardiovascular events in studies of subjects with prediabetes: meta-analysis of randomised controlled clinical trials. Eur J Cardiovasc Prev Rehabil 2011; 18: 813-823.

39. Elhayany A, Lustman A, Abel R, et al. A low carbohydrate Mediterranean diet improves cardiovascular risk factors and diabetes control among overweight patients with type 2 diabetes mellitus: a 1-year prospective randomized intervention study. Diabetes Obes Metab 2010; 12: 204-209.

40. Berghöfer A, Pischon T, Reinhold T, et al. Obesity prevalence from a European perspective: a systematic review. BMC Public Health 2008; 8: 200. 



\section{CHAPTER 6.}

Low income is associated with poor adherence to a Mediterranean diet and a higher prevalence of obesity: cross-sectional results from the Moli-sani study

Bonaccio M, Bonanni AE, Di Castelnuovo A, De Lucia F, Donati MB, de Gaetano $\mathrm{G}$ and Iacoviello $\mathrm{L}$, on behalf of the Moli-sani Project Investigators

BMJ Open 2012;2:e001685. doi:10.1136/bmjopen-2012-001685 


\title{
BMJ Low income is associated with poor adherence to a Mediterranean diet and a higher prevalence of obesity: cross-sectional results from the Moli-sani study
}

\author{
Marialaura Bonaccio, ${ }^{1,2}$ Americo Ettore Bonanni, ${ }^{1}$ Augusto Di Castelnuovo, ${ }^{3}$ \\ Francesca De Lucia, ${ }^{1}$ Maria Benedetta Donati, ${ }^{2,4}$ Giovanni de Gaetano, ${ }^{2,4}$ \\ Licia lacoviello, ${ }^{2,3}$ on behalf of the Moli-sani Project Investigators
}

To cite: Bonaccio $\mathrm{M}$, Bonanni $\mathrm{AE}, \mathrm{Di}$ Castelnuovo A, et al. Low income is associated with poor adherence to a Mediterranean diet and a higher prevalence of obesity: cross-sectional results from the Moli-sani study. BMJ Open 2012;2:e001685. doi:10.1136/bmjopen-2012 001685

- Prepublication history and additional material for this paper are available online. To view these files please visit the journa online (http://dx.doi.org/10.1136/ bmjopen-2012-001685).

Received 20 June 2012 Accepted 27 September 2012

This final article is available for use under the terms of the Creative Commons Attribution Non-Commercial 2.0 Licence; see http://bmjopen.bmj.com

MOLI-SANI Project Investigators are listed in the online supplementary appendix S1 (Web-only file)

For numbered affiliations see end of article

Correspondence to Licia lacoviello; licia. iacoviello@moli-sani.org

\section{ABSTRACT}

Objectives: To examine cross-sectional associations of socioeconomic status (ie, income and education) with an adherence to a Mediterranean dietary pattern and obesity prevalence.

Design: Cross-sectional study on a sample of Italian subjects enrolled in the Moli-sani Project, a populationbased cohort study. The Italian EPIC food frequency questionnaire was used to determine food intake. Adherence to a Mediterranean diet (MD) was appraised according to both the Mediterranean score elaborated by Trichopoulou (MDS) and the novel Italian Mediterranean Index (IMI) and to the a posteriori scores derived from principal component analysis. Four income categories were identified.

Setting: Molise region, Italy.

Participants: 13262 subjects (mean age $53 \pm 11,50 \%$ men) out of 24318 citizens (age $\geq 35$ ) randomly enrolled in the Moli-sani Project.

Main outcomes: Dietary patterns and risk factors for cardiovascular disease.

Results: Household higher income were significantly associated with greater adherence to an MD $(p<0.0001)$ and to Olive oil and Vegetables dietary pattern in a multivariable model including age, sex daily energy intake, body mass index, physical activity, smoking, alcohol consumption, education and marital status. The odds of having the highest adherence to an MD clearly increased according to income levels. People having the highest income had 54\% $(95 \% \mathrm{Cl}$ $21 \%$ to $97 \%$, MDS) or $72 \%$ (95\% Cl $34 \%$ to $121 \%$, IMI) higher probability to stick to an MD-like eating pattern than those in the lowest-income group. Obesity prevalence was higher in the lowest-income group $(36 \%)$ in comparison with the highest-income category $(20 \%, p<0.0001)$. Income was associated with dietary patterns in all categories of education.

Conclusions: A higher income and education are independently associated with a greater adherence to MD-like eating patterns and a lower prevalence of obesity.

\section{ARTICLE SUMMARY}

Article focus

- Examining cross-sectional associations of socioeconomic status (ie, income and education) with an adherence to a Mediterranean dietary pattern and obesity prevalence in a sample of Italian subjects.

\section{Key messages}

- Higher income and education are independently associated with a greater adherence to Mediterranean diet-like eating patterns and a lower prevalence of obesity.

- Diet quality showed a continued improvement across a relatively small range of economic strata.

- These results may foster a discussion on healthy food accessibility in terms of economic costs.

Strengths and limitations of this study

- The strengths are represented by the very large population sample and by the fact that this topic was addressed by using two a priori Mediterranean scores and the a posteriori dietary patterns derived from the principal components analysis. This leads to overcoming the limitations each of these approaches may present. A major limitation is that people self-reported their own income and a high percentage of nonrespondent subjects.

\section{INTRODUCTION}

Mediterranean diet (MD) has been shown to offer protection against cardiovascular diseases (CVD), some types of cancer and neurodegenerative diseases in observational epidemiological studies. ${ }^{1}$ The Lyon Diet Heart Study, by a randomised dietary intervention, also showed the health benefits of an MD in secondary prevention. ${ }^{23}$ The main 
food components of the MD are vegetables, fruits, cereals and fish and olive oil as the main fat sources and a moderate amount of red wine. Recently, the UNESCO committee inscribed it on the list of Intangible Heritage. ${ }^{4}$

Despite the widely proven benefits of the diet discovered by Ancel Keys ${ }^{5}$ in the 50 s, the Southern European countries in which MD originated are rapidly withdrawing from this eating pattern orienting their food choices towards products typical of the Western diet which is rich in refined grains, saturated fats, sugars, red and processed meat. ${ }^{6}$ The reasons why people keep on drifting from one dietary regimen to another remain open to several hypotheses. ${ }^{7}$ Social changes appear to have contributed to radical reversal in dietary habits in Western and Southern European societies although developing countries are slightly turning to westernised diets as well. ${ }^{8}$

The cost of MD seem to have led people to give up this eating pattern in favour of less-expensive products which allow to save money, but are definitively unhealthy. ${ }^{9}$

Many studies suggest that diet quality follows a socioeconomic gradient highlighting how disadvantaged people present higher rates of obesity, diabetes, CVD and some types of cancer. ${ }^{10}$

The abandoning of MD is also considered as a possible cause of the increasing obesity pandemic. ${ }^{11}$ Several studies took a step forward to see whether there is an association between diet cost and obesity, and found that a higher adherence to healthy dietary patterns is linked to higher monetary costs and is inversely associated with body mass index (BMI) and obesity. ${ }^{12}$

The aim of the present study was to examine crosssectional associations of socioeconomic status (SES; ie, income and education) with adherence to a Mediterranean dietary pattern, with the perspective that encouraging people to adopt healthy eating behaviours would not just be a matter of good will but mainly an issue to develop concrete measures of intervention in terms of economic availability. Moreover, our study investigated a potential relationship among a low-income, an MD and obesity.

\section{MATERIAL AND METHODS}

Study population

The Moli-sani Project is a population-based cohort study which randomly recruited 24325 citizens of Molise, a region placed between Central and Southern Italy. The study enrolled men and women aged $\geq 35$ years, between March 2005 and April 2010, randomly recruited from subjects included in the city-hall registries of Molise. ${ }^{13}$ Exclusion criteria were pregnancy, disturbances in understanding/willing processes, ongoing poly-traumas or coma, refusal to sign the informed consent. The cohort will be followed up for incident cardiovascular and tumour events.

After exclusion of subjects reporting a personal history of CVD (angina, myocardial infarction, heart failure, revascularisation procedures and stroke) $(5.7 \%)$, cancer $(3.1 \%)$ or diabetes $(6 \%)$ and of those for whom no information on income $(30.7 \%)$ was available, mainly because they refused to answer or did not possess any reliable information on this issue, 13262 subjects were analysed. The latter were comparable with the entire Moli-sani Project population in terms of Mediterranean dietary patterns and socioeconomic features, whereas the mean age of the sample was slightly lower $(53.3 \pm 11$ vs $55 \pm 12)$ and had a higher number of men $(50 \%$ vs $48 \%$ ) in the entire sample.

\section{Dietary information}

The validated Italian EPIC food frequency questionnaire was used for evaluating the food intake. ${ }^{14} 15$ The questionnaire, computerised with tailor-made software, allowed to interview participants in an interactive way, including illustrations of sample dishes of definite sizes or by a reference to standard portions. To simplify the interpretation of data and to minimise within-person variations in intake of individual food items, 188 food items were classified into 45 predefined food groups on the basis of similar nutrient characteristics or culinary usage (see online supplementary appendix S2; Web-only file).

Moderate alcohol intake was defined as 'regularly drinking less than two or one drinks a day', by men and women, respectively.

Food consumption patterns were generated by using principal components analysis (PCA) conducted on the correlation matrix of 45 food groups. ${ }^{16}$ Three main factors emerged, in agreement with previous findings in the same population. ${ }^{16}$ The first pattern, identified as 'Olive Oil and Vegetables', was characterised by highpositive loadings for olive oil, vegetables, legumes, soups, fruits and fish. The second pattern, named as 'Pasta and Meat', was characterised by high-positive loadings for pasta, cooked tomatoes, red meat, animal fats and alcoholic beverages, and negative loadings for breakfast cereals and yogurt. The 'Eggs and Sweets' pattern was characterised by high-positive loadings for eggs, margarines, processed meat and sugar and sweets.

We evaluated the adherence to an MD by using the Mediterranean Diet Score (MDS) elaborated by Trichopoulou et al. ${ }^{17}$ Scoring was based on the intake of the following nine items: vegetables, legumes, fruits and nuts, dairy products, cereals, meat and meat products, fish, alcohol and the ratio of monounsaturated:saturated fats. For most items, consumption above the study median received 1 point; all other intakes received 0 points. For dairy products, meat and meat products, consumption below the median received 1 point. Medians are gender specific. For ethanol, men who consumed $10-50 \mathrm{~g} /$ day and women who consumed $5-25 \mathrm{~g} /$ day received 1 point; otherwise, the score was 0 . The possible scores ranged between 0 and 9 , the latter reflecting the maximal adherence.

We also used a new Italian Mediterranean Index (IMI) the scoring of which is based on the intake of 11 items: 
a high intake of six typical Mediterranean foods (pasta; typical Mediterranean vegetables such as raw tomatoes, leafy vegetables, onions, and garlic, salads, and fruiting vegetables; fruit; legumes; olive oil and fish); a low intake of four non-Mediterranean foods (soft drinks, butter, red meat and potatoes); and alcohol consumption. If the consumption of typical Mediterranean foods was in the third tertile of the distribution, the person received 1 point; all other intakes received 0 points. If the consumption of non-Mediterranean foods was in the first tertile of the distribution, the person received 1 point. Ethanol received 1 point for intake up to $12 \mathrm{~g} /$ day; abstainers and persons who consumed $>12 \mathrm{~g} /$ day received 0 points. Possible scores ranged from 0 to $11 .^{18}$ Such an Italian index was conceived to a better capture of healthy eating including foods, such as pasta, more typically available in Italy.

\section{Data collection}

BMI was calculated as $\mathrm{kg} / \mathrm{m}^{2}$. Waist circumferences were measured according to the National Institutes of Health, Heart, Lung and Blood guidelines. ${ }^{19}$ Blood pressure (BP) was measured by an automatic device (OMRON-HEM-705CP) three times on the nondominant arm, with the patient lying down for about $5 \mathrm{~min}$. Hypertension was defined as systolic $\mathrm{BP} \geq 160 \mathrm{~mm} \mathrm{Hg}$ and/or diastolic $\mathrm{BP} \geq 95 \mathrm{~mm} \mathrm{Hg}$, or by using a pharmacological treatment. Physical activity was assessed by a structured questionnaire (24 questions on working and leisure time and sports participation), and expressed as daily energy expenditure in metabolic equivalent task-hours (MET/day).

Serum lipids and glucose were assayed by enzymatic reaction methods using an automatic analyser (ILab 350, Instrumentation Laboratory (IL), Milan, Italy). Low-density lipoprotein-cholesterol was calculated according to Friedewald. High-sensitivity $\mathrm{C}$ reactive protein (CRP) was measured in a fresh serum, by a latex particle-enhanced immunoturbidimetric assay (IL Coagulation Systems on ACL9000). Interday and intraday coefficients of variables were $5.5 \%$ and $4.17 \%$, respectively.

\section{Socioeconomic variables}

Household net income categories were considered as low $(<10000$ Euro/year $)$, low-medium $\quad(>10000$ $<25000$ Euro/year), medium-high $(>25000<40000$ Euro/year) and high ( $>40000$ Euro/year).

Education level was divided into three categories: $\leq 8$ (low), $>8$ and $\leq 13$ (medium) and $>13$ (high) years of studies.

SES was expressed as a score based on five variables: dwelling ownership and ratio between the number of rooms and number of living-in family members (rooms per person), both currently and during childhood - and availability of hot water at home during childhood. The five components were dichotomised according to the median value, and a score of 1 was attributed to the cat egory supposed to be the marker of a higher social status in comparison with the opposite category: thus, we assigned a score of 1 to people living in a house with room density/living-in family members $>0.6$ or dwelling ownership or with availability of hot water, and a score 0 to people with room density/living-in family members $\leq 0.6$, no dwelling ownership or with unavailability of hot water. The SES score did not include income and education. Marital status was considered as married or live-in partner versus others (divorced, unmarried and widower).

\section{Statistical analysis}

Values for continuous variables are means \pm SD. CRP was transformed into natural logarithm to reduce positive skewness, but data were reported untransformed for clarity. Analysis of variance for continuous or categorical variables was applied to test the associations in table 1 . A multivariable analysis of variance with appropriate terms for interaction was used for testing the association of adherence to MD scores, dietary patterns or dietary variables (considered as the dependent variables) with categories of income or SES components. By using multivariable logistic regression analysis (with appropriate terms for interaction), OR with a corresponding 95\% CI were calculated to quantify the association of income or education levels with adherence to MD-like eating scores or obesity. A high adherence to an MD, as stated by the Medscore, was defined when the score was $\geq 6$ points whereas a low adherence when the score was $\leq 3$ points. Subjects with intermediate values (4 or 5 points) were excluded from this analysis to focus on the two extreme categories of adherence. The same was done for the IMI score, but the cut-off was $\geq 5$ for the higher or $\leq 3$ for the lower adherence category.

The data analysis was generated using the SAS/STAT software, V.9.1.3 of the SAS System for Windows2009. SAS Institute Inc and SAS are registered trademarks of SAS Institute Inc, Cary, North Carolina, USA.

\section{RESULTS}

\section{Income groups}

Table 1 shows the characteristics of the entire population by income categories. People in the uppermost income group were $53.5 \%$ men and showed a better health profile, having significantly lower values of BMI, systolic BP, CRP, triglycerides and blood glucose. Obesity prevalence (BMI $>30 \mathrm{~kg} / \mathrm{m}^{2}$ ) varied according to the income; it was higher in the lowest-income group $(36 \%)$ and lower in the highest-income category $(20 \%, \mathrm{p}<0.0001$ table 1).

In table 2 the association among income levels, dietary habits and single food groups are reported.

Higher income groups were significantly associated with a greater adherence to both score indexes, namely MDS $(\mathrm{p}<0.0001)$ and IMI $(\mathrm{p}<0.0001)$ in the model adjusted for age, sex, daily energy intake, BMI, physical 
Low income is associated with poor adherence to Mediterranean diet

\begin{tabular}{|c|c|c|c|c|c|c|}
\hline Income categories & $\begin{array}{l}\text { All } \\
(n=13262)\end{array}$ & $\begin{array}{l}<10000 \\
(n=980,7.4 \%)\end{array}$ & $\begin{array}{l}>10000<25000 \\
(n=5751,43.4 \%)\end{array}$ & $\begin{array}{l}>25000<40000 \\
(n=4120,31.1 \%)\end{array}$ & $\begin{array}{l}>40000 \\
(n=2411, \\
18.2 \%)\end{array}$ & p Value ${ }^{\star}$ \\
\hline Age (years) & $53.3(10.6)$ & $60.1(12.7)$ & $54.0(11.2)$ & $51.4(9.5)$ & $52.3(8.8)$ & $<0.0001$ \\
\hline Sex (males, n, \%) & 6590 (49.7\%) & 348 (35.5\%) & 2834 (49.3\%) & 2117 (51.4\%) & 1291 (53.5\%) & $<0.0001$ \\
\hline BMI $\left(\mathrm{kg} / \mathrm{m}^{2}\right)$ & $27.7(4.6)$ & $28.7(5.3)$ & $28.2(4.7)$ & $27.3(4.3)$ & $27.0(4.0)$ & $<0.0001$ \\
\hline WH-ratio & $0.91(0.07)$ & $0.92(0.079)$ & $0.91(0.075)$ & $0.91(0.075)$ & $0.91(0.074)$ & 0.019 \\
\hline $\begin{array}{l}\text { Systolic blood } \\
\text { pressure }(\mathrm{mm} \mathrm{Hg})\end{array}$ & $139.0(20.1)$ & $143.4(21.0)$ & $140.1(20.4)$ & $137.3(19.6)$ & $137.4(19.2)$ & 0.0053 \\
\hline $\begin{array}{l}\text { Diastolic blood } \\
\text { pressure }(\mathrm{mm} \mathrm{Hg})\end{array}$ & $82.7(9.6)$ & $81.7(9.7)$ & $82.8(9.4)$ & $82.7(9.7)$ & $82.8(10.0)$ & 0.0038 \\
\hline $\begin{array}{l}\text { Total physical } \\
\text { activity (MET-h/day) }\end{array}$ & $43.4(8.7)$ & $44.8(10.4)$ & $45.0(9.7)$ & $42.5(7.9)$ & $40.4(5.2)$ & $<0.0001$ \\
\hline $\begin{array}{l}\text { Leisure time PA } \\
\text { (MET-h/day) }\end{array}$ & $2.18(1.88)$ & $1.81(1.67)$ & $2.10(1.78)$ & $2.22(1.96)$ & $2.28(1.94)$ & 0.20 \\
\hline $\begin{array}{l}\text { Working PA (MET-h/ } \\
\text { day) }\end{array}$ & $14.6(12.2)$ & $22.4(19.1)$ & $18.8(14.3)$ & $12.7(10.4)$ & $10.2(6.4)$ & $<0.0001$ \\
\hline \multicolumn{7}{|l|}{ Smokers n, \% } \\
\hline Never & $6370(48.1 \%)$ & $558(56.9 \%)$ & 2838 (49.4\%) & 1901 (46.2\%) & $1073(44.5 \%)$ & $<0.0001$ \\
\hline Current & $3296(25.0 \%)$ & $222(22.6 \%)$ & 1418 (24.7\%) & $1060(25.8 \%)$ & $596(24.7 \%)$ & \\
\hline Former & 3585 (27.5\%) & $200(20.4 \%)$ & $1490(25.9 \%)$ & $1154(28.0 \%)$ & 741 (30.7\%) & \\
\hline $\begin{array}{l}\text { Total cholesterol } \\
(\mathrm{mg} / \mathrm{dl})\end{array}$ & $212.3(40.2)$ & $214.3(41.9)$ & $212.8(40.4)$ & $211.8(40.4)$ & $211.0(38.7)$ & 0.64 \\
\hline HDL (mg/dl) & $57.0(14.5)$ & $58.4(14.4)$ & $57.2(14.4)$ & $56.6(14.5)$ & $56.8(14.6)$ & 0.35 \\
\hline LDL (mg/dl) & $130.3(33.6)$ & $130.8(35.7)$ & $130.3(33.8)$ & $130.2(33.6)$ & $130.1(32.7)$ & 0.65 \\
\hline CRP (mg/dl) & $2.4(3.0)$ & $3.0(3.7)$ & $2.6(3.1)$ & $2.2(2.9)$ & $2.1(2.7)$ & $<0.0001$ \\
\hline Triglycerides (mg/dl) & $127.0(83.8)$ & $129.1(87.0)$ & $129.1(84.5)$ & $126.1(85.0)$ & $122.6(78.4)$ & 0.0002 \\
\hline $\begin{array}{l}\text { Blood glucose (mg/ } \\
\text { dl) }\end{array}$ & $96.8(17.0)$ & $97.7(20.4)$ & $97.2(16.8)$ & $96.4(16.5)$ & $96.4(16.4)$ & 0.17 \\
\hline Obesity (n, \%) & 3563 (26.9\%) & $352(36.0 \%)$ & $1733(30.1 \%)$ & 988 (24.0\%) & $490(20.3 \%)$ & $<0.0001$ \\
\hline Hypertension (n, \%) & $4469(33.7 \%)$ & $452(46.2 \%)$ & 2006 (34.9\%) & 1259 (30.6\%) & $752(31.2 \%)$ & 0.79 \\
\hline
\end{tabular}

activity, smoking, alcohol consumption and marital status.

Similar data were obtained after stratification by gender ( $p$ for interaction $=0.24$ for MDS and $p$ for interaction $=0.41$ for IMI) and age ( $p$ for interaction $=0.43$ for IMI). However, the increasing adherence to MD according to income was more pronounced in the elderly when it was measured as MSD ( $p$ for interaction $=0.0002$; $\beta=0.063, \mathrm{SE} \pm 0.021, \mathrm{p}=0.0028$ for people $\leq 65$ years and $\beta=0.17, \mathrm{SE} \pm 0.051, p=0.0008$, for people $>65$ years $)$.

Subjects in the lower-income categories showed poor adherence to the Olive oil and Vegetables dietary pattern $(\mathrm{p}<0.0001)$, while a greater adherence to the Western-type pattern (Dietary pattern 3) was observed. Similar results were observed after stratification for gender and age (data not shown).

In addition, an analysis of single foods consumption by income categories showed that people with a higher income reported higher intake of the basic components of an MD, that is fish, fruits, legumes and a lower consumption of animal fats, processed meat and white meat the frequency of consumption of which is more typical of the Western dietary model.
The odds of having the highest adherence to the MD that increased with both MDS and IMI scores, clearly increased according to income levels (table 3). People having the highest income had 54\% (MDS) or 72\% (IMI) a statistically significant higher probability to stick to an MD-like eating pattern than those in the lowest-income group (table 3).

Regarding alcohol consumption (table 2), the highest income group appeared to include the highest prevalence of moderate drinkers $(41.7 \%$ vs $27.5 \%$, recorded in the lowest-income group) as already found in a sample of Danish population. ${ }^{20}$

\section{SES and education}

Income fairly correlated with SES (Spearman correlation coefficients $=0.24, \quad \mathrm{p}<0.0001)$ and education $(\mathrm{r}=0.51$, $\mathrm{p}<0.0001)$, whereas correlation between SES and education was $\mathrm{r}=0.34, \mathrm{p}<0.0001$.

Education was positively associated with adherence to MD, measured by both MDS $(p=0.034)$ and IMI $(\mathrm{p}=0.0014)$, while SES was not $(\mathrm{p}=0.19$ for MDS and $\mathrm{p}=0.78$ for IMI) in the fully adjusted model also including education and income. 
Low income is associated with poor adherence to Mediterranean diet

\begin{tabular}{|c|c|c|c|c|c|c|}
\hline Income categories & All $(n=13262)$ & $\begin{array}{l}<10000 \\
(n=980,7.4 \%)\end{array}$ & $\begin{array}{l}>10000<25000 \\
(n=5751,43.4 \%)\end{array}$ & $\begin{array}{l}>25000<40000 \\
(n=4120,31.1 \%)\end{array}$ & $\begin{array}{l}>40000 \\
(n=2411, \\
18.2 \%)\end{array}$ & p Value * \\
\hline $\begin{array}{l}\text { Mediterranean score } \\
\text { (MDS) }\end{array}$ & $4.44(1.64)$ & $4.32(1.61)$ & $4.40(1.62)$ & $4.46(1.62)$ & $4.53(1.70)$ & $<0.0001$ \\
\hline $\begin{array}{l}\text { Italian Mediterranean } \\
\text { index (IMI) }\end{array}$ & $3.26(1.71)$ & $3.20(1.64)$ & $3.15(1.68)$ & $3.30(1.71)$ & 3.49 (1.79) & $<0.0001$ \\
\hline $\begin{array}{l}\text { Dietary pattern } 1 \\
\text { (olive oil and } \\
\text { vegetables) }\end{array}$ & $0.042(0.95)$ & $-0.066(0.92)$ & $0.021(0.94)$ & $0.070(0.95)$ & $0.091(0.97)$ & $<0.0001$ \\
\hline $\begin{array}{l}\text { Dietary pattern } 2 \\
\text { (pasta and meat) }\end{array}$ & $0.036(0.95)$ & $0.0078(0.91)$ & $0.093(0.95)$ & $0.0053(0.95)$ & $-0.036(0.97)$ & $<0.0001$ \\
\hline $\begin{array}{l}\text { Dietary pattern } 3 \\
\text { (eggs and sweets) }\end{array}$ & $0.015(0.85)$ & $-0.13(0.88)$ & $0.040(0.86)$ & $0.044(0.85)$ & $-0.033(0.82)$ & $<0.0001$ \\
\hline $\begin{array}{l}\text { Energy intake (kcal/ } \\
\text { day) }\end{array}$ & $2177.4(640.7)$ & $2062.5(704.1)$ & $2186.3(649.0)$ & $2190.6(627.8)$ & $2180.4(610.7)$ & 0.58 \\
\hline $\begin{array}{l}\text { Alcohol intake (g/ } \\
\text { day) }\end{array}$ & $16.5(22.4)$ & $16.1(25.8)$ & $18.2(24.5)$ & $15.3(20.3)$ & $14.9(18.6)$ & $<0.0001$ \\
\hline $\begin{array}{l}\text { Moderate drinkers } \\
(\mathrm{n}, \%)\end{array}$ & $4303(33.3 \%)$ & $261(27.5 \%)$ & $1646(29.2 \%)$ & $1419(35.3 \%)$ & $977(41.7 \%)$ & $<0.0001$ \\
\hline $\begin{array}{l}\text { Wine consumption } \\
\text { (ml/day) }\end{array}$ & $135.5(188.7)$ & $133.3(209.6)$ & $148.9(203.7)$ & $125.5(175.4)$ & $121.8(160.4)$ & $<0.0001$ \\
\hline Olive oil (g/day) & $24.2(9.2)$ & $22.9(9.0)$ & $24.4(9.3)$ & $24.4(9.2)$ & $24.0(9.1)$ & 0.0034 \\
\hline Animal fat (g/day) & $1.26(1.41)$ & $1.21(1.37)$ & $1.34(1.45)$ & $1.25(1.41)$ & $1.11(1.31)$ & $<0.0001$ \\
\hline Fish (g/day) & $20.9(17.0)$ & $17.5(15.5)$ & $19.4(16.3)$ & $22.1(17.4)$ & $23.9(18.0)$ & $<0.0001$ \\
\hline $\begin{array}{l}\text { Processed meat ( } \mathrm{g} / \\
\text { day) }\end{array}$ & $30.4(20.9)$ & $27.4(20.0)$ & $31.5(21.8)$ & $30.7(20.5)$ & $28.6(19.9)$ & $<0.0001$ \\
\hline $\begin{array}{l}\text { Cooked vegetables } \\
\text { (g/day) }\end{array}$ & $73.5(43.4)$ & $71.2(42.8)$ & $74.8(44.0)$ & $74.0(42.5)$ & $70.6(43.4)$ & 0.0006 \\
\hline Legumes (g/day) & $28.3(22.1)$ & $27.1(20.3)$ & $27.1(21.6)$ & $28.4(21.9)$ & $31.3(24.2)$ & $<0.0001$ \\
\hline $\begin{array}{l}\text { Nuts and seeds (g/ } \\
\text { day) }\end{array}$ & $0.89(2.3)$ & $0.90(3.2)$ & $0.87(2.3)$ & $0.88(2.1)$ & $0.94(2.2)$ & 0.34 \\
\hline Red meat (g/day) & $47.8(26.0)$ & $44.7(26.3)$ & $48.8(25.9)$ & $47.9(25.8)$ & $46.8(26.3)$ & 0.0008 \\
\hline White meat (g/day) & $26.4(18.8)$ & $29.6(19.8)$ & $28.1(19.3)$ & $25.3(17.8)$ & $23.2(18.1)$ & $<0.0001$ \\
\hline Fruits (g/day) & 358.5 (204.3) & $362.7(211.1)$ & 354.9 (201.2) & 355.7 (198.9) & $370.3(217.0)$ & 0.015 \\
\hline $\begin{array}{l}\text { Crustaceans, } \\
\text { molluscs, seafood } \\
\text { (g/day) }\end{array}$ & $11.0(10.1)$ & $8.9(8.8)$ & $10.7(9.9)$ & $11.7(10.7)$ & $11.5(10.0)$ & $<0.0001$ \\
\hline $\begin{array}{l}\text { Vegetable oils (no } \\
\text { olive; g/day) }\end{array}$ & $0.28(0.81)$ & $0.33(1.23)$ & $0.29(0.79)$ & $0.28(0.69)$ & $0.27(0.84)$ & 0.18 \\
\hline $\begin{array}{l}\text { Refined grains } \\
\text { (pasta and bread; (g/ } \\
\text { day) }\end{array}$ & $198.3(101.9)$ & $193.5(104.5)$ & $201.3(103.3)$ & $196.3(99.2)$ & $196.8(101.9)$ & 0.0004 \\
\hline Sugar (g/day) & $11.0(10.4)$ & $10.3(9.7)$ & $11.2(10.9)$ & $11.1(10.3)$ & $10.4(9.8)$ & $<0.0001$ \\
\hline
\end{tabular}

Odds of having a higher adherence according to education levels were 1.26 and 1.27 ( $p$ for trend $=0.0020$ ) for MDS and 1.16 and 1.33 ( $\mathrm{p}$ for trend=0.0009) for IMI (table 3).

\section{Stratification by education}

As less-educated people may show a lower adherence to an MD owing to the lack of knowledge about healthy habits, ${ }^{21}$ we performed additional analyses stratified for educational level.

Either in lower $(<=8)$, medium $(>8$ and $\leq 13)$ and highly (>13 years of studies) educated groups, adherence to an MD followed the gradient of income categories (table 4), with the exception of the uppermost educated group, when the MDS score was used ( $p=0.067)$. However, the interaction test was not significant for either score. Accordingly, education was related to the dietary pattern independently from income. Indeed, by dividing income levels into two main categories (low and low-medium vs high and high-medium), in the fully adjusted model, education was positively associated with dietary patterns both in the lowest (MDS: $\mathrm{p}=0.032$ and IMI: $\mathrm{p}=0.0025)$ and in the highest income groups (MDS: $\mathrm{p}=0.0067$ and IMI: $\mathrm{p}=0.0010$ ). 
Low income is associated with poor adherence to Mediterranean diet

\begin{tabular}{|c|c|c|c|c|c|c|c|c|}
\hline \multirow[b]{2}{*}{ Income } & \multicolumn{4}{|c|}{ Mediterranean score } & \multicolumn{4}{|c|}{ Italian Mediterranean Index } \\
\hline & $\begin{array}{l}\text { Low } \\
(n=3843)\end{array}$ & $\begin{array}{l}\text { High } \\
(n=3518)\end{array}$ & OR & $(95 \% \mathrm{Cl})$ & $\begin{array}{l}\text { Low } \\
(n=4704)\end{array}$ & $\begin{array}{l}\text { High } \\
(n=3089)\end{array}$ & OR & (95\% Cl) \\
\hline$<10000$ & $310(8.1 \%)$ & $231(6.6 \%)$ & $-1-$ & (referent) & $353(7.5 \%)$ & $209(6.8 \%)$ & $-1-$ & (referent) \\
\hline$>10000<25000$ & $1694(44.1 \%)$ & $1470(41.8 \%)$ & 1.28 & (1.04 to 1.57$)$ & $2161(45.9 \%)$ & $1201(38.9 \%)$ & 1.07 & (0.87 to 1.32$)$ \\
\hline$>25000<40000$ & $1153(30.0 \%)$ & $1116(31.7 \%)$ & 1.51 & (1.21 to 1.88 ) & $1436(30.5 \%)$ & 995 (32.2\%) & 1.34 & (1.07 to 1.69 ) \\
\hline$>40000$ & $686(17.9 \%)$ & $701(19.9 \%)$ & $\begin{array}{l}1.54 \\
\text { ( } p \text { for } \\
\text { trend }=0.0002 \text { ) }\end{array}$ & (1.21 to 1.97$)$ & $754(16.0 \%)$ & $684(22.1 \%)$ & $\begin{array}{l}1.72 \\
\text { ( } p \text { for } \\
\text { trend }<0.0001 \text { ) }\end{array}$ & (1.34 to 2.21 ) \\
\hline \multicolumn{9}{|l|}{ Education level } \\
\hline Low & $1775(46.2 \%)$ & $1571(44.7 \%)$ & $-1-$ & (referent) & 2250 (47.8\%) & $1307(42.3 \%)$ & $-1-$ & (referent) \\
\hline Medium & $1504(39.1 \%)$ & $1421(40.4 \%)$ & 1.26 & (1.11 to 1.43 ) & $1860(39.6 \%)$ & $1283(41.5 \%)$ & 1.16 & (1.02 to 1.31 ) \\
\hline High & $564(14.7 \%)$ & 525 (14.9) & $\begin{array}{l}1.27 \\
(p \text { for } \\
\text { trend=0.0020) }\end{array}$ & (1.06 to 1.52$)$ & $592(12.6 \%)$ & $499(16.1 \%)$ & $\begin{array}{l}1.33 \\
\text { ( } p \text { for } \\
\text { trend=0.0009) }\end{array}$ & (1.11 to 1.60$)$ \\
\hline \multicolumn{9}{|l|}{$\begin{array}{l}\text { Socioeconomic } \\
\text { status }\end{array}$} \\
\hline
\end{tabular}

\section{Income/education, an MD and obesity}

Finally, we evaluated the impact of income and education on the diet quality and then on obesity in a unique statistical model. OR of being obese decreased according to income $(\mathrm{OR}=0.72,95 \% \mathrm{CI} 0.59$ to 0.86 for the highest-income vs the lowest-income group) and education level $(\mathrm{OR}=0.53,95 \% \mathrm{CI} 0.45$ to 0.61 for the highest vs the lowest education level). These ORs remained unchanged when in the model diet quality (measured as Mediterranean scores) was included. Moreover, the association between income or education and obesity was equally observed in both the highest and lowest MD adherence categories as defined in tables 3 and 4 (the four terms for interactions among income or education with MDS or IMI were all $\mathrm{p}>0.35$ ).

\section{DISCUSSION}

People with a higher income and higher levels of education had a greater adherence to MD-like eating patterns, as measured by three different parameters: two a priori
Mediterranean scores (the traditional one introduced by Trichopoulou and a more recent IMI), and the a posteriori dietary patterns derived from the PCA. Evidence on the health benefits of the MD is based on several studies and meta-analyses. ${ }^{1} 721$ However, adherence to this healthy eating pattern is rapidly disappearing in the countries of Southern Europe where it originated and persisted for centuries, including the areas of Northern Africa in which there is an increasing prevalence of metabolic disorders and consequent CVD mainly owing to the changing lifestyle and/or habits. ${ }^{22}$ SES has been included among the factors related to chronic disease onset, and disparities in dietary habits by social class have been advocated to explain at least in part the higher CVD risk factors profile observed among low SES groups. ${ }^{23}$

Our results agree with the conclusions reported in the review by Darmon and Drewnowski ${ }^{10}$ that higher-quality diets are mainly consumed by better educated and more affluent people, while lower socioeconomic groups tend to have lower-quality diets thus exposing themselves to a

\begin{tabular}{|c|c|c|c|c|c|}
\hline & \multicolumn{4}{|c|}{ Income categories } & \multirow[b]{2}{*}{ p Value* } \\
\hline & $<10000$ & $>10000<25000$ & $>25000<40000$ & $>40000$ & \\
\hline Lower Education ( $n=6101,46.0 \%)$ & $(n=829)$ & $(n=3745)$ & $(n=1260)$ & $(n=267)$ & \\
\hline Mediterranean Diet & $4.33(1.60)$ & $4.43(1.63)$ & $4.44(1.60)$ & $4.59(1.64)$ & 0.040 \\
\hline Italian Mediterranean Index & $3.19(1.61)$ & $3.13(1.67)$ & $3.22(1.69)$ & $3.54(1.73)$ & 0.0002 \\
\hline Medium Education ( $n=5236,39.5 \%)$ & $(n=137)$ & $(n=1746)$ & $(n=2184)$ & $(n=1169)$ & \\
\hline Mediterranean Diet & $4.16(1.64)$ & $4.33(1.60)$ & $4.50(1.63)$ & $4.54(1.67)$ & 0.0041 \\
\hline Italian Mediterranean Index & $3.21(1.67)$ & $3.14(1.69)$ & $3.33(1.73)$ & $3.43(1.81)$ & $<0.0001$ \\
\hline Higher Education ( $n=1922,14.5 \%)$ & $(n=13)$ & $(n=258)$ & $(n=676)$ & $(n=975)$ & \\
\hline Mediterranean Diet & $5.61(1.85)$ & $4.43(1.61)$ & $4.38(1.60)$ & $4.50(1.76)$ & 0.067 \\
\hline Italian Mediterranean Index & 3.77 (2.68) & $3.43(1.63)$ & $3.33(1.70)$ & $3.56(1.77)$ & 0.041 \\
\hline
\end{tabular}


higher risk of developing diet-related diseases. Similar conclusions were reached by other investigations too 24 suggesting that low socioeconomic groups end up having poorer diets. These findings are supported, at least in part, by the fact that following a Mediterranean dietary style could represent a matter of money. ${ }^{9}$ Indeed researchers in Spain showed that an MD is definitely more expensive to follow than Western dietary patterns: this may represent a strong economic obstacle when counselling people about the opportunity to follow a healthy diet because cost may become a prohibitive factor. ${ }^{9}$ Aggarwal et $a l^{25}$ demonstrated that the wellknown socioeconomic disparities in diet quality is mediated by the food cost confirming that lower SES groups tend to consume more energy-dense and nutrient-poor diets. However, the economic advantages of a Mediterranean way of eating in terms of costeffectiveness should be highlighted as shown in patients with a previous CVD that could represent an exceptional return on investment. ${ }^{26}$

Subjects with a lower income had a greater prevalence of obesity too. The association between obesity and socioeconomic factors has been previously observed ${ }^{27}$ suggesting that the latter plays an important role in the risk of obesity and overweight not only in adults but also in children. ${ }^{28}$ However, our data show that the strong association observed between a lower income or education with obesity was not mediated by the diet quality. Indeed, an additional analysis combining the impacts of education or income on diet quality and then on obesity in a unique statistical model, showed that the association remained unchanged when diet quality was included. According to these results, the changes in obesity rates observed in different income and education categories appear not necessarily mediated by diet quality. However, the epidemiological evidence supporting a causal link between MDs and body weight is contrasting. ${ }^{29}$

It is quite clear that accumulating proofs on the benefits of Mediterranean-like diets is an insufficient prevention strategy as conditions allowing people to stick to healthier dietary habits should also be clearly identified.

This study contributes to provide further evidence for the assumption that dietary habits are strongly influenced by socioeconomic factors, in particular by income which appears to play an important role in determining people's food choices. ${ }^{30}$ As far as education is concerned, previous studies found a relationship between higher levels of education and healthy diets. ${ }^{31}$ In our research, education was found to be independently associated to MD and did not modify the association between income levels and a healthy dietary pattern as shown in the stratified analysis by education levels.

The promotion of healthy lifestyles and diets to prevent weight gain and related diseases has taken the top position in the priority list of the public health experts all over the world since obesity has become a life-threatening epidemic. So far the traditional MD has proven to be an effective 'remedy' to the spreading of major chronic diseases, obesity and mortality. Our study highlights the strong linkage among low income, poor adherence to MD and obesity prevalence.

\section{Limitations of this study}

A major limitation of the present study is that people selfreported their own income which is a quite sensitive issue. Indeed, we recorded a high percentage $(30.7 \%)$ of nonrespondent subjects who refused to declare or did not know their family income. Yet, such a large nonrespondent group is very common in this type of investigations, especially among women and the elderly. ${ }^{32}$ However, there was no difference between the entire Moli-sani population and the subsample analysed here as far as dietary habits and socioeconomic variables were concerned.

Another inherent limit is represented by the crosssectional nature of our study.

In addition, caution is needed in extending the results presented here to larger contexts since data were collected in a region located between Central and Southern Italy, Mediterranean by tradition and culture. ${ }^{13}$ Yet, the main characteristics of our population sample are comparable to those of the Italian Cardiovascular Epidemiological Observatory, ${ }^{33}$ a large survey including random samples of the general population recruited all over Italy; therefore our sample can be considered a representative at least of the whole Italian population.

\section{Strengths of this study}

Our very large population sample is composed of subjects coming from quite a homogeneous environment with no marked differences in terms of socioeconomic disparities, different from metropolitan areas, where previous studies found huge gaps among social classes and related health statuses at relatively small distances from the city centre. ${ }^{34}$ Bearing this in mind, the differences we observed in the adherence to MD according to income indicate that also in an environment homogeneous both for genetics and lifestyles, income and education can still play a role in influencing dietary choices. Furthermore, the diet quality showed a continued improvement across a relatively small range of economic strata. Our 'poorest' are represented by people earning less than 10000 Euro/net per year, while the 'richest' group is composed of subjects with more than 40000 Euro/net/year. Such differences among income classes are quite restrained and recall what has already been said for the pretty homogeneous environment where our sample comes from. We are not dealing with real huge socioeconomic and income differences. Despite this homogeneity, we did observe notable changes in diet quality among different groups.

The differences observed across the income strata would likely become even more evident in an MD importing countries where getting typical Mediterranean products is more difficult and expensive.

In addition, apparently for the first time this topic was addressed by using two a priori Mediterranean scores (the 
traditional one introduced by Trichopoulou and a novel IMI), and the a posteriori dietary patterns derived from the PCA. This leads to overcoming the limitations each of these approaches may present. Indeed, the 'a priori' scores only reflect some aspects of diet and do not account for correlations between score components. Instead, the 'a posteriori' approaches have the weakness of low reproducibility, different populations having different non-predefined dietary patterns. Therefore, the use of an index based on the foods actually available to Italians and traditional Italian cooking styles should improve the ability of the index to classify the Italian cohort.

\section{CONCLUSIONS}

Our data suggest consistent associations of income and education with dietary patterns and may foster a discussion on healthy food accessibility in terms of economic costs. The cost of an MD seem to represent a real obstacle to a healthy diet, driving people to choose alternative ways of eating usually inspired by the need to save money in everyday life. Public health policies shall take into account the fact that correct dietary habits need to be promoted by allowing people to choose the best for their own health. It is definitely an interdisciplinary issue which shall call to action every single actor of modern societies otherwise condemned to increase their already heavy burden of chronic diseases. As already noted by others who dealt with this topic, ${ }^{10}$ the promotion of high-cost foods to low-income people without taking food costs into account is not likely to be successful.

\section{Author affiliations}

${ }^{1}$ Science Communication Unit, Laboratory of Genetic and Environmental Epidemiology, Research Laboratories, Fondazione di Ricerca e Cura "Giovanni Paolo II", Campobasso, Italy

2Epicomed Research, Campobasso, Italy

${ }^{3}$ Laboratory of Genetic and Environmental Epidemiology, Research

Laboratories, Fondazione di Ricerca e Cura "Giovanni Paolo II", Campobasso, Italy

${ }^{4}$ Research Laboratories, Fondazione di Ricerca e Cura 'Giovanni Paolo II', Campobasso, Italy

Contributors All authors listed in the authorship (1) substantially contributed to the conception and design (MB, $\mathrm{AB}, \mathrm{LI}$ and $\mathrm{GdG}$ ), acquisition of data (FDL and $\mathrm{MB}$ ) or analysis and interpretation of data (MB, ADC and MBD); (2) draughted the article and revised it critically for important intellectual content and (3) approved the final version to be published. All authors had full access to all the data in the study and took responsibility for the integrity of the data and the accuracy of the data analysis. None of the authors had a personal or financial conflict of interest.

Funding The enrolment phase of the Moli-sani Project was supported by research grants from the Pfizer Foundation (Rome, Italy) the Italian Ministry of University and Research (MIUR, Rome, Italy)-Programma Triennale di Ricerca, Decreto no.1588, and Instrumentation Laboratory, Milan, Italy. Associazione Cuore-Sano Onlus contributed to the publication cost. Neither the Pfizer Foundation nor the MIUR had any role in the study design, collection, analysis and interpretation of the data; in the writing of the report; and in the decision to submit the article for publication. All the authors are independent from the funders.

Competing interest None.
Ethics approval The Moli-sani study was approved by the Ethics Committee of the Catholic University of Rome. Participants signed the informed consent before taking part in the study.

Provenance and peer review Not commissioned; externally peer-reviewed.

Data sharing statement № additional data are available.

\section{REFERENCES}

1. Sofi F, Abbate R, Gensini GF, et al. Accruing evidence on benefits of adherence to the Mediterranean diet on health: an updated systematic review and meta-analysis. Am J Clin Nutr 2010;92:1189-96.

2. de Lorgeril M, Salen P, Martin JL, et al. Mediterranean diet, traditional risk factors, and the rate of cardiovascular complications after myocardial infarction: final report of the Lyon Diet Heart Study. Circulation 1999;99:779-85.

3. de Lorgeril M, Salen P, Martin JL, et al. Mediterranean dietary pattern in a randomized trial: prolonged survival and possible reducedcancer rate. Arch Intern Med 1998;158:1181-7.

4. http://www.unesco.org/culture/ich/en/RL/00394 (accessed Nov 2011).

5. Keys A. Seven countries. A multivariate analysis of death, coronary heart disease. Cambridge, MA: Harvard University Press, 1980.

6. Laccetti R, Pota A, Stranges S, et al. Evidence on the prevalence and geographic distribution of major cardiovascular risk factors in Italy. Public Health Nutr 2012;30:1-11.

7. Bonaccio M, lacoviello L, de Gaetano G. The Mediterranean diet: the reasons for a success. Thromb Res 2012;129:401-4.

8. Prentice AM. The emerging epidemic of obesity in developing countries. Int J Epidemiol 2006;35:93-9.

9. Lopez CN, Martinez-Gonzalez MA, Sanchez-Villegas A, et al. Costs of Mediterranean and western dietary patterns in a Spanish cohort and their relationship with prospective weight change. J Epidemiol Community Health 2009;63:920-7.

10. Darmon N, Drewnowski A. Does social class predict diet quality? Am J Clin Nutr 2008;87:1107-17.

11. Esposito K, Kastorini CM, Panagiotakos DB, et al. Mediterranean diet and weight loss: meta-analysis of randomized controlled trials. Metab Syndr Relat Disord 2011:9:1-12.

12. Schröder H, Marrugat J, Covas MI. High monetary costs of dietary patterns associated with lower body mass index: a population-based study. Int J Obes (Lond) 2006;30:1574-9.

13. lacoviello L, Bonanni A, Costanzo S, et al. The Moli-sani Project, a randomized, prospective cohort study in the Molise region in Italy; design, rationale and objectives. Italian J Public Health 2007:4:110-18.

14. Pala V, Sieri S, Palli D, et al. Diet in the Italian EPIC cohorts: presentation of data and methodological issues. Tumori 2003;89:594-607.

15. Pisani $\mathrm{P}$, Faggiano $\mathrm{F}$, Krogh $\mathrm{V}$, et al. Relative validity and reproducibility of a food frequency dietary questionnaire for use in the Italian EPIC centres. Int J Epidemiol 1997;26(Suppl 1):S152-60.

16. Centritto F, lacoviello L, di Giuseppe R, et al. Dietary patterns, cardiovascular risk factors and C-reactive protein in a healthy Italian population. Nutr Metab Cardiovasc Dis 2009;19:697-706.

17. Trichopoulou A, Orfanos $\mathrm{P}$, Norat $\mathrm{T}$, et al. Modified Mediterranean diet and survival: EPIC-elderly prospective cohort study. BMJ 2005;330:991.

18. Agnoli C, Krogh V, Grioni S, et al. A priori-defined dietary patterns are associated with reduced risk of stroke in a large Italian cohort. J Nutr 2011;141:1552-8.

19. Janssen I, Katzmarzyk PT, Ross R. Body mass index, waist circumference, and health risk: evidence in support of current National Institutes of Health guidelines. Arch Intern Med 2002;162:2074-9.

20. Nielsen NR, Schnohr P, Jensen G, et al. Is the relationship between type of alcohol and mortality influenced by socio-economic status? $J$ Intern Med 2004;255:280-8.

21. Sofi F, Cesari F, Abbate R, et al. Adherence to Mediterranean diet and health status: meta-analysis. BMJ 2008;337:a1344.

22. Mehio Sibai A, Nasreddine L, Mokdad AH, et al. Nutrition transition and cardiovascular disease risk factors in Middle East and North Africa countries: reviewing the evidence. Ann Nutr Metab 2010;57:193-203.

23. Panagiotakos DB, Pitsavos C, Chrysohoou C, et al. Dietary habits mediate the relationship between socio-economic status and CVD factors among healthy adults: the ATTICA study. Public Health Nutr 2008;11:1342-9. 
24. Shahar D, Shai I, Vardi $\mathrm{H}$, et al. Diet and eating habits in high and low socioeconomic groups. Nutrition 2005;21:559-66.

25. Aggarwal A, Monsivais P, Cook AJ, et al. Does diet cost mediate the relation between socioeconomic position and diet quality? Eur J Clin Nutr 2011;65:1059-66.

26. Dalziel K, Segal L, de Lorgeril M. A mediterranean diet is cost-effective in patients with previous myocardial infarction. J Nutr 2006;136:1879-85.

27. McLaren L. Socioeconomic status and obesity. Epidemiol Rev 2007;29:29-48.

28. Shrewsbury V, Wardle J. Socioeconomic status and adiposity in childhood: a systematic review of cross-sectional studies 1990-2005. Obesity 2008;16:275-84.

29. Buckland G, Bach A, Serra-Majem L. Obesity and the Mediterranean diet: a systematic review of observational and intervention studies. Obes Rev 2008;9:582-93.
30. Darmon N, Ferguson EL, Briend A. A cost constraint alone has adverse effects on food selection and nutrient density: an analysis of human diets by linear programming. I Nut

2002;132:3764-71.

31. Groth MV, Fagt S, Brøndsted L. Social determinants of dietary habits in Denmark. Eur J Clin Nutr 2001;55:959-66.

32. Kaplan GA, Keil JE. Socioeconomic factors and cardiovascular disease: a review of the literature. Circulation 1993;88: disease: a

33. Giampaoli S, Rielli R, Dematté L, et al. The Italian observatory of cardiovascular risk: the CUORE project experience (abstract). Circulation 2009;119:139.

34. Diez-Roux AV, Nieto FJ, Caulfield L, et al. Neighbourhood differences in diet: the Atherosclerosis Risk in Communities (ARIC) Study. J Epidemiol Community Health 1999;53:55-63. 


\section{CHAPTER 7.}

Mass media information and adherence to Mediterranean diet: results from the Moli-sani study

Bonaccio M, Di Castelnuovo A, Costanzo S, De Lucia F, Olivieri M, Donati MB, de Gaetano G, Iacoviello L, Bonanni A

Int J Public Health. 2012;57:589-97. 


\title{
Mass media information and adherence to Mediterranean diet: results from the Moli-sani study
}

\author{
Marialaura Bonaccio - Augusto Di Castelnuovo - Simona Costanzo • \\ Francesca De Lucia - Marco Olivieri - Maria Benedetta Donati · \\ Giovanni de Gaetano $\cdot$ Licia Iacoviello $\cdot$ Americo Bonanni
}

Received: 14 July 2011/Revised: 11 November 2011/Accepted: 6 December 2011/Published online: 21 December 2011

(C) Swiss School of Public Health 2011

\begin{abstract}
Objective To investigate the association between mass media information, dietary habits and risk factors for cardiovascular disease in an Italian adult population.

Methods Subsample of 1,132 subjects (mean age $53 \pm$ $10,50 \%$ men) enrolled in the Moli-sani Project, a population-based cohort study. A specific questionnaire on exposure to information from various media sources was elaborated, validated, and administered. A mass media exposure score was obtained from principal component analysis of ten items of media exposure. Dietary habits were assessed based on eating patterns obtained from principal component analysis of 45 food groups derived from the EPIC food frequency questionnaire and by the Mediterranean score.

Results In a multivariable general linear regression analysis including age, sex, social status, physical activity, C-reactive protein, total calories intake, three dietary patterns or Mediterranean score, higher media exposure was
\end{abstract}

On behalf of the Moli-sani Project Investigators. Moli-sani Project Investigators are listed in the Appendix 1.

M. Bonaccio - F. De Lucia - A. Bonanni

Science Communication Unit, Fondazione di Ricerca e Cura

"Giovanni Paolo II", 86100 Campobasso, Italy

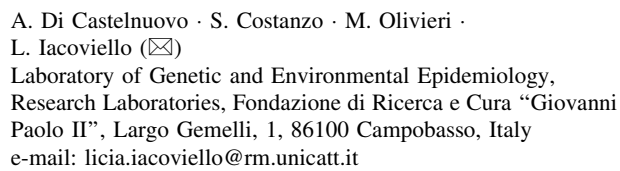

M. B. Donati · G. de Gaetano

Research Laboratories, Fondazione di Ricerca e Cura

"Giovanni Paolo II", 86100 Campobasso, Italy positively associated with adherence to a Mediterraneanlike eating pattern $(P=0.0018)$ as well as to the Mediterranean score $(P=0.0005)$.

Conclusions Exposure to mass media information is significantly associated with greater adherence to both Mediterranean diet and Mediterranean-like eating pattern, an association that public health strategies should take into account.

Keywords Mass media exposure - Mediterranean diet . Cardiovascular risk factors

\section{Introduction}

It is widely recognized that exposure to mass media sources can deeply influence people's behavior, affecting both lifestyles and health. Previous studies have linked Television viewing (TV) to adverse cardiovascular outcomes and obesity in adults (Cleland et al. 2008; Wijndaele et al. 2010; Tucker and Bagwell 1991; Tucker and Friedman 1989), children and adolescents (Jackson et al. 2009). In addition, TV has been strongly associated both with metabolic syndrome (Mark and Janssen 2008; Chang et al. 2008) and elevated cardiovascular risk, and was found to be a predictor of cardiovascular and all-cause mortality risk (Wijndaele et al. 2011; Dunstan et al. 2010). In the US, as well as all over the Western world, TV rates were dramatically increased during the past decades becoming one of the main leisure-time activities.

The majority of the studies conducted so far consider mass media exposure as a measure of sedentary activity rather than a behavior-conditioning source (Hu et al. 2003). Yet other Authors (Redelmeier and Stanbrook 2003) suggested that association between prolonged TV watching 
and risk of obesity may be ascribed to TV content rather than to reduced exercise. The latter hypothesis is quite difficult to prove as TV is just one of the mass media information offerings, besides newspaper and magazine reading and Internet surfing. Thus, little evidence is available on the role of mass media information in promoting behavioral models.

Although the exposure to mass media messages - conceived as a more complete indicator than simply TV watching or surfing the Web-is reportedly associated with either unhealthy food choices (Cleland et al. 2008) and behaviors, evidence is still weak on its association with eating pattern and resulting health outcomes. Evaluating how and to what extent exposure to media information might affect behavior, mainly healthy food choice, might help defining new strategies in prevention of cardiovascular disease.

The aim of the present study was to investigate the effect of mass media information exposure per se, not just as a mere indicator of physical inactivity, on several parameters of cardiovascular risk with particular regard to dietary habits.

\section{Methods}

Study population

The Moli-sani Project is a population-based cohort study which randomly recruited 24,325 citizens of Molise, a region placed between Central and Southern Italy. The study enrolled men and women aged $\geq 35$ years, randomly recruited from subjects included in the city-hall registries of Molise (Iacoviello et al. 2007). Exclusion criteria were pregnancy, disturbances in understanding/willing processes, ongoing poly-traumas or coma, and refusal to sign the informed consent; $30 \%$ of subjects refused to participate; these were generally older and had a higher prevalence of cardiovascular disease.

\section{Dietary information}

The validated Italian EPIC food frequency questionnaire (FFQ) was used to determine food intake (Pala et al. 2003; Pisani et al. 1997). The questionnaire, computerized with tailor-made software, allowed us to interview participants in an interactive way, including illustrations of sample dishes of definite sizes or by reference to standard portion sizes. To simplify interpretation of data and to minimize within-person variations in intakes of individual foods, 188 food items were classified into 45 predefined food groups on the basis of similarity in nutrient characteristics or culinary usage.
Data collection

Body mass index (BMI) was calculated as $\mathrm{kg} / \mathrm{m}^{2}$. Waist circumferences were measured according to the $\mathrm{NIH}$, Heart, Lung, and Blood guidelines (Janssen et al. 2002). Blood pressure was measured by an automatic device (OMRON-HEM-705CP) three times on the non-dominant arm and the average of the last two values was taken as the BP. Hypertension, diabetes, and dyslipidemia were defined as self-reported health professional-diagnosis and antihypertensive, anti-diabetics or lipid-lowering medication use. Socio-economic status was defined as a score based on eight variables (income, education, job, housing, ratio between the number of live-in partners and the number of rooms (both current and in the childhood) and availability of hot water at home in the childhood); the highest the score, the highest the level of socio-economic status. Physical activity was assessed by a structured questionnaire (24 questions on working time, leisure time, and sport participation) and expressed as daily energy expenditure in metabolic equivalent task-hours (MET/d). The risk of fatal cardiovascular events at 10 years was calculated using the CUORE risk equation (http://www.cuore.iss.it/).

Metabolic syndrome (MetS) was defined according to Adult Treatment Panel III criteria (NCEP 2001). Serum lipids and glucose were assayed by enzymatic reaction methods using an automatic analyzer (ILab 350, Instrumentation laboratory (IL), Milan, Italy). LDL-cholesterol was calculated according to Friedewald. High sensitivity C-reactive protein (CRP) was measured in fresh serum, by a latex particle-enhanced immunoturbidimetric assay (IL Coagulation Systems on ACL9000). Inter- and intraday CV were 5.5 and $4.17 \%$, respectively.

\section{Mass media exposure questionnaire}

A questionnaire on mass media information exposure was developed by our Science Communication Unit. The questionnaire included ten questions on (a) newspaper, magazine and specialized press reading frequency; (b) whether subjects feel inclined to receive information on health and prevention from a list of different sources, and (c) whether they use the Internet to visit press agency websites or for reasons other than checking their own mail. We did not take into account time spent sitting watching $\mathrm{TV}$, since we do not believe it might provide any clue on quality information, while participants were asked how often they use to watch TV-news. The description and sorting of the items are illustrated in Table 1. The questionnaire was administered to a sub-sample of the Molisani Project cohort.

All subjects $(n=1,132)$ recruited in the Moli-sani study from May 2009 to April 2010 (end of the whole Moli-sani 
Table 1 Factor loadings of the Mass Media exposure information score empirically derived by PFA in 959 men and women from the Moli-sani Project (Italy, 2011, Moli-sani Project)

\begin{tabular}{|c|c|c|}
\hline Questions & $\begin{array}{l}\text { Possible answers } \\
\text { (one answer only) }\end{array}$ & $\begin{array}{l}\text { Factor } \\
\text { loading }\end{array}$ \\
\hline $\begin{array}{l}\text { How often do you watch TV } \\
\text { news? }\end{array}$ & $\begin{array}{l}\text { Many times a day } \\
\text { Every day } \\
\text { Some days a week } \\
\text { Some days a month } \\
\text { Never or almost never }\end{array}$ & 0.57 \\
\hline $\begin{array}{l}\text { How often do you buy newspapers } \\
\text { per week? }\end{array}$ & $\begin{array}{l}\text { Daily } \\
4-5 \text { times } \\
\text { A couple of times } \\
\text { Never or almost never }\end{array}$ & 0.52 \\
\hline $\begin{array}{l}\text { How often do you read newspapers } \\
\text { not bought directly by you (bar, } \\
\text { doctor, dentist) }\end{array}$ & $\begin{array}{l}\text { Daily } \\
4-5 \text { times } \\
\text { A couple of times } \\
\text { Never or almost never }\end{array}$ & 0.47 \\
\hline $\begin{array}{l}\text { How often do you buy weekly } \\
\text { newspapers in a month? }\end{array}$ & $\begin{array}{l}\text { Once a week } \\
\text { A couple of times } \\
\text { Never or almost never }\end{array}$ & 0.47 \\
\hline $\begin{array}{l}\text { How often do you read weekly } \\
\text { newspapers not bought directly } \\
\text { by you in a month? (bar, doctor, } \\
\text { dentist) }\end{array}$ & $\begin{array}{l}\text { Once a week } \\
\text { A couple of times } \\
\text { Never or almost never }\end{array}$ & 0.44 \\
\hline $\begin{array}{l}\text { How often do you buy monthly } \\
\text { magazines in a year? }\end{array}$ & $\begin{array}{l}\text { Once a month } \\
\text { Less than } 4 \text { times per } \\
\text { year } \\
\text { Never or almost never }\end{array}$ & 0.41 \\
\hline $\begin{array}{l}\text { How often do you read monthly } \\
\text { magazines not bought directly } \\
\text { by you in a year? }\end{array}$ & $\begin{array}{l}\text { Once a month } \\
\text { Less than } 4 \text { times per } \\
\text { year } \\
\text { Never or almost never }\end{array}$ & 0.39 \\
\hline $\begin{array}{l}\text { Have you ever received } \\
\text { information on health and } \\
\text { prevention? }\end{array}$ & $\begin{array}{l}\text { Yes } \\
\text { No }\end{array}$ & 0.36 \\
\hline $\begin{array}{l}\text { Do you read newspapers on line } \\
\text { when surfing the Internet? }\end{array}$ & $\begin{array}{l}\text { Yes } \\
\text { No }\end{array}$ & 0.23 \\
\hline $\begin{array}{l}\text { Do you read press agency on line } \\
\text { when surfing the Internet? }\end{array}$ & $\begin{array}{l}\text { Yes } \\
\text { No }\end{array}$ & 0.11 \\
\hline
\end{tabular}

Project recruitment) were asked to fill the self-questionnaire; participants reporting missing values for media exposure were excluded. The final study sample was 959 subjects. The 173 subjects who were excluded from the analysis were comparable for sex distribution but were older $(P<0.0001)$ and had a lower social status $(P<0.0001)$ in comparison with the 959 subjects who were included in the analysis. It is likely that the questionnaire on mass media information exposure-which was self-administered-was too articulated to be completed by older people and those having lower social status because they might find the questionnaire, largely addressing modern technologies, too far away from their daily experience, and refrained from completing it properly. Yet the 959 analyzed subjects were comparable with the whole Moli-sani Project population in terms of sex distribution, social status, cardiovascular disease, and metabolic syndrome prevalence, BMI, cardiovascular risk and dietary patterns, whereas mean age was only slightly lower $(52.8 \pm 9.6$ vs. $55.6 \pm 11.9)$

To establish validity, test-retest method was used. The questionnaire was administered twice, with a 2-week separation, to a group $(n=27)$ of researchers at the Catholic University of Campobasso. Cronbach's alpha was used to estimate internal consistency reliability of questionnaire.

Statistical analysis

The mass media exposure score was obtained using principal component analysis (PCA). The purpose of PCA is to derive a small number of components that can account for the variability found in a relatively large number of measures. This procedure, called data reduction, is typically performed when a researcher does not want to include all of the original measures in analyses, but still wants to work with the information that they contain. PCA was conducted on the correlation matrix of the ten items in the mass media exposure (predictors) and determines linear functions of predictors (named factors) that explain as much predictor variation as possible. In determining the number of factors to retain in PCA we used the criterions of an eigenvalue $>1.0$, the scree test and the interpretability of the final solution (Kim and Mueller 1978). The correlations between each extracted factor and predictors are called factor loadings. Each subject was attributed, for each pattern, a factor score, calculated by summing all the predictors, each weighted by factor loadings. The application of the PCA to our database clearly indicates the existence of a single factor, whose loadings are illustrated in Table 1. Ranking of the factor in tertiles allowed to classify subjects in three different levels of exposition as light, moderate, and heavy.

Food consumption patterns were generated using PCA conducted on the correlation matrix of the 45 food groups (Centritto et al. 2009). Three main factors emerged with PCA, in agreement with previous findings in the same population (Centritto et al. 2009). The first pattern, named "Olive Oil and Vegetables", was characterized by high positive loadings of olive oil, vegetables, legumes, soups, fruits, and fish. The second pattern, named "Pasta and Meat", was characterized by high positive loadings of pasta, cooked tomatoes, red meat, animal fats and alcoholic beverages, and negative loadings of breakfast cereals, and yogurt. The "Eggs and Sweets" pattern was characterized by high positive loadings of eggs, margarines, processed meat, sugar, and sweets Table 5 in Appendix 2. 
We evaluated the adherence to Mediterranean diet using the Mediterranean diet score (MDS) elaborated by Trichopoulou et al. (2003) which was obtained by assigning a value of 0 or 1 to each of nine indicated components with the use of the sex-specific median as the cut-off. The total Mediterranean- diet score ranged from 0 , which indicates the minimal adherence to the traditional Mediterranean diet, to 9, namely the maximal adherence.

CRP was transformed into natural logarithm to reduce positive skewness, but data were reported untransformed for clarity. Values for continuous variables are mean $\pm \mathrm{SD}$.

The potential predictors tested for association with tertiles of the score of media exposure included sociodemographic variables (age, sex, smoking habit, and social status), serum lipid concentrations (total-, HDL, and LDLcholesterol, and triglycerides), systolic and diastolic blood pressure, blood glucose, BMI, waist to hip ratio, physical activity (expressed as MET/d), cardiovascular risk score, and $\mathrm{C}$-reactive protein. We also considered the association between mass media information exposure and two different definitions of metabolic syndrome.

Generalized linear models (PROC GENMOD in SAS) were used for testing the association of mass media exposure classification (considered as the dependent variable) with potential predictors. The multivariable models were built up as follows: (a) simple univariable analysis was used to identify variables associated with light, moderate, and heavy mass media exposure at the level $P<0.10$ adjusted for age and sex; (b) the variables identified in step a) were inserted in a full model all together. The data analysis was generated using SAS/STAT software, Version 9.1.3 of the SAS System for Windows@2009. SAS Institute Inc. and SAS are registered trademarks of SAS Institute Inc., Cary, NC, USA.

\section{Results}

\section{Validation}

Mass media exposure score was not different between test and retest groups (paired $t$ test $P=0.40$ ) and the two scores were highly correlated $(r=0.84, P<0.0001)$. The reliability of test for items used in media exposure score was 0.66 (as measured by Cronbach's Alpha, standardized).

Mass media information exposure and population characteristics

Table 2 shows the characteristics of the sub-sample of the Moli-sani Project population who completed the mass media information exposure questionnaire.
In univariable analysis people heavily exposed to information showed a significantly higher social status $(P<0.0001)$, lower level of physical activity $(P=0.014)$, and reduced levels of CRP $(P=0.031)$ (Table 2).

In the multivariable analysis controlled for age and sex, heavy mass media exposure remained inversely associated with physical activity $(P=0.0063)$ and positively linked to lower levels of CRP $(P=0.04)$ and higher social status $(P<0.0001)$ (Table 2).

Association between dietary patterns and mass media information exposure

The heavy mass media information exposure group showed a higher adherence to the pattern "Olive Oil and Vegetables" both in age/sex adjusted model $(P=0.0002)$ and in the multivariate model $(P=0.0018)$ which included social status, daily energy intake, physical activity, PFA pattern "Pasta and Meat", PFA pattern "Eggs and Sweets", CRP (Table 2). Data are corroborated by the trend observed in the heavily exposed group which shows a significantly lower adherence to the PFA pattern "Pasta and Meat" compared with the light-moderate exposed groups, either in the univariate model $(P=0.39)$ or in the sex-age adjusted model $(P=0.035)$ (Table 2). Similar results were found when social status was replaced by educational level in multivariable model ( $P=0.0027$ and $P=0.034$ for "Olive oil and vegetables" and "Pasta and Meat" patterns, respectively).

In regard to MDS, subjects in the heavy mass media information exposure group reported higher score than those in the light and moderate categories both in the sexage adjusted model $(P=0.0008)$ and in the two multivariable models $(P=0.0005$ with social status and $P=0.0008$ with adjustment for education) suggesting that the more people are exposed to information, the more they follow the Mediterranean diet. Yet the difference between heavy and light mass media exposure in Mediterranean score was less than one point (Table 2). Moreover, subjects more exposed to information had higher consumption of fruits and fish $(P=0.0031$ and $P=0.0007$, respectively, in multivariable analysis) and lower intakes of animal fats $(P=0.029$ in multivariable analysis) (Table 2).

\section{Stratification for social status}

To further investigate the role of social status and education, we performed additional analyses stratified for these variables. After stratifying by social-status, the same trends recorded in general population were observed (Table 3). Subjects in high, medium, or low social-status categories showed an increasing adherence to both Mediterraneanlike eating pattern and MDS according to mass media information exposure (Table 3). 
Table 2 Score of media exposure obtained from principal component analysis of $n=10$ items (Italy, 2011, Moli-sani Project)

\begin{tabular}{|c|c|c|c|c|c|c|c|}
\hline \multirow[t]{2}{*}{ Population characteristics } & \multirow{2}{*}{$\begin{array}{l}\text { All } \\
(N=959)\end{array}$} & \multicolumn{3}{|c|}{ Categories of mass media information exposure } & \multicolumn{3}{|l|}{$P$ value } \\
\hline & & $\begin{array}{l}\text { Light } \\
(n=319)\end{array}$ & $\begin{array}{l}\text { Moderate } \\
(n=320)\end{array}$ & $\begin{array}{l}\text { Heavy } \\
(n=320)\end{array}$ & Univariable & $\begin{array}{l}\text { Sex/ } \\
\text { age adj }\end{array}$ & Multivariable* \\
\hline Age (years) & $52.8(9.6)$ & $53.1(10.3)$ & $52.3(9.3)$ & $52.9(9.2)$ & $<0.0001$ & - & 0.10 \\
\hline Female $n,(\%)$ & $480(50)$ & $187(58.6)$ & $156(48.7)$ & $137(42.8)$ & 0.0003 & - & $<0.0001$ \\
\hline Male $n,(\%)$ & $479(50)$ & $132(41.4)$ & $164(51.2)$ & $183(57.2)$ & & & \\
\hline Smokers $n,(\%)$ & & & & & 0.039 & 0.11 & \\
\hline Never & $402(41.9)$ & $154(48.3)$ & $115(35.9)$ & $133(41.6)$ & & & \\
\hline Current & $263(27.4)$ & $78(24.4)$ & $98(30.6)$ & $87(27.2)$ & & & \\
\hline Former & $294(30.7)$ & $87(27.3)$ & $107(33.4)$ & $100(31.2)$ & & & \\
\hline Socio-economic status & $7.4(2.35)$ & $6.58(2.05)$ & $7.41(2.28)$ & $8.12(2.45)$ & $<0.0001$ & $<0.0001$ & $<0.0001$ \\
\hline Physical activity (MET-h/day) & $43.8(8.1)$ & $44.8(8.7)$ & $43.8(8.2)$ & $42.8(6.5)$ & 0.014 & 0.0063 & 0.22 \\
\hline BMI $\left(\mathrm{kg} / \mathrm{m}^{2}\right)$ & $27.9(4.7)$ & $28.1(4.8)$ & $27.8(4.7)$ & $27.9(4.6)$ & 0.75 & 0.72 & \\
\hline WH-ratio & $0.89(0.08)$ & $0.89(0.08)$ & $0.89(0.08)$ & $0.89(0.08)$ & 0.17 & 0.96 & \\
\hline Systolic blood pressure $(\mathrm{mmHg})$ & $134.4(18.8)$ & $134.2(18.4)$ & $135.3(19.4)$ & $133.7(18.8)$ & 0.80 & 0.47 & \\
\hline Diastolic blood pressure (mmHg) & $81.7(9.3)$ & $81.8(9.2)$ & $82.2(9.5)$ & $81.2(9.1)$ & 0.47 & 0.32 & \\
\hline Metabolic syndrome (ATPIII) $n,(\%)$ & $240(25.2)$ & $86(27.1)$ & $79(24.8)$ & $75(23.7)$ & 0.59 & 0.58 & \\
\hline Metabolic syndrome (IDF) $n,(\%)$ & $352(36.9)$ & $121(37.9)$ & $114(35.7)$ & $117(36.9)$ & 0.85 & 0.91 & \\
\hline CVD risk & $4.3(5.6)$ & $4.1(4.9)$ & $4.6(6.3)$ & $4.2(5.3)$ & 0.061 & 0.32 & \\
\hline Total cholesterol (mg/dL) & $207.7(44.8)$ & $209.6(45.3)$ & $205.5(45.7)$ & $207.9(43.5)$ & 0.42 & 0.51 & \\
\hline HDL cholesterol (mg/dL) & $55.8(15.2)$ & $57.4(15.8)$ & $54.6(14.3)$ & $56.4(15.5)$ & 0.064 & 0.20 & \\
\hline LDL cholesterol (mg/dL) & $126.9(36.9)$ & $128.2(38.3)$ & $125.9(37.6)$ & $126.5(34.8)$ & 0.65 & 0.73 & \\
\hline Triglycerides (mg/dL) & $125.7(82)$ & $124.1(74.1)$ & $127.1(76.6)$ & $125.8(94.1)$ & 0.41 & 0.89 & \\
\hline Blood glucose $(\mathrm{mg} / \mathrm{dL})$ & $100.8(30.6)$ & $99.4(25.5)$ & $100.8(34.5)$ & $102.1(31.02)$ & 0.53 & 0.64 & \\
\hline CRP (mg/L) & $2.28(2.7)$ & $2.45(2.78)$ & $2.30(2.80)$ & $2.06(2.57)$ & 0.031 & 0.04 & 0.16 \\
\hline Energy intake (kcal/day) & $2,084(596)$ & $2,066(651)$ & $2,061(555)$ & $2,121(575)$ & 0.056 & 0.32 & 0.36 \\
\hline Alcohol intake (g/day) & $14.3(19.6)$ & $14.1(21.8)$ & $13.8(18.7)$ & $15.03(18.2)$ & 0.73 & 0.34 & \\
\hline $\begin{array}{l}\text { Dietary pattern } 1 \text { (Olive Oil } \\
\text { and Vegetables) }\end{array}$ & $-0.012(0.80)$ & $-0.11(0.82)$ & $-0.04(0.74)$ & $0.11(0.82)$ & 0.0014 & 0.0002 & $0.0018 * *$ \\
\hline Dietary pattern 2 (Pasta and Meat) & $0.11(0.85)$ & $0.13(0.89)$ & $0.14(0.84)$ & $0.055(0.83)$ & 0.39 & 0.035 & $0.068 * *$ \\
\hline Dietary pattern 3 (Eggs and Sweets) & $0.15(0.83)$ & $0.12(0.91)$ & $0.13(0.79)$ & $0.19(0.78)$ & 0.41 & 0.52 & $0.73 * *$ \\
\hline Mediterranean Medscore & $4.37(1.61)$ & $4.09(1.62)$ & $4.42(1.64)$ & $4.61(1.54)$ & 0.0003 & 0.0008 & $0.0005 * * *$ \\
\hline Fruits (g/day) & $323.2(175.8)$ & 296.7 (165.6) & $329.3(190.3)$ & $343.3(167.1)$ & 0.0008 & 0.0024 & 0.0031 \\
\hline Fish (g/day) & $23.14(17.5)$ & $19.5(15.6)$ & $24.3(18.2)$ & $25.5(18)$ & $<0.0001$ & $<0.0001$ & 0.0007 \\
\hline Animal fats (g/day) & $1.71(1.52)$ & $1.85(1.45)$ & $1.70(1.63)$ & $1.56(1.48)$ & 0.43 & 0.04 & 0.029 \\
\hline
\end{tabular}

* Adjusted for sex, age, total energy intake, social status, physical activity and C-reactive protein

** Adjusted for sex, age, total energy intake, physical activity, C-reactive protein and the other two dietary patterns

*** Adjusted for sex, age, total energy intake, physical activity and C-reactive protein

Both in the high and low educated group, adherence to Mediterranean diet (both Mediterranean-like eating pattern and Mediterranean score) followed the gradient of mass media information exposure (Table 4).

\section{Discussion}

Exposure to information delivered by mass media was associated with a greater adherence to both Mediterranean diet and Mediterranean diet-like eating pattern in a representative large sample of a general adult Italian population. We measured global information offered by mass media by a specific score to provide a reliable assessment of how people are exposed to media message on a regular basis.

We avoided to consider just the time subjects were engaged in activities such as sitting and watching TV or standing at the desk while surfing the Internet: these measures are useful to assess inactivity (Hu et al. 2003; Gao et al. 2007) but are less effective in determining whether 
Table 3 Mass media information exposure and dietary patterns according to stratification by socio-economic status (SES) (Italy, 2011, Molisani Project)

\begin{tabular}{|c|c|c|c|c|c|}
\hline \multirow[t]{2}{*}{ Low SES } & \multicolumn{3}{|c|}{ Categories of mass media information exposure } & \multicolumn{2}{|l|}{$P$ value } \\
\hline & Light $(n=153)$ & Moderate $(n=110)$ & Heavy $(n=78)$ & Univariable & Multivariable \\
\hline Dietary pattern 1 (Olive Oil and Vegetables) & $-0.14(0.81)$ & $-0.01(0.73)$ & $0.05(0.69)$ & 0.18 & $0.26^{*}$ \\
\hline Dietary pattern 2 (Pasta and Meat) & $0.15(0.95)$ & $0.18(0.88)$ & $0.22(0.81)$ & 0.85 & $0.22 *$ \\
\hline Dietary pattern 3 (Eggs and Sweets) & $0.09(0.82)$ & $0.17(0.81)$ & $0.19(0.82)$ & 0.58 & $0.51 *$ \\
\hline Mediterranean Medscore & $4.09(1.68)$ & $4.62(1.62)$ & $4.72(1.48)$ & 0.0055 & $0.010 * *$ \\
\hline \multirow[t]{2}{*}{ Medium SES } & \multicolumn{3}{|c|}{ Categories of mass media information exposure } & \multicolumn{2}{|l|}{$P$ value } \\
\hline & Light $(n=105)$ & Moderate $(n=106)$ & Heavy $(n=94)$ & Univariable & Multivariable \\
\hline Dietary pattern 1 (Olive Oil and Vegetables) & $-0.13(0.81)$ & $-0.13(0.73)$ & $0.17(0.69)$ & 0.013 & $0.03^{*}$ \\
\hline Dietary pattern 2 (Pasta and Meat) & $0.17(0.86)$ & $0.28(0.86)$ & $0.11(0.77)$ & 0.33 & $0.04 *$ \\
\hline Dietary pattern 3 (Eggs and Sweets) & $0.16(1.04)$ & $0.15(0.82)$ & $0.30(0.75)$ & 0.44 & $0.79 *$ \\
\hline Mediterranean Medscore & $4.00(1.54)$ & $4.19(1.56)$ & $4.52(1.54)$ & 0.059 & $0.04 * *$ \\
\hline \multirow[t]{2}{*}{ High SES } & \multicolumn{3}{|c|}{ Categories of mass media information exposure } & \multicolumn{2}{|l|}{$P$ value } \\
\hline & Light $(n=58)$ & Moderate $(n=103)$ & Heavy $(n=148)$ & Univariable & Multivariable \\
\hline Dietary pattern 1 (Olive Oil and Vegetables) & $-0.01(0.82)$ & $0.02(0.73)$ & $0.11(0.69)$ & 0.52 & $0.12 *$ \\
\hline Dietary pattern 2 (Pasta and Meat) & $0.03(0.77)$ & $-0.06(0.75)$ & $-0.06(0.86)$ & 0.72 & $0.78^{*}$ \\
\hline Dietary pattern 3 (Eggs and Sweets) & $0.10(0.89)$ & $0.08(0.76)$ & $0.14(0.78)$ & 0.81 & $0.16^{*}$ \\
\hline Mediterranean Medscore & $4.29(1.60)$ & $4.43(1.72)$ & $4.60(1.58)$ & 0.43 & $0.48 * *$ \\
\hline
\end{tabular}

* Adjusted for sex, age, total energy intake, physical activity, C-reactive protein and the other two dietary patterns

** Adjusted for sex, age, total energy intake, physical activity and C-reactive protein

Table 4 Mass media information exposure and dietary patterns according to stratification by education (Italy, 2011, Moli-sani Project)

\begin{tabular}{|c|c|c|c|c|c|}
\hline \multirow[t]{2}{*}{ Higher education $(n=482)$} & \multicolumn{3}{|c|}{ Categories of mass media information exposure } & \multicolumn{2}{|l|}{$P$ value } \\
\hline & Light $(n=103)$ & Moderate $(n=172)$ & Heavy $(n=206)$ & Univariable & Multivariable \\
\hline Dietary pattern 1 (Olive Oil and Vegetables) & $-0.083(0.80)$ & $-0.020(0.74)$ & $0.14(0.86)$ & 0.04 & $0.015^{*}$ \\
\hline Dietary pattern 2 (Pasta and Meat) & $0.0073(0.82)$ & $0.082(0.84)$ & $-0.0013(0.85)$ & 0.6 & $0.22 *$ \\
\hline Dietary pattern 3 (Eggs and Sweets) & $0.17(0.83)$ & $0.069(0.77)$ & $0.19(0.76)$ & 0.29 & $0.54 *$ \\
\hline Mediterranean Medscore & 3.95 & 4.37 & 4.62 & 0.0020 & $0.015 * *$ \\
\hline \multirow[t]{2}{*}{ Lower education $(n=476)$} & \multicolumn{3}{|c|}{ Categories of mass media information exposure } & \multicolumn{2}{|l|}{$P$ value } \\
\hline & Light $(n=213)$ & Moderate $(n=146)$ & Heavy $(n=114)$ & Univariable & Multivariable** \\
\hline Dietary pattern 1 (Olive Oil and Vegetables) & $-0.12(0.83)$ & $-0.07(0.74)$ & $0.07(0.75)$ & 0.10 & $0.09 *$ \\
\hline Dietary pattern 2 (Pasta and Meat) & $0.20(0.91)$ & $0.20(0.85)$ & $0.16(0.79)$ & 0.89 & $0.06^{*}$ \\
\hline Dietary pattern 3 (Eggs and Sweets) & $0.092(0.95)$ & $0.20(0.83)$ & $0.22(0.82)$ & 0.35 & $0.76^{*}$ \\
\hline Mediterranean Medscore & $4.17(1.71)$ & $4.47(1.63)$ & $4.59(1.56)$ & 0.06 & $0.04 * *$ \\
\hline
\end{tabular}

* Adjusted for sex, age, total energy intake, physical activity, C-reactive protein and the other two dietary patterns

** Adjusted for sex, age, total energy intake, physical activity and C-reactive protein

people do receive real information or just entertainment (Redelmeier and Stanbrook 2003).

Whereas previous studies had shown that massive exposition to TV is linked to adverse cardiovascular outcomes such as increased overweight, obesity (Cleland et al. 2008; Wijndaele et al. 2010; Tucker and Bagwell 1991;
Tucker and Friedman 1989), and metabolic syndrome (Mark and Janssen 2008; Chang et al. 2008), the present study, where an alternative measure of mass media usage was performed, mainly focused on information, and provides different results showing that the more the subjects get informed the more they stick to healthy eating habits. 
On the basis of our findings, we speculate that information resulting from a balanced mix of different sources could positively influence people's food choices, leading them to combine healthy foods in their diet (Reger et al. 1999; Freisling et al. 2010) independently from other possible confounding factors such as social status.

In Italy, this could be partially ascribed to the growing attention mass media information is paying to nutrition, wellbeing, and good lifestyle in general. Newspapers, magazines, websites, radio, and TV broadcasting news are giving more and more space to health-related issues regarding nutrition, physical activity, quitting smoking, and management of body weight (Hornik and Kelly 2007). Mediterranean diet, even before being listed by UNESCO among world's cultural heritage (http://www.unesco.org/ culture/ich/en/RL/00394; Bonaccio et al. 2011), has always received impressive media coverage on Italian press, generating a high level of interest towards this topic and a sense of national pride for culinary traditions typical of our Country. This may help explaining the linkage between high mass media information exposure and a greater adherence to Mediterranean-like eating pattern.

Limitations of the study

A major limitation of the present study is its cross-sectional nature which is a limit of epidemiological studies of this kind. Nevertheless, this type of investigation is useful to set new hypotheses to be tested in future prospective studies. Second, caution is needed in extending the results presented here to larger contexts since data were collected in a region located between Central and Southern Italy, Mediterranean by tradition and culture (Iacoviello et al. 2007). Yet, the main characteristics of our sample are comparable to those of the Italian Cardiovascular Epidemiological Observatory (Giampaoli et al. 2009), a large survey including random samples of the general population all over Italy; therefore our sample could be considered representative of the Italian population.

A third limit is related to the fact that due to the structure of the questionnaire, mainly addressing issues related to modern technologies, older people might have found it too difficult to complete. In fact the population included in the analysis was almost 3 years younger than the whole Moli-sani project sample and this was the only remarkable difference. Nevertheless, further studies should address this issue to include also older people in the analysis.

Fourth, our score of mass media information exposure was unable to define the type and quality of information subjects were exposed to, but only how much they were exposed to mass media information.

Potential confounding factors could be represented by social status and education which are potentially linked both to a better information level and adherence to healthy dietary pattern (Darmon and Drewnowski 2008). However, adjustment and stratification for social status index or educational levels did not influence the association between mass media exposure and adherence to Mediterranean-like eating pattern and MDS.

\section{Conclusions}

In conclusion, mass media do not necessarily represent a threat to public health, as suggested by previous studies, but they can play a positive role in promoting healthy behaviors through propagating balanced information. A role that public health strategies should take into account. In order to gain maximum advantage from mass media delivered messages, future research should extend knowledge on an issue not addressed by this study, namely analyzing the content quality of information sources.

Ethical issues The Moli-sani Project was approved by the Ethics Committee of the Catholic University, Rome. Participants signed an informed consent form before taking part in the study.

Acknowledgments The Moli-sani Project was partially supported by research Grants from Pfizer Foundation (Rome, Italy) and the Italian Ministry of University and Research (MIUR, Rome, Italy)Programma Triennale di Ricerca, Decreto no.1588. Neither sponsor had any role in study design, collection, analysis, and interpretation of data; in the writing of the report; and in the decision to submit the article for publication.

Conflict of interest All authors had full access to all of the data in the study and take responsibility for the integrity of the data and the accuracy of the data analysis. None of the authors had a personal or financial conflict of interest.

\section{Appendix 1}

\section{Moli-sani Project Investigators}

Chairperson: Licia Iacoviello.

Steering Committee: Maria Benedetta Donati and Giovanni de Gaetano (Chairpersons), Simona Giampaoli (Istituto Superiore di Sanità, Roma).

Bio-ethics Committee: Jos Vermylen (University of Leuven, Belgio), Chairman, Ignacio De Paula Carrasco (Pontificia Academia Pro Vita, Roma), Enrico Garaci (Istituto Superiore di Sanità, Roma).

Event adjudicating Committee: Deodato Assanelli (Università di Brescia), Francesco Alessandrini (UCSC, Campobasso), Vincenzo Centritto (Campobasso), Paola Muti (Istituto Nazionale Tumori Regina Elena IRCCS, Roma), Holger Schunemann (McMaster University Health Sciences Centre, Canada), Pasquale Spagnuolo (Ospedale 
San Timoteo, Termoli), Dante Staniscia (Ospedale San Timoteo, Termoli), Sergio Storti (UCSC, Campobasso).

Scientific and Organizing Secretariat: Francesco Zito (Coordinator), Americo Bonanni, Chiara Cerletti, Amalia De Curtis, Augusto Di Castelnuovo, Licia Iacoviello, Antonio Mascioli, Marco Olivieri.

Data Management and Analysis: Augusto Di Castelnuovo (Coordinator), Antonella Arcari, Floriana Centritto (till December 2008), Simona Costanzo, Romina di Giuseppe, Francesco Gianfagna, Iolanda Santimone.

Informatics: Marco Olivieri (Coordinator), Maurizio Giacci, Antonella Padulo (till September 2008), Dario Petraroia (till September 2007).

Research Biobank and Biochemical Analyses: Amalia De Curtis (Coordinator), Sara Magnacca, Federico Marracino (till June 2009), Maria Spinelli, Christian Silvestri (till December 2007), Cristina Vallese (till September 2008);

Genetics: Daniela Cugino, Monica de Gaetano (till October 2008), Mirella Graziano (till July 2009), Iolanda Santimone, Maria Carmela Latella (till December 2008), Gianni Quacquaruccio (till December 2007);

Communication: Americo Bonanni (Coordinator), Marialaura Bonaccio, Francesca De Lucia.

Moli-family Project: Branislav Vohnout (Coordinator) (till December 2008), Francesco Gianfagna, Andrea Havranova (till July 2008), Antonella Cutrone (till October 2007);

Recruitment staff (2005-2010): Franco Zito (General Coordinator), Secretariat: Mariarosaria Persichillo (Coordinator), Angelita Verna, Maura Di Lillo (till March 2009), Irene Di Stefano (till March 2008), Blood sampling: Agostino Panichella, Antonio Rinaldo Vizzarri, Branislav Vohnout (till December 2008), Agnieszka Pampuch (till August 2007); Spirometry: Antonella Arcari (Coordinator), Daniela Barbato (till July 2009), Francesca Bracone, Simona Costanzo, Carmine Di Giorgio (till September 2008), Sara Magnacca, Simona Panebianco (till December 2008), Antonello Chiovitti (till March 2008), Federico Marracino (till December 2007), Sergio Caccamo (till August 2006), Vanesa Caruso (till May 2006); Electrocardiograms: Livia Rago (Coordinator), Daniela Cugino, Francesco Zito, Alessandra Ferri (till October 2008), Concetta Castaldi (till September 2008), Marcella Mignogna (till September 2008); Tomasz Guszcz (till January 2007), Questionnaires: Romina di Giuseppe, (Coordinator), Paola Barisciano, Lorena Buonaccorsi (till December 2008), Floriana Centritto (till December 2008), Francesca De Lucia, Francesca Fanelli (till January 2009), Iolanda Santimone, Anna Sciarretta, Maura Di Lillo (till March 2009), Isabella Sorella (till September 2008), Irene Di Stefano (till March 2008), Emanuela Plescia (till December 2007), Alessandra Molinaro (till December 2006), Christiana Cavone (till September 2005);
Call Center: Giovanna Galuppo (till June 2009), Maura Di Lillo (till March 2009), Concetta Castaldi (till September 2008), Dolores D'Angelo (till May 2008), Rosanna Ramacciato (till May 2008).

\section{Appendix 2}

See Table 5.

Table 5 Food grouping used in the dietary pattern analyses

\begin{tabular}{|c|c|}
\hline $\begin{array}{l}\text { Foods or food } \\
\text { groups }\end{array}$ & Food items \\
\hline Potatoes & Potatoes \\
\hline $\begin{array}{l}\text { Cooked } \\
\text { vegetables }\end{array}$ & $\begin{array}{l}\text { Leafy vegetables, root vegetables, cabbages, onion, } \\
\text { carrots, mushrooms, egg plants, artichokes, } \\
\text { sweet peppers, spinach, pumpkins, canned } \\
\text { vegetables in oil, picked vegetables }\end{array}$ \\
\hline Raw vegetables & Raw leafy vegetables, raw tomatoes \\
\hline $\begin{array}{l}\text { Tomatoes } \\
\text { (cooked) }\end{array}$ & Tomato sauces, tomatoes \\
\hline Legumes & Beans, lentils, peas, chick peas \\
\hline Fruit & $\begin{array}{l}\text { Apples, pears, kiwi, bananas, grapes, peaches, } \\
\text { apricots, oranges, tangerines, plums, } \\
\text { strawberries, melon, khaki, figs, cherries }\end{array}$ \\
\hline $\begin{array}{l}\text { Nuts and dried } \\
\text { fruit }\end{array}$ & $\begin{array}{l}\text { Peanuts, almonds, hazelnuts, walnuts, dried figs, } \\
\text { dried dates, prune }\end{array}$ \\
\hline Olives & Olives \\
\hline Milk & Milk \\
\hline Yogurt & Yogurt \\
\hline Fresh cheese & $\begin{array}{l}\text { Mozzarella, ricotta cheese, taleggio cheese, } \\
\text { gorgonzola cheese, melted cheese slices, other } \\
\text { soft cream cheese }\end{array}$ \\
\hline Seasoned cheese & $\begin{array}{l}\text { Fontina cheese, emmenthal, gruyere, parmesan, } \\
\text { caciocavallo cheese, other seasoned cheese }\end{array}$ \\
\hline $\begin{array}{l}\text { Pasta and other } \\
\text { grains }\end{array}$ & Pasta, yellow maize meal \\
\hline Rice & Rice \\
\hline Bread & White bread, bread with oil and other bread \\
\hline $\begin{array}{l}\text { Crisp bread, } \\
\text { rusks }\end{array}$ & Breads sticks, crisp bread \\
\hline $\begin{array}{r}\text { Breakfast } \\
\text { cereals }\end{array}$ & Breakfast cereals \\
\hline Salty biscuits & Crackers \\
\hline Red meat & Beef, pork, lamb, horse, game, veal, other meats \\
\hline White meat & Chicken, turkey, rabbit \\
\hline Processed meat & Sausages, ham, bologna sausage, dried beef, salami \\
\hline Offals & Liver, offals \\
\hline Canned fish & Canned tuna fish and other fish \\
\hline $\begin{array}{l}\text { Crustaceans, } \\
\text { molluscs }\end{array}$ & Crustaceans, molluscs \\
\hline Fish & Other fish \\
\hline Egg & Eggs \\
\hline
\end{tabular}


Table 5 continued

\begin{tabular}{|c|c|}
\hline $\begin{array}{l}\text { Foods or food } \\
\text { groups }\end{array}$ & Food items \\
\hline Vegetables oils & Seed oils (except olive oils) \\
\hline Olive oil & Olive oil \\
\hline Butter & Butter \\
\hline Margarines & Margarines \\
\hline Animal fats & Visible fat from meat, poultry skin, fat from ham \\
\hline Sugar \& sweets & $\begin{array}{l}\text { Sugar, honey, cakes, ice cream, confections, pastry, } \\
\text { pudding }\end{array}$ \\
\hline Fruit juices & Orange juice, grapefruit juices, other fruit juices \\
\hline Soft drinks & Soft drinks \\
\hline Coffee & Coffee \\
\hline Tea & Tea \\
\hline Other sauces & Dressing sauces for pasta other than tomato sauce \\
\hline Mayonnaises & Mayonnaises \\
\hline Soups & Vegetable soups \\
\hline Bouillon & Meat and stock-cube broth \\
\hline Snacks & Vegetable quiche \\
\hline Pizza & Pizza \\
\hline Wine & Red wine, rosé wine, white wine \\
\hline Spirits & Alcoholic beverages other than wine or beer \\
\hline Beer & Beer \\
\hline
\end{tabular}

\section{References}

Bonaccio M, Iacoviello L and de Gaetano G (2011) The Mediterranean diet: the reasons for a success. Thromb Res [Epub ahead of print]

Centritto F, Iacoviello L, di Giuseppe R, De Curtis A, Costanzo S, Zito F, Grioni S, Sieri S, Donati MB, de Gaetano G, Di Castelnuovo A, Moli-sani Investigators (2009) Dietary patterns, cardiovascular risk factors and C-reactive protein in a healthy Italian population. Nutr Metab Cardiovasc Dis 19:697-706

Chang PC, Li TC, Wu MT, Liu CS, Li CI, Chen CC, Lin WY, Yang SY, Lin CC (2008) Association between television viewing and the risk of metabolic syndrome in a community-based population. BMC Public Health 8:193

Cleland VJ, Schmidt MD, Dwyer T, Venn AJ (2008) Television viewing and abdominal obesity in young adults: is the association mediated by food and beverage consumption during viewing time or reduced leisure-time physical activity? Am J Clin Nutr 87:1148-1155

Darmon N, Drewnowski A (2008) Does social class predict diet quality? Am J Clin Nutr 87:1107-1117

Dunstan DW, Barr EL, Healy GN, Salmon J, Shaw JE, Balkau B, Magliano DJ, Cameron AJ, Zimmet PZ, Owen N (2010) Television viewing time and mortality: the Australian Diabetes, Obesity and Lifestyle Study (Aus Diab). Circulation 121:384-391

Executive Summary of The Third Report of The National Cholesterol Education Program (NCEP) Expert Panel on Detection (2001) Evaluation, and Treatment of High Blood Cholesterol in Adults (Adult Treatment Panel III) JAMA 285:2486-2497

Freisling H, Haas K, Elmadfa I (2010) Mass media nutrition information sources and associations with fruit and vegetable consumption among adolescents. Public Health Nutr 13:269-275
Gao X, Nelson ME, Tucker KL (2007) Television viewing is associated with prevalence of metabolic syndrome in Hispanic elders. Diabetes Care 30:694-700

Giampaoli S, Rielli R, Dematté L, Donfrancesco C, Lo Noce C, Dima F, De Sanctis Caiola P, Ciccarelli P, De Rosa M, Addis A, Palmieri L (2009) The Italian observatory of cardiovascular risk: the CUORE project experience [abstract]. Circulation 119:139

Hornik R, Kelly B (2007) Communication and diet: an overview of experience and principles. J Nutr Educ Behav 239(2 Suppl):S5-S12

$\mathrm{Hu}$ FB, Li TY, Colditz GA, Willett WC, Manson JE (2003) Television watching and other sedentary behaviors in relation to risk of obesity and type 2 diabetes mellitus in women. JAMA 289:1785-1791

Iacoviello L, Bonanni A, Costanzo S, De Curtis A, Di Castelnuovo A, Olivieri M, Zito F, Donati MB, de Gaetano G, on behalf of the Moli-sani Project Investigators (2007) The Moli-sani Project, a randomized, prospective cohort study in the Molise region in Italy; design, rationale and objectives. Italian J Public Health 4:110-118

ISTAT (2006) L'uso dei media e del cellulare in Italia. Indagine multiscopo sulle famiglie "I cittadini e il tempo libero". http:// www.istat.it/dati/catalogo/20080429_00/testointegrale20080429. pdf. Accessed 27 May 2011

Jackson DM, Djafarian K, Stewart J, Speakman JR (2009) Increased television viewing is associated with elevated body fatness but not with lower total energy expenditure in children. Am J Clin Nutr 89:1031-1036

Janssen I, Katzmarzyk PT, Ross R (2002) Body mass index, waist circumference, and health risk: evidence in support of current National Institutes of Health guidelines. Arch Intern Med 162:2074-2079

Kim JO, Mueller CW (1978) Factor analysis: statistical method and practical issues. Sage Publications, Thousand Oaks

Mark AE, Janssen I (2008) Relationship between screen time and metabolic syndrome in adolescents. J Public Health (Oxf) 30:153-160

Pala V, Sieri S, Palli D et al (2003) Diet in the Italian EPIC cohorts: presentation of data and methodological issues. Tumori 89:594-607

Pisani P, Faggiano F, Krogh V, Palli D, Vineis P, Berrino F (1997) Relative validity and reproducibility of a food frequency dietary questionnaire for use in the Italian EPIC centres. Int J Epidemiol 26(Suppl. 1):S152-S160

Redelmeier DA, Stanbrook MB (2003) Television viewing and risk of obesity. JAMA 290:332 author reply 332

Reger B, Wootan MG, Booth-Butterfield S (1999) Using mass media to promote healthy eating: a community-based demonstration project. Prev Med 29:414-421

Special Eurobarometer 293 (2008) E-communications Household Survey. Version current June 2008. http://ec.europa.eu/public_ opinion/archives/ebs/ebs_293_full_en.pdf. Accessed 28 October 2010

Trichopoulou A, Costacou T, Bamia C, Trichopoulos D (2003) Adherence to a Mediterranean diet and survival in a Greek population. N Engl J Med 348:2599-2608

Tucker LA, Bagwell M (1991) Television viewing and obesity in adult females. Am J Public Health 81:908-911

Tucker LA, Friedman GM (1989) Television viewing and obesity in adult males. Am J Public Health 79:516-518

Wijndaele K, Healy GN, Dunstan DW, Barnett AG, Salmon J, Shaw JE, Zimmet PZ, Owen N (2010) Increased cardiometabolic risk is associated with increased TV viewing time. Med Sci Sports Exerc 42:1511-1518

Wijndaele K, Brage S, Besson H, Khaw KT, Sharp SJ, Luben R, Wareham NJ, Ekelund U (2011) Television viewing time independently predicts all-cause and cardiovascular mortality: the EPIC Norfolk Study. Int J Epidemiol 40:150-159 


\section{CHAPTER 8.}

Nutrition knowledge is associated with higher adherence to Mediterranean diet and lower prevalence of obesity. Results from the Moli-sani study

Bonaccio M, Di Castelnuovo A, Costanzo S, De Lucia F, Olivieri M, Donati MB, de Gaetano G, Iacoviello L, Bonanni A

Appetite. 2013;68:139-46. 
Research report

\title{
Nutrition knowledge is associated with higher adherence to Mediterranean diet and lower prevalence of obesity. Results from the Moli-sani study ${ }^{\text {th }}$
}

\author{
Marialaura Bonaccio a , Augusto Di Castelnuovo a , Simona Costanzo a, Francesca De Lucia a , Marco Olivieri ${ }^{a}$, \\ Maria Benedetta Donati ${ }^{\mathrm{b}}$, Giovanni de Gaetano ${ }^{\mathrm{b}, \mathrm{c}}$, Licia Iacoviello ${ }^{\mathrm{a}, *}$, Americo Bonanni ${ }^{\mathrm{a}, \mathrm{c}}$, \\ On behalf of the Moli-sani Project Investigators ${ }^{1}$
}

${ }^{a}$ Laboratory of Genetic and Environmental Epidemiology, Research Laboratories, Fondazione di Ricerca e Cura "Giovanni Paolo II", Largo Gemelli 1, 86100 Campobasso, Italy

${ }^{\mathrm{b}}$ Research Laboratories, Fondazione di Ricerca e Cura "Giovanni Paolo II", Largo Gemelli 1, 86100 Campobasso, Italy

${ }^{\mathrm{C}}$ EPICOMED Research Srl, Corso Mazzini 2, 86100 Campobasso, Italy

\section{A R T I C L E I N F O}

\section{Article history:}

Received 7 February 2013

Received in revised form 19 March 2013

Available online 7 May 2013

\section{Keywords:}

Nutrition knowledge

Mediterranean diet

Obesity

Socioeconomic status

\begin{abstract}
A B S T R A C T
A Mediterranean dietary pattern has been associated with reducing the risk of cardiovascular and chronic disease. The aim of this study was to evaluate the role of nutrition knowledge in determining possible differences among dietary patterns in a general population from a Mediterranean region. We conducted a cross-sectional study on a subsample of 744 subjects enrolled in the population-based cohort of the Moli-sani Project. A 92-item questionnaire on nutrition knowledge was elaborated, validated and administered. Dietary information were obtained from the EPIC food frequency questionnaire and adherence to a Mediterranean dietary pattern was evaluated both by the a priori Greek Mediterranean diet score and the a posteriori approach obtained by principal component analysis. Nutrition knowledge was significantly associated with higher adherence to a Mediterranean dietary pattern. The odds of having higher adherence to a Mediterranean dietary pattern increased with greater nutrition knowledge. The odds ratio of being obese significantly decreased with increasing nutrition knowledge levels. The results showed that nutrition knowledge was significantly associated with higher adherence to a Mediterranean dietary pattern and with lower prevalence of obesity in a Southern Italian region with Mediterranean diet tradition independently from education and other socioeconomic factors.
\end{abstract}

2013 Elsevier Ltd. All rights reserved.

\section{Introduction}

Mediterranean diet (MD) is one of the most effective eating patterns associated with the reduction of the risk of cardiovascular and other chronic disease (Bonaccio, Iacoviello, de Gaetano, \& On Behalf of the Moli-sani Investigators, 2012; Sofi, Abbate, Gensini, \& Casini, 2010; Tangney et al., 2011). Nevertheless, adherence to MD has dramatically decreased in recent years and this trend is even more evident in the southern European and Northern African countries, where the Mediterranean eating pattern originated

\footnotetext{
Acknowledgements: (M.B., A.B., L.I. designed the research; F.D.L., M.B., M.O managed data collection; M.B., A.D.C., S.C. analyzed the data; M.B., A.B. wrote the paper, M.B.D., G.d.G., L.I. critically reviewed the manuscript). All authors had full access to all of the data in the study and take responsibility for the integrity of the data and the accuracy of the data analysis. Conflict of interest: The authors have no conflict of interest to disclose.

* Corresponding author.

E-mail address: licia.iacoviello@moli-sani.org (L. Jacoviello).

${ }^{1}$ Moli-sani Project Investigators are listed in Appendix B.
}

(Mehio Sibai et al., 2010). Studies performed in a southern region of Italy, which is supposed to have a Mediterranean-like dietary tradition, highlighted that the Mediterranean pattern is becoming dramatically unpopular among the youngest while it remains a widespread choice just for the elderly (di Giuseppe et al., 2008). Increasing trends in cardiovascular risk factors, primarily obesity and hypertension, have been linked to changing patterns in lifestyle behaviors across Southern Italy and other Mediterranean countries (Laccetti et al., 2013).

In addition, social and cultural changes have been ascribed a leading role in determining the shifting of dietary habits towards other types of diet such as the Western diet model, which is rich in refined grains, saturated fats, sugars, red meat and processed meat. Several investigations highlighted a conditioning role of socioeconomic status (SES) showing that persons in the lower SES groups are more likely to exhibit poorer diets (Darmon \& Drewnowski, 2008). A major role is played by income which turned out to be a strong obstacle towards healthy eating patterns in particular to MD (Bonaccio, Bonanni et al., 2012). Low education also 
has been linked to poor adherence to Mediterranean-like eating patterns. The Social Cognitive Theory (Bandura, 1991) suggests that to perform a particular behavior a person has to know what the behavior is and how to perform it. In the case of diet, according to this theory, a person has to know what a good diet is before asking him/her to follow it properly.

That is why more recently, research focused on the possible relationship between diet quality and other social and cultural factors, such as nutrition knowledge and beliefs which are considered to be important factors explaining variations in food choices (Sharma, Gernand, \& Day, 2008; Wardle, Parmenter, \& Waller, 2000). Nutrition knowledge has also been shown to act as an effect modifier (Beydoun \& Wang, 2008) and to be a mediator between socioeconomic position and diet quality (McLeod, Campbell, \& Hesketh, 2011). The aim of this study was to test the association between nutrition knowledge (NK) and adherence to MD in an adult population living in a central-southern region of Italy, with Mediterranean dietary traditions.

\section{Methods}

\section{Study population}

The Moli-sani Project is a population-based cohort study on 24,325 citizens of Molise, a region placed between Central and Southern Italy. The study enrolled men and women aged $\geqslant 35$ years, randomly recruited from subjects included in the city-hall registries of Molise (Iacoviello et al., 2007). Exclusion criteria were pregnancy, disturbances in understanding/willing processes, ongoing poly-traumas or coma, refusal to sign the informed consent; $30 \%$ of subjects refused to participate; these were generally older and had a higher prevalence of cardiovascular disease or cancer. This study was conducted in the framework of a sub-study of the Moli-sani Project aimed at investigating the association between nutrition knowledge, mass media media exposure (Bonaccio, Di Castelnuovo et al., 2012) and dietary habits and lifestyle. In 2009, we elaborated an additional questionnaire on nutrition knowledge and mass media exposure. Once validated, the questionnaire was administered to participants recruited from May 2009 to April 2010 ( $n=1571$ ), when the Moli-sani study officially closed the enrolment phase, with participation rate equal to $72 \%$. Finally, 1132 subjects completed the questionnaire.

\section{Dietary information}

The validated Italian EPIC food frequency questionnaire (FFQ) was used to assess food intake (Pala et al., 2003; Pisani et al., 1997). The questionnaire, computerized with tailor-made software, allowed interviewing participants in an interactive way, including illustrations of sample dishes of definite sizes or by reference to standard portion sizes. To simplify interpretation of data and to minimize within-person variations in intakes of individual foods, 188 food items were classified into 45 predefined food groups on the basis of similar nutrient characteristics or culinary usage (Appendix A).

Food consumption patterns were generated by using Principal Components Analysis (PCA) conducted on the correlation matrix of 45 food groups (Centritto et al., 2009). Three main factors emerged, in agreement with previous findings in the same population (Centritto et al., 2009). The first pattern, identified as "Olive Oil and Vegetables", was characterized by high positive loadings for olive oil, vegetables, legumes, soups, fruits and fish. The second pattern, named "Pasta and Meat", was characterized by high positive loadings for pasta, cooked tomatoes, red meat, animal fats and alcoholic beverages, and negative loadings of breakfast cereals and yogurt. The "Eggs and Sweets" pattern was characterized by high positive loadings for eggs, margarines, processed meat and sugar and sweets. This "a posteriori" approach, based on the foods actually consumed by participants, allows to overcome the limitations of the "a priori" scores, not accounting for correlations in foods consumption.

We evaluated the adherence to the Mediterranean diet by using the Mediterranean diet score (MDS) elaborated by Trichopoulou, Costacou, Bamia, and Trichopoulos (2003). Scoring was based on the intake of the following nine items: vegetables, legumes, fruit and nuts, dairy products, cereals, meat and meat products, fish, alcohol, and the ratio of monounsaturated:saturated fat. For most items, consumption above the study median received 1 point; all other intakes received 0 points. For dairy products, meat and meat products, consumption below the median received 1 point. Medians are gender specific. For ethanol, men who consumed 10$50 \mathrm{~g} /$ day and women who consumed 5-25 g/day received 1 point; otherwise, the score was 0 . The possible scores ranged between 0 and 9 , the latter reflecting the maximal adherence.

\section{Data collection}

Body mass index (BMI) was calculated as $\mathrm{kg} / \mathrm{m}^{2}$. Waist circumferences were measured according to the $\mathrm{NIH}$, Heart, Lung, and Blood guidelines (Janssen, Katzmarzyk, \& Ross, 2002). Blood pressure was measured by an automatic device (OMRON-HEM$705 \mathrm{CP}$ ) three times on the non-dominant arm, with the patient lying down for about $5 \mathrm{~min}$. Hypertension was defined as systolic $\mathrm{BP} \geqslant 140 \mathrm{mmHg}$ or diastolic $\mathrm{BP} \geqslant 90 \mathrm{mmHg}$, or using pharmacological treatment. Diabetes was defined as blood glucose $>126 \mathrm{mg} / \mathrm{dl}$ or using pharmacological treatment. Physical activity was assessed by a structured questionnaire (24 questions on working time, leisure time and sport participation) and expressed as daily energy expenditure in metabolic equivalent task-hours (MET/d). Obesity was defined as BMI $>30$.

\section{Socioeconomic variables}

Household net income categories were considered as low ( $\leqslant 25,000$ euro/year), medium ( $>25,000$ and $\leqslant 40,000$ euro/year) and high ( $>40,000$ euro/year). We recorded a high percentage (30\%) of non-respondent subjects who refused to declare or did not know their household income. Yet, such a large non-respondent group is very common in this type of investigations, especially among women and the elderly (Kaplan \& Keil, 1993).

Education level was considered as low ( $\leqslant 8$ years) and higher ( $>8$ years). Socioeconomic status (SES) was expressed as a score based on five variables: dwelling ownership and ratio between the number of rooms and number of living-in family members (rooms per person), both currently and during childhood, and availability of hot water at home during childhood. The five components were dichotomized according to the median value, and a score of one was attributed to the category supposed to be marker of higher social status in comparison with the opposite category: thus we assigned a score of 1 to people living in a house with living-in family members/room density $>0.6$ or dwelling ownership or with availability of hot water and a score 0 to people with living-in family members/room density $\leqslant 0.6$, no dwelling ownership or with unavailability of hot water. We recorded missing values for SES for $6.3 \%$ of the subjects.

The SES score did not include income and education. Marital status was considered as married or live-in partner versus others (divorced, unmarried and widower). 


\section{Nutrition knowledge questionnaire}

Nutrition knowledge was a section of a wider questionnaire on attitudes, information and behaviors in relation to nutrition and health developed by our Science Communication Unit. A section on mass media exposure was previously used to evaluate its relationship with dietary habits (Bonaccio, Di Castelnuovo, et al., 2012).

Items for establishing knowledge of food content were extracted from the questionnaire elaborated by Parmenter and Wardle (1999). Classes of foods were adapted to Italian culinary tradition.

The self-completed questionnaire included 92 multiple-choice questions aimed at evaluating the knowledge of participants on several aspects of nutrition knowledge. The questions covered different areas of nutrition knowledge: awareness of experts' recommendations (11 items), food content and calories (68 items), relationship between diet and diseases (13 items).

We assigned one point for each correct answer, 0 point for each "do not know" answer and a penalization of one point to incorrect answers.

Subjects with missing values $>32$ (corresponding to $35 \%$ of answers) were excluded from the analysis $(n=386)$ together with those for whom no information on diet were available $(n=2)$. The final study sample was 744 subjects. The nutrition knowledge score ranged from -9 to 72 . Subjects were divided into tertiles based on these scores and categorized as low, medium and high.

Subjects excluded from the analysis were comparable for sex distribution but were older $(52.1 \pm 9.4$ versus $58.5 \pm 11.0$; $p$ value $<0.0001$ ), had lower educational level, income and socioeconomic status ( $p$ value $<0.0001$ ) in comparison with the final sample. Reasons for exclusion may lie in the fact that the questionnaire on nutrition knowledge - which was self-administered - could seem not easily understandable to older people and those having lower social status.

Comparison between the whole Moli-sani cohort $(n=24,325)$ and the analyzed subsample $(n=744)$ showed homogeneity for sex $(p=0.20)$ but the subsample was younger $(55 \pm 11.8$ versus $52 \pm 9.4 ; p<0.0001)$ and had higher social status $(p<0.0001)$, income $(p=0.001)$, and education $(p=0.01)$.

To establish validity, test-retest method was used. The questionnaire was administered twice, with a 2 -weeks separation, to a group ( $n=28$ ) of researchers at the Fondazione di Ricerca e Cura Giovanni Paolo II of Campobasso. Nutrition knowledge scores between test and retest groups were highly correlated (Spearman correlation coefficient $r=0.71, p<0.0001$ ).

\section{Statistical analysis}

Values for continuous variables are means \pm Standard Deviation.

The potential predictors tested for association with tertiles of the score of nutrition knowledge included socio-demographic variables (age, sex, BMI, income, educational level, socioeconomic status), smoking habit and physical activity (expressed as MET/d). Multivariable analysis of variance for continuous or categorical variables was used for testing the associations of general characteristics or adherence to Mediterranean diet scores or other dietary pattern (considered as the dependent variables) with NK categories. By using multivariable logistic regression analysis, odds ratio (ORs) with corresponding 95\% confidence intervals (95\% CI) were calculated to quantify the association of NK categories with obesity and adherence to Mediterranean diet. High adherence to MD, as stated by the Medscore, was defined when the score was $>4$ points whereas low adherence when the score was $\leqslant 4$ points. A specific category was created either for income or SES in order to consider the missing values in the multivariate analyses.

The data analysis was generated using SAS/STAT software, Version 9.1.3 of the SAS System for Windows $\odot 2009$. SAS Institute Inc. and SAS are registered trademarks of SAS Institute Inc., Cary, NC, USA.

\section{Results}

Mean value of nutrition knowledge score for the whole sample was 37.2. The main characteristics of the sample according to NK categories are shown in Table 1 . People in the highest tertile of NK were prevalently women (56.6\%), had higher educational level ( $p$ for trend $<.0001)$, income $(p=0.0006)$ and socioeconomic status $(p=0.03)$. BMI and obesity prevalence were higher in the lowest category (28.6 \pm 4.5 and $34.6 \%$, respectively) and lower in the highest category of NK $(27.1 \pm 4.5$ and $21.9 \%, p=0.005$ and $p=0.01$ respectively, Table 1$)$.

The association between NK and adherence to Mediterranean diet or to other dietary patterns is shown in Table 2. Multivariate analysis adjusted for possible confounding factors (age, sex, energy intake, BMI, physical activity, educational level, income, socioeconomic status and smoking) shows that subjects in the highest tertile of NK were more likely to follow the Mediterranean diet either according to the Medscore ( $p$ for trend $=0.01$ ) or to the Olive oil and vegetable pattern ( $p$ for trend $=0.001$ ).

Stratified analysis by education levels ( $\leqslant 8$ years and $>8$ years) was conducted to better address the possible confounding effect of education in the association between NK and adherence to MDP. After stratifying by education, the same trends recorded in the whole population were observed (Table 3 ). Subjects in the uppermost education level showed an increasing adherence to MDS ( $p$ for trend $=0.02$ ) and to the Olive oil and vegetable pattern ( $p$ for trend $=0.01$ ) according to NK categories (Table 3 ). In the lower educated group statistical significance was reached only for the Olive oil and vegetable pattern for the basic model ( $p$ for trend $=0.02$ ) and was borderline significant for the fully adjusted model $(p$ for trend $=0.05$ ).

We then divided the Mediterranean score into two categories to get both a low (score from 0 to 4 ) and a high adherence (score > 4). In multivariable logistic regression model controlling for age, sex and daily energy intake, the odds of having a higher adherence to MD increased according to NK levels (Table 4). People in the highest NK group had $52 \%$ statistically significant higher probability to be in the uppermost category of Mediterranean diet adherence than those in the lowest NK group (Table 4). Once other variables were included in the model (physical activity, BMI, educational level, income, socioeconomic status, marital status and smoking), respondents in the highest tertile of nutrition knowledge were still more likely to be in the highest category of MD adherence compared to those in the lowest tertile $(\mathrm{OR}=1.62,95 \% \mathrm{CI}: 1.09-2.40)$ for highest versus lowest NK group.

We also evaluated the association of nutrition knowledge and obesity. Odds ratio of being obese decreased according to NK levels ( $\mathrm{OR}=0.61,95 \% \mathrm{CI}: 0.40-0.95$ for highest versus lowest NK group) in the fully adjusted model (Table 4). After including the Medscore or the Olive oil and vegetables pattern in the model, the association between obesity and NK was not modified.

\section{Discussion}

The rising epidemic of obesity and increasing prevalence of chronic disease worldwide have been partially ascribed to unhealthy eating behaviors. MD is associated with a reduction in the risk of developing chronic disease, neurodegenerative disorders 
Table 1

Main characteristics of the sample according to Nutrition knowledge levels.

\begin{tabular}{|c|c|c|c|c|c|}
\hline & \multicolumn{5}{|c|}{ Nutrition knowledge levels } \\
\hline & All $(n=744)$ & $\begin{array}{l}\text { Low }(-9 \text { to } 32) \\
(n=254,34.1 \%)\end{array}$ & $\begin{array}{l}\text { Medium }(33-44) \\
(n=248,33.3 \%)\end{array}$ & $\begin{array}{l}\text { High }(45-72) \\
(n=242,32.5 \%)\end{array}$ & $p$ Value ${ }^{*}$ \\
\hline Age (years, means $\pm \mathrm{SD}$ ) & $52.1(9.4)$ & $53.0(10.4)$ & $51.5(9.0)$ & $51.7(8.5)$ & 0.18 \\
\hline Sex (males, \%) & $50.3 \%$ & $55.9 \%$ & $51.2 \%$ & $43.4 \%$ & 0.02 \\
\hline Total physical activity (MET-h/day, means \pm SD) & $43.8(7.9)$ & $45.0(8.4)$ & $43.7(8.5)$ & $42.7(6.5)$ & 0.003 \\
\hline Leisure time physical activity (MET-h/day, means \pm SD) & $1.8(1.7)$ & $1.8(1.9)$ & $1.6(1.5)$ & $1.9(1.8)$ & 0.63 \\
\hline Working time physical activity (MET-h/day, means \pm SD) & $15.5(10.4)$ & $16.9(10.6)$ & $14.1(11.5)$ & $12.8(8.5)$ & 0.02 \\
\hline Educational level $(\%)$ ( $\geqslant 8$ years) & $52.0 \%$ & $30.3 \%$ & $56.8 \%$ & $69.8 \%$ & $<.0001$ \\
\hline Income (\%) ${ }^{\mathrm{a}}$ & & & & & 0.0006 \\
\hline Low & $39.0 \%$ & $41.7 \%$ & $43.1 \%$ & $31.8 \%$ & \\
\hline Medium & $22.6 \%$ & $16.9 \%$ & $23.8 \%$ & $27.3 \%$ & \\
\hline High & $7.7 \%$ & $5.1 \%$ & $7.7 \%$ & $10.3 \%$ & \\
\hline Socioeconomic status (\%) ${ }^{\mathrm{a}}$ & & & & & 0.03 \\
\hline Low & $24.3 \%$ & $27.6 \%$ & $23.8 \%$ & $21.5 \%$ & \\
\hline Medium & $28.5 \%$ & $31.9 \%$ & $27.8 \%$ & $25.6 \%$ & \\
\hline High & $40.9 \%$ & $31.9 \%$ & $42.7 \%$ & $48.3 \%$ & \\
\hline Marital status (\%) (married or living-in partners) & $85.2 \%$ & $87.8 \%$ & $86.3 \%$ & $81.4 \%$ & 0.14 \\
\hline \multicolumn{6}{|l|}{ Smokers (\%) } \\
\hline Never & $40.7 \%$ & $33.9 \%$ & $43.9 \%$ & $44.6 \%$ & \\
\hline Current & $27.7 \%$ & $35.0 \%$ & $23.4 \%$ & $24.4 \%$ & 0.01 \\
\hline Former & $31.6 \%$ & $31.1 \%$ & $32.7 \%$ & $31.0 \%$ & \\
\hline BMI $\left(\mathrm{kg} / \mathrm{m}^{2}\right)$ & $27.9(4.7)$ & $28.6(4.5)$ & $27.9(4.9)$ & $27.1(4.5)$ & 0.005 \\
\hline Obesity (\%) & $28.5 \%$ & $34.6 \%$ & $28.6 \%$ & $21.9 \%$ & 0.01 \\
\hline Hypertension (\%) & $44.6 \%$ & $48.4 \%$ & $43.5 \%$ & $41.7 \%$ & 0.85 \\
\hline Diabetes (\%) & $9.8 \%$ & $9.4 \%$ & $9.3 \%$ & $10.7 \%$ & 0.85 \\
\hline
\end{tabular}

$p$ Value adjusted for sex and age.

${ }^{\text {a }}$ Numbers do not add up to $100 \%$ due to missing values.

Table 2

Adherence to Mediterranean diet and other dietary patterns according to Nutrition knowledge levels.

\begin{tabular}{|c|c|c|c|c|c|}
\hline & \multicolumn{5}{|c|}{ Nutrition knowledge levels } \\
\hline & $\begin{array}{l}\text { Low }(-9 \text { to } 32) \\
(n=254,34.1 \%)\end{array}$ & $\begin{array}{l}\text { Medium }(33-44) \\
(n=248,33.3 \%)\end{array}$ & $\begin{array}{l}\text { High }(45-72) \\
(n=242,32.5 \%)\end{array}$ & $\begin{array}{l}p \text { for } \\
\text { trend }^{*}\end{array}$ & $\begin{array}{l}p \text { for trend } \\
\text { Multivariable }\end{array}$ \\
\hline Mediterranean score & $4.19(1.64)$ & $4.44(1.61)$ & $4.55(1.56)$ & 0.01 & 0.01 \\
\hline Olive oil and vegetables pattern & $-0.11(0.95)$ & $-0.04(0.82)$ & $0.16(0.96)$ & 0.0003 & 0.001 \\
\hline Pasta and meat pattern & $0.08(0.93)$ & $-0.01(0.95)$ & $-0.08(0.89)$ & 0.03 & 0.35 \\
\hline Eggs and sweets pattern & $0.03(0.79)$ & $0.03(1.06)$ & $-0.06(0.80)$ & 0.17 & 0.10 \\
\hline Energy intake & $2104(656)$ & $2179(630)$ & $2037(511)$ & 0.20 & 0.73 \\
\hline
\end{tabular}

Means adjusted for age, sex, energy intake.

$p$ for trend value adjusted for age, sex and energy intake.

" $p$ for trend value adjusted for age, sex, energy intake, BMI, physical activity, educational level, income, socioeconomic status, marital status and smoking.

and type 2 diabetes in addition to higher quality of life and lower mortality (Henríquez Sánchez et al., 2012; InterAct Consortium, 2011; Trichopoulou, Bamia, \& Trichopoulos, 2009). Redirecting people's eating habits toward a MD model represents a complex task calling for interventions at different levels of public health.

In our study we found that nutrition knowledge was significantly associated with adherence to a Mediterranean dietary pattern (evaluated either with a priori or a posteriori approach) and with a lower prevalence of obesity.

The importance of nutrition knowledge was particularly marked when results were stratified for education. It is rather reasonable that people with higher degree of education could be more keen to be more aware of the nutritional quality of food and thus to choose high-quality products. Although education is associated with $\mathrm{NK}$, as predictable, it only marginally modifies the association of knowledge with Mediterranean diet.

Similar results were observed in a study on mass media exposure where adherence to a Mediterranean dietary pattern increased with greater exposure to the information delivered by different mass media sources in higher educated people (Bonaccio, Di Castelnuovo, et al., 2012).
Both studies indicate that dietary behaviors are dramatically influenced not only by the environment where people live in, such as peers and family, but also by the quantity and quality of information on specific health-related issues which nowadays is able to reach everybody worldwide.

We also observed that people with higher nutrition knowledge have lower prevalence of obesity. This association allows to suppose that higher nutrition knowledge awareness may lead not only to healthier food choices but also to reduced rates of obesity, although the two effects are not dependent on each other: indeed, differences in obesity rates observed in the different NK categories appear not to be mediated by Mediterranean diet. However, the epidemiological evidence supporting a causal link between Mediterranean diet and body weight is contrasting (Buckland, Bach, \& Serra-Majem, 2008).

Health experts have long been wondering on the effectiveness of health promotion initiatives to improve people's behaviors. The major limit of such initiatives has been ascribed to the gap between research and practice leading to call for further efforts to implement new strategies of intervention (Ory, Jordan, \& Bazzarre, 2002). 
Table 3

Dietary patterns according to Nutrition knowledge levels and stratified by education.

\begin{tabular}{|c|c|c|c|c|c|}
\hline & \multicolumn{5}{|c|}{ Nutrition knowledge levels } \\
\hline & $\begin{array}{l}\text { Low }(-9 \text { to } 32) \\
(n=254,34.1 \%)\end{array}$ & $\begin{array}{l}\text { Medium }(33-44) \\
(n=248,33.3 \%)\end{array}$ & $\begin{array}{l}\text { High }(45-72) \\
(n=242,32.5 \%)\end{array}$ & $\begin{array}{l}p \text { for } \\
\text { trend }\end{array}$ & $\begin{array}{l}p \text { for trend } \\
\text { multivariate }^{* *}\end{array}$ \\
\hline \multicolumn{6}{|l|}{ Higher education ( $n=387)$} \\
\hline Mediterranean diet & $4.00(1.59)$ & $4.37(1.62)$ & $4.51(1.59)$ & 0.02 & 0.02 \\
\hline Olive Oil and vegetables pattern & $-0.09(0.95)$ & $-0.07(0.84)$ & $0.16(0.98)$ & 0.01 & 0.01 \\
\hline Pasta and meat pattern & $-0.01(0.93)$ & $-0.11(0.93)$ & $-0.16(0.88)$ & 0.17 & 0.40 \\
\hline Eggs and sweets pattern & $0.05(0.81)$ & $0.04(1.14)$ & $-0.03(0.82)$ & 0.40 & 0.40 \\
\hline \multicolumn{6}{|l|}{ Lower education $(n=357)$} \\
\hline Mediterranean diet & $4.30(1.67)$ & $4.52(1.60)$ & $4.60(1.48)$ & 0.13 & 0.10 \\
\hline Olive Oil and vegetables pattern & $-0.11(0.96)$ & $0.001(0.80)$ & $0.14(0.92)$ & 0.02 & 0.05 \\
\hline Pasta and meat pattern & $0.13(0.94)$ & $0.12(0.98)$ & $0.08(0.88)$ & 0.71 & 0.57 \\
\hline Eggs and sweets pattern & $0.03(0.78)$ & $0.01(0.97)$ & $-0.13(0.77)$ & 0.11 & 0.12 \\
\hline
\end{tabular}

Means adjusted for sex, age and total energy intake.

" $p$ for trend value adjusted for age, sex and energy intake.

" $p$ for trend value adjusted for age, sex, energy intake, BMI, physical activity, income, socioeconomic status, marital status and smoking.

Table 4

Odds ratios of having high adherence to Mediterranean diet or being obese according to Nutrition knowledge levels.

\begin{tabular}{|c|c|c|c|c|c|c|}
\hline Mediterranean score & Low $(0-4)(n=401,53.9 \%)$ & $\operatorname{High}(>4)(n=343,46.1 \%)$ & $\mathrm{OR}^{\mathrm{a}}$ & $(95 \% \mathrm{CI})$ & $\mathrm{OR}^{\mathrm{b}}$ & $(95 \% \mathrm{CI})$ \\
\hline \multicolumn{7}{|l|}{ Nutrition knowledge } \\
\hline Low $(\%)$ & $36.9 \%$ & $30.9 \%$ & $-1-$ & (Referent) & $-1-$ & (Referent) \\
\hline Medium (\%) & $32.9 \%$ & $33.8 \%$ & 1.22 & $(0.85-1.75)$ & 1.26 & $(0.86-1.84)$ \\
\hline High (\%) & $30.2 \%$ & $35.3 \%$ & $\begin{array}{l}1.52 \\
p \text { for trend } d^{a}=0.023\end{array}$ & $(1.06-2.19)$ & $\begin{array}{l}1.62 \\
p \text { for trend }{ }^{b}=0.016\end{array}$ & $(1.09-2.40)$ \\
\hline Obesity & $\mathrm{BMI} \leqslant 30(n=732,71.5 \%)$ & $\mathrm{BMI}>30(n=212,28.5 \%)$ & $\mathrm{OR}^{\mathrm{a}}$ & $(95 \% \mathrm{CI})$ & $\mathrm{OR}^{\mathrm{b}}$ & $(95 \% \mathrm{Cl})$ \\
\hline \multicolumn{7}{|l|}{ Nutrition knowledge } \\
\hline Low $(\%)$ & $31.2 \%$ & $41.5 \%$ & $-1-$ & (Referent) & $-1-$ & (Referent) \\
\hline Medium (\%) & $33.3 \%$ & $33.5 \%$ & 0.76 & $(0.52-1.11)$ & 0.82 & $(0.55-1.23)$ \\
\hline High (\%) & $35.5 \%$ & $25.0 \%$ & $\begin{array}{l}0.54 \\
p \text { for trend } d^{a}=0.0030\end{array}$ & $(0.36-0.81)$ & $\begin{array}{l}0.61 \\
p \text { for trend } d^{b}=0.029\end{array}$ & $(0.40-0.95)$ \\
\hline
\end{tabular}

${ }^{\text {a }}$ Adjusted for age, sex, energy intake.

b Adjusted for age, sex, energy intake, BMI (not included in the analysis for obesity), physical activity, educational level, income, socioeconomic status, marital status and smoking.

In regard to dietary improvement, the Behavior Change Consortium (Ory et al., 2002), conceived with the task of evaluating effectiveness of novel ways of intervention, remarked that despite there have been some advances in improving dietary behaviors, the majority of Americans did not meet the national dietary guidelines. Among the solutions to bridge the gap between behavior and health strategies, enhancing subjects' knowledge yields good results in terms of healthy attitudes. A study conducted by Powers et al. (2005) indicated that an 8-week nutrition education program on dietary behavior and NK significantly improved dietary behavior and increased $\mathrm{NK}$ in second-grade and third-grade students. Similar positive effects were reached also in intervention studies performed on adult populations discrediting the belief that age is a limiting factor in implementing people's knowledge (Sahyoun, Pratt, \& Anderson, 2004; Bernstein et al., 2002).

Many investigations have explored the association between NK and diet quality finding out that awareness of dietary guidelines, food content and expert's recommendations are useful to people to get healthy eating patterns. These findings led authors to strongly welcome health education programs to make people aware about their dietary choices (Beydoun \& Wang, 2008). It is thus plausible that lack of knowledge can lead people to pursue bad habits in terms of dietary choices and this finds a scientific support in several sociological theories such as the Social Cognitive Theory (Bandura, 1991). A recent survey conducted in Italy by a group of researchers from the University of Bologna (Cevenini, De Sando, Noera, et al., 2011) showed that more than a half of the interviewed people did not know what the term "Mediterranean diet" exactly stands for and there is reason to believe that this lack of awareness might be somehow linked to the progressive abandonment of Mediterranean traditions.
In addition, the importance of improving health awareness emerges from recent evidence showing that knowledge and measures of cognitive ability explained up to $30 \%$ of the educational gradient in health behaviors (Cutler \& Lleras-Muney, 2010), one of the markers of socioeconomic disparities in health.

\section{Limitations of this study}

A major limitation of the present study is due to its cross-sectional nature and shares all the limitations of this study type, especially in regards to causality. However, it could be possible to speculate that higher awareness on health-related issues, such as nutrition knowledge, could lead people to adopt healthier diets due to a greater understanding on risks and benefits related to dietary behaviors. Potential selection biases of the subsample could emerge since the questionnaire on nutrition knowledge was selfadministered and the topic not easily understandable by the elderly or less educated people. Comparison between the whole Moli-sani cohort and the subsample considered in the present study showed a substantial homogeneity for sex, but the sample was younger and had higher social status, income and education. Despite these differences, the analyzed subsample is widely representative for age, social status and education levels, to such an extent to allow the evaluation of the role of these variables.

\section{Conclusions}

Besides the cross-sectional nature of our study, we may speculate that nutrition knowledge somehow influences people's dietary habits independently from other socioeconomic factors. These data 
support the issue of improving people's knowledge on health-related issues as a possible tool for promoting healthier choices, also in terms of dietary habits, independently from other less-modifiable risk factors such as socioeconomic position.

\section{Ethical issues}

The Moli-sani study was approved by the Ethics Committee of the Catholic University of Rome. Participants signed the informed consent before taking part in the study.

\section{Funding}

The enrolment phase of the Moli-sani Project was supported by research grants from Pfizer Foundation (Rome, Italy) and the Italian Ministry of University and Research (MIUR, Rome, Italy) - Programma Triennale di Ricerca, Decreto No. 1588. Neither Pfizer Foundation nor MIUR had any role in study design, collection, analysis, and interpretation of data; in the writing of the report; and in the decision to submit the article for publication.

All Authors are independent from funders.

\section{Appendix A. Food grouping used in the dietary pattern analyses}

\begin{tabular}{|c|c|}
\hline Foods or food groups & Food items \\
\hline Potatoes & Potatoes \\
\hline Cooked vegetables & $\begin{array}{l}\text { Leafy vegetables, root vegetables, cabbages, onion, carrots, mushrooms, egg plants, artichokes, } \\
\text { sweet peppers, spinach, pumpkins, canned vegetables in oil, picked vegetables }\end{array}$ \\
\hline Raw vegetables & Raw leafy vegetables, raw tomatoes \\
\hline Tomatoes (cooked) & Tomato sauces, tomatoes \\
\hline Legumes & Beans, lentils, peas, chick peas \\
\hline Fruit & $\begin{array}{l}\text { Apples, pears, kiwi, bananas, grapes, peaches, apricots, oranges, tangerines, plums, strawberries, } \\
\text { melon, khaki, figs, cherries }\end{array}$ \\
\hline Nuts and dried fruit & Peanuts, almonds, hazelnuts, walnuts, dried figs, dried dates, prune \\
\hline Olives & Olives \\
\hline Milk & Milk \\
\hline Yogurt & Yogurt \\
\hline Fresh cheese & $\begin{array}{l}\text { Mozzarella, ricotta cheese, taleggio cheese, gorgonzola cheese, melted cheese slices, other } \\
\text { soft cream cheese }\end{array}$ \\
\hline Seasoned cheese & Fontina cheese, emmenthal, gruyere, parmesan, caciocavallo cheese, other seasoned cheese \\
\hline Pasta and other grains & Pasta, yellow maize meal \\
\hline Rice & Rice \\
\hline Bread & White bread, bread with oil and other bread \\
\hline Crisp bread, rusks & Breads sticks, crisp bread \\
\hline Breakfast cereals & Breakfast cereals \\
\hline Salty biscuits & Crackers \\
\hline Red meat & Beef, pork, lamb, horse, game, veal, other meats \\
\hline White meat & Chicken, turkey, rabbit \\
\hline Processed meat & Sausages, ham, bologna sausage, dried beef, salami \\
\hline Offals & Liver, offals \\
\hline Canned fish & Canned tuna fish and other fish \\
\hline Crustaceans, molluscs & Crustaceans, molluscs \\
\hline Fish & Other fish \\
\hline Egg & Eggs \\
\hline Vegetables oils & Seed oils (except olive oils) \\
\hline Olive oil & Olive oil \\
\hline Butter & Butter \\
\hline Margarines & Margarines \\
\hline Animal fats & Visible fat from meat, poultry skin, fat from ham \\
\hline Sugar \& sweets & Sugar, honey, cakes, ice cream, confections, pastry, pudding \\
\hline Fruit juices & Orange juice, grapefruit juices, other fruit juices \\
\hline Soft drinks & Soft drinks \\
\hline Coffee & Coffee \\
\hline Tea & Tea \\
\hline Other sauces & Dressing sauces for pasta other than tomato sauce \\
\hline Mayonnaises & Mayonnaises \\
\hline Soups & Vegetable soups \\
\hline Bouillon & Meat and stock-cube broth \\
\hline Snacks & Vegetable quiche \\
\hline Pizza & Pizza \\
\hline Wine & Red wine, rosé wine, white wine \\
\hline Spirits & Alcoholic beverages other than wine or beer \\
\hline Beer & Beer \\
\hline
\end{tabular}


Appendix B. Moli-sani Project Investigators

Steering Committee: Licia Iacoviello, Chairperson, Maria Benedetta Donati and Giovanni de Gaetano (Campobasso,Italy), Simona Giampaoli (Roma, Italy)

Safety and data monitoring Committee: Jos Vermylen (Leuven, Belgio), Chairman, Ignacio De Paula Carrasco (Roma, Italy), Enrico Garaci (Roma, Italy)

Event adjudicating Committee: Deodato Assanelli (Brescia, Italy), Francesco Alessandrini, Vincenzo Centritto and Sergio Storti (Campobasso, Italy)), Paola Muti (Roma, Italy), Holger Schünemann (Hamilton, Ontario, Canada), Pasquale Spagnuolo and Dante Staniscia (Termoli, Italy)

Scientific and organizing secretariat: Francesco Zito (Coordinator, Campobasso and Termoli, Italy), Americo Bonanni, Chiara Cerletti, Amalia De Curtis, Augusto Di Castelnuovo, Licia Iacoviello, Roberto Lorenzet, Antonio Mascioli, Marco Olivieri and Domenico Rotilio (Campobasso, Italy)

Data management and analysis: Augusto Di Castelnuovo, Coordinator, Antonella Arcari, Marialaura Bonaccio, Floriana Centritto (till December 2008), Simona Costanzo, Romina di Giuseppe and Francesco Gianfagna (Campobasso, Italy)

Informatics: Marco Olivieri (Coordinator), Maurizio Giacci, Antonella Padulo (till September 2008) and Dario Petraroia (till September 2007) (Campobasso, Italy)

Biobank and biochemical analyses: Amalia De Curtis (Coordinator), Sara Magnacca, Federico Marracino (till June 2009), Maria Spinelli, Christian Silvestri (till December 2007), and Cristina Vallese (till September 2008) (Campobasso and Termoli, Italy):

Genetics: Daniela Cugino, Monica de Gaetano (till October 2008), Mirella Graziano, Iolanda Santimone, Maria Carmela Latella (till December 2008) and Gianni Quacquaruccio (till December 2007) (Campobasso, Italy)

Communication and Press Office: Americo Bonanni (Coordinator), Marialaura Bonaccio and Francesca De Lucia(Campobasso, Italy):

Moli-family Project: Branislav Vohnout (Coordinator) (till December 2008), Francesco Gianfagna, Andrea Havranova (till July 2008), Antonella Cutrone (till October 2007) (Campobasso, Italy)

Recruitment staff: (Campobasso and Termoli, Italy): Franco Zito (General Coordinator), Secretariat: Mariarosaria Persichillo (Coordinator), Angelita Verna, Maura Di Lillo (till March 2009), Irene Di Stefano (till March 2008), Blood sample: Agostino Pannichella, Antonio Rinaldo Vizzarri, Branislav Vohnout (till December 2008), Agnieszka Pampuch (till August 2007); Spirometry: Antonella Arcari (Coordinator), Daniela Barbato (till July 2009), Francesca Bracone, Simona Costanzo, Carmine Di Giorgio (till September 2008), Sara Magnacca, Simona Panebianco (till December 2008), Antonello Chiovitti (till March 2008), Federico Marracino (till December 2007), Sergio Caccamo (till August 2006), Vanesa Caruso (till May 2006); Electrocardiogram: Livia Rago (Coordinator), Daniela Cugino, Francesco Zito, Alessandra Ferri (till October 2008), Concetta Castaldi (till September 2008), Marcella Mignogna (till September 2008); Tomasz Guszcz (till January 2007), Questionnaires: Romina di Giuseppe, (Coordinator), Paola Barisciano, Lorena Buonaccorsi, Floriana Centritto (till December 2008), Francesca De Lucia, Francesca Fanelli (till January 2009), Iolanda Santimone, Anna Sciarretta, Maura Di Lillo (till March 2009), Isabella Sorella (till September 2008), Irene Di Stefano (till March 2008), Emanuela Plescia (till December 2007), Alessandra Molinaro (till December 2006), and Christiana Cavone (till September 2005), (Campobasso and Termoli, Italy)

Call Center: Giovanna Galuppo (till June 2009), Maura Di Lillo (till March 2009), Concetta Castaldi (till September 2008), Dolores D’Angelo (till May 2008) and Rosanna Ramacciato (till May 2008) (Campobasso, Italy)

\section{References}

Bandura, A. (1991). Social cognitive theory of self-regulation. Organizational Behavior and Human Decision Processes, 50, 248-285.

Bernstein, A., Nelson, M. E., Tucker, K. L., Layne, J., Johnson, E., Nuernberger, A., et al. (2002). A home-based nutrition intervention to increase consumption of fruits, vegetables, and calcium-rich foods in community dwelling elders. Journal of the American Dietetic Association, 102, 1421-1427.

Beydoun, M. A., \& Wang, Y. (2008). Do nutrition knowledge and beliefs modify the association of socio-economic factors and diet quality among US adults? Preventive Medicine, 46, 145-153.

Bonaccio, M., Bonanni, A. E., Di Castelnuovo, A., De Lucia, F., Donati, M. B., de Gaetano, G., et al. (2012). Low income is associated with poor adherence to Mediterranean diet and a higher prevalence of obesity. Cross-sectional results from the Moli-sani study. BMJ Open 19, 2(6). http://dx.doi.org/10.1136/ bmjopen-2012-001685.

Bonaccio, M., Di Castelnuovo, A., Costanzo, S., De Lucia, F., Olivieri, M., Donati, M. B. et al. (2012). C. Mass media information and adherence to Mediterranean diet: results from the Moli-sani study. Int J Public Health, 57, 589-9715.

Bonaccio, M., Iacoviello, L., de Gaetano, G., \& On Behalf of the Moli-sani Investigators (2012). The Mediterranean diet. The reasons for a success. Thrombosis Research, 129, 401-404.

Buckland, G., Bach, A., \& Serra-Majem, L. (2008). Obesity and the Mediterranean diet. A systematic review of observational and intervention studies. Obesity Reviews, 9, 582-593.

Centritto, F., Iacoviello, L., di Giuseppe, R., De Curtis, A., Costanzo, S., Zito, F., et al. (2009). Dietary patterns, cardiovascular risk factors and C-reactive protein in a healthy Italian population. Nutrition Metabolism and Cardiovascular Diseases, 19 697-706.

Cevenini, M., De Sando, V., Noera, G., et al. (2011). Alimentary pyramid and nutritional education knowledge favours Mediterranean diet in selected population groups (Selected Abstracts from the National Congress of the Italian Society for Cardiovascular Prevention (SIPREC), Genova, Italy, 31 March-
2 April 2011) (Report). High Blood Pressure \& Cardiovascular Prevention 18.2 (April 2011, p71(2))

Cutler, D. M., \& Lleras-Muney, A. (2010). Understanding differences in health behaviors by education. Journal of Health Economics, 29, 1-28.

Darmon, N., \& Drewnowski, A. (2008). Does social class predict diet quality? American Journal of Clinical Nutrition, 87, 1107-1117.

di Giuseppe, R., Bonanni, A., Olivieri, M., Di Castelnuovo, A., Donati, M. B., de Gaetano G., et al. (2008). Adherence to Mediterranean diet and anthropometric and metabolic parameters in an observational study in the 'Alto Molise' region. The metabolic parameters in an observational study in the 'Alto Molise' region. The

Henríquez Sánchez, P., Ruano, C., de Irala, J., Ruiz-Canela, M., Martínez-González, M. A., \& Sánchez-Villegas, A. (2012). Adherence to the Mediterranean diet and quality of life in the SUN Project. European Journal of Clinical Nutrition, 66 360-368.

Iacoviello, L., Bonanni, A., Costanzo, S., De Curtis, A., Di Castelnuovo, A., Olivieri, M. et al. (2007). The Moli-sani Project, a randomized, prospective cohort study in the Molise region in Italy; design, rationale and objectives. Italian Journal of Public Health, 4, 110-118.

InterAct Consortium Romaguera, D., Guevara, M., Norat, T., Langenberg, C., Forouhi, N. G., \& Sharp, S. (2011). Mditerranean diet and type 2 diabetes risk in the European Prospective Investigation into Cancer and Nutrition (EPIC) study. The InterAct project. Diabetes Care, 34, 1913-1918.

Janssen, I., Katzmarzyk, P. T., \& Ross, R. (2002). Body mass index, wais circumference, and health risk. Evidence in support of current Nationa Institutes of Health guidelines. Archives of Internal Medicine, 162, 2074-2079.

Kaplan, G. A., \& Keil, J. E. (1993). Socioeconomic factors and cardiovascular disease. A review of the literature. Circulation, 88, 1973-1998.

Laccetti, R., Pota, A., Stranges, S., Falconi, C., Memoli, B., Bardaro, L., et al. (2013). Evidence on the prevalence and geographic distribution of major cardiovascular risk factors in Italy. Public Health Nutr, 16, 305-315.

McLeod, E. R., Campbell, K. J., \& Hesketh, K. D. (2011). Nutrition knowledge. A mediator between socioeconomic position and diet quality in Australian firsttime mothers. Journal of the American Dietetic Association, 111, 696-704. 
Mehio Sibai, A., Nasreddine, L., Mokdad, A. H., Adra, N., Tabet, M., \& Hwalla, N. (2010). Nutrition transition and cardiovascular disease risk factors in Middle East and North Africa countries. Reviewing the evidence. Annals of Nutrition $\&$ Metabolism, 57, 193-203.

Ory, M. G., Jordan, P. J., \& Bazzarre, T. (2002). The behavior change consortium. Setting the stage for a new century of health behavior-change research. Health Setting the stage for a new century

Pala, V., Sieri, S., Palli, D., Salvini, S., Berrino, F., Bellegotti, M., et al. (2003). Diet in the Italian EPIC cohorts. Presentation of data and methodological issues. Tumor 89, 594-607.

Parmenter, K., \& Wardle, J. (1999). Development of a general nutrition knowledge questionnaire for Adults. European Journal of Clinical Nutrition, 53, 298-308.

Pisani, P., Faggiano, F., Krogh, V., Palli, D., Vineis, P., \& Berrino, F. (1997). Relative validity and reproducibility of a food frequency dietary questionnaire for use in the Italian EPIC centres. International Journal of Epidemiology, 26(Suppl. 1) S152-S160.

Powers, A. R., Struempler, B. J., Guarino, A., \& Parmer, S. M. (2005). Effects of nutrition education program on the dietary behavior and nutrition knowledge of second-grade and third-grade students. Journal of School Health, 75, 129-133.
Sahyoun, N. R., Pratt, C. A., \& Anderson, A. (2004). Evaluation of nutrition education interventions for older adults. A proposed framework. Journal of the American Dietetic Association, 104, 58-69.

Sharma, S. V., Gernand, A. D., \& Day, R. S. (2008). Nutrition knowledge predicts eating behavior of all food groups except fruits and vegetables among adults in the Paso del Norte region. Qué Sabrosa Vida. Journal of Nutrition Education and Behavior, 40, 361-368.

Sofi, F., Abbate, R., Gensini, G. F., \& Casini, A. (2010). Accruing evidence on benefits of adherence to the Mediterranean diet on health. An updated systematic review and meta-analysis. American Journal of Clinical Nutrition, 92, 1189-1196.

Tangney, C. C., Kwasny, M. J., Li, H., Wilson, R. S., Evans, D. A., \& Morris, M. C. (2011) Adherence to a Mediterranean-type dietary pattern and cognitive decline in community population. American Journal of Clinical Nutrition, 93, 601-607.

Trichopoulou, A., Bamia, C., \& Trichopoulos, D. (2009). Anatomy of health effects of Mediterranean diet. Greek EPIC prospective cohort study, BMJ, 23(338), b2337.

Trichopoulou, A., Costacou, T., Bamia, C., \& Trichopoulos, D. (2003). Adherence to Mediterranean diet and survival in a Greek population. New England Journal of Mediterranean diet and survive.
Medicine, 348, 2599-60819.

Wardle, J., Parmenter, K., \& Waller, J. (2000). Nutrition knowledge and food intake. Appetite, 34, 26-275. 



\section{CHAPTER 9.}

Decline of the Mediterranean diet at a time of economic crisis. Results from the Moli-sani study

Bonaccio M, Di Castelnuovo A, Bonanni A, Costanzo S, De Lucia F, Persichillo M, Zito F, Donati MB, de Gaetano G, Iacoviello L, on behalf of the Moli-sani project Investigators

Nutr Metab Cardiovascular Dis. 2014;24:853-60. 


\title{
Decline of the Mediterranean diet at a time of economic crisis. Results from the Moli-sani study
}

\author{
M. Bonaccio ${ }^{\mathrm{a}, *, 1}$, A. Di Castelnuovo ${ }^{\mathrm{a}, 1}$, A. Bonanni ${ }^{\mathrm{b}, 1}, \mathrm{~S}$. Costanzo ${ }^{\mathrm{a}, 1}, \mathrm{~F}$. De Lucia ${ }^{\mathrm{c}, 1}$, \\ M. Persichillo ${ }^{\mathrm{a}, 1}$, F. Zito ${ }^{\mathrm{d}, 1}$, M.B. Donati ${ }^{\mathrm{a}, 1}$, G. de Gaetano ${ }^{\mathrm{a}, 1}$, L. Iacoviello ${ }^{\mathrm{a}, 1}$ \\ ${ }^{a}$ Department of Epidemiology and Prevention, IRCCS Istituto Neurologico Mediterraneo Neuromed, Via dell'Elettronica, 86077 Pozzilli (Isernia), Italy \\ ${ }^{b}$ Epicomed Research Srl, 86100 Campobasso, Italy \\ ${ }^{c}$ Associazione Cuore Sano ONLUS, Campobasso, Italy \\ ${ }^{d}$ Transfusion Unit, Ospedale di Isernia, Isernia, Italy
}

Received 18 October 2013; received in revised form 13 February 2014; accepted 15 February 2014

Available online 1 March 2014

\section{KEYWORDS \\ Mediterranean Diet; \\ Economic crisis; \\ socioeconomic status; \\ Obesity; \\ Cerebrovascular risk}

\begin{abstract}
Background and aims: Adherence to Mediterranean diet (MD) is reportedly declining in the last decades. We aimed to investigate the adherence to MD over the period 2005-2010 and exploring the possible role of the global economic crisis in accounting for the changing in the dietary habits in Italy.

Methods and results: Cross-sectional analysis in a population-based cohort study which randomly recruited 21,001 southern Italian citizens enrolled within the Moli-sani study. Food intake was determined by the Italian EPIC food frequency questionnaire. Adherence to MD was appraised by the Italian Mediterranean Index (IMI). A wealth score was derived to evaluate the economic position and used together with other socioeconomic indicators. Highest prevalence of adherence to MD was observed during the years 2005-2006 (31.3\%) while the prevalence dramatically fell down in the years $2007-2010(18.3 \% ; P<0.0001)$. The decrease was stronger in the elderly, less affluent groups, and among those living in urban areas. Accordingly, we observed that in 2007-2010 socioeconomic indicators were strongly associated with higher adherence to MD, whereas no association was detected in the years before the economic crisis began; both wealth score and education were major determinants of high adherence to MD with 31\% (95\%CI: 18 $-46 \%$ ) higher adherence to this pattern within the wealthier group compared to the less affluent category.

Conclusion: Adherence to MD has considerably decreased over the last few years. In 2007-2010 socioeconomic indicators have become major determinants of adherence to MD, a fact likely linked to the economic downturn.

( 2014 Elsevier B.V. All rights reserved.
\end{abstract}

\section{Introduction}

Cardiovascular disease (CVD), cancer, obesity and type 2 diabetes account for $70 \%$ of all deaths in Europe every year [1]. These non-communicable diseases have been shown

\footnotetext{
* Corresponding author. Tel.: +39 (0)865929665; fax: +39 (0) 865927575.

E-mail addresses: marialaura.bonaccio@neuromed.it (M. Bonaccio), licia.iacoviello@moli-sani.org (L. Iacoviello).

${ }^{1}$ On behalf of the Moli-sani project Investigators listed in Appendix 1.
}

to be largely preventable by promoting healthy lifestyles, such as healthy diets, physical activity, tobacco cessation and moderate alcohol consumption. Adherence to a Mediterranean dietary pattern has been widely associated with reduced risk for major chronic diseases, including neurodegenerative diseases, and reduced mortality for CVD [2-4].

Studies conducted within Mediterranean populations have shown that adherence to the Mediterranean diet (MD) has gradually declined in the last decades [5-7]. The social and cultural changes occurred during the last years 
and globalization have deeply influenced the lifestyle of people yielding a gradual shifting towards more Westerntype ways of living and eating habits and the more affected countries resulted to be the ones in the Mediterranean basin $[8,9]$.

Recent evidence shows that more disadvantaged people are less likely to follow the traditional MD compared to those with higher household income, independently from other socioeconomic indicators [10]. The cost of healthy foods has been suggested to play a role in the dietary choices of people [11] whilst the low cost and high palatability of energy-dense foods is rising concern among the scientific community [12].

The occurrence of the global economic crisis started in 2007 is likely to have worsened the situation mainly for disadvantaged groups. In addition, there is major concern about possible unfavourable outcomes of the global economic crisis on health mainly due to the fiscal austerity adopted by European governments. Recent investigations have highlighted how policies inspired to restriction have deeply affected the health status of people, mainly in the weakest European Community Countries, such as Greece and Italy [13,14].

The aim of this study was to investigate the trend in adherence to MD over the very last years and to explore the role of the global economic crisis and the accounting of socioeconomic indicators for the adherence to the MD before and after the economic downturn.

\section{Methods}

\section{Study population}

Between March 2005 and April 2010, 24,325 participants (men and women aged $\geq 35$ years) were randomly recruited within a population-based cohort study (Molisani), in the Italian region of Molise, an area placed between Central and Southern Italy ([10] and Appendix 2).

After exclusion of subjects reporting personal history of cardiovascular disease (angina, myocardial infarction, heart failure, revascularization procedures and stroke; $5.7 \%)$, cancer $(3.1 \%)$ or of those for whom there were no available information on dietary habits (3.9\%), 21,001 subjects were finally included in the analysis.

\section{Dietary information}

The validated Italian EPIC food frequency questionnaire was used to evaluate food intake $[15,16]$. To simplify interpretation of data and to minimize within-person variations in intakes of individual foods, 188 food items were classified into 45 predefined food groups on the basis of similar nutrient characteristics or culinary usage (Appendix 3).

Moderate alcohol intake was defined as regularly drinking less than two or one drinks a day, by men or women, respectively.

We evaluated adherence to Mediterranean diet by using the Italian Mediterranean Index (IMI) a score conceived to better capture healthy eating including foods, such as pasta, more typically consumed in Italy $[10,17]$.

Total food antioxidant content (FAC) score was used to assess the antioxidant content from diet [18].

\section{Socioeconomic indicators}

Household income was divided into four categories as low ( $\leq 10,000$ Euros/year; 0 point), low-medium $(>10,000$ $\leq 25,000$ Euros/year; 1 point), medium-high $(>25,000$ $\leq 40,000$ Euros/year; 2 points) and high ( $>40,000$ Euros/ year; 3 points). A specific variable for missing values for income (32.2\%) was created for not excluding from the analysis such a large representative number of people for whom other socioeconomic information were still available. This category was labelled as "non-respondent". Housing was considered as rented ( 0 point), one dwelling ownership ( 2 points) and more than one dwelling ownership (3 points).

The household wealth score was obtained by the ratio between household income plus housing and then divided by the number of living-in members and ranged between 0 and 6 . The score was then categorized as low $(<1)$, medium $(\geq 1$ and $\leq 1.25)$ and high $(>1.25)$ which represent approximate tertiles of the population for whom the score was not missing. Missing values (32.4\%) were labelled as "non-respondent".

Education was divided into three levels: $\leq 8$ (years of studies), 8-13 and $>13$. Marital status was intended as married/living-in partners, divorced, single or widow. Profession was considered as manual, not manual or other (retired, housewife, etc.). This study also considered the site of residence and it was dichotomized as resident in a city (number of inhabitants $>8000$ ) or in smaller villages.

Annual average rate of price change of some foods as measured by the Harmonised Index of Consumer Prices (HICP) was used for a comparison with the trend of adherence to MD in our population.

\section{Statistics}

Values for continuous variables are presented as mean$\mathrm{s} \pm$ Standard Deviation. Analysis of variance for continuous or categorical variables was applied to test the associations in Tables 1, 2 and 4. High adherence to MD was defined when the Italian Mediterranean Index was $\geq 5$ points.

Multivariable binomial (Poisson) regression with the log link function [19] was used to quantify the associations between high $(\geq 5)$ vs. low $(<5)$ adherence to the MD with anthropometric or socioeconomic variables. Potential confounders included as covariates in the model were total energy intake, total physical activity, BMI, smoking, hypertension, hypercholesterolaemia and diabetes. An appropriate interaction term between recruitment period (2005-2006 and 2007-2010) and each of the presented variables was introduced to test whether the changes in the adherence to MD in the two enrolment periods were different according to the levels of each variable. 
Table 1 Characteristics of the population sample overall and by period of recruitment

\begin{tabular}{|c|c|c|c|c|c|}
\hline & \multirow{2}{*}{$\frac{\text { Overall }}{2005-2010 n=21001}$} & \multicolumn{2}{|l|}{ By recruitment period } & \multirow[t]{2}{*}{ \% Difference } & \multirow[t]{2}{*}{$P$ value $^{a}$} \\
\hline & & $2005-2006 n=6999$ & $2007-2010 n=14002$ & & \\
\hline Age (means $\pm \mathrm{SD}$ ) & $54.4(11.4)$ & $54.6(11.9)$ & $54.3(11.1)$ & -0.8 & 0.03 \\
\hline Sex (Men, $n$ and \%) & $9932(47.3)$ & $3283(46.9)$ & $6649(47.5)$ & +0.6 & 0.40 \\
\hline \multicolumn{6}{|l|}{ Physical activity ( $n, \%)$} \\
\hline Low & $6474(30.8)$ & $2126(30.4)$ & $4348(31.5)$ & +1.1 & $<0.0001$ \\
\hline Moderate & $7641(36.4)$ & $2591(37.0)$ & $5050(36.1)$ & -0.9 & \\
\hline High & 6708 (31.9) & $2258(32.3)$ & $4450(31.8)$ & -0.5 & \\
\hline \multicolumn{6}{|l|}{ Smoking $(n, \%)$} \\
\hline Never & $10511(50.0)$ & 3452 (49.3) & $7059(50.4)$ & +1.1 & 0.06 \\
\hline Current & $5042(24.0)$ & $1740(24.9)$ & $3302(23.6)$ & -1.3 & \\
\hline Former & $5448(26.0)$ & $1807(25.8)$ & $3641(26.0)$ & +0.2 & \\
\hline \multicolumn{6}{|l|}{ Wealth score $(n, \%)$} \\
\hline Low & $4581(21.8)$ & $1429(20.4)$ & $3152(22.5)$ & +2.1 & $<0.0001$ \\
\hline Medium & $4606(21.9)$ & $1685(24.1)$ & $2921(20.9)$ & -3.2 & \\
\hline High & $5000(23.8)$ & $2082(29.7)$ & $2918(20.8)$ & -8.9 & \\
\hline Non-respondent & $6814(32.4)$ & $1803(25.8)$ & $5001(35.8)$ & +10.0 & \\
\hline \multicolumn{6}{|l|}{ Education ( $n, \%$; years) } \\
\hline$\leq 8$ & $10755(50.4)$ & $3216(46.0)$ & $7539(53.8)$ & +7.8 & $<0.0001$ \\
\hline$>8 \leq 13$ & $7482(36.2)$ & $2666(39.1)$ & 4627 (33.9) & -5.2 & \\
\hline$>13$ & $2751(13.3)$ & $1021(14.9)$ & $1669(12.2)$ & -2.7 & \\
\hline \multicolumn{6}{|l|}{ Site of residence $(n, \%)$} \\
\hline Village & $7562(36.0)$ & $1453(20.8)$ & $6109(43.6)$ & +22.8 & $<0.0001$ \\
\hline City & $13439(64.0)$ & $5546(79.2)$ & $7893(56.4)$ & -22.8 & \\
\hline Obesity $(n, \%)$ & $6049(28.8)$ & $1870(26.7)$ & 4179 (29.9) & +3.2 & $<0.0001$ \\
\hline Hypertension ( $n, \%)$ & $11298(53.8)$ & $4035(57.6)$ & $7263(51.9)$ & -5.7 & $<0.0001$ \\
\hline Diabetes $(n, \%)$ & $1683(8.0)$ & $498(7.1)$ & $1185(8.5)$ & +1.4 & $<0.0001$ \\
\hline Hypercholesterolaemia ( $n, \%)$ & $6142(29.2)$ & $1718(24.5)$ & $4424(31.6)$ & +7.1 & $<0.0001$ \\
\hline
\end{tabular}

The data analysis was generated using SAS/STAT software, Version 9.1.3 of the SAS System for Windows $\odot 2009$. SAS Institute Inc. and SAS are registered trademarks of SAS Institute Inc., Cary, NC, USA.

\section{Results}

When the recruitment period was divided by each year of enrolment, a clear drop in adherence to MD was recorded starting from 2007 (Fig. 1). Prevalence of high adherence (IMI $\geq 5$ ) fell from $33.7 \%$ in 2005 to $19.6 \%$ in 2007 and this negative trend remained in the following years with the lowest rate recorded in 2009 (14.7\%; Fig. 1).

For the subsequent analysis we finally considered two main periods of time (2005-2006 and 2007-2010). Characteristics of the overall population sample and of these two sub-samples are shown in Table 1. Major differences between the two sub-groups are related to socioeconomic indicators $(P<0.0001)$.

Prevalence of high adherence to MD over time and according to socioeconomic and demographic indicators is reported in Table 2. In the whole period of recruitment, adherence to MD increased following an age gradient up to the 60-70 years group whilst it decreased in the eldest category ( $>70$ years). Women, or more affluent and educated people, or those having a not manual job, or single persons or those living in urban areas reported the highest adherence to MD (Table 2).
In 2007-2010 high adherence to MD decreased by 13 points as compared to the previous period $(P<0.0001$; Table 2). The strongest decrease was observed in adult people compared to youngest groups $(P$ for interaction $=0.026$; Table 2 ), less affluent groups ( $P$ for interaction $=0.032$; Table 2 ), less educated people ( $P$ for interaction $=0.016$; Table 2 ), manual workers ( $P$ for interaction $=0.022$; Table 2 ) and people living in urban areas ( $P$ for interaction $<0.0001$; Table 2 ).

Poisson regression analysis was used to determine the accounting of major socioeconomic indicators for the differences in adherence to MD over time (Table 3). In 2005-2006 the association between high adherence to MD and main socioeconomic determinants was not significant, except for few indicators such as education, with more educated people ( $\geq 13$ years of study) reporting $16 \%$ higher adherence to MD compared to those with lower education, and people living in urban areas (Table 3 ).

In 2007-2010 socioeconomic factors accrued their contribution with regard to higher adherence to MD. Subjects with the highest household wealth score had 31\% higher adherence to MD (prevalence ratio $=1.31 ; 95 \% \mathrm{Cl}$ 1.18-1.46). Education also reinforced its influence with highest educated individuals being 1.32 times as likely to have higher adherence to MD (prevalence ratio $=1.32$; 95\%CI 1.17-1.50) as the least educated group ( $\leq 8$ years of study). Regarding profession, in 2007-2010 manual workers reported poorer adherence to $\mathrm{MD}$ as well as 
Table 2 Prevalence of high adherence to the Mediterranean diet (Italian Mediterranean index $\geq 5$ points) according to socioeconomic indicators in the whole and by recruitment periods.

\begin{tabular}{|c|c|c|c|c|c|}
\hline \multirow[t]{2}{*}{$\mathrm{IMI} \geq 5$ points } & \multirow{2}{*}{$\frac{\text { Overall }}{2005-2010}$} & \multicolumn{2}{|c|}{ By recruitment period } & \multirow[t]{2}{*}{ Difference $^{a}$} & \multirow[t]{2}{*}{$P$ value $^{b}$} \\
\hline & & 2005-2006 & 2007-2010 & & \\
\hline Whole sample & $4755(22.6)$ & 2191(31.3) & $2564(18.3)$ & -13.0 & $<0.0001$ \\
\hline \multicolumn{6}{|l|}{ Age groups $(n, \%)$} \\
\hline $35-43$ & $807(20.8)$ & $367(26.3)$ & 440 (17.7) & -8.6 & $<0.0001$ \\
\hline $44-53$ & $1428(21.7)$ & $640(31.3)$ & $788(17.4)$ & -13.9 & $<0.0001$ \\
\hline $54-59$ & $923(25.3)$ & 403 (33.9) & $520(21.1)$ & -12.8 & $<0.0001$ \\
\hline $60-70$ & $1147(25.1)$ & $541(35.8)$ & $606(19.8)$ & -16.0 & $<0.0001$ \\
\hline \multirow[t]{2}{*}{$>70$} & $450(19.4)$ & $240(27.8)$ & $210(14.4)$ & -13.4 & $<0.0001$ \\
\hline & $P<0.0001^{\mathrm{C}}$ & $P<0.0001$ & $P<0.0001$ & $P$ for interaction $=0.026$ & \\
\hline \multicolumn{6}{|l|}{$\operatorname{Sex}(n, \%)$} \\
\hline Women & $2567(23.2)$ & $1203(32.4)$ & $1364(18.5)$ & -13.9 & $<0.0001$ \\
\hline \multirow[t]{2}{*}{ Men } & $2188(22.0)$ & $988(30.1)$ & $1200(18.0)$ & -12.1 & $<0.0001$ \\
\hline & $P=0.04$ & $P=0.04$ & $P=0.45$ & $P$ for interaction $=0.29$ & \\
\hline \multicolumn{6}{|l|}{ Wealth score $(n, \%)$} \\
\hline Low & $970(21.2)$ & $432(30.2)$ & $538(17.1)$ & -13.1 & $<0.0001$ \\
\hline Medium & $1083(23.5)$ & 535 (31.7) & $548(18.8)$ & -12.9 & $<0.0001$ \\
\hline High & $1347(26.9)$ & $687(33.0)$ & $660(22.6)$ & -10.4 & $<0.0001$ \\
\hline \multirow[t]{2}{*}{ Non-respondent } & 1355 (19.9) & $537(29.8)$ & $818(16.3)$ & -13.5 & $<0.0001$ \\
\hline & $P<0.0001$ & $P=0.09$ & $P<0.0001$ & $P$ for interaction $=0.032$ & \\
\hline \multicolumn{6}{|l|}{ Education ( $n, \%$; years) } \\
\hline$\leq 8$ & $2234(20.8)$ & 989 (30.7) & $1245(16.5)$ & -14.2 & $<0.0001$ \\
\hline$>8 \leq 13$ & $1792(23.9)$ & 839 (30.7) & $953(20.1)$ & -10.6 & $<0.0001$ \\
\hline \multirow[t]{2}{*}{$>13$} & $728(26.5)$ & $363(34.7)$ & $365(21.4)$ & -13.3 & $<0.0001$ \\
\hline & $P<0.0001$ & $P=0.014$ & $P<0.0001$ & $P$ for interaction $=0.016$ & \\
\hline \multicolumn{6}{|l|}{ Profession $(n, \%)$} \\
\hline Not manual & $1232(24.5)$ & 609 (31.4) & $623(20.1)$ & -11.3 & $<0.0001$ \\
\hline Manual & $460(19.2)$ & $188(31.4)$ & $272(15.1)$ & -16.3 & $<0.0001$ \\
\hline \multirow[t]{2}{*}{ Other (retired, housewife, etc.) } & $3059(22.6)$ & $1392(31.2)$ & $1667(18.3)$ & -12.9 & $<0.0001$ \\
\hline & $P<0.0001$ & $P=0.46$ & $P<0.0001$ & $P$ for interaction $=0.022$ & \\
\hline \multicolumn{6}{|l|}{ Marital status $(n, \%)$} \\
\hline Married or living-in partners & $4094(22.7)$ & $1875(31.5)$ & $2219(18.3)$ & -13.2 & $<0.0001$ \\
\hline Divorced & $136(24.3)$ & $61(29.8)$ & $75(21.2)$ & -8.6 & 0.020 \\
\hline Single & $268(24.0)$ & $120(31.1)$ & $148(20.2)$ & -10.9 & $<0.0001$ \\
\hline \multirow[t]{2}{*}{ Widow } & $257(20.2)$ & $135(29.7)$ & $122(15.0)$ & -14.7 & $<0.0001$ \\
\hline & $P=0.01$ & $P=0.26$ & $P=0.016$ & $P$ for interaction $=0.29$ & \\
\hline \multicolumn{6}{|l|}{ Site of residence $(n, \%)$} \\
\hline Village & $1509(20.0)$ & $363(25.0)$ & $1146(18.8)$ & -6.2 & $<0.0001$ \\
\hline \multirow[t]{2}{*}{ City } & $3246(24.1)$ & $1828(33.0)$ & $1418(18.0)$ & -15.0 & $<0.0001$ \\
\hline & $P<0.0001$ & $P<0.0001$ & $P=0.23$ & $P$ for interaction $<0.0001$ & \\
\hline
\end{tabular}

All $P$ values reported in this table are from analysis of variance for categorical data where the dependent variable was having IMI $\geq 5$, adjusted for age and sex.

Difference in the percentage of subjects having IMI $\geq 5$ between the periods 2007-2010 and 2005-2006.

${ }^{b} P$ value for difference between the two periods of time. $P$ values are reported for the whole sample and for each strata of the indicated variables.

${ }^{c} P$ value for the difference within levels of each indicated variable, in the whole sample and within each time of period. $P$ for interaction tests whether the changes in the adherence to MD in the two enrolment periods were different according to the levels of each indicated variable.

urban residents (Table 3). Despite being still significant, the relationship between age groups and adherence to MD appeared to be reduced after the crisis began. In 2005-2006 prevalence ratio for adult subjects (60-70 years) was 1.50 , in comparison with the youngest age group (35-43 years), whereas in 2007-2010 the prevalence ratio was reduced to 1.27 . The same drastic negative trends were recorded for all age groups, also for the eldest one in whom, in 2007-2010 period, the relation was even not significant (Table 3). Additional analyses performed by using the Mediterranean diet score [20] or by excluding diabetes subjects provided similar results (data not shown). When comparing data from 2005 (highest adherence to MD) with those in 2009 (lowest adherence) we found the main socioeconomic variables being associated with higher adherence to MD in 2009 and not in 2005 , although the association was not statistically significant likely due to the small number of subjects having 


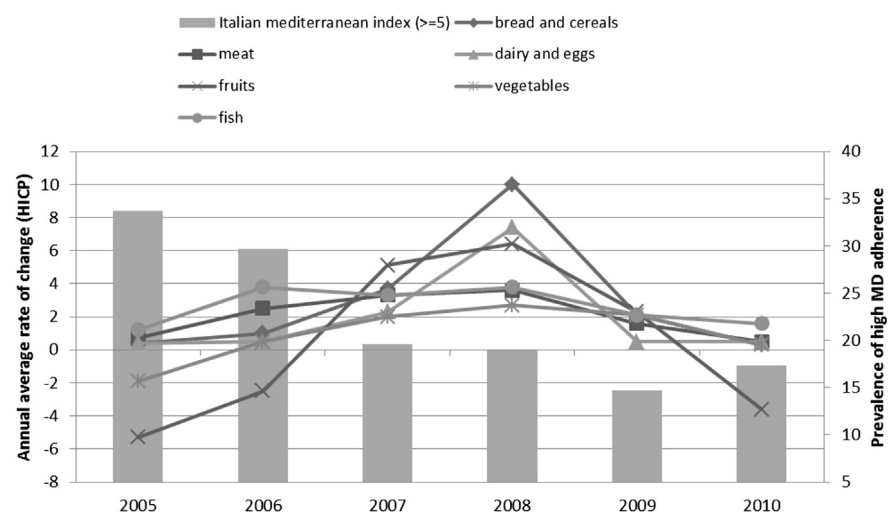

Figure 1 Bars (vertical axis on the right) represent the prevalence of high adherence to Mediterranean diet (IMI $\geq 5$ ) over time as observed in our sample. Each line (vertical axis on the left) represents the annual average rate of change of the Harmonised Index of Consumer Prices of selected foods typical of the Mediterranean dietary pattern (source: ISTAT). The dramatic drop in the adherence to the Mediterranean diet was firstly recorded in 2007 when the cost of some key foods started to rise.

high adherence to MD in $2009(n=422)$ (data not shown).

Table 4 shows the differences in nutrients intake over time. In 2007-2010 there was an increase in the intake of animal proteins, animal or saturated fat $(P<0.0001)$ and alcohol $(P=0.0013)$; conversely, we recorded a significant decrease in the intake of vegetarian fats and proteins, folic acid, dietary antioxidant content, fibres and carbohydrates $(P<0.0001)$.

Regarding the contribution of macronutrients to the daily energy intake in 2007-2010, proteins and carbohydrates decreased over time $(P$ value $<0.0001)$, while fats increased $(P$ value $<0.0001)$. The contribution of alcohol remained stable over time $(P$ value $=0.39)$.

Fig. 1 shows that in 2007-2008 the change of HICP reached its peak for many typical Mediterranean food items such as fruits, vegetables, bread and cereals. In the same time, we recorded a dramatic overall shift from MD.

\section{Discussion}

Health outcomes deriving from the present economic crisis can be just predicted on the basis of what happened during the former economic downturns, even though some preliminary estimations have already been addressed $[13,14]$.

This study represents one of the first attempts to provide a consistent evaluation of the accounting of the global economic crisis for the change in dietary habits of a large, representative sample of Italian population. The main outcome considered in this analysis is the adherence to MD, a healthy eating pattern largely linked to a reduction of major risk factors for non-communicable diseases and to a significant decrease in terms of mortality rates [2,3].

Our study has highlighted that the drifting away from the traditional MD interested all age and socioeconomic groups. In respect to the years 2005-2006, a dramatic drop was recorded in 2007, the period in which the global economic crisis became manifested, with prevalence of high adherence falling from over $30 \%$ to $18 \%$ in the whole population.

Being in the adult group (60-70 years) in 2005-2006 meant having a greater prevalence of high adherence to the MD, but in 2007-2010 the same age group, as well as those in the 44-45 age group, experienced a strong reduction in terms of prevalence of high adherence to this dietary pattern. Following the traditional MD has largely been considered as a matter of culture typical of oldest groups with different eating behaviours compared to the youngest generations [5]. The differences observed between the two periods of time lead us to speculate that during the very last years, being part of an older generation did not necessarily imply maintaining the traditional dietary habits, even though the gap between adult and youngest individuals was still present. The negative trend in adherence to MD could be detected for each socioeconomic parameter too. Major decreases were experienced by low-medium household wealth groups, low and highly educated individuals, old people, manual workers and for subjects living in urban areas.

Our hypothesis that the global economic crisis could play a role in the observed sudden decline of MD adherence was investigated by the contribution of different socioeconomic indicators to the adherence to the Mediterranean dietary pattern over the two periods of time. During 2005-2006 major socioeconomic indicators were not associated with higher adherence to MD, which was conversely linked to age, sex, higher education and living in urban areas. In particular, differences among wealth groups in adherence to MD were not significant.

In 2007-2010, in contrast, socioeconomic determinants began to play a major role in explaining the positive association with MD. Greater household wealth, higher education, living in small villages and type of job became 
Table 3 Prevalence ratio (PR) of high adherence to the Mediterranean diet (Italian Mediterranean index $\geq 5$ points) according to socioeconomic indicators over time.

\begin{tabular}{|c|c|c|}
\hline & \multicolumn{2}{|c|}{ Italian Mediterranean index $(\geq 5)$} \\
\hline & \multirow{2}{*}{$\begin{array}{l}2005-2006 \\
(n=6999) \\
\operatorname{PR}(95 \% \mathrm{CI})\end{array}$} & \multirow{2}{*}{$\begin{array}{l}2007-2010 \\
(n=14,002) \\
\operatorname{PR}(95 \% \mathrm{CI})\end{array}$} \\
\hline & & \\
\hline \multicolumn{3}{|l|}{ Age groups } \\
\hline $35-43$ & $-1-$ & $-1-$ \\
\hline $44-53$ & $1.21(1.09-1.35)$ & $1.01(0.91-1.12)$ \\
\hline $54-59$ & $1.35(1.19-1.53)$ & $1.30(1.15-1.47)$ \\
\hline $60-70$ & $1.50(1.32-1.70)$ & $1.27(1.12-1.45)$ \\
\hline$>70$ & $1.35(1.14-1.59)$ & $1.12(0.94-1.34)$ \\
\hline \multicolumn{3}{|l|}{ Sex } \\
\hline Women & $-1-$ & $-1-$ \\
\hline Men & $0.77(0.71-0.83)$ & $0.75(0.69-0.82)$ \\
\hline \multicolumn{3}{|l|}{ Wealth score } \\
\hline Low & $-1-$ & $-1-$ \\
\hline Medium & $1.04(0.94-1.16)$ & $1.10(0.98-1.22)$ \\
\hline High & $1.05(0.94-1.16)$ & $1.31(1.18-1.46)$ \\
\hline Non-respondent & $1.03(0.92-1.15)$ & $1.00(0.91-1.11)$ \\
\hline \multicolumn{3}{|l|}{ Education (years) } \\
\hline$\leq 8$ & $-1-$ & $-1-$ \\
\hline$>8 \leq 13$ & $1.02(0.93-1.11)$ & $1.24(1.14-1.35)$ \\
\hline$>13$ & $1.16(1.04-1.31)$ & $1.32(1.17-1.50)$ \\
\hline \multicolumn{3}{|l|}{ Profession } \\
\hline Not manual & $-1-$ & $-1-$ \\
\hline Manual & $0.97(0.82-1.15)$ & $0.67(0.57-0.79)$ \\
\hline $\begin{array}{l}\text { Other (retired, } \\
\text { housewife, etc.) }\end{array}$ & $0.99(0.90-1.10)$ & $0.92(0.83-1.03)$ \\
\hline \multicolumn{3}{|l|}{ Marital status } \\
\hline $\begin{array}{l}\text { Married } \\
\text { or living-in partners }\end{array}$ & $-1-$ & $-1-$ \\
\hline Divorced & $0.95(0.77-1.18)$ & $1.10(0.90-1.33)$ \\
\hline Single & $1.07(0.92-1.24)$ & $1.13(0.97-1.32)$ \\
\hline Widow & $0.96(0.82-1.12)$ & $0.90(0.76-1.08)$ \\
\hline \multicolumn{3}{|l|}{ Site of residence } \\
\hline Village & $-1-$ & $-1-$ \\
\hline City & $1.30(1.18-1.44)$ & $0.93(0.86-0.99)$ \\
\hline
\end{tabular}

largely associated with higher adherence to MD. The reduction in pension values in Italy may account for the poorer adherence to MD recorded in the eldest group ( $>70$ years) as well as in the retired group.

The huge decline of MD detected in urban areas may be better understood if we consider that individuals living in small villages usually grow their own fruit and vegetables, including olive oil, and this fact can generate considerable savings for the household food expenditure.

The growing importance of socioeconomic indicators in determining dietary habits may find a likely explanation either in the rising cost of typical Mediterranean food items or in the higher rates of unemployment and reduction in terms of purchasing power of wages.

As shown in Fig. 1, the annual average rate of change of the HICP indicates that the cost of some basic products has risen consistently over the very last years, in particular during the two-year period 2007-2008 as the economic downturn started to take effect.

A large body of evidence demonstrated that diet quality follows a socioeconomic gradient in every time and independently from economic downturns, with more affluent groups having healthier dietary habits, but there is reason to believe that recession first, and consequent austerity policies then, could increase disparities among groups. Saving money by cutting on healthy food is going to have negative consequences for health and the health system. Although a gradual shift from the MD was firstly observed several decades ago the most dramatic drop was recorded in the very last years. During the period of 1991-1994 the average adherence to MD by a sample of the Italian population was 4.62, as measured by the Greek Mediterranean score [20]. In 2000-2006, the mean adherence to this pattern was 4.59 , less than $1 \%$ decrease [21]. Data from our population showed that average adherence to MD declined from 4.78 in 2005-2006 to 4.13 in 2009-2010, a decrease of $13.6 \%$ in very few years (data not shown). This unexpected drop recorded in a very short time span led us to suppose that the average negative trend was somehow accelerated by new factors and the economic crisis could be likely identified as one of the most influent in determining the shift towards alternative ways of eating.

To our knowledge, this is the first study addressing a possible link between the current global economic crisis and the dramatic drop in adherence to MD occurred in a large representative sample of Italian population during the very last years. The present study was cross-sectional

Table 4 Differences in micronutrient and macronutrient intake over time

\begin{tabular}{|c|c|c|c|}
\hline \multirow[t]{2}{*}{ Grams/day (means \pm SD) } & \multicolumn{2}{|c|}{ Period of recruitment } & \multirow[t]{2}{*}{$P$ value ${ }^{a}$} \\
\hline & $2005-2006$ & $2007-2010$ & \\
\hline \multicolumn{4}{|l|}{ Decreased intake } \\
\hline Vegetarian proteins & $29.6(11.1)$ & 28.7 (9.9) & $<0.0001$ \\
\hline Vegetarian fats & $33.1(11.8)$ & $32.5(10.6)$ & $<0.0001$ \\
\hline Fibres & $21.8(7.6)$ & $20.0(6.5)$ & $<0.0001$ \\
\hline Carbohydrates & $261.8(95.1)$ & $258.5(86.5)$ & $<0.0001$ \\
\hline Folic acid (mcg/day) & $266.1(88.2)$ & $259.6(75.5)$ & $<0.0001$ \\
\hline $\begin{array}{c}\text { Dietary antioxidant } \\
\text { content (score) }\end{array}$ & $5.9(50.2)$ & $-3.4(47.6)$ & $<0.0001$ \\
\hline \multicolumn{4}{|l|}{ Increased intake } \\
\hline Animal & $55.1(18.7)$ & $56.4(17.0)$ & $<0.0001$ \\
\hline Animal fats & $44.2(18.8)$ & $45.3(17.0)$ & $<0.0001$ \\
\hline ed fats & $27.7(11.0)$ & $28.1(9.8)$ & $<0.0001$ \\
\hline Alcohol & $16.0(21.8)$ & $16.9(23.2)$ & 0.0013 \\
\hline \multicolumn{4}{|c|}{ Macronutrients contribution to energy intake (\%) } \\
\hline $\begin{array}{l}\text { Energy intake } \\
\quad(\text { means } \pm \mathrm{SD})\end{array}$ & $2228(675)$ & $2101(614)$ & $<0.0001$ \\
\hline Proteins (\%) & 15.5 & 15.8 & $<0.0001$ \\
\hline Carbohydrates (\%) & 47.6 & 46.9 & $<0.0001$ \\
\hline Fats (\%) & 31.8 & 32.3 & $<0.0001$ \\
\hline Alcohol (\%) & 5.0 & 4.9 & 0.39 \\
\hline
\end{tabular}

a Means and $P$ value adjusted for sex, age and energy intake. 
and shares all the limitations of this study type. Dietary and socioeconomic information were self-reported, although questionnaires were administered by trained researchers.

Caution is needed in extending the results presented here to larger contexts since data were collected in a region located between Central and Southern Italy, Mediterranean by tradition and culture [10]. Yet, the main characteristics of our population sample are comparable to those of the Italian Cardiovascular Epidemiological Observatory [22], therefore our sample could be considered representative at least of the Italian population.

In conclusion, socioeconomic factors have become major determinants of adherence to MD and their growing weight is likely to increase health inequalities with individuals from lower classes having reduced chances to follow healthy diets, thus increasing their risk for major chronic diseases.

\section{Sources of funding}

The enrolment phase of the Moli-sani Project was supported by research grants from Pfizer Foundation (Rome, Italy) and the Italian Ministry of University and Research (MIUR, Rome, Italy)-Programma Triennale di Ricerca, Decreto No. 0.1588. Pfizer Foundation and MIUR had no role in study design, collection, analysis, and interpretation of data; in the writing of the report; and in the decision to submit the article for publication. All Authors were and are independent from funders.

\section{Competing interest statement}

None of the Authors had a personal or financial conflict of interest.

\section{Authors' contributions to manuscript}

$\mathrm{MB}, \mathrm{ADC}$, LI designed the research; FDL, MB, MP, SC, FZ managed data collection; $\mathrm{MB}, \mathrm{ADC}$ analysed the data; $\mathrm{MB}, \mathrm{AB}$ wrote the paper; MBD, GdG, LI originally inspired the research, obtained the financial support and critically reviewed the manuscript. All Authors had full access to all of the data in the study and take responsibility for the integrity of the data and the accuracy of the data analysis.

\section{Acknowledgements}

The enrolment phase of the Moli-sani Project was performed at the Research Laboratories, Catholic University, Campobasso, Italy. The Moli-sani research group would like to thank Drs. Vittorio Krogh and Sabina Sieri from Istituto Nazionale dei Tumori, Milan, Italy, for their contribution to dietary questionnaire analysis and interpretation.

\section{Appendix A. Supplementary data}

Supplementary data related to this article can be found at http://dx.doi.org/10.1016/j.numecd.2014.02.014.

\section{References}

[1] World Health Organization. 2008-2013 Action plan for the global strategy for the prevention and control of non communicable diseases. Geneva: World Health Organization; 2008.

[2] Fitó M, Estruch R, Salas-Salvadó J, Martínez-Gonzalez MA, Arós F Vila J, et al. Primary prevention of cardiovascular disease with a Mediterranean diet. N Engl J Med 2013;368:1279-90.

[3] Sofi F, Abbate R, Gensini GF, Casini A. Accruing evidence on benefits of adherence to the Mediterranean diet on health: an updated systematic review and meta-analysis. Am J Clin Nutr 2010;92: 1189-96.

[4] Bonaccio M, Iacoviello L, de Gaetano G, On Behalf of the Moli-Sani Investigators. The Mediterranean diet: the reasons for a success. Thromb Res 2012;129:401-4.

[5] di Giuseppe R, Bonanni A, Olivieri M, Di Castelnuovo A, Donati MB, de Gaetano G, et al. Adherence to Mediterranean diet and anthropometric and metabolic parameters in an observational study in the 'Alto Molise' region: the MOLI-SAL project. Nutr Metab Cardiovasc Dis 2008;18:415-21.

[6] León-Muñoz LM, Guallar-Castillón P, Graciani A, López-García E, Mesas AE, Aguilera MT, et al. Adherence to the Mediterranean diet pattern has declined in Spanish adults. J Nutr 2012;142:1843-50.

[7] Sofi F, Vecchio S, Giuliani G, Martinelli F, Marcucci R, Gori AM, et al. Dietary habits, lifestyle and cardiovascular risk factors in a clinically healthy Italian population: the 'Florence' diet is not Mediterranean. Eur J Clin Nutr 2005;59:584-91.

[8] Serra-Majem L, Trichopoulou A, Ngo de la Cruz J, Cervera P, García Alvarez A, La Vecchia C, et al. Does the definition of the Mediterranean diet need to be updated? Public Health Nutr 2004;7: 927-9.

[9] da Silva R, Bach-Faig A, Raidó Quintana B, Buckland G, Vaz de Almeida MD, Serra-Majem L. Worldwide variation of adherence to the Mediterranean diet, in 1961-1965 and 2000-2003. Public Health Nutr 2009;12:1676-84.

[10] Bonaccio M, Bonanni AE, Di Castelnuovo A, De Lucia F, Donati MB, de Gaetano G, et al. Low income is associated with poor adherence to a Mediterranean diet and a higher prevalence of obesity: crosssectional results from the Moli-sani study. BMJ Open 2012;2(6).

[11] Lopez CN, Martinez-Gonzalez MA, Sanchez-Villegas A, Alonso A, Pimenta AM, Bes-Rastrollo M. Costs of Mediterranean and western dietary patterns in a Spanish cohort and their relationship with prospective weight change. J Epidemiol Community Health 2009; 63:920-7.

[12] Drewnowski A. Obesity, diets, and social inequalities. Nutr Rev 2009;67(Suppl. 1):S36-9.

[13] Karanikolos M, Mladovsky P, Cylus J, Thomson S, Basu S, Stuckler D, et al. Financial crisis, austerity, and health in Europe. Lancet 2013:381:1323-31.

[14] Kondilis E, Giannakopoulos S, Gavana M, Ierodiakonou I, Waitzkin H, Benos A. Economic crisis, restrictive policies, and the population's health and health care: the Greek case. Am J Public Health 2013;103:973-9.

[15] Pala V, Sieri S, Palli D, Salvini S, Berrino F, Bellegotti M, et al. Diet in the Italian EPIC cohorts: presentation of data and methodological issues. Tumori 2003;89:594-607.

[16] Pisani P, Faggiano F, Krogh V, Palli D, Vineis P, Berrino F. Relative validity and reproducibility of a food frequency dietary questionnaire for use in the Italian EPIC centres. Int J Epidemiol 1997; 26(Suppl. 1):S152-60.

[17] Agnoli C, Krogh V, Grioni S, Sieri S, Palli D, Masala G, et al. A prioridefined dietary patterns are associated with reduced risk of stroke in a large Italian cohort. J Nutr 2011;141:1552-8.

[18] Pounis G, Costanzo S, di Giuseppe R, de Lucia F, Santimone I, Sciarretta A, et al. Consumption of healthy foods at different content of antioxidant vitamins and phytochemicals and metabolic risk factors for cardiovascular disease in men and women of the Moli-sani study. Eur J Clin Nutr 2013;67:207-13. 
[19] Spiegelman D, Hertzmark E. Easy SAS calculations for risk or prevalence ratios and differences. Am J Epidemiol 2005;162: 199-200.

[20] Trichopoulou A, Costacou T, Bamia C, Trichopoulos D. Adherence to a Mediterranean diet and survival in a Greek population. N Engl J Med 2003;348:2599-60819.
[21] Pelucchi C, Galeone C, Negri E, La Vecchia C. Trends in adherence to the Mediterranean diet in an Italian population between 1991 and 2006. Eur J Clin Nutr 2010;64:1052-6.

[22] Giampaoli S, Rielli R, Demattè L, Donfrancesco C, Lo Noce C, Dima $\mathrm{F}$, et al. The Italian observatory of cardiovascular risk: the CUORE project experience. Circulation 2009;119:139. 



\section{CHAPTER 10.}

\section{Correspondence}

Cardiovascular risk and events and country income stratum

Bonaccio M, Di Castelnuovo A, Iacoviello L

N Engl J Med. 2015;372:288-9. 
Jaime Sanz, M.D., Ph.D.

Guillermo F. Sanz, M.D., Ph.D.

Miguel A. Sanz, M.D., Ph.D.

Hospital Universitari i Politècnic La Fe

Valencia, Spain

sanz_jai@gva.es

No potential conflict of interest relevant to this letter was reported.

1. Sanz J, Wagner JE, Sanz MA, et al. Myeloablative cord blood transplantation in adults with acute leukemia: comparison of two different transplant platforms. Biol Blood Marrow Transplant 2013;19:1725-30.

2. Atsuta Y, Kanda J, Takanashi M, et al. Different effects of HLA disparity on transplant outcomes after single-unit cord blood transplantation between pediatric and adult patients with leukemia. Haematologica 2013;98:814-22.

DOI: 10.1056/NEJMc1414419

THE AUTHORS REPLY: The observation that greater HLA mismatch is associated with better diseasefree survival after cord-blood transplantation is clearly provocative and merits additional evaluation. The question posed by Nusbaum regarding further delineation of the mechanism or mechanisms involved in this association - specifically, separating out the effect of matching between two units versus donor-recipient matching - is important but unfortunately was beyond the scope of our study. The complexity of double-unit cord-blood transplantation, in which we need to consider the effect of HLA matching in six directions rather than two in addition to understanding the effect of the HLA match of the predominating unit responsible for hematopoiesis in the long term, demands substantially larger sample sizes within each subgroup for a meaningful analysis.

In response to the comments by Sanz and colleagues: we too are particularly interested in segregating out the effect of the engrafting from the nonengrafting unit in recipients of a doubleunit cord-blood transplant. However, this is also a complex analysis that was beyond the scope of our study. It is possible that the immunologic graft-versus-graft response that is known to occur between the first and second cord-blood units might be responsible for some of the outcomes that we reported. For example, the higher incidence of acute GVHD after double-unit cordblood transplantation might be related to an "in vivo mixed lymphocyte response" between the two units, which in turn could have deleterious effects such as delayed platelet recovery ${ }^{1}$ but also beneficial effects such as a lower relapse rate. ${ }^{2}$ Alternatively, it is possible that the "tolerability" of greater HLA mismatch in patients receiving a cord-blood transplant unveils a greater graftversus-leukemia effect without the expected concomitant increase in transplantation-related mortality. In fact, the beneficial effect of HLA mismatch on relapse and survival after cordblood transplantation has already been reported. ${ }^{3}$ Although these possibilities are intriguing, they are only hypotheses at this time. If these observations hold in future studies, perhaps researchers who analyze databases with larger sample sizes can begin to explore the driving factors responsible for this apparent beneficial effect of HLA mismatch after double-unit cord-blood transplantation.

John E. Wagner, Jr., M.D.

University of Minnesota Medical School

Minneapolis, MN

wagne002@umn.edu

Mary Eapen, M.B., B.S.

Medical College of Wisconsin

Milwaukee, WI

Joanne Kurtzberg, M.D.

Duke University

Durham, NC

Since publication of their article, the authors report no further potential conflict of interest.

1. Ramírez P, Brunstein CG, Miller B, Defor T, Weisdorf D. Delayed platelet recovery after allogeneic transplantation: a predictor of increased treatment-related mortality and poorer survival. Bone Marrow Transplant 2011;46:981-6.

2. Verneris MR, Brunstein CG, Barker J, et al. Relapse risk after umbilical cord blood transplantation: enhanced graft-versusleukemia effect in recipients of 2 units. Blood 2009;114:4293-9. 3. Sanz J, Jaramillo FJ, Planelles D, et al. Impact on outcomes of human leukocyte antigen matching by allele-level typing in adults with acute myeloid leukemia undergoing umbilical cord blood transplantation. Biol Blood Marrow Transplant 2014;20: 106-10.

DOI: $10.1056 / N E J M c 1414419$

\section{Cardiovascular Risk and Events and Country Income Stratum}

TO THE EDITOR: Yusuf et al. (Aug. 28 issue) ${ }^{1}$ found that although the INTERHEART Risk Score classifies residents of high-income coun- tries as being at greater cardiovascular risk than residents of low- and middle-income countries, the rates of major cardiovascular events and 
death are substantially higher in low-income countries. This discrepancy may be explained by the omission of socioeconomic factors from the INTERHEART Risk Score. If these factors had been considered, a greater risk would probably have been predicted among the residents of less wealthy countries than was determined by the investigators, and consequently the inconsistency between the predicted and observed health outcomes would have been less than was observed.

The Moli-sani study has shown that healthful behaviors, such as adherence to a Mediterranean diet, are strongly linked to material resources, even in a high-income country such as Italy. ${ }^{2}$ Even small income differences produce a shaped gradient in modifiable risk factors, with more disadvantaged persons having not only more risk factors but also fewer protective factors than those with higher incomes. ${ }^{3}$ The work by Yusuf et al. is a further confirmation of the need to include socioeconomic factors in any approach aimed at predicting health risk, especially at a time of economic crisis when health has definite economic determinants. ${ }^{3}$

Marialaura Bonaccio, M.A.

Augusto Di Castelnuovo, Ph.D.

Licia lacoviello, M.D., Ph.D.

IRCCS Istituto Neurologico Mediterraneo Neuromed

Pozzilli, Italy

licia.iacoviello@neuromed.it

No potential conflict of interest relevant to this letter was reported.

1. Yusuf S, Rangarajan S, Teo K, et al. Cardiovascular risk and events in 17 low-, middle-, and high-income countries. N Engl J Med 2014;371:818-27.

2. Bonaccio M, Di Castelnuovo A, Bonanni A, et al. Decline of the Mediterranean diet at a time of economic crisis: results from the Moli-sani study. Nutr Metab Cardiovasc Dis 2014;24:853-60.

3. Bonaccio M, Bonanni AE, Di Castelnuovo A, et al. Low income is associated with poor adherence to a Mediterranean diet and a higher prevalence of obesity: cross-sectional results from the Moli-sani study. BMJ Open 2012;2(6):pii:e001685.

DOI: 10.1056/NEJMc1412846

TO THE EDITOR: Yusuf et al. conclude that the higher rates of cardiovascular disease observed in low- and middle-income countries, as compared with high-income countries, are unexplained by the prevalence of traditional cardiovascular risk factors, such as hypertension and diabetes. Unfortunately, the study did not consider two nontraditional factors that are important in poorer countries: household and ambient air pollution. Household air pollution from the use of solid fuels (biomass or coal) for cooking is ubiquitous among the world's poorest 3 billion people, ${ }^{1}$ and ambient air pollution is most severe in middle-income countries such as India and China. $^{2}$ The Global Burden of Disease study showed that in India approximately $35 \%$ of all cases of ischemic heart disease were due to household air pollution and nearly $30 \%$ were due to ambient air pollution, as compared, for example, with $29 \%$ of cases that were due to tobacco smoking. ${ }^{3}$ Evidence for the relationship between household air pollution and cardiovascular disease, unlike that for other risk factors, including ambient air pollution, does not currently derive from studies of disease but from studies of intermediate effect ${ }^{4}$ and directly linking evidence across four major types of combustion air pollution: smoking, household air pollution, secondhand tobacco smoke, and ambient air pollution. ${ }^{5}$

Kirk R. Smith, Ph.D., M.P.H.

John R. Balmes, M.D

University of California, Berkeley

Berkeley, CA

krksmith@berkeley.edu

Michael J. Guarnieri, M.D., M.P.H

University of California, San Francisco

San Francisco, CA

No potential conflict of interest relevant to this letter was reported.

1. Bonjour S, Adair-Rohani H, Wolf J, et al. Solid fuel use for household cooking: country and regional estimates for 19802010. Environ Health Perspect 2013;121:784-90.

2. Brauer M, Amann M, Burnett RT, et al. Exposure assessment for estimation of the global burden of disease attributable to outdoor air pollution. Environ Sci Technol 2012;46:652-60.

3. Lim SS, Vos T, Flaxman AD, et al. A comparative risk assessment of burden of disease and injury attributable to 67 risk factors and risk factor clusters in 21 regions, 1990-2010: a systematic analysis for the Global Burden of Disease Study 2010. Lancet 2012;380:2224-60.

4. McCracken JP, Wellenius GA, Bloomfield GS, et al. Household air pollution from solid fuel use: evidence for links to CVD. Global Heart 2012;7:223-34.

5. Burnett RT, Pope CA III, Ezzati M, et al. An integrated risk function for estimating the global burden of disease attributable to ambient fine particulate matter exposure. Environ Health Perspect 2014;122:397-403. [Erratum, Econ Health Perspect 2014; 122:A235.]

DOI: 10.1056/NEJMc1412846

TO THE EDITOR: Yusuf et al. found a striking inverse relationship in low-income countries between the lowest INTERHEART Risk Score (a composite score of traditional risk factors without the use of laboratory testing) and the highest rates of death from cardiovascular causes and major cardiovascular events. Their findings 
suggest a considerable, unattributed risk of cardiovascular disease in these resource-limited settings.

Atherosclerosis is a chronic inflammatory process that begins in early life, ${ }^{1}$ and several stimuli initiate and maintain the inflammatory state. Infectious diseases have long been implicated as potential contributors to the development of atherosclerosis and to plaque instability and rupture. ${ }^{2}$ Infections occur earlier in life and with greater frequency and severity in resourcelimited settings than they do in industrialized countries. ${ }^{3,4}$ The greater burden of infectious disease may contribute to the higher mortality from cardiovascular disease and more severe cardiovascular disease in income-poor settings than in asset-rich settings, despite the lower prevalence of traditional cardiovascular risk factors. Comparisons of country-specific rates of infection-related mortality and hospitalization, especially in childhood, should be feasible and may be informative regarding potentially modifiable but underappreciated risk factors for cardiovascular disease later in life.

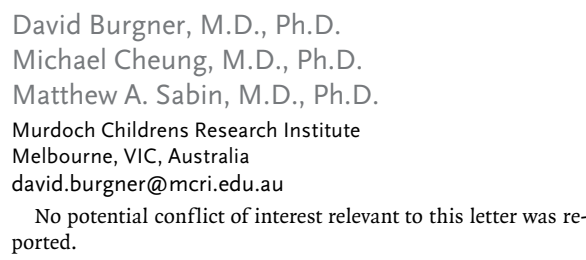

1. Libby P. Inflammation in atherosclerosis. Arterioscler Thromb Vasc Biol 2012;32:2045-51.

2. Rosenfeld ME, Campbell LA. Pathogens and atherosclerosis: update on the potential contribution of multiple infectious organisms to the pathogenesis of atherosclerosis. Thromb Haemost 2011;106:858-67.

3. Lozano R, Naghavi M, Foreman K, et al. Global and regional mortality from 235 causes of death for 20 age groups in 1990 and 2010: a systematic analysis for the Global Burden of Disease Study 2010. Lancet 2012;380:2095-128.

4. Vos T, Flaxman AD, Naghavi M, et al. Years lived with disability (YLDs) for 1160 sequelae of 289 diseases and injuries 1990-2010: a systematic analysis for the Global Burden of Disease Study 2010. Lancet 2012;380:2163-96.

DOI: $10.1056 / N E J M c 1412846$

THE AUTHOR REPLIES: The investigators in our study agree that socioeconomic factors are important. We are validating approaches that can be used across countries at differing economic levels, and we intend to apply them to the study population. In future analyses, we will be relating levels of outdoor and indoor air pollution to cardiovascular disease and also infections in adulthood (but not in childhood, because these data were not collected) to incident cardiovascular diseases.

Salim Yusuf, M.B., B.S., D.Phil.

Population Health Research Institute Hamilton, ON, Canada

Since publication of his article, the author reports no further potential conflict of interest.

\section{FOLFOXIRI and Bevacizumab for Metastatic Colorectal Cancer}

TO THE EDITOR: In their article on the Triplet plus Bevacizumab (TRIBE) study, Loupakis et al. (Oct. 23 issue) ${ }^{1}$ conclude that chemotherapy with fluorouracil, leucovorin, oxaliplatin, and irinotecan (FOLFOXIRI) plus bevacizumab improved the outcome of patients with metastatic colorectal cancer, as compared with a control group receiving fluorouracil, leucovorin, and irinotecan (FOLFIRI) plus bevacizumab. However, I question the basis for this conclusion.

First, although the median duration of progression-free survival was 12.1 months in the FOLFOXIRI group, as compared with 9.7 months in the control group, there was no significant improvement in overall survival with FOLFOXIRI. Second, FOLFOXIRI caused significantly more grade 3 or 4 events of neurotoxicity, diarrhea, neutropenia, and stomatitis, which are serious side effects. Third, even though tumors with nonmutated KRAS occurred in $37.3 \%$ of patients in the FOLFOXIRI group and $38.7 \%$ of those in the control group, subsequent treatment with an anti-epidermal growth factor receptor antibody (anti-EGFR) was performed in only $33 \%$ of patients in the FOLFOXIRI group and 29\% of those in the control group, and the KRAS mutation status is not indicated for any of these patients. In addition, anti-EGFR was administered as second-line treatment in $31 \%$ of the patients in the FOLFOXIRI group, as compared with only $15 \%$ of the patients in the control group.

This study commenced around the same time 
The purpose of this study was dual:

1. to assess whether adherence to the Mediterranean diet can still convey health advantages across different population groups and

2. to examine the socioeconomic and cultural determinants of adherence to this eating behavior. Additionally, the thesis has addressed the likely accounting of the economic crisis for the sudden decline of the adherence to Mediterranean diet.

\section{A number of crucial issues have been addressed:}

\section{Health advantages from a diet that underwent nutritional changes over the last decades}

The Mediterranean diet of the XXI century appears to confirm its beneficial effects for several different health outcomes.

Health-related quality of life, a valid predictor of mortality, has been proven to be strongly related to the Mediterranean eating behavior and the effect was partially mediated by the high antioxidant and fiber content that may exert an antiinflammatory action thus reducing the risk of disease (Chapter 3). Both in observational $(1,2)$ and intervention studies (3) adherence to a traditional Mediterranean diet has been reportedly associated with lower concentrations of inflammatory biomarkers such as C-reactive protein, fibrinogen, homocysteine or white blood cell count.

Such observations do offer further support to the inflammation-lowering theory which could account for the positive health benefits of the Mediterranean diet against the risk of major chronic diseases (4).

This hypothesis seems to be further supported by findings from Chapter 4 examining the relationship of a Mediterranean-like diet and two recognized markers of lowgrade inflammation. More in detail, we found that both white blood cell and platelet counts are favorably reduced by a Mediterranean-type diet. Basically, in a large population sample of apparently healthy subjects without any hematological disease, the adherence to a Mediterranean eating behavior reduces the number of both white blood cell and platelet counts, thus suggesting a prominent role of this dietary pattern in modulating the degree of subclinical inflammation.

Additionally and for the very first time, this chapter (4) has addressed dietary behaviors as a likely determinant of the number of platelets. 


\section{Favorable health outcomes within high risk populations}

Data presented in Chapter 5 addressed the issue whether Mediterranean diet has the same effect across population groups at different health risk. When the adherence to this pattern was proven to reduce mortality in the popular Greek EPIC cohort, the sample tested was free from major chronic diseases. The score elaborated by Antonia Trichopoulou and co-workers was found to be predictive of death within a sample of apparently healthy individuals. Later on, evidence emerged also from population groups at high risk for chronic disease (5). Studies on the elderly or subjects with prevalent chronic conditions have demonstrated that a Mediterranean behavior is effective also for high risk individuals. However, evidence on diabetes is scarce. A Mediterranean lifestyle is able to reduce the risk of the illness, but so far few studies have investigated a likely association between adherence to the Mediterranean diet and mortality in patients with diabetes (6). The work here discussed dealt with this issue and provided original knowledge on this not well explored field. The findings showed that people diagnosed with the illness at time of enrollment did benefit from having a Mediterranean diet by a significant lowering of the risk of dying of overall or cardiovascular causes.

Additionally, this effect was mainly accounted for by the moderate alcohol intake that has always played a tremendous role within the dietary groups of the Mediterranean pyramid (7).

All these works do support what argued in the viewpoint by Jeremiah Stamler (reference 2 from introduction); despite the unquestioned nutritional and behavioral changes, the traditional way of eating typical of the Mediterranean basin still has a say in the health business.

\section{Adherence to Mediterranean diet is socially patterned}

The socioeconomic gradient in adherence to the Mediterranean diet was presented in Chapter $\mathbf{6}$ as it has been recognized within the MOLI-SANI cohort.

The MOLI-SANI study is one of the largest population studies ever conducted in Europe that collected information and blood samples of about 25,000 citizens living in the Molise region, an area placed between the Northern and the Southern regions of the Country (8). This large amount of data, obtained between 2005 and 2010, allowed us to undertake targeted investigations both in genetics and environmental fields. Among the wide panel of information available, the MOLI-SANI study has a detailed section regarding socioeconomic status, both present and during childhood, including questions on household income. Such pieces of information are extremely useful in evaluating the role of material circumstances in accounting for several health outcomes, in addition to other more common risk factors. Regarding income, it is 
noteworthy to stress the fact that people are often reluctant to providesuch an information that is usually believed to be a quite sensitive issue and the risk of underreporting cannot be entirely excluded. Although the non-respondent category is well represented also in our cohort, the MOLI-SANI study has collected such data from family groups thus allowing a kind of control of the information provided by household members.

Findings discussed in Chapter $\mathbf{6}$ show that, as well as the whole Country, this Southern area of Italy is not characterized by huge socioeconomic gaps, at least not comparable to those reported in other European or North American countries. We found that adherence to the Mediterranean behavior is strictly linked to material resources independently of cultural circumstances, such as educational level. Indeed, greater income was associated with higher adherence to the Mediterranean diet and the association persisted in different education groups. The data are of interest since the differences we observed in the adherence to MD according to income indicate that also in an environment homogeneous both for genetics and lifestyles, income and education can still play a role in influencing dietary choices. Furthermore, the diet quality showed a continued improvement across a relatively small range of economic strata. Our 'poorest' are represented by people earning less than 10,000 Euro/net per year, while the 'richest' group is made of subjects with more than 40,000 Euros/year. Such differences among income classes are quite restrained and indicate a prominent role of material circumstances in determining the pursuing of healthy behaviors.

\section{Cultural determinants likely accounting for the Mediterranean diet gradient} Nutrition knowledge and mass media exposure are associated with greater adherence to the Mediterranean diet independently of other cultural circumstances (educational level) or material resources (Chapters 7 and 8 ).

Knowledge on health-related topics has become of great interest basically because of the direct relationship between diet quality, social and cultural factors and beliefs which are considered to be important factors explaining variations in food choices (9). Nutrition knowledge has also been shown to be a mediator between socioeconomic position and diet quality (10). Results presented in Chapter 8 indicate that dietary behaviors are dramatically linked to the amount of information on specific health-related issues. In addition to behaviors, nutrition knowledge is strictly associated also with lower rates of obesity. The pivotal importance of information is confirmed by the results presented in Chapter 7 aiming at estimating the association between the Mediterranean diet and the exposure to mass media. Similarly to what observed for nutritional knowledge, greater information is associated with increased 
conformity to the Mediterranean eating model. However, in the latter study, we did not actually evaluate the quality but only the amount of information that was obtained by detailed questions on how much time subjects spent in watching TV, surfing the web or reading (i.e. newspapers, magazines).

Findings show that both nutrition knowledge and exposure to mass media represent two main behavior-conditioning agents and their understanding is likely to be crucial to define new and more effective public health policies for the prevention of major chronic disease.

\section{The Italian Population is shifting from the traditional Mediterranean diet}

The Mediterranean behavior has started to lose its proselytizers time after time and the hugest losses were experienced in the Mediterranean countries.

Analyses undertaken within the whole period of the recruitment of the MOLI-SANI study clearly showed that adherence to the Mediterranean pattern dramatically changed over time (Chapter 9).

In respect to the years 2005-2006, a dramatic drop was recorded in 2007, the period when the global economic crisis became manifested, with prevalence of high adherence falling from over 30 percent to 18 percent in the whole population. This evidence led to search for the possible causes of this tough shifting. There were reasons to believe that, in addition to the cultural and social development, the changes affecting the material resources could likely account for the observed decline.

The Mediterranean diet fell around 2007 and the economic crisis became manifested at the same time. In order to test the hypothesis that the two phenomena could not be a result of a mere coincidence, the work here presented evaluated to what extent these dietary changes could be ascribed to socioeconomic factors.

The study (Chapter 9) postulated the economic downturn could play a role in the sudden decline of the number of people following a Mediterranean diet; the argument was supported by examining the contribution of different socioeconomic indicators to the adherence to this dietary pattern over a six year period. The dietary habits of people were grouped in two different timespans, before (2005-2006) and after the economic crisis started (2007-2010).

During 2005-2006 major socioeconomic indicators were not associated with having a Mediterranean behavior, which was conversely linked to age, sex, higher education and living in urban areas.

At variance, in 2007-2010 socioeconomic determinants began to play a major role in explaining the positive association with the Mediterranean diet. Household wealth, education, living in small villages and profession became largely associated with greater adherence to MD. 
We argued that the growing importance of socioeconomic indicators in determining dietary habits could find a likely explanation either in the rising cost of typical Mediterranean food items or in the higher rates of unemployment and reduction in terms of purchasing power of wages.

\section{The Mediterranean diet is no longer the diet of the poorest}

The findings presented in Chapters $\mathbf{6}$ and $\mathbf{9}$ are somehow contrasting with the traditional idea of the Mediterranean diet. For a long time, this eating behavior has been seen as the diet of the poorest population groups but nowadays is directly influenced by socioeconomic and cultural factors in line with what has been observed in non-Mediterranean settings (11). Healthy behaviors, including healthful diets, are definitely a prerogative of wealthy groups.

\section{The economic crisis as a major threat for the Mediterranean tradition}

On the basis of the findings presented in Chapter 9, there is reason to believe that in a period of economic crisis the burden of socioeconomic factors might rapidly accrue to wider social gaps across the population. Early investigations have tried to predict possible health outcomes deriving from the economic downturn by analyzing what already happened during previous economic crises. Regarding diet, viewpoints are contrasting. On one side, it has been argued that reduced material resources (i.e. income) would encourage people to spend more time in cooking at home with notable gains in terms of diet quality (12); on the other hand, it is likely that the increasing cost of the Mediterranean food would drive population, in particular the less advantaged which invest less in food, to look for cheapest and nutrient-poor foods (13).

If the latter is true, we shall expect an increase in inequities across socioeconomic groups and consequently in future health outcomes that are likely to become even more socially patterned. The important role played by socioeconomics has been largely addressed but few attempts have been made to include it as a major risk factor beyond the traditional ones. As an example, a recent paper reported that although the Interheart risk score poses high-income countries at greater cardiovascular risk, the rates of major events and death are substantially higher in low-income countries (14). To explain this apparent discrepancy, the authors did not include any socioeconomic evaluation of the individuals living in different countries and economical settings. In a letter to the Editor of the New England Journal of Medicine (Chapter 10) we argued that if these factors had been considered we would likely have assisted to a greater predicted risk for the less wealthy and consequent reduced inconsistency between prediction and health outcomes. Referring to data reported in this thesis 
(Chapters 6 and 9) we recalled that even small income differences produce a shaped gradient in modifiable risk factors with the more disadvantaged having not only more risk factors but also less protective factors. The work by Yusuf is a further confirmation of the need to include socioeconomic factors in any approach aimed to predict health risk especially in population settings with huge social gaps.

As a general conclusion, the likely impact of the economic crisis on health should be a crucial issue when defining new strategies for the prevention of major chronic disease at a time when also diet and health in general have definitely confirmed their economic nature.

\section{WHAT THIS THESIS ADDS}

Data addressing the reported decline of the Mediterranean diet in Italy are scarce. The papers included in this thesis do add knowledge on this pivotal topic that is expecting to have a direct impact on public health policies. Obesity rates have dramatically increased and the burden of diet-related chronic conditions (i.e. diabetes) is no longer affordable. The Mediterranean diet has always been linked to improved general health, thus the shifting from it is likely to further worsen the healthcare sector.

Additionally and apparently for the first time, the findings here discussed have suggested a clear relationship between the Mediterranean behavior and the occurrence of the economic crisis by estimating the contribution of the most widely used socioeconomic indicators in explaining the association.So far, possible unfavorable health outcomes deriving from the economic downturn haveonly been predicted on the basis of what happened during previous recessions. Attention has mainly been paid to immediate rises in suicides and falls in road traffic deaths or HIV outbreaks, considered as products of state retrenchment (12). Actually, unintended modifications of lifestyles and following health consequences were not extensively discussed. This thesis draws a particular attention to the likely negative outcomes deriving from economic constraints. Poor income has been proven to be associated with higher prevalence of obesity and lower adherence to the Mediterranean diet. If in future years income should become a major determinant of the adherence to Mediterranean diet we should likely expect an increase in the rates of obesity and a general worsening of health status. 
1. Chrysohoou C, Panagiotakos DB, Pitsavos C, Das UN, Stefanadis C. Adherence to the Mediterranean diet attenuates inflammation and coagulation process in healthy adults: The ATTICA Study. J Am Coll Cardiol 2004: 44;152-158.

2. Centritto F, Iacoviello L, di Giuseppe R, De Curtis A, Costanzo S, Zito F, Grioni S, Sieri S, Donati MB, de Gaetano G, Di Castelnuovo A; Moli-sani Investigators. Dietary patterns, cardiovascular risk factors and C-reactive protein in a healthy Italian population. Nutr Metab Cardiovasc Dis. 2009;19:697-706.

3. Ambring A, Johansson M, Axelsen M, Gan L, Strandvik B, Friberg P. Mediterranean-inspired diet lowers the ratio of serum phospholipid n-6 to n-3 fatty acids, the number of leukocytes and platelets, and vascular endothelial growth factor in healthy subjects. Am J Clin Nutr. 2006;83:575-81.

4. Giacosa A, Barale R, Bavaresco L, et al. Cancer prevention in Europe: the Mediterranean diet as a protective choice. Eur J Cancer Prev. 2013;22:90-5.

5. Trichopoulou A, Bamia C, Trichopoulos D. Mediterranean diet and survival among patients with coronary heart disease in Greece. Arch Intern Med. 2005 25;165:929-35.

6. Iimuro S, Yoshimura Y, Umegaki H, Sakurai T, Araki A, Ohashi Y, Iijima K, Ito H; Japanese Elderly Diabetes Intervention Trial Study Group. Dietary pattern and mortality in Japanese elderly patients with type 2 diabetes mellitus: does a vegetable- and fish-rich diet improve mortality? An explanatory study. Geriatr Gerontol Int. 2012;12 Suppl 1:59-67.

7. Trichopoulou A, Bamia C, Trichopoulos D. Anatomy of health effects of Mediterranean diet: Greek EPIC prospective cohort study. BMJ. 2009;338:b2337.

8. Iacoviello L, Bonanni A, Costanzo S, De Curtis A, Di Castelnuovo A, Olivieri M, Zito F, Donati MB, de Gaetano G and Iacoviello L; MOLI-SANI project investigators. The MOLI-SANI Project, a randomized, prospective cohort study in the Molise region in Italy; design, rationale and objectives. Italian J Public Health. 2007;4:110-118.

9. Sharma SV, Gernand AD, Day RS. Nutrition knowledge predicts eating behavior of all food groups except fruits and vegetables among adults in the Paso del Norte region. Qué Sabrosa Vida. Journal of Nutrition Education and Behavior. 2008; 40: 361-368.

10.McLeod ER, Campbell KJ, Hesketh KD. Nutrition knowledge. A mediator between socioeconomic position and diet quality in Australian first time mothers. Journal of the American Dietetic Association. 2011;111: 696-704. 
11. Darmon N, Drewnowski A. Does social class predict diet quality? Am J Clin Nutr 2008;87:1107-17.

12.Karanikolos M, Mladovsky P, Cylus J, Thomson S, Basu S, Stuckler D, Mackenbach JP, McKee M. Financial crisis, austerity, and health in Europe. Lancet. 2013;381:1323-3.

13. Monsivais P, Aggarwal A, Drewnowski A. Are socio-economic disparities in diet quality explained by diet cost? J Epidemiol Community Health. 2012;66:530-5.

14. Yusuf S, Rangarajan S, Teo K et al. Cardiovascular risk and events in 17 lowmiddle-, and high-income countries. N Engl J Med. 2014;371:818-27. 
The health care costs attributable to diseases and conditions related to nutrition are large with about 1.7 million of deaths worldwide attributable to nutrient-poor diets, such as those low in fruit and vegetables (1). The global economic burden of cardiovascular disease and cancer has been estimated in 290 and 863 billion dollars, respectively, and is expected to rise to 458 billion dollars and 1.04 trillion dollars by 2030 , respectively (2). On the other side, about $44 \%$ of the diabetes burden, $23 \%$ of the ischaemic heart disease burden and between $7 \%$ and $41 \%$ of certain cancer burdens are attributable to overweight and obesity, two conditions strongly linked to unhealthy lifestyles (3).

A Mediterranean dietary pattern, that encourages large intakes of fruits and vegetables, low consumption of meat and dairy products, olive oil as main fat source and moderate alcohol intake during meals, has proven to be cost/effective for a wide series of diseases and conditions, including secondary cardiovascular prevention $(4,5)$.

As reported by several investigations, a healthy and balanced diet can reduce the incidence of major chronic diseases and mortality. For people at high cardiovascular risk, a Mediterranean diet enhanced with olive oil or nuts turned out to be effective in reducing the cardiovascular risk with significant health advantages for both individuals and healthcare system (6). Accordingly, a Mediterranean dietary pattern has proven to reduce the incidence of diabetes by about 20 percent and the risk of both overall and cardiovascular death in those suffering from the illness by around 30 percent (7). Diabetes affects approximately 400 million people worldwide (8) and is estimated to become the 7th leading cause of death in 2030 (9) with enormous social and healthcare costs estimated in 745 billion dollars in 2030 (2).As dramatically stressed by the Organization for Economic Cooperation and Development, "preventing and treating diabetes and its complications costs about $€ 90$ billion annually in Europe alone. With health budgets already under great pressure and national budgets severely strained, for the sake of our health and the health of our economies we must find ways to prevent and manage diabetes in a costeffective manner" (10). The reduction of the burden of diabetes by promotion of healthy lifestyles, including diet, appears to be the best action to get huge gains in terms of savings (8).

More recently, a Mediterranean-type diet has been associated with reduced risk of depression (11) that represents the second leading cause of disability worldwide, thus becoming a public health priority, leading scientists to call for actions aimed at 
implementing cost-effective interventions to reduce its burden (12).

Promotion of healthy lifestyles still represents a highly cost-effective tool for prevention of major chronic diseases and the Mediterranean diet is not the exception. Its advancement is of primary importance especially at a time of economic crisis when policies for containing health care costs may risk to lose sight of what is unconditionally needed for people's health.

Interestingly, the Mediterranean diet has been recently proposed by UNESCO as a global worthy model of eating (13).Among the reasons provided, the Mediterranean diet has been recognized the capacity of conferring a sense of belonging and sharing and of raising awareness of the significance of healthy and sustainable food related practices in other parts of the world (13).

The Mediterranean diet does have a planet-saving dimension as well; with its low intakes of meat or meat products this eating model has also been seen as a helpful tool to reduce the carbon dioxide emissions linked to giant livestock farms with consequent benefits for the environment. Additionally, its promotion of local food products may represent an advantageous opportunity for the economic revitalization of small production companies.

Rather than a simple way of eating, the Mediterranean diet appears to be an inclusive way of living the very next future both at individual and global level.

\section{CHAPTER 12 REFERENCES}

1. http://www.who.int/dietphysicalactivity/fruit/en/index2.html. Accessed September 2014

2. The global economic burden of non-communicable disease, World economic forum and Harvard school of public health, September 2011.

3. http://www.who.int/mediacentre/factsheets/fs311/en/. Accessed September 2014

4. Dalziel K, Segal L, de Lorgeril M. A Mediterranean diet is cost-effective in patients with previous myocardial infarction. J Nutr. 2006;136:1879-85.

5. de Lorgeril $\mathrm{M}$ et al. Mediterranean dietary pattern in a randomized trial: prolonged survival and possible reduced cancer rate. Arch Intern Med. 1998;158:1181-7.

6. Estruch $\mathrm{R}$ et al. Primary prevention of cardiovascular disease with a Mediterranean diet. N Engl J Med. 2013;368:1279-90.

7. Bonaccio $\mathrm{M}$ et al. Adherence to the traditional Mediterranean diet and mortality in subjects with diabetes. Prospective results from the MOLI-SANI study. Eur J Prev Cardiol. 2015 Feb 3. pii: 2047487315569409. [Epub ahead of print] 
8. Danaei $\mathrm{G}$ et al. National, regional, and global trends in fasting plasma glucose and diabetes prevalence since 1980: systematic analysis of health examination surveys and epidemiological studies with 370 country-years and 2.7 million participants. Lancet 2011; 378:31-40.

9. Global status report on noncommunicable diseases 2010. Geneva, World Health Organization, 2011.

10.http://www.oecd.org/eu/healththehighcostofdiabetes.htm. Accessed September 2014

11.Psaltopoulou $\mathrm{T}$ et al. Mediterranean diet, stroke, cognitive impairment, and depression: A meta-analysis. Ann Neurol. 2013;74:580-91.

12.Ferrari AJ et al. Burden of depressive disorders by country, sex, age, and year: findings from the global burden of disease study 2010. PLoS Med.;10:e1001547. 13.http://www.unesco.org/culture/ich/RL/00884 

Take-home messages may be summarized as follows:

1. The Mediterranean diet provides significant health advantages for different population groups; it may reduce low grade inflammation parameters such as platelet and white cell counts and decrease the risk of death for any cause in diabetic patients;

2. Conformity to this eating behavior dramatically fell down during the last few years, especially in Mediterranean countries;

3. Adherence to the Mediterranean diet is directly influenced by socioeconomic and cultural aspects;

4. A Mediterranean diet gradient is exhibited also in communities with poor socioeconomic gaps;

5. Socioeconomic determinants of adherence to the Mediterranean diet differed according to timespans with the increased role of material resources recorded after the economic crisis became manifested;

6. The global economic downturn is expected to further increase socioeconomic inequalities in health. 

A large body of evidence has been linking the traditional Mediterranean diet to reduced risk of major chronic diseases, such as cardiovascular and cerebrovascular disease, tumors and also neurodegenerative disease.

As largely described by the pioneering investigations on this topic, this dietary model is mainly characterized by abundance of fruit and vegetables, non-refined grains, cereals, nuts, legumes, fish, olive oil as main fat sources and moderate wine consumption preferably during meals.

However, the dietary behaviors of populations have been radically changing since the original definition of this eating pattern and many problematic nutritional aspects emerged. Basically, the foods we eat today are different from what the traditional Mediterranean populations used to have. This thesis addresses whether adherence to a Mediterranean-style diet is still available to convey health advantages both for apparently healthy subjects and for groups at high cardiovascular risk.

In addition, late evidence has been suggesting that Mediterranean societies are rapidly withdrawing from this eating pattern orienting their food choices toward products typical of the Western diet pattern. Among possible causes, the increasing cost of many key-foods of the Mediterranean diet is likely to have led people to give up this eating pattern in favor of less expensive products which allow to save money but are definitively unhealthy. Additionally, there is reason to believe that the economic downturn is going to produce unfavorable effects on the dietary lifestyle of people, especially among the less wealthy groups. Thus, the second part of this thesis focuses on the role of either material or cultural resources in determining the conformity to the Mediterranean diet and also analyzes their accounting for the dramatic decrease of the adherence recorded in the latest years. Consistently, the thesis discusses whether the economic crisis could be considered as one of the likely causes to explain the shifting from this healthy dietary pattern.

As far as the valorization of this thesis is concerned, several points have been mentioned including the cost effectiveness of the Mediterranean diet and its cultural and planet-saving dimension (as recently recognized by UNESCO). 



\section{Marialaura Bonaccio, MA}

\section{Personal details}

- Date of birth: January 22, 1981

- Place of birth: Campobasso, Molise, Italy

- Citizenship: Italian

- Civil status: single

- Address: 7 Porta Mancina 86100, Campobasso, Italy

- Telephone: +339 4995848 (mobile)

- Email: marialaura.bonaccio@moli-sani.org

\section{Education}

- 1994-1999: High School at Liceo Scientifico A. Romita, Campobasso, Italy.

- 1999-2004: MA in Philosophy, Faculty of Humanities and Philosophy, Università degli studi di Cassino e del Lazio Meridionale, Cassino (FR), Italy (the degree of Dottore in Filosofia is equal to a master degree obtained at a Dutch University).

- June-July 2013 - European Educational Programme in Epidemiology - 26th Residential Summer Course in Epidemiology (Summer 2013, Florence, Italy).

- 2012-2015: PhD student at CARIM School for Cardiovascular Diseases, Maastricht University, The Netherlands.

$\mathrm{PhD}$ thesis title: Health benefits and social determinants of the Mediterranean diet at a time of economic crisis: results from the Moli-sani study.

\section{Research and working experience}

- 2015-2016: Post-Doctoral Fellowship granted by the Fondazione Umberto Veronesi, Milano, Italy.

- 2013-2015: Research fellow in nutrition and social epidemiology at the Department of Epidemiology and Prevention, IRCCS Istituto Neurologico Mediterraneo NEUROMED, Pozzilli, Molise, Italy

- 2006-2012: Research fellow and science communicator at the Research Laboratories of the Catholic University of the Sacred Heart, Campobasso, Italy

\section{Science communication experience}

- Teaching science communication at the Istituto Neurologico Mediterraneo 
NEUROMED in Pozzilli.

- European Project FLORA 6th Framework - Communication manager 2005 - 2009.

- uropean Project ATHENA 7th Framework - Communication manager 2010 2013.

- Science journalist and author of many press articles on health and prevention for public dissemination.

\section{Publications}

- First author of 9 peer-reviewed publications and of 4 invited chapters.

- Co-author of over 10 peer-reviewed publications as an investigator of the Molisani study.

- $\mathrm{H}$-Index (calculated via Google scholar) $=10$.

- Co-author, together with Giovanni de Gaetano, of the book "La dieta mediterranea ai tempi della crisi”" (Il Pensiero Scientifico editore, Roma, 2012).

\section{Participation to scientific congresses as an invited speaker}

- October 2014, World Thrombosis Day, Lucera, Italy.

- May 2013, Workshop on the Mediterranean diet, Bari.

- April 2013, Europrevent, Rome.

- November 2011, XXXVII Corso di Aggiornamento "Alterazioni Congenite ed Acquisite della Coagulazione", Milano. 
1. Bonaccio M, Di Castelnuovo A, Costanzo S, Persichillo M, De Curtis A, Donati MB, de Gaetano G, Iacoviello L; on behalf of the MOLI-SANI study Investigators. Adherence to the traditional Mediterranean diet and mortality in subjects with diabetes. Prospective results from the MOLI-SANI study. Eur J Prev Cardiol. 2015 Feb 3. pii: 2047487315569409. [Epub ahead of print].

2. Bonaccio M, Di Castelnuovo A, Iacoviello L. Cardiovascular risk and events and country income stratum. N Engl J Med. 2015;372:288-9.

3. Bonaccio M, Di Castelnuovo A, Bonanni A, Costanzo S, De Lucia F, Persichillo M, Zito F, Donati MB, de Gaetano G and Iacoviello L on behalf of the Moli-sani project investigators. Decline of the Mediterranean diet at a time of economic crisis. Results from the Moli-sani study. Nutr Metab Cardiovasc Dis. 2014;24:85360.

4. Bonaccio M, Di Castelnuovo A, De Curtis A, Costanzo S, Persichillo M, Donati MB, Cerletti C, Iacoviello L, de Gaetano G. Adherence to the Mediterranean diet is associated with lower platelet and leukocyte counts: results from the Moli-sani study. Blood. 2014;123:3037-44.

5. Pounis G, Costanzo S, Persichillo M, de Curtis A, Sieri S, Vinceti M, Zito F, Di Castelnuovo AF, Donati MB, de Gaetano G, Iacoviello L; Moli-sani Project Investigators. Mushroom and dietary selenium intakes in relation to fasting glucose levels in a free-living Italian adult population: the Moli-sani Project. Diabetes Metab. 2014;40:34-42.

6. Bonanni AE, Bonaccio M, di Castelnuovo A, de Lucia F, Costanzo S, Persichillo M, Zito F, Donati MB, de Gaetano G, Iacoviello L. Food labels use is associated with higher adherence to mediterranean diet: results from the moli-sani study. Nutrients. 2013;5:4364-79.

7. Bonaccio M, Di Castelnuovo A, Bonanni A, Costanzo S, De Lucia F, Zito F, Pounis G, Donati MB, de Gaetano G, Iacoviello L. Adherence to a Mediterranean diet is associated with a better health-related quality of life: a possible role ofhigh dietary antioxidant content.BMJ Open. 2013 Aug 13;3(8).

8. Bonaccio M, Di Castelnuovo A, Costanzo S, De Lucia F, Olivieri M, Donati MB, de Gaetano G, Iacoviello L, Bonanni A. Nutrition knowledge is associated with higher adherence to Mediterranean diet and lower prevalence of obesity. Results from the Moli-sani study. Appetite. 2013;68:139-46.

9. Bonaccio M, Bonanni AE, Di Castelnuovo A, De Lucia F, Donati MB, de Gaetano 
$\mathrm{G}$ and Iacoviello L, on behalf of the Moli-sani Project Investigators. Low income is associated with poor adherence to a Mediterranean diet and a higher prevalence of obesity: cross-sectional results from the Moli-sani study. BMJ Open 2012;2:e001685. doi:10.1136/bmjopen-2012-001685

10. Bonaccio M, Di Castelnuovo A, Costanzo S, De Lucia F, Olivieri M, Donati MB, de Gaetano G, Iacoviello L, Bonanni A. Mass media information and adherence to Mediterranean diet: results from the Moli-sani study. Int J Public Health. 2012;57:589-97.

11. Bonaccio M, Iacoviello L, de Gaetano G, On Behalf Of The Moli-Sani Investigators. The Mediterranean diet: The reasons for a success. Thromb Res. 2012;129:401-4.

12.Rago L, Di Castelnuovo A, Assanelli D, Badilini F, Vaglio M, Gianfagna F, Salvetti M, Zito F, Alessandrini F, Donati MB, de Gaetano G, Iacoviello L, Twave axis deviation, metabolic syndrome and estimated cardiovascular risk In men and women of the MOLI-SANI Study. Atherosclerosis. 2013;226:412-8.

13. Arcari A, Magnacca S, Bracone F, Costanzo S, Persichillo M, Di Castelnuovo A, de Curtis A, Zito F, Schünemann HJ, Donati MB, de Gaetano G, Iacoviello L. Relation between pulmonary function and 10-year risk for cardiovascular disease among healthy men and women in Italy: the Moli-sani Project. Eur J Prev Cardiol. 2013;20:862-71.

14.Assanelli D, Rago L, Salvetti M, Di Castelnuovo A, Badilini F, Vaglio M, Zito F, Donati MB, de Gaetano G, Iacoviello L; on behalf of the Moli-sani Project Investigators. T-wave axis deviation, metabolic syndrome and cardiovascular risk: results from the MOLI-SANI study. J Electrocardiol. 2012;45:546-550.

15.Iacoviello L, Rago L, Costanzo S, Di Castelnuovo A, Zito F, Assanelli D, Badilini F, Donati MB, de Gaetano G; on behalf of the Moli-sani Project Investigators. The Moli-sani project: computerized ECG database in a population-based cohort study. J Electrocardiol. 2012;45:684-689.

16.di Giuseppe R, Arcari A, Serafini M, Di Castelnuovo A, Zito F, De Curtis A, Sieri S, Krogh V, Pellegrini N, Schünemann HJ, Donati MB, de Gaetano G, Iacoviello L; Moli-sani Project Investigators. Total dietary antioxidant capacity and lung function in an Italian population: a favorable role in premenopausal/never smoker women. Eur J Clin Nutr. 2012;66:61-8.

17. di Giuseppe R, Di Castelnuovo A, Melegari C, De Lucia F, Santimone I, Sciarretta A, Barisciano P, Persichillo M, De Curtis A, Zito F, Krogh V, Donati MB, de Gaetano G, Iacoviello L, on behalf of the Moli-sani Project Investigators. Typical breakfast food consumption and risk factors for cardiovascular disease in a large sample of Italian adults. Nutr Metab Cardiovasc Dis. 2012;22:347-54. 
18.Costanzo S, Gianfagna F, Persichillo M, De Lucia F, Verna A, Djidingar M,Magnacca S, Bracone F, Olivieri M, Donati MB,de Gaetano G, Iacoviello L. Pandemic and seasonal vaccine coverage and effectiveness during the 2009-2010 pandemic influenza in an Italian adult population. Int J Public Health. 2012;57:569-79.

19.Di Castelnuovo A, Costanzo S, Persichillo M, Olivieri M, de Curtis A, Zito F, Donati MB, de Gaetano G, Iacoviello L. Distribution of short and lifetime risks for cardiovascular disease in Italians. Eur J Prev Cardiol. 2012;19:723-30.

20. Santimone I, Di Castelnuovo AF, de Curtis A, Spinelli M, Cugino D, Gianfagna F, Zito F, Donati MB, Cerletti C, de Gaetano G, Iacoviello L. White blood cells count, sex and age are major determinants of platelet indices heterogeneity in an adult general population: results from the MOLI-SANI project. Haematologica. 2011;96:1180-8.

21.Centritto F, Iacoviello L, di Giuseppe R, De Curtis A, Costanzo S, Zito F, Grioni S, Sieri S, Donati MB, de Gaetano G, Di Castelnuovo A; Moli-sani Investigators. Dietary patterns, cardiovascular risk factors and C-reactive protein in a healthy Italian population. Nutr MetabCardiovasc Dis. 2009;19:697-706.

22.di Giuseppe R, Di Castelnuovo A, Centritto F, Zito F, De Curtis A, Costanzo S, Vohnout B, Sieri S, Krogh V, Donati MB, de Gaetano G, Iacoviello L.Regular consumption of dark chocolate is associated with low serum concentrations of Creactive protein in a healthy Italian population. J Nutr. 2008;138:1939-45.

23.Iacoviello L et al. The Moli-Sani Project, a randomized, prospective cohort study in the Molise region in Italy; design, rationale and objectives. Italian J Pub Health 2007; 4: 110-8.

\section{BOOK CHAPTERS}

1. Bonaccio M, Di Castelnuovo A, Bonanni A, de Gaetano G, Iacoviello L. Mediterranean diet and health in the Italian population. In: Martinez JR, ed. Avances en Alimentación, Nutrición y Dietética. In press

2. Bonaccio M, de Gaetano G, Iacoviello L. Effect of a Mediterranean Diet on Mental and Physical Quality of Life. In: Akhlaq A. Farooqui, Tahira Farooqui, eds. Diet and Exercise in Cognitive Function and Neurological Diseases. Wiley Blackwell, 2015: 39-45. 
3. Bonaccio M, Cerletti C, Iacoviello L, de Gaetano G. Mediterranean Diet and subclinical chronic inflammation: the MOLI-SANI Study. In: Jirillo E, Serafini M, eds. Proceedings of the VII Immunonutrition Workshop (Carovigno, Italy May 1-3, 2014). Endocr Metab Immune Disord Drug Targets. 2014 Oct 20. [Epub ahead of print].

4. Bonaccio M, Bonanni A, Donati MB, de Gaetano G, Iacoviello L. Information, Culture and Socioeconomics as Determinants of Adherence to Mediterranean Diet. In: Preedy VR, Watson RR, eds. The Mediterranean Diet An Evidence-Based Approach. Academic Press,2014: 589-597.

5. Pounis G, Bonaccio M, Tuohy K, Donati MB, de Gaetano G, Iacoviello L. Population Level Divergence from the Mediterranean Diet and the Risk of Cancer and Metabolic Disease. In: Tuohy K, Del Rio D, eds. Diet-Microbe Interactions in the Gut. Academic Press, 2014 


\section{CHAPTER 17. ACKNOWLEDGMENTS}

My gratitude goes to the following people who believed in me.

To Prof. Giovanni de Gaetano, who enthusiastically wanted a philosopher to join his group giving me the opportunity to fully understand that "there is only one knowledge". Now I do believe in that and thank him for his mentoring during all these years.

To Prof. Maria Benedetta Donati and Prof. Licia Iacoviello whose expertise, guidance, and encouragement actively supported me in my research career.

$\mathrm{My} \mathrm{PhD}$ experience is the return of their efforts and beliefs.

I wish to thank also Dr. Augusto Di Castelnuovo for introducing me to the intricacies of statistics, Americo Bonanni with whom I shared an enthusiastic experience of science communication, Chiara Cerletti and my colleagues and friends of the Department of Epidemiology and Prevention in Pozzilli whose hard work made possible mine.

This thesis has been conceived and developed in the framework of the Moli-sani study that is currently ongoing at the IRCCS Istituto Neurologico Mediterraneo NEUROMED in Pozzilli to which I'm sincerely grateful.

The recruitment phase was initiated and completed at the Catholic University in Campobasso.

I am grateful to Prof. Coen Hemker and Prof. Hugo ten Cate for accepting me as a $\mathrm{PhD}$ student.

My final thoughts go to my mother, my father and my sisters Valentina and Alessandra: somehow, the way they intend life has produced good things. 
Printed in February 2015

Tipolitografia Fotolampo srl

Campobasso - Italy 